\title{
ACTUATOR DISK THEORY FOR COMPRESSIBLE FLOW
}

\author{
A Thesis \\ presented to \\ the Faculty of California Polytechnic State University, \\ San Luis Obispo
}

\author{
In Partial Fulfillment \\ of the Requirements for the Degree \\ Master of Science in Mechanical Engineering
}

by

Htet Htet Nwe Oo

May 2017 
(C) 2017

Htet Htet Nwe Oo

ALL RIGHTS RESERVED 


\section{COMMITTEE MEMBERSHIP}

TITLE: Actuator Disk Theory for Compressible Flow

AUTHOR: $\quad$ Htet Htet Nwe Oo

DATE SUBMITTED: $\quad$ May 2017

COMMITTEE CHAIR: $\quad$ Russell Westphal, Ph.D.

Professor of Mechanical Engineering

COMMITTEE MEMBER: $\quad$ Christopher C. Pascual, Ph.D.

Professor of Mechanical Engineering

COMMITTEE MEMBER: Hans Mayer, Ph.D.

Lecturer of Mechanical Engineering 


\section{ABSTRACT \\ Actuator Disk Theory for Compressible Flow}

Htet Htet Nwe Oo

Because compressibility effects arise in real applications of propellers and turbines, the Actuator Disk Theory or Froude's Momentum Theory was established for compressible, subsonic flow using the three laws of conservation and isentropic thermodynamics. The compressible Actuator Disk Theory was established for the unducted (bare) and ducted cases in which the disk was treated as the only assembly within the flow stream in the bare case and enclosed by a duct having a constant cross-sectional area equal to the disk area in the ducted case. The primary motivation of the current thesis was to predict the ideal performance of a small ram-air turbine (microRAT), operating at high subsonic Mach numbers, that would power an autonomous Boundary Layer Data System during test flights. The compressible-flow governing equations were applied to a propeller and a turbine for both the bare and ducted cases. The solutions to the resulting system of coupled, non-linear, algebraic equations were obtained using an iterative approach. The results showed that the power extraction efficiency and the total drag coefficient of the bare turbine are slightly higher for compressible flow than for incompressible flow. As the free-stream Mach increases, the Betz limit of the compressible bare turbine slightly increases from the incompressible value of 0.593 and occurs at a velocity ratio between the far downstream and the free-stream that is lower than the incompressible value of 0.333 . From incompressible to a free-stream Mach number of 0.8 , the Betz limit increases by 0.021 while its corresponding velocity ratio decreases by 0.036 . The Betz limit and its corresponding velocity ratio for the ducted turbine are not affected by the free-stream Mach and are the same for both incompressible and compressible flow. The total drag coefficient of the ducted turbine is also the same regardless of the free-stream Mach number and the compressibility of the flow; but, the individual contributions of the turbine drag and the lip thrust to the total drag differs between compressible and incompressible flow and between varying free-stream Mach numbers. It was concluded that overall compressibility has little influence on the ideal performance of an actuator disk.

Keywords: Actuator Disk Theory, Froude's Momentum Theory, compressible flow, Betz limit 


\section{ACKNOWLEDGMENTS}

I would like to sincerely thank Dr. Russell Westphal for his undying support, his love and much appreciated enthusiasm for Fluid Mechanics, and his contiguous coffee-drinking. You are truly an inspiration and everything I aspire to be.

Thank you to Dr. Christopher C. Pascual and Dr. Hans Mayer for taking the time to review my thesis and for being a part of the committee.

I would like to thank my family, especially my loving parents, Ko Ko Zin and Laura Lynn, for

their endurance, strength, and sacrifice so that my brother, Kaung Zin Htoo, and I can prosper and live a life of freedom and opportunities.

Lastly, I would like to thank Northrop Grumman Corporation for their financial support of the Boundary Layer Data System (BLDS) and the current thesis. 


\section{TABLE OF CONTENTS}

Page

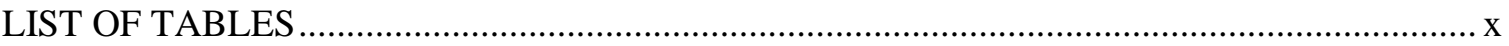

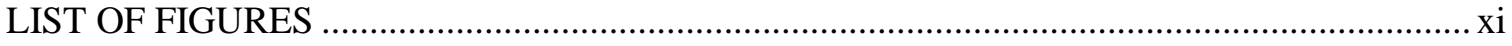

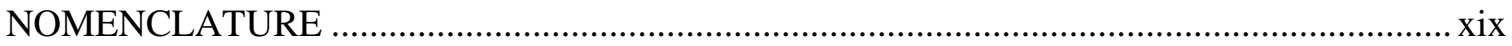

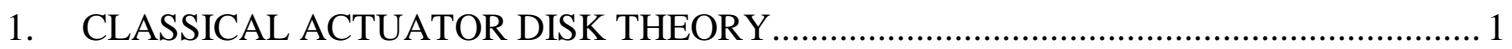

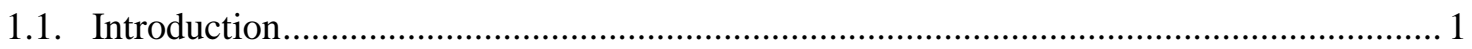

1.2. Classical Actuator Disk Theory - Governing Equations ............................................ 3

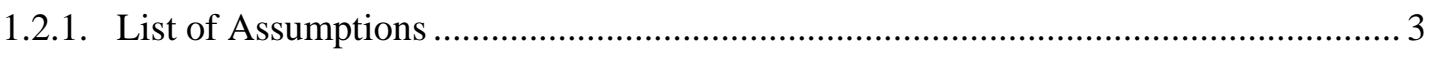

1.2.2. Incompressible, Bare Actuator Disk Theory ......................................................... 5

1.2.3. Incompressible, Ducted Actuator Disk Theory....................................................... 9

1.3. Applications of the Classical Actuator Disk Theory .................................................... 15

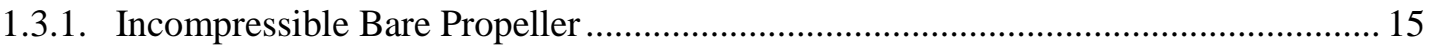

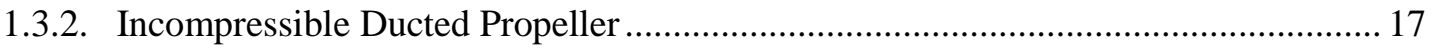

1.3.3. Results and Discussion - Incompressible Bare and Ducted Propeller....................... 18

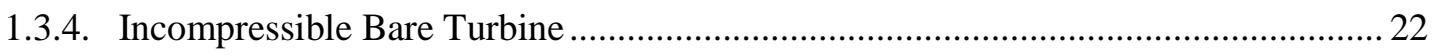

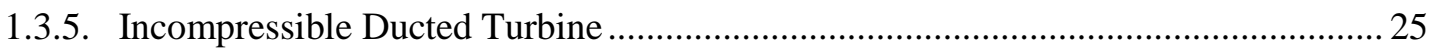

1.3.6. Results and Discussion - Incompressible Bare and Ducted Turbine........................ 26

1.4. Method of Solution to the Classical Actuator Disk Theory ............................................. 31

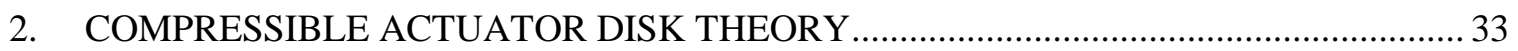

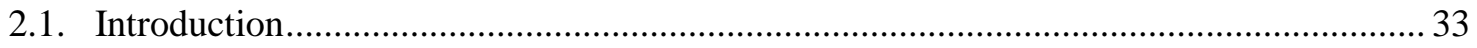

2.2. Compressible, Bare Actuator Disk Theory …........................................................... 35

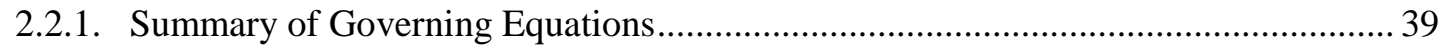




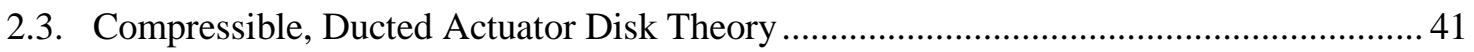

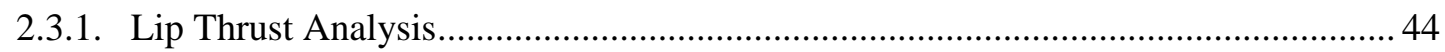

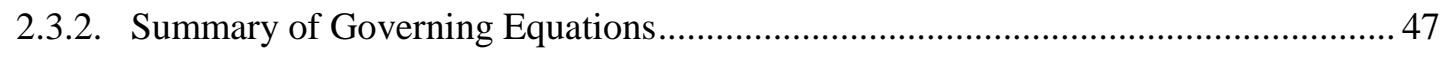

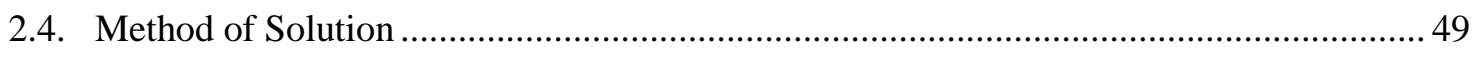

3. APPLICATIONS OF THE COMPRESSIBLE ACTUATOR DISK THEORY -

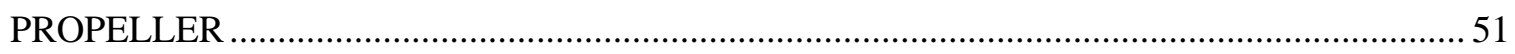

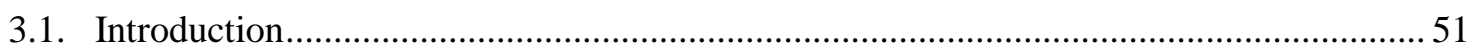

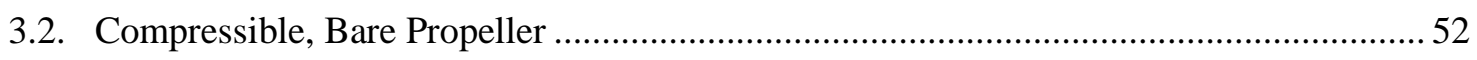

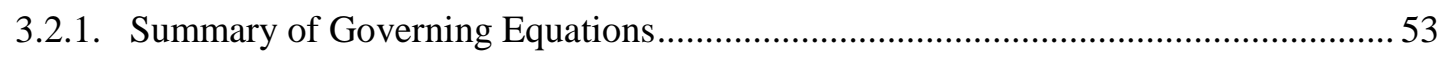

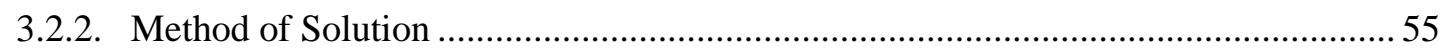

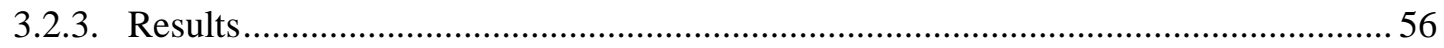

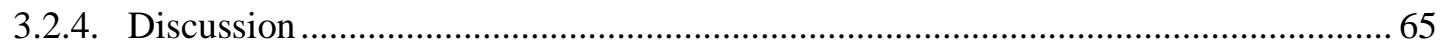

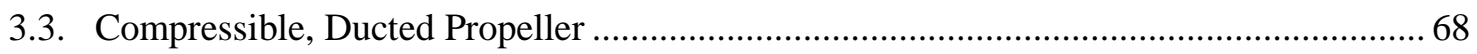

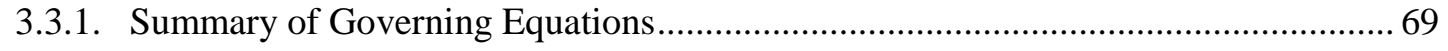

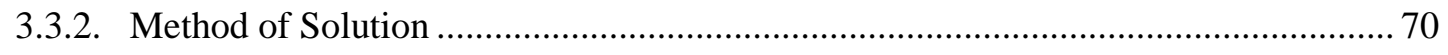

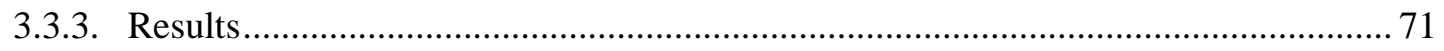

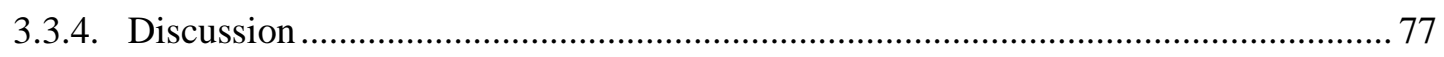

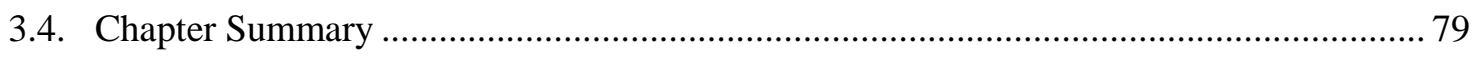

4. APPLICATIONS OF THE COMPRESSIBLE ACTUATOR DISK THEORY -

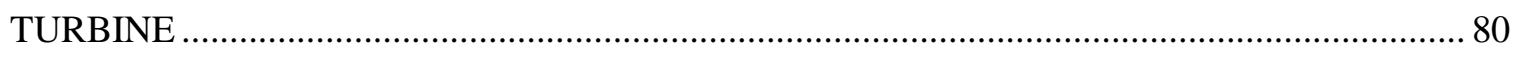

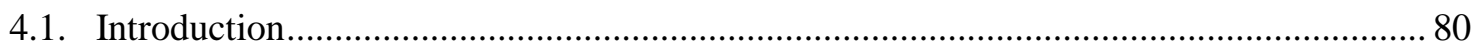

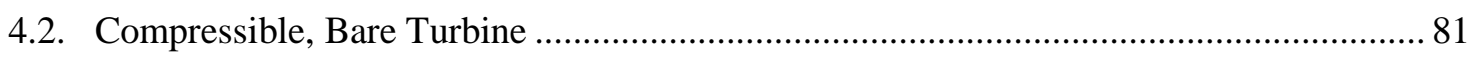

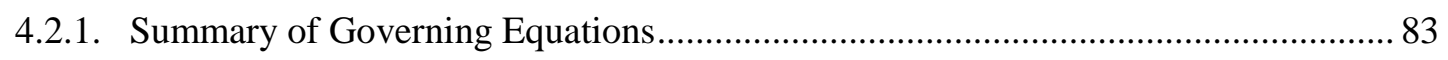




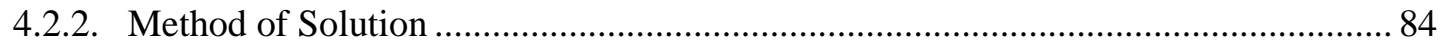

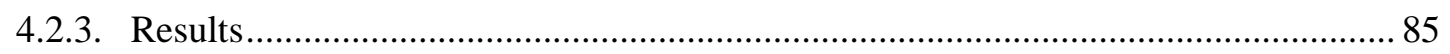

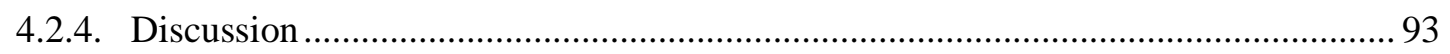

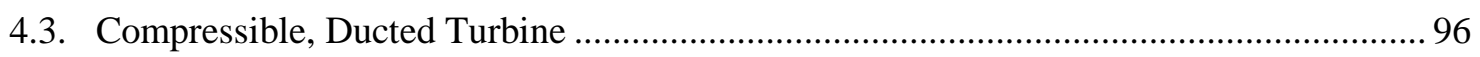

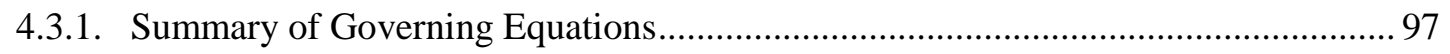

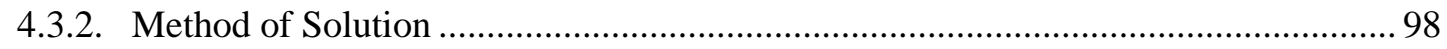

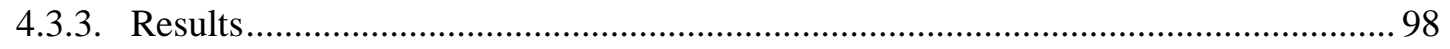

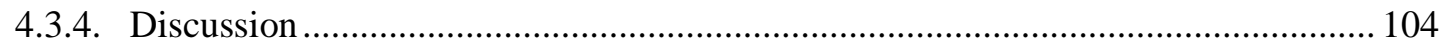

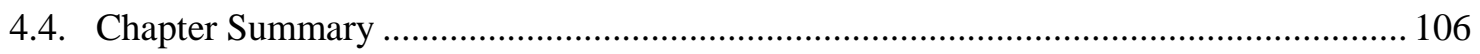

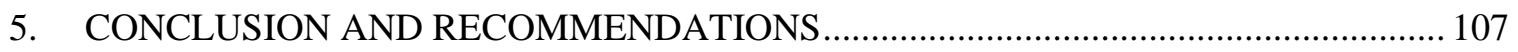

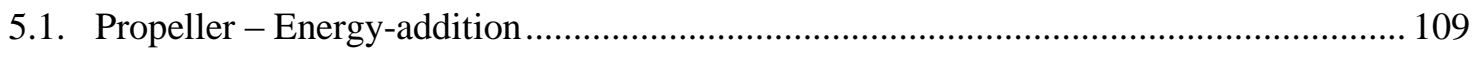

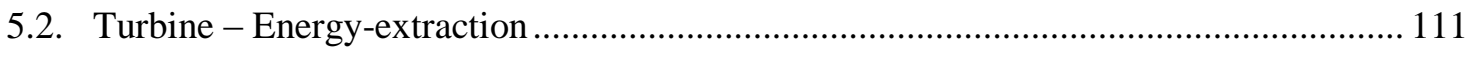

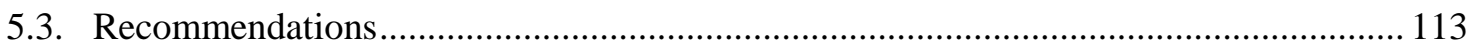

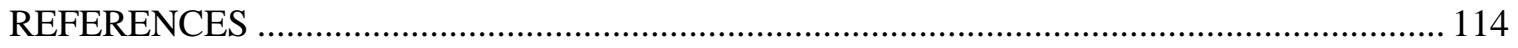

\section{APPENDICES}

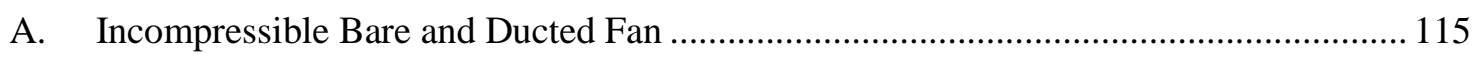

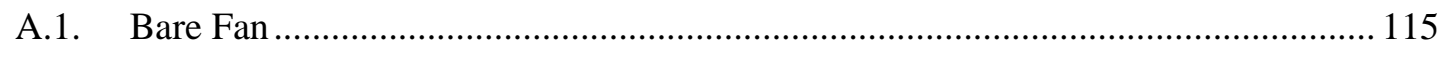

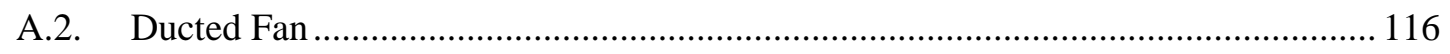

A.3. Results and Discussion - Incompressible Bare and Ducted Fan ........................... 117

B. Lip Thrust Analysis of the Compressible, Ducted Actuator Disk Theory ...................... 120

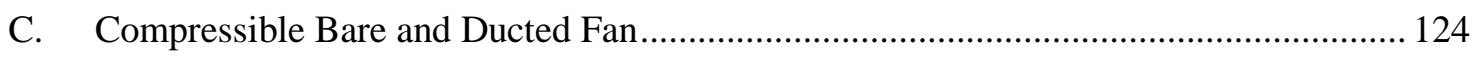

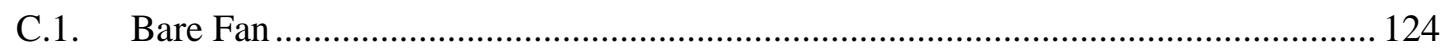

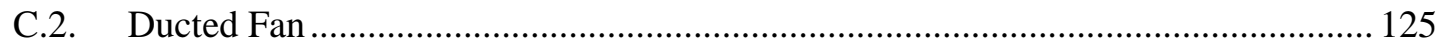


C.3. Results and Discussion - Compressible Bare and Ducted Fan.

D. EES Sheets - Compressible Bare Propeller.............................................................. 133

E. EES Sheets - Compressible Ducted Propeller ......................................................... 139

F. EES Sheets - Compressible Bare Turbine ............................................................. 145

G. EES Sheets - Compressible Ducted Turbine …....................................................... 151 


\section{LIST OF TABLES}

Page

Table 1. Table of given free-stream pressure and density and the disk area................................57

Table 2. Table of flow solutions of the bare propeller obtained at the maximum power coefficient before the flow is sonic at station 1 for various free-stream Mach numbers. Note that $*$ means incompressible flow values.

Table 3. Table of the Betz limit for the bare turbine between compressible and incompressible flow. .86 


\section{LIST OF FIGURES}

Figure 1. Schematic of the bare, incompressible Actuator Disk Theory. ......................................5

Figure 2. Schematic of the ducted, incompressible Actuator Disk Theory .................................. 9

Figure 3. Control volume used to drive lip thrust................................................................... 11

Figure 4. Propulsive efficiency versus the power coefficient between the ducted and

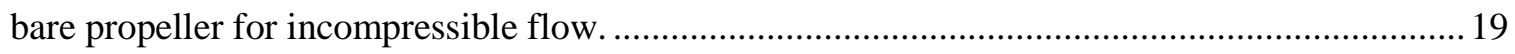

Figure 5. Comparison of the pressure rise across the disk between the bare and ducted propeller for incompressible flow.

Figure 6. Comparison of the total thrust between the bare and ducted propeller for incompressible flow.

Figure 7. The individual contribution of the propeller thrust and the lip thrust to the total thrust for the incompressible ducted propeller..

Figure 8. Velocity and pressure across the stations for the bare propeller (left) and the ducted propeller (right).

Figure 9. Comparison of the power extraction efficiency and the Betz limit between the bare and ducted turbine for incompressible flow.

Figure 10. Comparison of the pressure drop across the disk between the bare and ducted turbine for incompressible flow.

Figure 11. Comparison of the total drag between the bare and ducted turbine for incompressible flow.

Figure 12. The individual contribution of the turbine drag and the lip thrust to the total drag for the incompressible ducted turbine. Note that the total drag and the turbine drag are made negative to signify that they are in the opposite direction of the flow. 
Figure 13. Velocity and pressure throughout the stations for the bare turbine (left) and for the ducted turbine (right).

Figure 14. Schematic of the bare, compressible Actuator Disk Theory. ...................................... 34

Figure 15. Schematic of the ducted, compressible Actuator Disk Theory................................... 41

Figure 16. Control volume used to drive lip thrust. ............................................................... 44

Figure 17. The plot gives the maximum power coefficient to drive the propeller before the flow is sonic at station 1 against the free-stream Mach number for the bare propeller. .58

Figure 18. Propulsive efficiency of the bare propeller for incompressible flow and compressible flow of varying free-stream Mach numbers...... .58

Figure 19. Comparison of the total thrust coefficient between incompressible flow and compressible flow of varying free-stream Mach numbers for the bare propeller.

Figure 20. Comparison of the dimensionless mass flowrate between incompressible flow and compressible flow of varying free-stream Mach numbers for the bare propeller.

Figure 21. Comparison of the far upstream cross-sectional area between incompressible flow and compressible flow of varying free-stream Mach numbers for the bare propeller.

Figure 22. Comparison of the far downstream cross-sectional area between incompressible flow and compressible flow of varying free-stream Mach numbers for the bare propeller.

Figure 23. Comparison of the far downstream velocity between varying free-stream Mach numbers for the bare propeller.

Figure 24. Comparison of the far downstream Mach number between varying freestream Mach numbers for the bare propeller. 
Figure 25. Comparison of the velocities immediately before and after the bare propeller for varying free-stream Mach numbers.

Figure 26. Comparison of the Mach numbers immediately before and after the bare propeller for varying free-stream Mach numbers.

Figure 27. Comparison of the densities immediately before and after the bare propeller for varying free-stream Mach numbers.

Figure 28. Comparison of the pressures immediately before and after the bare propeller for varying free-stream Mach numbers.

Figure 29. Comparison of the pressure rise across the bare propeller for incompressible flow and compressible flow of varying free-stream Mach numbers.

Figure 30. Comparison of the velocities throughout the streamtube between incompressible and compressible flow of the same free-stream velocity for the bare propeller.

Figure 31. Comparison of the pressures immediately before and after the bare propeller between incompressible and compressible flow of the same free-stream velocity.

Figure 32. The plot gives the maximum power coefficient to drive the propeller before the flow is sonic at station 1 against the free-stream Mach number for the ducted propeller.

Figure 33. Propulsive efficiency of the bare and ducted propeller for incompressible flow and compressible flow of varying free-stream Mach numbers

Figure 34. Comparison of the dimensionless mass flowrate between incompressible flow and compressible flow of varying free-stream Mach numbers for the ducted propeller. 
Figure 35. Comparison of the far upstream cross-sectional area for incompressible flow and compressible flow of varying free-stream Mach numbers for the ducted propeller.

Figure 36. Comparison of the velocity immediately after the ducted propeller for incompressible flow and compressible flow of varying free-stream Mach numbers. Note that $V_{2}=V_{3}$ .73

Figure 37. Comparison of the Mach number immediately after the ducted propeller for varying free-stream Mach numbers. Note that $M_{2}=M_{3}$

Figure 38. Contribution of the propeller thrust and lip thrust to the total thrust at various freestream Mach numbers for incompressible and compressible flow.

Figure 39. Comparison of the velocity immediately before the ducted propeller for incompressible flow and compressible flow of varying free-stream Mach numbers.

Figure 40. Comparison of the Mach number immediately before the ducted propeller for varying free-stream Mach numbers.

Figure 41. Comparison of the density immediately before the ducted propeller for varying free-stream Mach numbers.

Figure 42. Comparison of the pressure immediately before the ducted propeller for incompressible flow and compressible flow of varying free-stream Mach numbers.

Figure 43. Comparison of the pressure rise across the disk for incompressible flow and compressible flow of varying free-stream Mach number for the ducted propeller. 77

Figure 44. Comparison of the bare turbine's power extraction efficiency for incompressible flow and for compressible flow of varying free-stream Mach numbers. 86

Figure 45. Comparison of the Betz Limit of the bare turbine for incompressible flow and for compressible flow of varying free-stream Mach numbers. The black dots 
represent the Betz Limit of each curve: note that as $M_{0}$ increases, the black dot slightly moves higher and more to the left.

Figure 46. The Betz Limit (maximum power extraction efficiency) versus the freestream Mach number $\mathrm{M}_{0}$ for the compressible, bare turbine.

Figure 47. The Betz Limit (maximum power extraction efficiency) versus the corresponding velocity ratio $\mathrm{r}$ for the compressible, bare turbine.

Figure 48. Comparison of the bare turbine's total drag coefficient for incompressible flow and for compressible flow of varying free-stream Mach numbers. 88

Figure 49. Comparison of the bare turbine's total drag coefficient for incompressible flow and for compressible of varying free-stream Mach numbers within a smaller domain and range (enlarged).

Figure 50. Comparison of the bare turbine's dimensionless mass flowrate for incompressible flow and compressible flow of varying free-stream Mach numbers.

Figure 51. Comparison of the area at the far downstream between incompressible flow and compressible flow of varying free-stream Mach numbers for the bare turbine.

Figure 52. Comparison of the area at the far upstream between incompressible flow and compressible flow of varying free-stream Mach numbers for the bare turbine. Note that $\frac{A_{0}}{A_{\text {disk }}}$ approaches infinity as $r$ goes to zero.

Figure 53. Comparison of the Mach numbers immediately before and after the bare turbine for varying free-stream Mach numbers. Note that $M_{2}$ surpasses $M_{0}$ between an $\mathrm{r}$ value of 0.5 and 0.55 for the curve $\mathrm{M}_{0}=0.8$.

Figure 54. Comparison of velocities immediately before and after the bare turbine for incompressible flow and compressible flow of varying free-stream Mach numbers. Note that $V_{2}$ surpasses $V_{0}$ between an $r$ value of 0.6 and 0.65 for the curve $M_{0}=0.8$. 
Figure 55. Comparison of the densities immediately before and after the bare turbine for varying free-stream Mach numbers.

Figure 56. Comparison of the pressures immediately before and after the bare turbine for compressible flow and incompressible flow of varying free-stream Mach numbers.

Figure 57. Comparison of the pressure drop across the bare turbine for incompressible flow and compressible flow of varying free-stream Mach numbers.

Figure 58. Comparison of the power extraction efficiency between the bare and the ducted turbine for incompressible flow and compressible of varying free-stream Mach numbers.

Figure 59. Contribution of the turbine drag and the lip thrust to the total drag for incompressible flow and for compressible flow of varying free-stream Mach numbers. Note that the total drag and the turbine drag are made negative to signify that they are in the opposite direction of the flow.....

Figure 60. Comparison of the dimensionless mass flowrate of the ducted turbine between incompressible flow and compressible flow of varying free-stream Mach numbers.

Figure 61. Comparison of the cross-sectional free-stream area of the ducted turbine for incompressible flow and compressible flow of varying free-stream Mach numbers. 101

Figure 62. Comparison of the Mach numbers immediately before the ducted turbine for varying free-stream Mach numbers. Note that $M_{2}=M_{3}$

Figure 63. Comparison of velocities immediately before the ducted turbine for incompressible flow and compressible flow of varying free-stream Mach numbers. Note that $V_{2}=V_{3}$ 102 
Figure 64. Comparison of the densities immediately before the ducted turbine for varying free-stream Mach numbers.

Figure 65. Comparison of the pressures immediately before the ducted turbine for incompressible and compressible flow of varying free-stream Mach numbers.

Figure 66. Comparison of the pressure drop across the ducted turbine for incompressible and compressible flow of varying free-stream Mach numbers. 103

Figure 67. Comparison of mass flowrate between the incompressible ducted and bare fan for two different disk cross-sectional areas. Note that the curves for the ducted fan with a disk area of $6 \mathrm{ft}^{2}$ and the bare fan with a disk area of $3 \mathrm{ft}^{2}$ are on top of each other. 118

Figure 68. Comparison of the total thrust between the incompressible ducted and bare fan for two different disk cross-sectional areas. Note that the curves for the ducted fan with a disk area of $6 \mathrm{ft}^{2}$ and the bare fan with a disk area of $3 \mathrm{ft}^{2}$ are on top of each other.

Figure 69. Comparison of the total thrust, fan thrust, and the lip thrust of the incompressible ducted fan for a disk cross-sectional area of $6 \mathrm{ft}^{2}$. Note that the curves for the fan thrust and the lip thrust are on top of each other.

Figure 70. Comparison of the mass flowrate between the compressible ducted and bare fan at two difference disk areas.

Figure 71. Comparison of the total thrust between the compressible ducted and bare fan at two different disk areas.

Figure 72. Comparison of the mass flowrate between the bare compressible and incompressible fan at two different disk areas.

Figure 73. Comparison of the total thrust between the bare compressible and incompressible fan at two different disk areas. 
Figure 74. Comparison of the mass flowrate between the ducted incompressible and compressible fan at two different disk areas.

Figure 75. Comparison of the total thrust between the ducted compressible and incompressible fan at two different disk areas......

Figure 76. Comparison of the total, fan, and lip thrust between the ducted compressible and incompressible fan at a disk area of $6 \mathrm{ft}^{2}$. 132 


\section{NOMENCLATURE}

a

A

$\mathrm{A}_{\text {disk }}$

$\mathrm{C}_{\mathrm{D}} \quad=\quad$ total drag coefficient; $\mathrm{C}_{\mathrm{D}}=\frac{\mathrm{D}_{\text {total }}}{\frac{1}{2} \rho_{0} \mathrm{~V}_{0}^{2} \mathrm{~A}_{\text {disk }}}$

$\mathrm{C}_{\mathrm{P}} \quad=\quad$ power coefficient $; \mathrm{C}_{\mathrm{p}}=\frac{\mathrm{P}_{\text {input }}}{\mathrm{P}_{\text {available }}}$

$\mathrm{C}_{\mathrm{T}} \quad=\quad$ total thrust coefficient; $\mathrm{C}_{\mathrm{T}}=\frac{\mathrm{T}_{\text {total }}}{\frac{1}{2} \rho_{0} \mathrm{~V}_{0}^{2} \mathrm{~A}_{\text {disk }}}$

$\mathrm{D} \quad=\quad \operatorname{drag}, \mathrm{lbf}$

$\mathrm{D}_{\text {total }} \quad=\quad$ total drag on duct-and-turbine assembly, lbf

E $\quad=\quad$ total energy per unit mass, ft-lbf/slug

$\dot{\mathrm{m}} \quad=\quad$ mass flowrate, $\mathrm{slug} / \mathrm{s}$

$\mathrm{M} \quad=\quad$ Mach number; $\mathrm{M}=\frac{\mathrm{V}}{\mathrm{a}}$

$\mathrm{P}_{\mathrm{t}} \quad=\quad$ total pressure, $\mathrm{lbf} / \mathrm{ft}^{2}$

$\mathrm{P} \quad=\quad$ static pressure, $\mathrm{lbf} / \mathrm{ft}^{2}$

$\mathrm{P}_{\text {available }} \quad=\quad$ total power available in flow in an area equal to the disk

area, ft-lbf/s

$\mathrm{P}_{\text {extracted }} \quad=\quad$ total power extracted from flow by actuator disk, $\mathrm{ft}-\mathrm{lbf} / \mathrm{s}$

$\mathrm{P}_{\text {input }} \quad=\quad$ total power added to flow by actuator disk, $\mathrm{ft}-\mathrm{lbf} / \mathrm{s}$

$\mathrm{r} \quad$ far up-to-downstream velocity ratio; $\frac{\mathrm{V}_{0}}{\mathrm{~V}_{3}}$

$\mathrm{T} \quad$ = $\quad$ thrust, $\mathrm{lbf}$

$\mathrm{T}_{\text {lip }} \quad=\quad$ lip thrust, lbf (ducted case only)

$\mathrm{T}_{\text {total }}=\quad$ total thrust on duct-and-disk assembly, lbf

$\mathrm{V} \quad=\quad$ velocity, $\mathrm{ft} / \mathrm{s}$ 


\begin{tabular}{|c|c|c|}
\hline$\gamma$ & $=$ & ratio of specific heats ( 1.4 for air) \\
\hline$\rho$ & $=$ & density, slug/ft ${ }^{2}$ \\
\hline$\eta_{\text {fan }}$ & $=$ & fan efficiency \\
\hline$\eta_{\text {prop }}$ & $=$ & propulsive efficiency \\
\hline$\eta_{\text {turb }}$ & $=$ & extraction efficiency \\
\hline \multicolumn{3}{|c|}{ Subscripts: } \\
\hline 0 & $=$ & far upstream \\
\hline 1 & $=$ & immediate upstream of actuator disk \\
\hline 2 & $=$ & immediate downstream of actuator disk \\
\hline 3 & $=$ & far downstream \\
\hline $\mathrm{i}$ & $=$ & duct inlet \\
\hline $\mathrm{e}$ & $=$ & duct exit \\
\hline disk & $=$ & actuator disk \\
\hline fan & $=$ & fan \\
\hline prop & $=$ & propeller \\
\hline turb & $=$ & turbine \\
\hline
\end{tabular}




\section{CLASSICAL ACTUATOR DISK THEORY}

\subsection{Introduction}

The classical Actuator Disk Theory, also known as Froude's Momentum Theory, is a mathematical model that uses the conservation laws of mass, linear momentum, and energy to determine the ideal performance of an energy-adding or an energy-extracting device, such as a propeller or a turbine, in a flow stream that is steady, inviscid, one-dimensional, and incompressible. Such work has been explored intensively by Horlock [1], Glauert [2], and Küchemann and Weber [3], but much less has been done to apply the Actuator Disk Theory to compressible flow. Since compressibility effects can arise in real applications of energy-adding or energy-extracting devices, the goal of the current thesis is to extend the Actuator Disk Theory into the regime of compressible flow, with the primary motivation to predict the ideal performance of a small ram-air turbine (microRAT) [4] for the applications of the Boundary Layer Data System (BLDS).

The Boundary Layer Data System (BLDS) is a fully autonomous, flow measurement system developed by Dr. Russell Westphal and his team of students with the purpose of measuring the flow properties of the boundary layer at the surface of an aircraft in flight. Since the electronic components of the BLDS, such as the microcontroller and the sensors, are not rated to perform at temperatures below $-20^{\circ} \mathrm{C}$, their performance diminishes and becomes unreliable during test flights at altitudes between 30,000 and 40,000 feet where the air temperature ranges from $-40{ }^{\circ} \mathrm{C}$ to $-57{ }^{\circ} \mathrm{C}$ or below. To prevent this sensor drop out, the concept of using a small ram-air turbine (microRAT) to extract useful energy from the airflow to provide power to a heating element that maintains operable temperatures inside the BLDS was investigated by Victor Villa [4]. Because the microRAT will be operating at high subsonic Mach numbers where compressibility effects exist, the current thesis will develop the governing equations of the compressible Actuator Disk Theory that can be used to determine the ideal performance of the microRAT. 
Such governing equations of the Actuator Disk Theory will be derived for compressible, subsonic flow using isentropic thermodynamics and the three laws of conservation while adopting an iterative method of solution demonstrated by Delano and Crigler [5]. The governing compressible-flow equations are established for two cases: the first is the bare or the unducted case where the actuator disk is treated as the only assembly within the flow stream, while the latter is the ducted case where the disk is enclosed by a duct. The governing equations of the compressible Actuator Disk Theory are then applied to determine the theoretical performance of a propeller and a turbine for both the bare and ducted cases. The application of the compressible Actuator Disk Theory to the bare propeller has previously been studied by Delano and Crigler [5] and is included in the current thesis to show agreement and to confirm the results of the bare and ducted turbine in compressible flow, which is the main interest of the current thesis.

Before the development of the governing equations for the compressible Actuator Disk Theory, the current chapter will introduce the classical (incompressible) Actuator Disk Theory and its applications to a propeller and a turbine with and without a duct. Even though the classical Actuator Disk Theory and its applications can be found in many Fluid Mechanics textbooks, it is included in this thesis for completeness and to provide a basis for comparison between the classical and compressible Actuator Disk Theory. 


\subsection{Classical Actuator Disk Theory - Governing Equations}

The classical Actuator Disk Theory, also known as Froude's Momentum Theory, uses the conservation of mass, linear momentum, and energy to determine the theoretical performance of an energy-adding or energy-extracting device in a flow that is considered steady, inviscid, onedimensional, and incompressible. The energy-adding or energy-extracting device is represented by an ideal actuator disk having a cross-sectional area of $\mathrm{A}_{\text {disk }}$. The ideal actuator disk is assumed to be infinitely thin and permeable such that the flow can pass through its cross-sectional area fully undisrupted thus producing no swirls in the slipstream. The energy addition to or extraction from the flow occurs instantaneously and uniformly throughout the disk's crosssectional area as the flow passes through the disk.

Two cases of the actuator disk are considered: the bare and the ducted. The bare, or unducted, case is where the actuator disk is the only assembly in the flow stream as shown in Figure 1, while the ducted case is where the actuator disk is enclosed by a duct as shown in Figure 2. The streamtube boundary containing the actuator disk in both cases, as shown in Figure 1 and Figure 2, is described by four stations: stations 0 and 3 denote the far upstream and downstream of the disk, respectively, where the free-stream density and pressure exists. Stations 1 and 2 are immediately before and after the disk.

The complete list of assumptions made for the classical Actuator Disk Theory are given in the next section. Note that these assumptions, with the exception of the first and second, are also applicable to the compressible Actuator Disk Theory.

\subsubsection{List of Assumptions}

1) The flow is incompressible.

2) For incompressible flow, the velocity across the actuator disk is continuous, but the pressure across the disk is discontinuous: $\mathrm{V}_{1}=\mathrm{V}_{2}$ and $\mathrm{P}_{1} \neq \mathrm{P}_{2}$. 
3) The flow is steady, inviscid, and one-dimensional.

4) The static pressure at the far upstream and downstream stations are equal: $P_{0}=P_{3}$.

5) The ideal actuator disk is an infinitely thin, permeable disk with a cross-sectional area of $\mathrm{A}_{\text {disk }}$ and is uniformly loaded and has a nonrotating wake.

6) The stream tube cross-sectional area is continuous throughout the disk such that the areas immediately before and after the disk are equal to the disk area: $A_{1}=A_{2}=A_{\text {disk }}$.

7) Energy is added to or extracted from the flow stream instantaneously and uniformly through the actuator disk.

List of Assumptions Specific to the Ducted Actuator Disk

8) The actuator disk is enclosed by a straight, constant area duct with the same crosssectional area as the disk area $A_{\text {disk }}$.

9) The duct produces straight and parallel streamlines at the duct exit that the exit pressure is considered equal to the far downstream pressure: $\mathrm{P}_{\mathrm{e}}=\mathrm{P}_{3}$.

Using these assumptions, the governing equations that are necessary to determine the ideal performance of the actuator disk will be developed in the next section. 


\subsubsection{Incompressible, Bare Actuator Disk Theory}

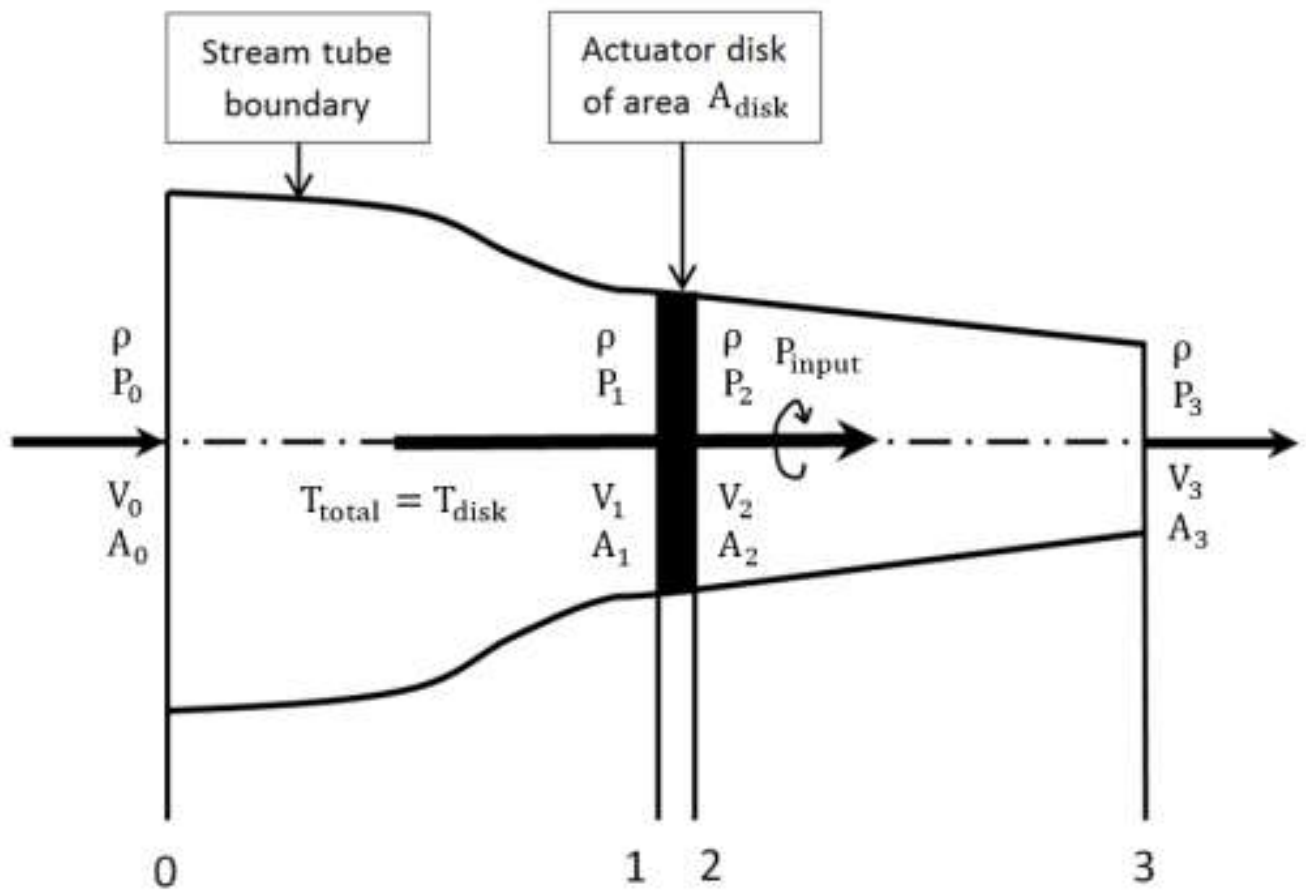

Figure 1. Schematic of the bare, incompressible Actuator Disk Theory.

The general governing equations of an ideal bare actuator disk will be established. Consider the actuator disk contained by the streamtube boundary as shown in Figure 1. The control volume 03 contains the flow from station 0 to 3 and the control volumes $0-1$ and 2-3 encloses the flow upstream and downstream of the disk, respectively. Lastly, the control volume 1-2 represents the flow through the disk plane.

It is assumed that the disk is an energy-adding device where it adds power to the flow stream at the disk plane and exerts a thrust force on the flow in the direction of the flow. It is possible that the disk is also a power-extractor where it extracts power from the flow and experiences a drag force from the flow, but the governing equations will be derived assuming the former. Thus, the performance parameters of interest are the total thrust experienced by the flow, the thrust exerted on the flow by the disk, the power added to the flow at the disk plane, and the mass flowrate throughout the streamtube. 
By applying the conservation of linear momentum to the control volume $0-3$, the total thrust experienced by the flow can be determined

$$
\mathrm{T}_{\text {total }}=\dot{\mathrm{m}}\left(\mathrm{V}_{3}-\mathrm{V}_{0}\right)
$$

Likewise, by applying the conversation of energy to the control volume $0-3$, the power added by the disk to the flow stream can be obtained

$$
\mathrm{P}_{\text {input }}=\frac{1}{2} \dot{\mathrm{m}}\left(\mathrm{V}_{3}^{2}-\mathrm{V}_{0}^{2}\right)
$$

To find the thrust produced by the disk, the momentum balance law is applied to the control volume 1-2. Since the velocity is equal before and after the disk, the momentum balance is reduced to an equilibrium equation. By summing the external axial forces at the disk plane gives

$$
\mathrm{T}_{\text {disk }}=\left(\mathrm{P}_{2}-\mathrm{P}_{1}\right) \mathrm{A}_{\text {disk }}
$$

Next, to determine the pressure difference across the disk on the right side of equation $1-3$, the Bernoulli's equation is applied to the control volumes $0-1$ and 2-3 as follows

$$
\begin{aligned}
& P_{0}+\frac{1}{2} \rho V_{0}^{2}=P_{1}+\frac{1}{2} \rho V_{1}^{2} \\
& P_{2}+\frac{1}{2} \rho V_{2}^{2}=P_{3}+\frac{1}{2} \rho V_{3}^{2}
\end{aligned}
$$

By subtracting equation 1-5 from 1-4 and asserting that $P_{0}=P_{3}$ and $V_{1}=V_{2}$, the pressure difference across the disk is

$$
\mathrm{P}_{2}-\mathrm{P}_{1}=\frac{1}{2} \rho\left(\mathrm{V}_{3}^{2}-\mathrm{V}_{0}^{2}\right)
$$

Substituting the pressure difference in equation 1-6 to equation 1-3 gives the disk thrust in terms of the far upstream and downstream velocities

$$
\mathrm{T}_{\text {disk }}=\frac{1}{2} \rho \mathrm{A}_{\text {disk }}\left(\mathrm{V}_{3}^{2}-\mathrm{V}_{0}^{2}\right)
$$

Because the mass flowrate is constant throughout the streamtube, the following relations can be made

$$
\dot{\mathrm{m}}=\rho \mathrm{A}_{0} \mathrm{~V}_{0}=\rho \mathrm{A}_{\text {disk }} \mathrm{V}_{1}=\rho \mathrm{A}_{3} \mathrm{~V}_{3}
$$


From the relations in equation 1-8 and canceling out the density, the far upstream and downstream cross-sectional areas can be found in terms of the disk velocity

$$
\begin{aligned}
& \mathrm{A}_{0}=\mathrm{A}_{\text {disk }} \frac{\mathrm{V}_{1}}{\mathrm{~V}_{0}} \\
& \mathrm{~A}_{3}=\mathrm{A}_{\text {disk }} \frac{\mathrm{V}_{1}}{\mathrm{~V}_{3}}
\end{aligned}
$$

For the bare case of the classical Actuator Disk Theory, the disk is the only assembly within the streamtube boundary, so the total thrust $\mathrm{T}_{\text {total }}$ exerted on the flow is equal to the thrust produced at the disk $\mathrm{T}_{\text {disk }}$. Note that this can only be said for the bare disk.

$$
\mathrm{T}_{\text {total }}=\mathrm{T}_{\text {disk }}
$$

By setting the disk thrust in equation 1-7 and the total thrust in equation 1-1 equal to each other and using the mass flowrate containing the disk area and velocity in equation $1-8, V_{1}$ is found to be the average of the far upstream and downstream velocities

$$
\begin{gathered}
\frac{1}{2} \rho A_{\text {disk }}\left(V_{3}^{2}-V_{0}^{2}\right)=\dot{m}\left(V_{3}-V_{0}\right) \\
V_{1}=\frac{V_{0}+V_{3}}{2}
\end{gathered}
$$

Lastly, by substituting equation $1-13$, the mass flowrate can be put in terms of the far upstream and downstream velocities

$$
\dot{\mathrm{m}}=\rho \mathrm{A}_{\mathrm{disk}} \frac{\mathrm{V}_{0}+\mathrm{V}_{3}}{2}
$$

thus concluding the analysis. The governing equations for the bare actuator disk are further simplified and rearranged to give the following flow variables in terms of the disk area, freestream density and pressure, and the far upstream and downstream velocities:

$$
\begin{gathered}
\mathrm{T}_{\text {total }}=\dot{\mathrm{m}}\left(\mathrm{V}_{3}-\mathrm{V}_{0}\right) \\
\mathrm{T}_{\text {disk }}=\frac{1}{2} \rho \mathrm{A}_{\text {disk }}\left(\mathrm{V}_{3}^{2}-\mathrm{V}_{0}^{2}\right) \\
\mathrm{P}_{\text {disk }}=\frac{1}{2} \dot{\mathrm{m}}\left(\mathrm{V}_{3}^{2}-\mathrm{V}_{0}^{2}\right) \\
\dot{\mathrm{m}}=\rho \mathrm{A}_{\text {disk }}\left(\frac{\mathrm{V}_{0}+\mathrm{V}_{3}}{2}\right)
\end{gathered}
$$




$$
\begin{gathered}
\mathrm{A}_{0}=\mathrm{A}_{\text {disk }} \frac{\mathrm{V}_{1}}{\mathrm{~V}_{0}} \\
\mathrm{~A}_{3}=\mathrm{A}_{\text {disk }} \frac{\mathrm{V}_{1}}{\mathrm{~V}_{3}} \\
\mathrm{P}_{1}=\mathrm{P}_{0}+\frac{1}{2} \rho\left(\mathrm{V}_{0}^{2}-\mathrm{V}_{1}^{2}\right) \\
\mathrm{P}_{2}=\mathrm{P}_{0}+\frac{1}{2} \rho\left(\mathrm{V}_{3}^{2}-\mathrm{V}_{1}^{2}\right) \\
\mathrm{V}_{1}=\frac{\mathrm{V}_{0}+\mathrm{V}_{3}}{2}
\end{gathered}
$$

Note that equations 1-19 to 1-22 can be expressed in terms of the far upstream and downstream velocities with the substitution of equation 1-23 for $V_{1}$ but are left in the current form for simplicity. Similarly, the governing equations will be developed for the ducted disk in the next section. 


\subsubsection{Incompressible, Ducted Actuator Disk Theory}

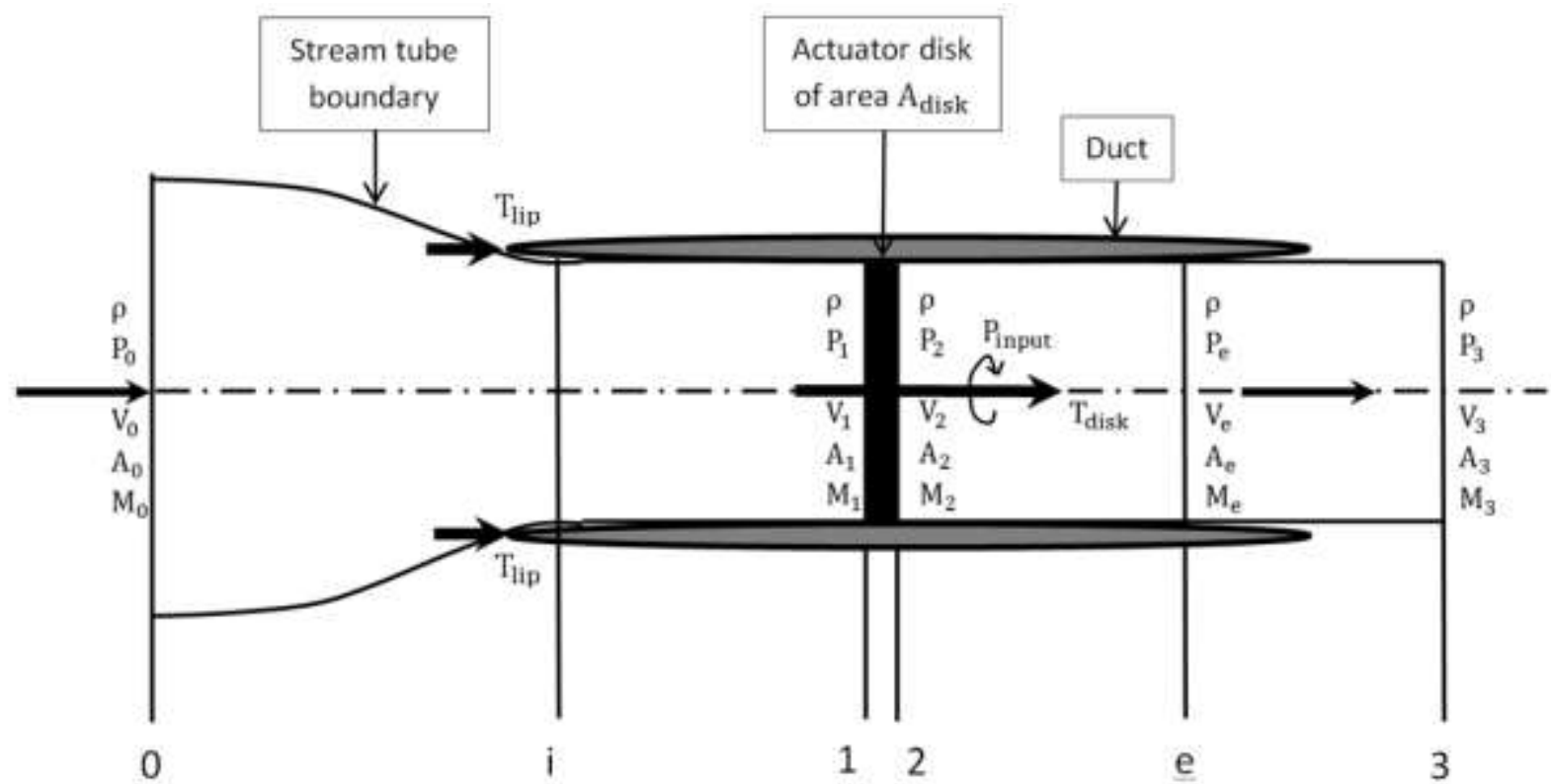

Figure 2. Schematic of the ducted, incompressible Actuator Disk Theory.

The governing equations for the ducted case of the incompressible Actuator Disk Theory will be established. Note that the assumptions listed in section 1.2.1 also apply to the ducted actuator disk. It is again assumed that the disk adds power to the flow stream and exerts a thrust force on the flow in the direction of the flow. The disk is enclosed by a duct with a constant crosssectional area equal to the disk area $A_{\text {disk }}$ as shown in Figure 2, where stations $i$ and e are the duct inlet and outlet. While it is certainly possible that the duct is also of variable cross-sectional area, it is assumed to have constant area to keep the scope of the thesis manageable. The constant area duct implies that the areas at the duct inlet and outlet and the far downstream are equal to the disk area

$$
A_{i}=A_{1}=A_{2}=A_{\text {disk }}=A_{e}=A_{3}
$$

It is also assumed that the duct produces straight and parallel streamlines at the duct exit such that the exit pressure is equal to the far downstream pressure, which in turn is equal to the free-stream pressure. 


$$
\mathrm{P}_{0}=\mathrm{P}_{\mathrm{e}}=\mathrm{P}_{3}
$$

From the conservation of mass and using the area relation in equation 1-24, it can also be observed that the velocities at the disk, the duct outlet, and the far downstream are equal.

$$
\begin{aligned}
\rho \mathrm{A}_{\text {disk }} \mathrm{V}_{1} & =\rho \mathrm{A}_{\mathrm{e}} \mathrm{V}_{\mathrm{e}}=\rho \mathrm{A}_{3} \mathrm{~V}_{3} \\
\mathrm{~V}_{1} & =\mathrm{V}_{\mathrm{e}}=\mathrm{V}_{3}
\end{aligned}
$$

Therefore, since the area and all the flow variables, which are the density, pressure, and velocity, at the duct exit and the far downstream are equal; station e is entirely replaced by station 3 for the rest of the analysis. As a result, the governing equations 1-15 to 1-22 derived for the bare actuator disk also applies to the ducted disk without any change of subscripts since the control volumes 0 3, 1-2, and 2-3 encloses the same stations for both the bare and ducted actuator disk.

Furthermore, with the assertion that $V_{1}=V_{2}=V_{3}$, it can be seen from the Bernoulli's equation applied to the control volume $2-3$ that the pressure immediately after the disk is equal to the far downstream pressure, which in turn is equal to the free-stream pressure.

$$
\begin{gathered}
\mathrm{P}_{2}+\frac{1}{2} \rho \mathrm{V}_{2}^{2}=\mathrm{P}_{3}+\frac{1}{2} \rho \mathrm{V}_{3}^{2} \\
\mathrm{P}_{0}=\mathrm{P}_{2}=\mathrm{P}_{3}
\end{gathered}
$$

Due to the addition of the constant area duct onto the disk assembly, $\mathrm{T}_{\text {total }}$ given in equation 1-15 now signifies the total thrust that the disk and the duct exerts altogether on the flow, while $\mathrm{T}_{\text {disk }}$ in equation 1-16 represents the thrust exerted only by the disk. The duct lip also exerts an additional thrust force on the flow called the lip thrust [2] [6].

Küchemann and Weber [3] and later Greitzer [6] developed the lip thrust by applying the conservation of linear momentum to the control volume enclosing the far upstream and the duct inlet as shown in Figure 3. The far upstream is denoted by the subscript 0 while the inlet, which is approximated as being a constant section some distance behind the lip, is denoted by the subscript i. 


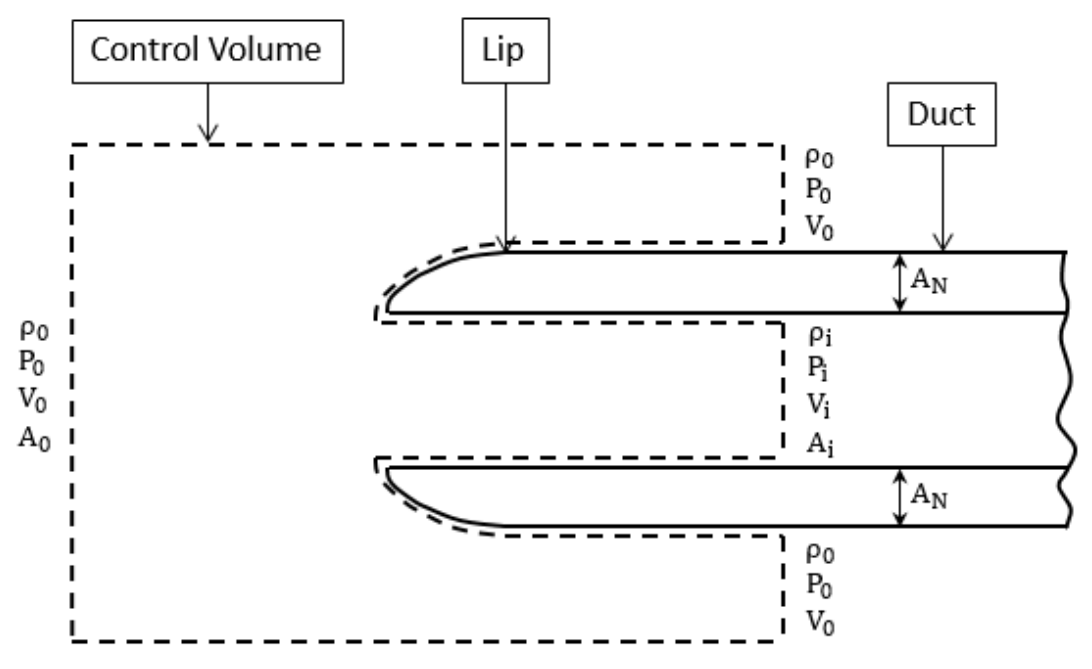

Figure 3. Control volume used to drive lip thrust.

The resulting integral momentum equation applied to the control volume in Figure 3 is

$$
\begin{aligned}
& \rho_{0} V_{0}^{2} A_{0}+P_{0} A_{0}-\rho_{i} V_{i}^{2} A_{i}-P_{i} A_{i}-\left(\int_{A_{N}} P_{N} d A_{N}\right) \\
& -P_{0}\left(A_{0}-A_{i}-A_{N}\right)-\left(\rho_{0} V_{0} A_{0}-\rho_{i} V_{i} A_{i}\right) V_{0}=0
\end{aligned}
$$

As described by Kuchemann and Weber [3] and Grietzer [6], the first two terms on the left side of equation 1-30 represents the momentum of the mass flow, $\rho V_{0} A_{0}$, through the forward surface $A_{0}$ of the control volume and the pressure force $\mathrm{P}_{0}$ which acts on that surface. The third and fourth terms are the corresponding quantities for the flow through the internal duct. The fifth term is the integral of the static pressure $P_{N}$ over the surface of the duct inlet where $d A_{N}$ is a surface element normal to the direction of the flow. The sixth term represents the force on the base of the control volume outside of the duct inlet where the streamlines are assumed to be straight that the pressure is equal to the free-stream value. The last term is the momentum of the mass flow diverted through the outer part of the base of the control volume and through its curved surface of the duct inlet, with the control volume assumed large enough that the axial velocity component of this mass flow is $V_{0}$.

Cancelling out terms and simplifying equation 1-30 gives

$$
\int_{A_{N}}\left(P_{N}-P_{0}\right) d A_{N}=\rho_{i} V_{i} A_{i}\left(V_{0}-V_{i}\right)-\left(P_{i}-P_{0}\right) A_{i}
$$


Note that equation 1-31 is general and non-specific to the compressibility of the flow. For incompressible flow where $\rho_{0}=\rho_{\mathrm{i}}=\rho$, and using the following Bernoulli's equation from the far upstream to the duct inlet,

$$
\mathrm{P}_{\mathrm{i}}-\mathrm{P}_{0}=\frac{1}{2} \rho \mathrm{V}_{0}^{2}-\frac{1}{2} \rho \mathrm{V}_{\mathrm{i}}^{2}
$$

the equation in 1-31 is further reduced to

$$
\int_{A_{N}}\left(P_{N}-P_{0}\right) d A_{N}=\frac{1}{2} \rho V_{0}^{2} A_{i}\left(1-\frac{V_{i}}{V_{0}}\right)^{2}
$$

The lip thrust is the negative of the integral on the left side of equation 1-33

$$
T_{\text {lip }}=-\int_{A_{N}}\left(P_{N}-P_{0}\right) d A_{N}=\frac{1}{2} \rho V_{0}^{2} A_{i}\left(\frac{V_{i}}{V_{0}}-1\right)^{2}
$$

and it is the force that the duct lip exerts on the flow. Note that when the ratio of the duct inlet to the far upstream velocity $\frac{V_{i}}{V_{0}}$ is equal to 1 , the lip thrust goes to zero. Assuming the streamlines are straight and parallel so that the pressure immediately before the disk at station 1 is the same as the inlet value, the flow variables at station $i$ and 1 are then equal due to the same logic applied to stations e and 3 being equal. Therefore, replacing the subscript $i$ in equation 1-34 with 1 and asserting that $A_{1}=A_{\text {disk }}$ give

$$
\mathrm{T}_{\text {lip }}=\frac{1}{2} \rho \mathrm{V}_{0}^{2} \mathrm{~A}_{\text {disk }}\left(\frac{\mathrm{V}_{1}}{\mathrm{~V}_{0}}-1\right)^{2}
$$

Alternatively, the lip thrust can also be found by applying the static equilibrium equation on the disk-and-duct assembly in the direction of the flow, giving that the lip thrust is the difference between the total thrust and the disk thrust.

$$
\mathrm{T}_{\text {lip }}=\mathrm{T}_{\text {total }}-\mathrm{T}_{\text {disk }}
$$

By substituting the total thrust in 1-15 and the disk thrust in 1-16 into 1-36 and carrying out the simplification, the lip thrust from the difference of the total thrust and the disk thrust must equal the lip thrust determined by Küchemann and Weber [3]. This will be shown in the next following steps. 


$$
\mathrm{T}_{\text {lip }}=\rho \mathrm{A}_{\text {disk }} \mathrm{V}_{1}\left(\mathrm{~V}_{1}-\mathrm{V}_{0}\right)-\frac{1}{2} \rho \mathrm{A}_{\text {disk }}\left(\mathrm{V}_{1}^{2}-\mathrm{V}_{0}^{2}\right)
$$

One half of the density and the disk area can be factored out from both terms on the right side of equation 1-37 to give

$$
\mathrm{T}_{\text {lip }}=\frac{1}{2} \rho \mathrm{A}_{\text {disk }}\left(2\left(\mathrm{~V}_{1}^{2}-\mathrm{V}_{1} \mathrm{~V}_{0}\right)-\left(\mathrm{V}_{1}^{2}-\mathrm{V}_{0}^{2}\right)\right)
$$

where the terms inside the parentheses on the right side of equation 1-38 can be combined to give

$$
\mathrm{T}_{\text {lip }}=\frac{1}{2} \rho \mathrm{A}_{\text {disk }}\left(\mathrm{V}_{1}-\mathrm{V}_{0}\right)^{2}
$$

By factoring out $\mathrm{V}_{0}^{2}$ from inside of the parentheses gives the same form of the lip thrust in equation 1-35 as expected

$$
\mathrm{T}_{\text {lip }}=\frac{1}{2} \rho \mathrm{A}_{\text {disk }} \mathrm{V}_{0}^{2}\left(\frac{\mathrm{V}_{1}}{\mathrm{~V}_{0}}-1\right)^{2}
$$

Asserting that $V_{1}=V_{3}$, the lip thrust in equation 1-40 can be put in terms of the far upstream and downstream velocities

$$
\mathrm{T}_{\text {lip }}=\frac{1}{2} \rho \mathrm{A}_{\text {disk }} \mathrm{V}_{0}^{2}\left(\frac{\mathrm{V}_{3}}{\mathrm{~V}_{0}}-1\right)^{2}
$$

In summary, the assumption of constant duct area implies the following for the ducted case of the incompressible Actuator Disk Theory.

$$
\begin{gathered}
\mathrm{V}_{1}=\mathrm{V}_{\mathrm{e}}=\mathrm{V}_{3} \\
\mathrm{P}_{0}=\mathrm{P}_{2}=\mathrm{P}_{\mathrm{e}}=\mathrm{P}_{3}
\end{gathered}
$$

Due to these implications, the governing equations for the ducted actuator disk can be summarized in terms of the far upstream and downstream velocities as follows

$$
\begin{gathered}
\mathrm{T}_{\text {total }}=\dot{\mathrm{m}}\left(\mathrm{V}_{3}-\mathrm{V}_{0}\right) \\
\mathrm{T}_{\text {disk }}=\frac{1}{2} \rho \mathrm{A}_{\text {disk }}\left(\mathrm{V}_{3}^{2}-\mathrm{V}_{0}^{2}\right) \\
\mathrm{T}_{\text {lip }}=\frac{1}{2} \rho \mathrm{A}_{\text {disk }} \mathrm{V}_{0}^{2}\left(\frac{\mathrm{V}_{3}}{\mathrm{~V}_{0}}-1\right)^{2} \\
\mathrm{P}_{\text {input }}=\frac{1}{2} \dot{\mathrm{m}}\left(\mathrm{V}_{3}^{2}-\mathrm{V}_{0}^{2}\right) \\
\dot{\mathrm{m}}=\rho \mathrm{A}_{\text {disk }} \mathrm{V}_{3}
\end{gathered}
$$




$$
\begin{gathered}
A_{0}=A_{\operatorname{disk}} \frac{V_{3}}{V_{0}} \\
P_{1}=P_{0}+\frac{1}{2} \rho\left(V_{0}^{2}-V_{3}^{2}\right)
\end{gathered}
$$

The governing equations for the bare and ducted actuator disk are applied in the next sections to determine the ideal performance of a propeller and a turbine. 


\subsection{Applications of the Classical Actuator Disk Theory}

The governing equations of the bare and ducted Actuator Disk Theory are applied to the propeller and the turbine. The purpose of a propeller is to produce high propulsive thrust for a given power input while the purpose of a turbine is to extract power from the total kinetic energy of the flow stream to generate useful work for other applications. Due to these reasons, a popular figure of merit for a propeller is its propulsive efficiency while it is the power extraction efficiency for a turbine. The equations for the figure of merit and other important performance parameters will be established for the propeller and the turbine in the following sections, beginning with the bare propeller.

\subsubsection{Incompressible Bare Propeller}

The performance parameters of the bare propeller will be established using the governing equations derived for the bare incompressible Actuator Disk Theory. The performance parameters considered are the power coefficient, the total thrust coefficient, and the propulsive efficiency. Note that since the governing equations in section 1.2.2 are derived already assuming that the actuator disk is an energy-adding device, much like a propeller, the equations can be transferred over to the current analysis without any manipulation or sign change.

The power coefficient is determined by dividing the power input to the propeller given in equation 1-17 by the total available power in the free-stream in an area of $A_{\text {disk }}$,

$$
C_{P}=\frac{P_{\text {input }}}{P_{\text {available }}}=\frac{\frac{1}{2} \dot{m}\left(V_{3}^{2}-V_{0}^{2}\right)}{\frac{1}{2} \rho A_{\text {disk }} V_{0}^{3}}
$$

By using the mass flowrate given in equation 1-18 and the disk velocity given in equation 1-23, the power coefficient can be simplified to the following

$$
C_{P}=\frac{1}{2}\left(\frac{V_{3}}{V_{0}}+1\right)\left(\left(\frac{V_{3}}{V_{0}}\right)^{2}-1\right)
$$


Likewise, the total thrust coefficient is determined by dividing the total thrust, which is equal to the disk thrust in equation 1-16, by the reference force associated with the free-flowing stream's dynamic pressure and the disk area.

$$
\mathrm{C}_{\mathrm{T}}=\frac{\mathrm{T}_{\text {total }}}{\mathrm{T}_{\text {dynamic }}}=\frac{\frac{1}{2} \rho \mathrm{A}_{\text {disk }}\left(\mathrm{V}_{3}^{2}-\mathrm{V}_{0}^{2}\right)}{\frac{1}{2} \rho \mathrm{A}_{\text {disk }} \mathrm{V}_{0}^{2}}
$$

The total thrust coefficient in equation 1-53 can be simplified further to the following

$$
\mathrm{C}_{\mathrm{T}}=\left(\left(\frac{\mathrm{V}_{3}}{\mathrm{~V}_{0}}\right)^{2}-1\right)
$$

Next, the propulsive efficiency is the ratio of the propulsive power produced by the propeller to the total power added to the propeller. The propulsive power is given by the product of the total thrust and the free-stream velocity

$$
\eta_{\text {prop }}=\frac{\text { propulsive power }}{P_{\text {input }}}=\frac{\dot{\mathrm{m}}\left(\mathrm{V}_{3}-\mathrm{V}_{0}\right) \mathrm{V}_{0}}{\frac{1}{2} \dot{\mathrm{m}}\left(\mathrm{V}_{3}^{2}-\mathrm{V}_{0}^{2}\right)}
$$

By simplifying, the propulsive efficiency is given in terms of the velocity ratio $\frac{V_{3}}{V_{0}}$

$$
\eta_{\text {prop }}=\frac{2 \mathrm{~V}_{0}}{\mathrm{~V}_{3}+\mathrm{V}_{0}}=\frac{2}{\frac{\mathrm{V}_{3}}{\mathrm{~V}_{0}}+1}
$$

Alternatively, the propulsive efficiency is also the ratio of the total thrust coefficient to the power coefficient

$$
\eta_{\text {prop }}=\frac{C_{T}}{C_{p}}=\frac{\left(\left(\frac{V_{3}}{V_{0}}\right)^{2}-1\right)}{\frac{1}{2}\left(\frac{V_{3}}{V_{0}}+1\right)\left(\left(\frac{V_{3}}{V_{0}}\right)^{2}-1\right)}
$$

Simplifying equation 1-57 gives the same propulsive efficiency given in equation 1-56. The performance parameters of the bare propeller are summarized in terms of the far upstream and downstream velocities.

Dimensional Quantities:

$$
\begin{gathered}
\mathrm{T}_{\text {total }}=\dot{\mathrm{m}}\left(\mathrm{V}_{3}-\mathrm{V}_{0}\right) \\
\mathrm{T}_{\text {prop }}=\frac{1}{2} \rho \mathrm{A}_{\text {disk }}\left(\mathrm{V}_{3}^{2}-\mathrm{V}_{0}^{2}\right) \\
\mathrm{T}_{\text {total }}=\mathrm{T}_{\text {prop }}
\end{gathered}
$$




$$
\begin{gathered}
\mathrm{P}_{\text {input }}=\frac{1}{2} \dot{\mathrm{m}}\left(\mathrm{V}_{3}^{2}-\mathrm{V}_{0}^{2}\right) \\
\dot{\mathrm{m}}=\rho \mathrm{A}_{\text {disk }} \frac{\mathrm{V}_{0}+\mathrm{V}_{3}}{2}
\end{gathered}
$$

Dimensionless Quantities:

$$
\begin{gathered}
\eta_{\text {prop }}=\frac{2}{\frac{\mathrm{V}_{3}}{\mathrm{~V}_{0}}+1} \\
\mathrm{C}_{\mathrm{P}}=\frac{1}{2}\left(\frac{\mathrm{V}_{3}}{\mathrm{~V}_{0}}+1\right)\left(\left(\frac{\mathrm{V}_{3}}{\mathrm{~V}_{0}}\right)^{2}-1\right) \\
\mathrm{C}_{\mathrm{T}}=\left(\left(\frac{\mathrm{V}_{3}}{\mathrm{~V}_{0}}\right)^{2}-1\right) \\
\frac{\dot{\mathrm{m}}}{\rho A_{\mathrm{d} \mathrm{sk}} \mathrm{V}_{0}}=\frac{1}{2}\left(1+\frac{\mathrm{V}_{3}}{\mathrm{~V}_{0}}\right)
\end{gathered}
$$

\subsubsection{Incompressible Ducted Propeller}

Likewise, the governing equations derived for the incompressible ducted Actuator Disk Theory are applied to the ducted propeller. The performance parameters of interest are again the power coefficient, the total thrust coefficient, and the propulsive efficiency. The definition of the power and the total thrust coefficient in equations 1-51 and 1-53 also applies to the ducted propeller

$$
\begin{gathered}
\mathrm{C}_{\mathrm{P}}=\frac{\mathrm{P}_{\text {input }}}{\mathrm{P}_{\text {available }}}=\frac{\frac{1}{2} \mathrm{~m}\left(\mathrm{~V}_{3}^{2}-\mathrm{V}_{0}^{2}\right)}{\frac{1}{2} \rho \mathrm{A}_{\text {disk }} \mathrm{V}_{0}^{3}} \\
\mathrm{C}_{\mathrm{T}}=\frac{\mathrm{T}_{\text {total }}}{\mathrm{T}_{\text {dynamic }}}=\frac{\dot{\mathrm{m}}\left(\mathrm{V}_{3}-\mathrm{V}_{0}\right)}{\frac{1}{2} \rho \mathrm{A}_{\text {disk }} \mathrm{V}_{0}^{2}}
\end{gathered}
$$

Substituting $\rho A_{0} V_{0}$ for the mass flowrate and the $A_{0}$ given in equation 1-49, the coefficients can be simplified as follows

$$
\begin{gathered}
C_{P}=\frac{V_{3}}{V_{0}}\left(\left(\frac{V_{3}}{V_{0}}\right)^{2}-1\right) \\
C_{T}=2 \frac{V_{3}}{V_{0}}\left(\frac{V_{3}}{V_{0}}-1\right)
\end{gathered}
$$

Because the equations for the power addition to the flow by the disk and the propulsive power are the same as for the bare propeller, the propulsive efficiency in equation 1-63 also applies to the 
ducted propeller. In summary, the performance parameters for the incompressible ducted propeller are summarized below in terms of the far upstream and downstream velocities.

Dimensional Quantities:

$$
\begin{gathered}
\mathrm{T}_{\text {total }}=\dot{\mathrm{m}}\left(\mathrm{V}_{3}-\mathrm{V}_{0}\right) \\
\mathrm{T}_{\text {disk }}=\frac{1}{2} \rho \mathrm{A}_{\text {disk }}\left(\mathrm{V}_{3}^{2}-\mathrm{V}_{0}^{2}\right) \\
\mathrm{T}_{\text {lip }}=\frac{1}{2} \rho \mathrm{V}_{0}^{2} \mathrm{~A}_{\text {disk }}\left(\frac{\mathrm{V}_{3}}{\mathrm{~V}_{0}}-1\right)^{2} \\
\mathrm{P}_{\text {input }}=\frac{1}{2} \dot{\mathrm{m}}\left(\mathrm{V}_{3}^{2}-\mathrm{V}_{0}^{2}\right) \\
\dot{\mathrm{m}}=\rho \mathrm{A}_{\text {disk }} \mathrm{V}_{3}
\end{gathered}
$$

Dimensionless Quantities:

$$
\begin{gathered}
\eta_{\text {prop }}=\frac{2}{\frac{\mathrm{V}_{3}}{\mathrm{~V}_{0}}+1} \\
\mathrm{C}_{\mathrm{P}}=\frac{\mathrm{V}_{3}}{\mathrm{~V}_{0}}\left(\left(\frac{\mathrm{V}_{3}}{\mathrm{~V}_{0}}\right)^{2}-1\right) \\
\mathrm{C}_{\mathrm{T}}=2 \frac{\mathrm{V}_{3}}{\mathrm{~V}_{0}}\left(\frac{\mathrm{V}_{3}}{\mathrm{~V}_{0}}-1\right) \\
\frac{\dot{\mathrm{m}}}{\rho \mathrm{A}_{\mathrm{d} \mathrm{sk}} \mathrm{V}_{0}}=\frac{\mathrm{V}_{3}}{\mathrm{~V}_{0}}
\end{gathered}
$$

Next, these performance parameters of the ducted and the bare propeller are plotted against the power coefficient for comparison.

\subsubsection{Results and Discussion - Incompressible Bare and Ducted Propeller}

To obtain the solutions to the ducted and bare propeller, the disk area is set to $1 \mathrm{ft}^{2}$, the freestream velocity to $10 \mathrm{ft} / \mathrm{s}$, and the free-stream pressure and density to that of sea level static conditions, which are $2166.8 \mathrm{lbf} / \mathrm{ft}^{2}$ and $2.329 \times 10^{-3}$ slugs $/ \mathrm{ft}^{3}$ respectively. The propulsive efficiency, the pressure rise across the disk, and the total thrust coefficient are compared between the bare and ducted propeller against the power coefficient as shown in Figure 4 to 6 . Note that a power coefficient of zero corresponds to no power addition to the flow at the disk plane, which 
results in the far downstream velocity being equal to the free-stream velocity, giving a propulsive efficiency of one and a total thrust coefficient of zero. In addition, the contribution of the lip thrust and the propeller thrust to the total thrust is shown in Figure 7. Note that the pressure rise across the disk is divided by the dynamic pressure $\frac{1}{2} \rho \mathrm{V}_{0}^{2}$, associated with the free-stream velocity, and the lip and the propeller thrusts by the reference force $\frac{1}{2} \rho V_{0}^{2} A_{\text {disk }}$ to be made into dimensionless quantities.

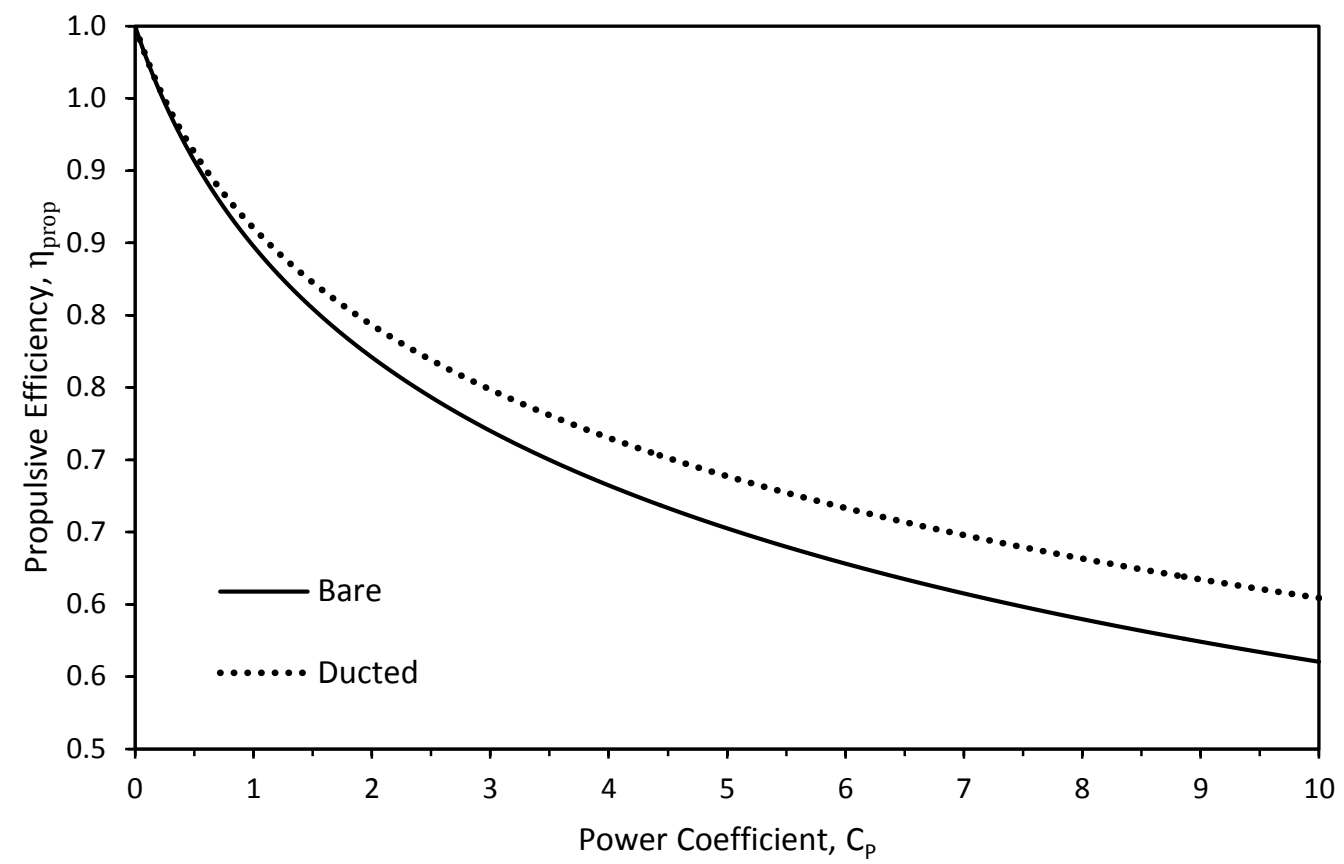

Figure 4. Propulsive efficiency versus the power coefficient between the ducted and bare propeller for incompressible flow. 


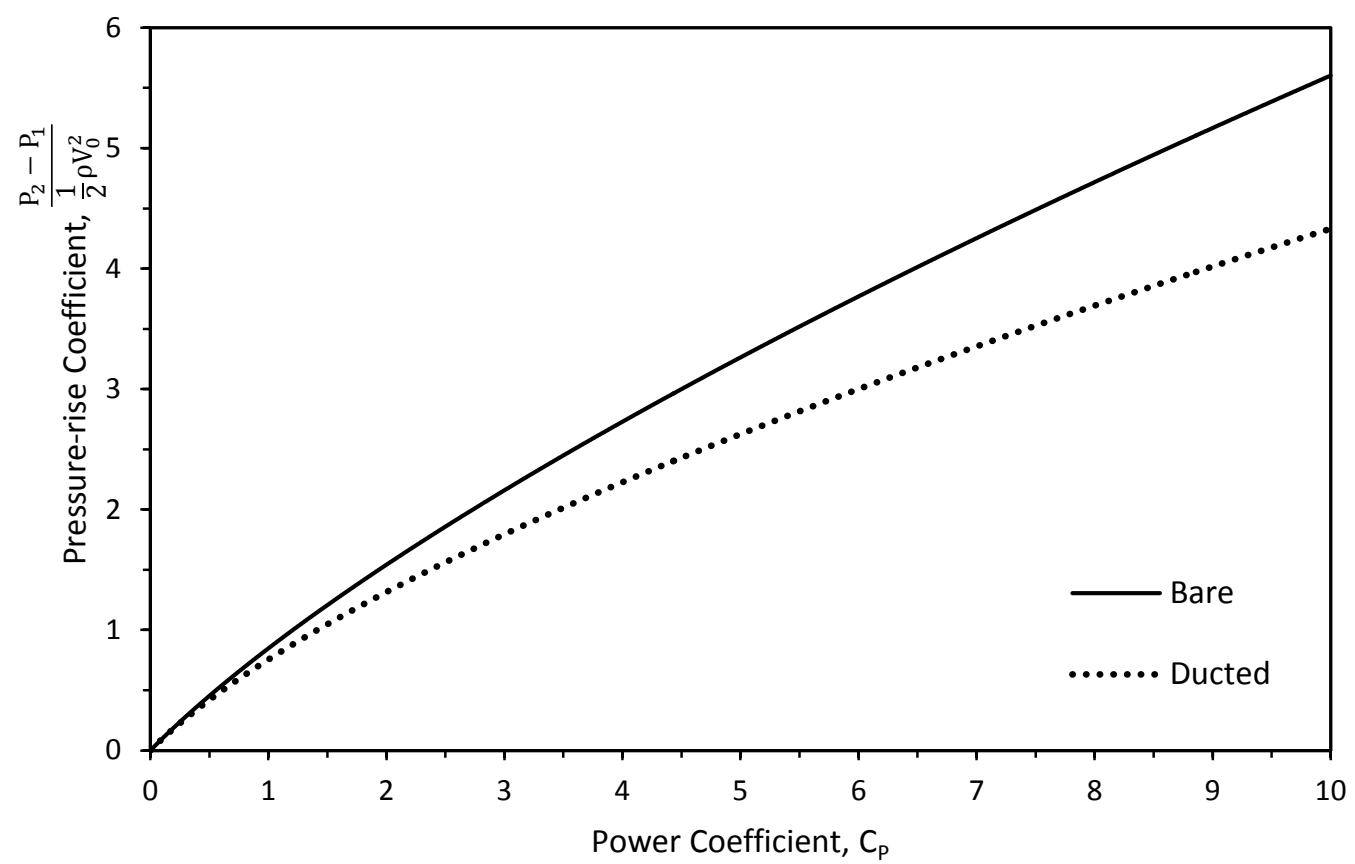

Figure 5. Comparison of the pressure rise across the disk between the bare and ducted propeller for incompressible flow.

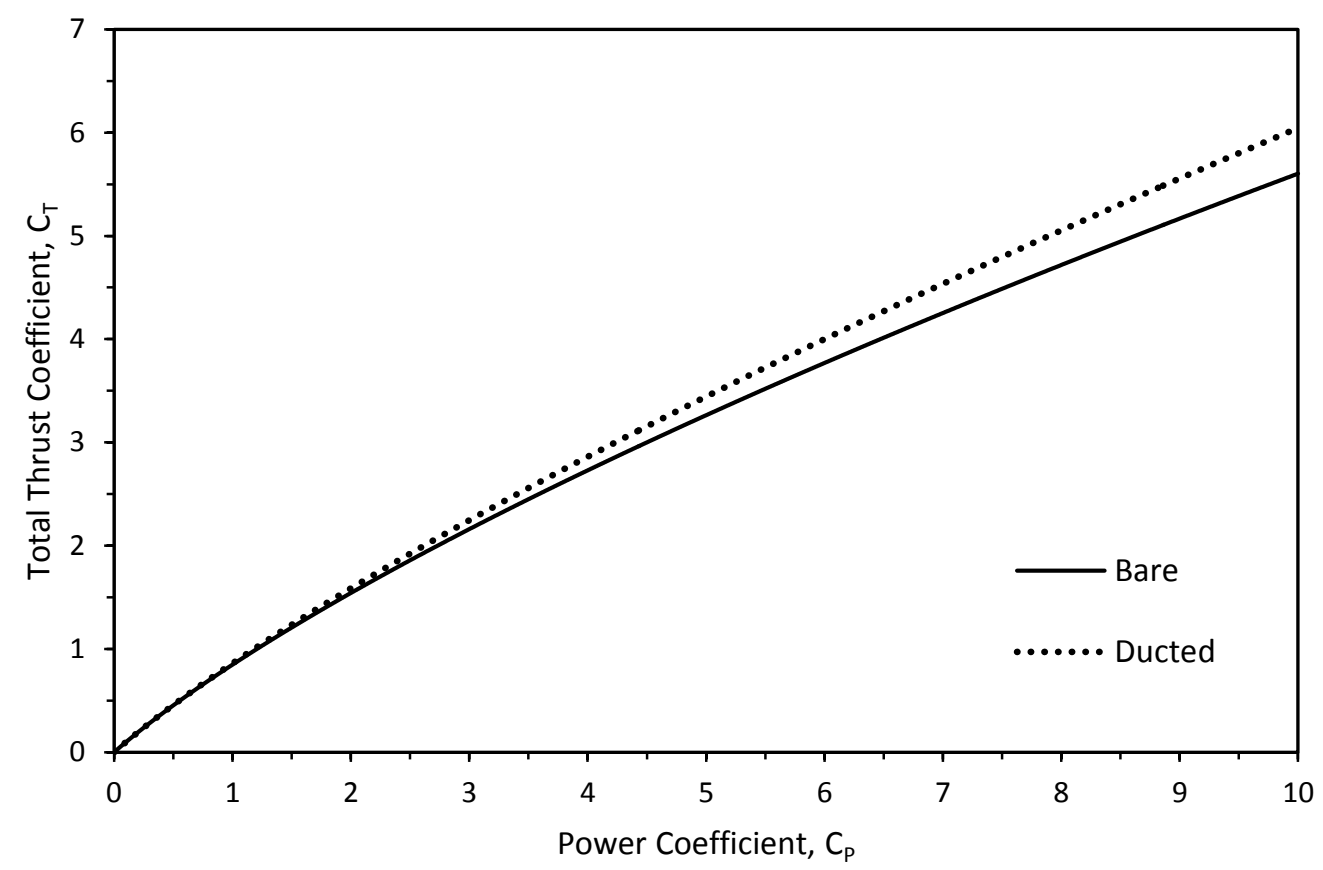

Figure 6. Comparison of the total thrust between the bare and ducted propeller for incompressible flow. 


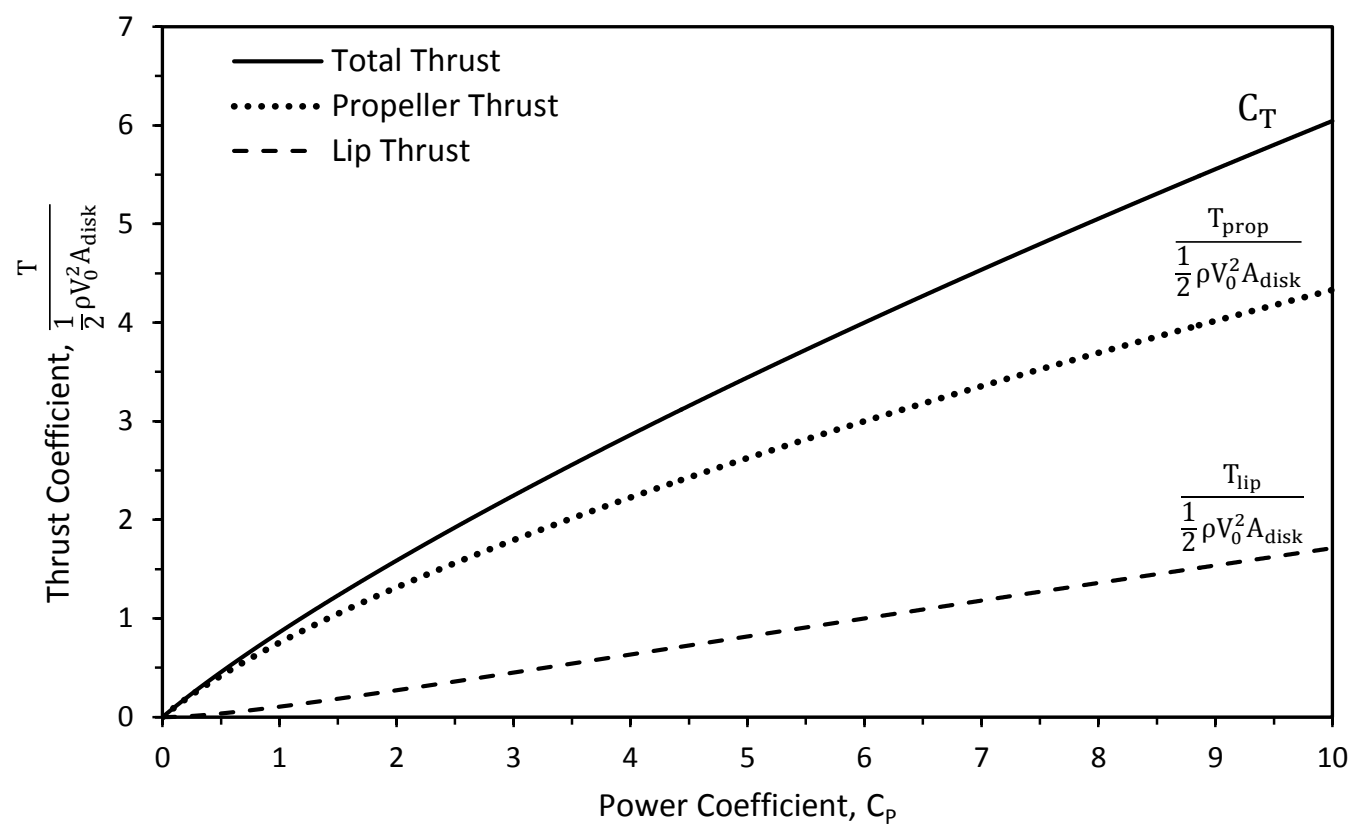

Figure 7. The individual contribution of the propeller thrust and the lip thrust to the total thrust for the incompressible ducted propeller.
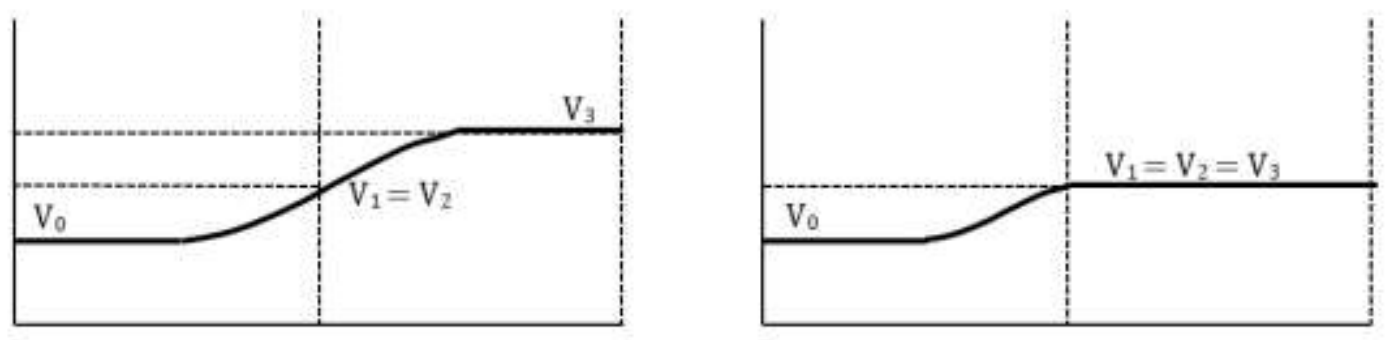

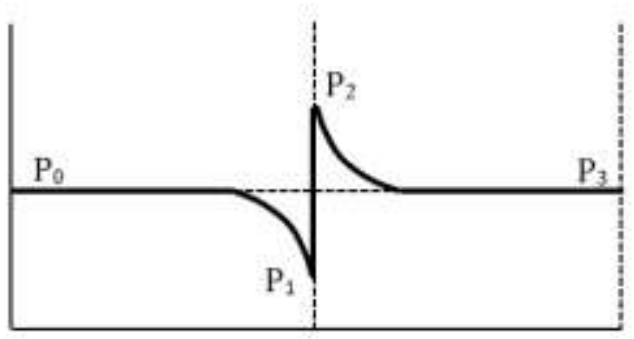

Bare Propeller

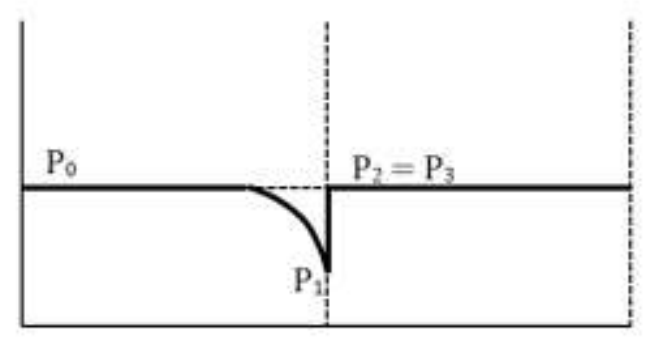

Ducted Propeller

Figure 8. Velocity and pressure across the stations for the bare propeller (left) and the ducted propeller (right).

Figure 4 and Figure 6 shows that the ducted propeller has a higher propulsive efficiency and a total thrust coefficient than the bare propeller, respectively, for all power coefficients. However, the bare propeller has a larger pressure rise across the disk than the ducted propeller as observed 
in Figure 5. When considering the ducted propeller, Figure 7 conveys that the propeller thrust is always greater than the lip thrust and thus contributes more significantly to the total thrust especially at high power coefficients.

An approximation of how the velocity and pressure change throughout the stations for both the ducted and bare propeller are shown in Figure 8. Since power is added to the flow at the disk plane, the far downstream velocity is greater than the value at the free-stream while the velocity at the disk is the average of the two for the bare propeller and is equal to the far downstream velocity for the ducted propeller. Because the mass flowrate throughout the streamtube is constant, the cross-sectional area at the far upstream must be greater than that at the slipstream to account for the lower velocity. It can be observed that the pressure drops from the far upstream to the station immediately before the disk, rises abruptly across the disk, and then drops back to the free-stream pressure. Note that all of these results are consistent with Horlock [1], Glauert [2], and Küchemann and Weber [3] and can be found in most Fluid Mechanics textbooks. The specific case where the free-stream velocity is zero for the bare and ducted propeller is further investigated in Appendix A. Next, the classical Actuator Disk Theory is applied to the bare turbine.

\subsubsection{Incompressible Bare Turbine}

The performance parameters of the bare turbine will be established using the governing equations of the classical bare Actuator Disk Theory. As mentioned previously, a turbine is an energyextracting device that extracts useful energy from the total kinetic energy of the free-flowing fluid and experiences a drag force exerted by the flow. Since the governing equations for the classical theory are derived assuming that the disk is an energy-adding device, a sign change is necessary to the thrust and power equations. Because a drag force is in the opposite direction of the flow, the total drag exerted by the flow is the negative of the total thrust given in equation 1-15

$$
\mathrm{D}_{\text {total }}=-\mathrm{T}_{\text {total }}=\dot{\mathrm{m}}\left(\mathrm{V}_{0}-\mathrm{V}_{3}\right)
$$


The drag force experienced by the turbine is also the negative of the disk thrust given in equation 1-16

$$
\mathrm{D}_{\mathrm{turb}}=-\mathrm{T}_{\text {disk }}=\frac{1}{2} \rho \mathrm{A}_{\text {disk }}\left(\mathrm{V}_{0}^{2}-\mathrm{V}_{3}^{2}\right)
$$

For the bare turbine, the total drag is equal to the turbine drag.

$$
\mathrm{D}_{\text {total }}=\mathrm{D}_{\text {turb }}
$$

The power extracted by the turbine is the negative of the power added by the disk in equation 117 to signify that work is done on the turbine rather than on the flow

$$
\mathrm{P}_{\text {extracted }}=-\mathrm{P}_{\text {disk }}=\frac{1}{2} \dot{\mathrm{m}}\left(\mathrm{V}_{0}^{2}-\mathrm{V}_{3}^{2}\right)
$$

Next, the total drag coefficient, the power extraction efficiency, and the Betz Limit for the bare turbine will be established. The total drag coefficient of the bare turbine is found by dividing the total drag in equation $1-80$ by the reference force to give

$$
C_{D}=\frac{D_{\text {total }}}{T_{\text {dynamic }}}=\frac{\frac{1}{2} \rho A_{\text {disk }}\left(V_{0}^{2}-V_{3}^{2}\right)}{\frac{1}{2} \rho A_{\text {disk }} V_{0}^{2}}
$$

which simplifies to

$$
C_{D}=\left(1-\left(\frac{V_{3}}{V_{0}}\right)^{2}\right)
$$

The figure of merit for a turbine is its power extraction efficiency, which is a ratio of the power extracted by the turbine to the total power available within the free-flowing stream in an area equal to the disk area.

$$
\eta_{\text {turb }}=\frac{P_{\text {extracted }}}{P_{\text {available }}}=\frac{\frac{1}{2} \dot{m}\left(V_{0}^{2}-V_{3}^{2}\right)}{\frac{1}{2} \rho A_{\text {disk }} V_{0}^{3}}
$$

By using the mass flowrate given in 1-18, the power extraction efficiency in 1-86 is a function of the velocity ratio between the far upstream and far downstream stations

$$
\eta_{\text {turb }}=\left(1+\frac{\mathrm{v}_{3}}{\mathrm{v}_{0}}\right)\left(1-\left(\frac{\mathrm{v}_{3}}{\mathrm{v}_{0}}\right)^{2}\right)
$$

The ratio of the far downstream to upstream velocity in equation 1-87 will be defined as $r$

$$
r=\frac{v_{3}}{v_{0}}
$$


Note that this velocity ratio can never exceed 1 because the velocity in the slipstream of the turbine slows down as the turbine captures useful power from the total kinetic energy of the freestream flow. As a result, $V_{3}$ is always less than $V_{0}$ as long as power is being extracted by the turbine. Likewise, $\mathrm{r}$ can never be zero because it is not possible for the turbine to extract $100 \%$ of the total kinetic energy in the free-stream flow for useful power. When $r$ is equal to one, there is no power extraction.

By substituting equation 1-88 into the equation 1-87, the turbine's power extraction efficiency is expressed in terms of $r$

$$
\eta_{\text {turb }}=(1+r)\left(1-r^{2}\right)
$$

Since the power extraction efficiency is in terms of only one independent variable, the maximum power extraction efficiency can be determined by taking the derivative of equation 1-89 with respect to $r$ and setting it equal to zero. As a result, the maximum power extraction efficiency is $16 / 27$, or approximately 0.593 , occurring at a velocity ratio of $1 / 3$. This maximum power extraction efficiency is known as the Betz's limit [7] and signifies the maximum theoretical power that can be extracted by a turbine from the free-flowing fluid. The performance parameters of the incompressible bare turbine are summarized below in terms of the far upstream and downstream velocities

Dimensional Quantities:

$$
\begin{gathered}
\mathrm{D}_{\text {total }}=\dot{\mathrm{m}}\left(\mathrm{V}_{0}-\mathrm{V}_{3}\right) \\
\mathrm{D}_{\text {turb }}=\frac{1}{2} \rho \mathrm{A}_{\text {disk }}\left(\mathrm{V}_{0}^{2}-\mathrm{V}_{3}^{2}\right) \\
\mathrm{P}_{\text {extracted }}=\frac{1}{2} \dot{\mathrm{m}}\left(\mathrm{V}_{0}^{2}-\mathrm{V}_{3}^{2}\right) \\
\dot{\mathrm{m}}=\rho \mathrm{A}_{\text {disk }} \frac{\mathrm{V}_{0}+\mathrm{V}_{3}}{2}
\end{gathered}
$$

Dimensionless Quantities: 


$$
\begin{gathered}
\eta_{\text {turb }}=\left(1+\frac{V_{3}}{V_{0}}\right)\left(1-\left(\frac{V_{3}}{V_{0}}\right)^{2}\right) \\
C_{D}=\left(1-\left(\frac{V_{3}}{V_{0}}\right)^{2}\right) \\
\frac{\dot{m}}{\rho A_{d 1 s k} V_{0}}=\frac{1}{2}\left(1+\frac{V_{3}}{V_{0}}\right)
\end{gathered}
$$

\subsubsection{Incompressible Ducted Turbine}

Likewise, the governing equations of the incompressible ducted Actuator Disk Theory are applied to the ducted turbine. Note that the total and turbine drag and the power extraction from the flow given in equations $1-80,1-81$, and 1-83 also applies to the ducted turbine. However, the total drag is not equal to the turbine drag; it is the difference between the drag on the turbine and the lip thrust.

$$
\mathrm{D}_{\text {total }}=\mathrm{D}_{\text {turb }}-\mathrm{T}_{\text {lip }}
$$

The definition of the total drag coefficient and the power extraction efficiency given in equation 1-84 and 1-86 also applies to the ducted turbine

$$
\begin{gathered}
C_{D}=\frac{D_{\text {total }}}{T_{\text {dynamic }}}=\frac{\dot{\mathrm{m}}\left(\mathrm{V}_{0}-\mathrm{V}_{3}\right)}{\frac{1}{2} \rho \mathrm{A}_{\text {disk }} \mathrm{V}_{0}^{2}} \\
\eta_{\text {turb }}=\frac{\mathrm{P}_{\text {extracted }}}{\mathrm{P}_{\text {available }}}=\frac{\frac{1}{2} \dot{\mathrm{m}}\left(\mathrm{V}_{0}^{2}-\mathrm{V}_{3}^{2}\right)}{\frac{1}{2} \rho \mathrm{A}_{\text {disk }} \mathrm{V}_{0}^{3}}
\end{gathered}
$$

Substituting $\rho \mathrm{A}_{0} \mathrm{~V}_{0}$ for the mass flowrate and the $\mathrm{A}_{0}$ given in equation 1-49, the total drag coefficient and the power extraction efficiency can be simplified as follows

$$
\begin{gathered}
C_{D}=2 \frac{\mathrm{V}_{3}}{\mathrm{~V}_{0}}\left(1-\frac{\mathrm{V}_{3}}{\mathrm{~V}_{0}}\right) \\
\eta_{\text {turb }}=\frac{\mathrm{V}_{3}}{\mathrm{~V}_{0}}\left(1-\left(\frac{\mathrm{V}_{3}}{\mathrm{~V}_{0}}\right)^{2}\right)
\end{gathered}
$$

To determine the Betz limit for the ducted turbine, the same analysis as for the bare turbine is done. Asserting that $r=\frac{V_{3}}{V_{0}}$, the power extraction efficiency can be put in terms of $r$

$$
\mathrm{C}_{\mathrm{p}}=\mathrm{r}\left(1-\mathrm{r}^{2}\right)
$$


By differentiating equation 1-102 with respect to $r$ and setting it equal to zero, the Betz limit, or the maximum power extraction efficiency, for the ducted turbine is $2 / 3^{3 / 2}$ or approximately 0.385 when the far down-to-upstream velocity ratio is roughly 0.577 . This shows that the Betz limit for the ducted turbine is 0.208 lower and occurs at velocity ratio that is 0.052 higher than the bare turbine. The performance parameters of the incompressible ducted turbine are summarized below in terms of the far upstream and downstream velocities

Dimensional Quantities:

$$
\begin{gathered}
\mathrm{D}_{\text {total }}=\dot{\mathrm{m}}\left(\mathrm{V}_{0}-\mathrm{V}_{3}\right) \\
\mathrm{D}_{\text {turb }}=\frac{1}{2} \rho \mathrm{A}_{\text {disk }}\left(\mathrm{V}_{0}^{2}-\mathrm{V}_{3}^{2}\right) \\
\mathrm{T}_{\text {lip }}=\frac{1}{2} \rho \mathrm{A}_{\text {disk }} \mathrm{V}_{0}^{2}\left(\frac{\mathrm{V}_{3}}{\mathrm{~V}_{0}}-1\right)^{2} \\
\mathrm{P}_{\text {extracted }}=\frac{1}{2} \dot{\mathrm{m}}\left(\mathrm{V}_{0}^{2}-\mathrm{V}_{3}^{2}\right) \\
\dot{\mathrm{m}}=\rho \mathrm{A}_{\text {disk }} \frac{\mathrm{V}_{0}+\mathrm{V}_{3}}{2}
\end{gathered}
$$

Dimensionless Quantities:

$$
\begin{gathered}
\eta_{\text {turb }}=\frac{\mathrm{V}_{3}}{\mathrm{~V}_{0}}\left(1-\left(\frac{\mathrm{V}_{3}}{\mathrm{~V}_{0}}\right)^{2}\right) \\
\mathrm{C}_{\mathrm{D}}=2 \frac{\mathrm{V}_{3}}{\mathrm{~V}_{0}}\left(1-\frac{\mathrm{V}_{3}}{\mathrm{~V}_{0}}\right) \\
\frac{\dot{\mathrm{m}}}{\rho \mathrm{A}_{\mathrm{d} \mathrm{sk}} \mathrm{V}_{0}}=\frac{\mathrm{V}_{3}}{\mathrm{~V}_{0}}
\end{gathered}
$$

These performance parameters of both the ducted and the bare turbine are plotted against the velocity ratio $r$ for comparison.

\subsubsection{Results and Discussion - Incompressible Bare and Ducted Turbine}

The solutions are determined for the same disk area and free-stream flow conditions as those of the bare and ducted propeller. The power extraction efficiency, the pressure drop across the disk, and the total drag coefficient are compared between the bare and the ducted turbine in Figure 9 to 
Figure 11 in terms of the velocity ratio $r$. The individual contribution of the turbine drag and the lip thrust to the total drag is shown for the ducted turbine in Figure 12. Note that the pressure drop, the lip thrust, and the turbine drag are made dimensionless in the same way as were done for the propeller case.

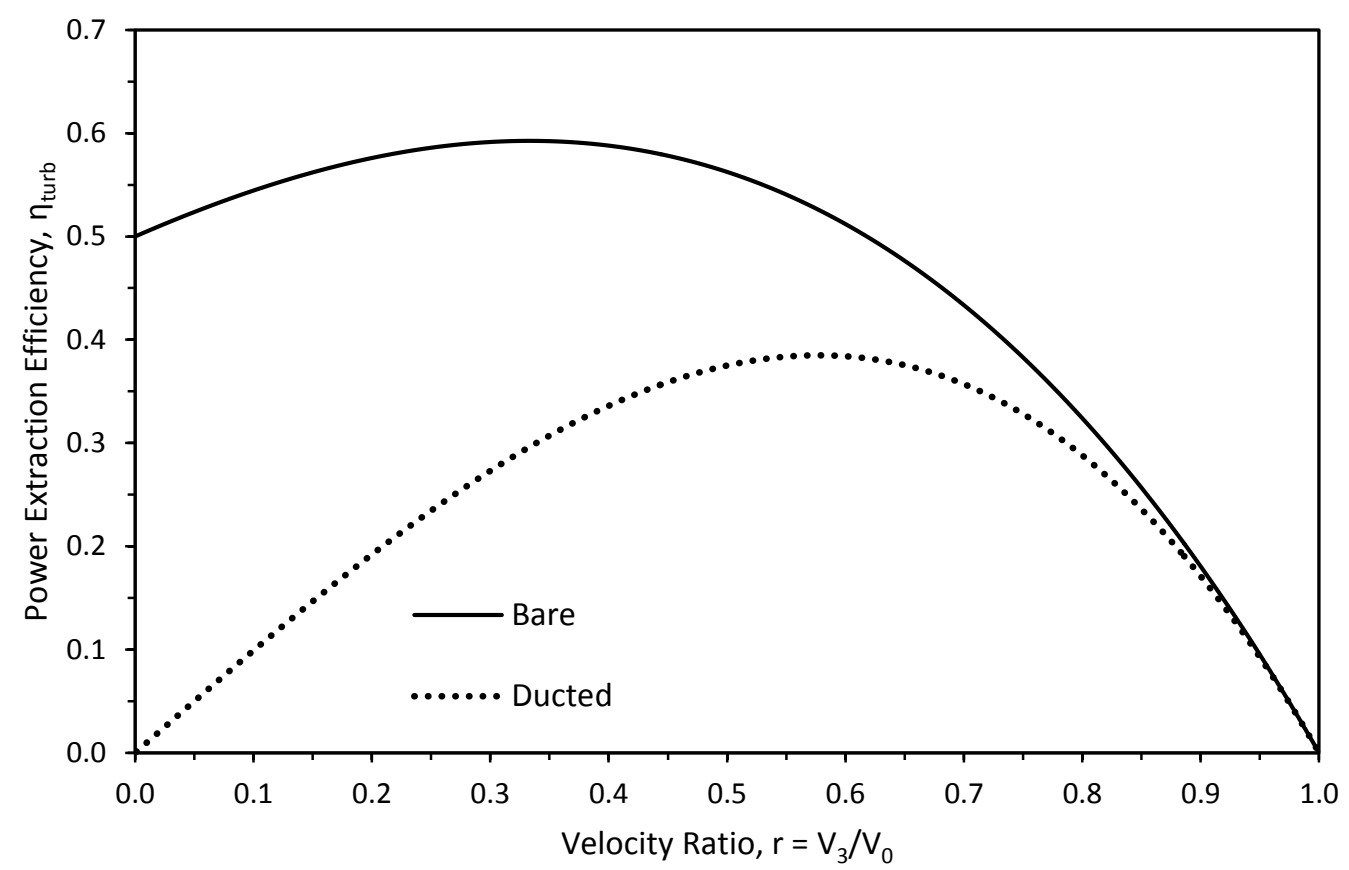

Figure 9. Comparison of the power extraction efficiency and the Betz limit between the bare and ducted turbine for incompressible flow. 


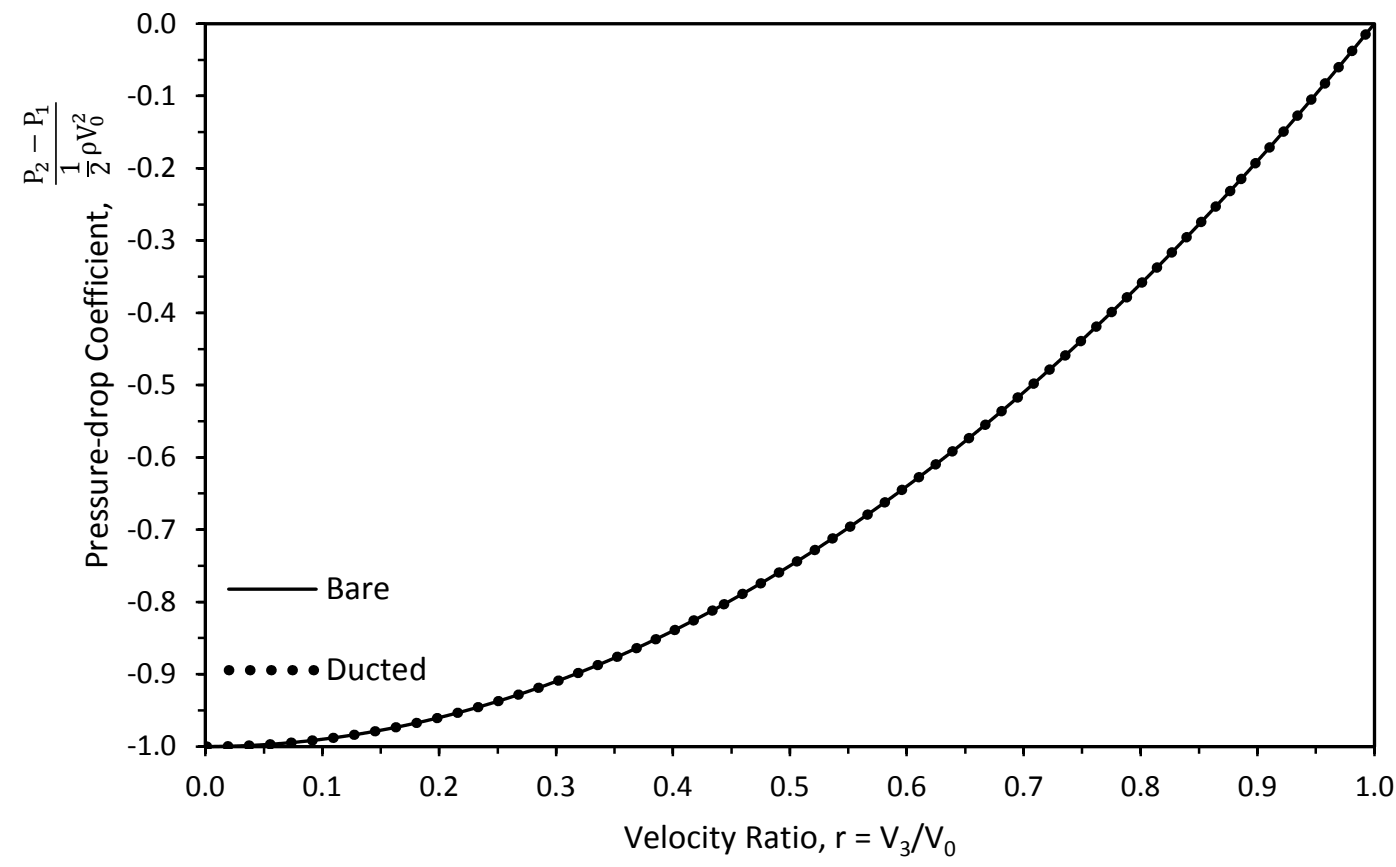

Figure 10. Comparison of the pressure drop across the disk between the bare and ducted turbine for incompressible flow.

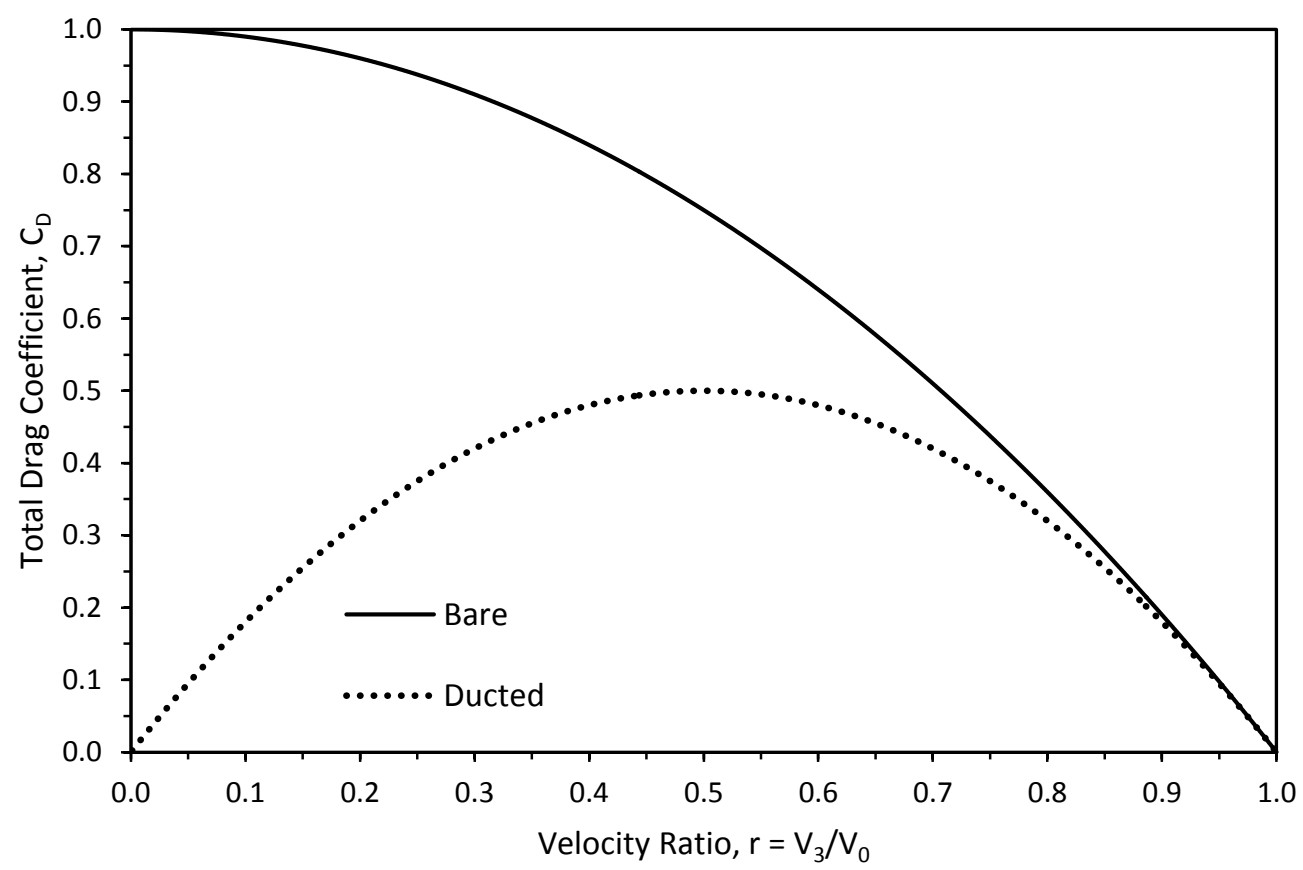

Figure 11. Comparison of the total drag between the bare and ducted turbine for incompressible flow. 


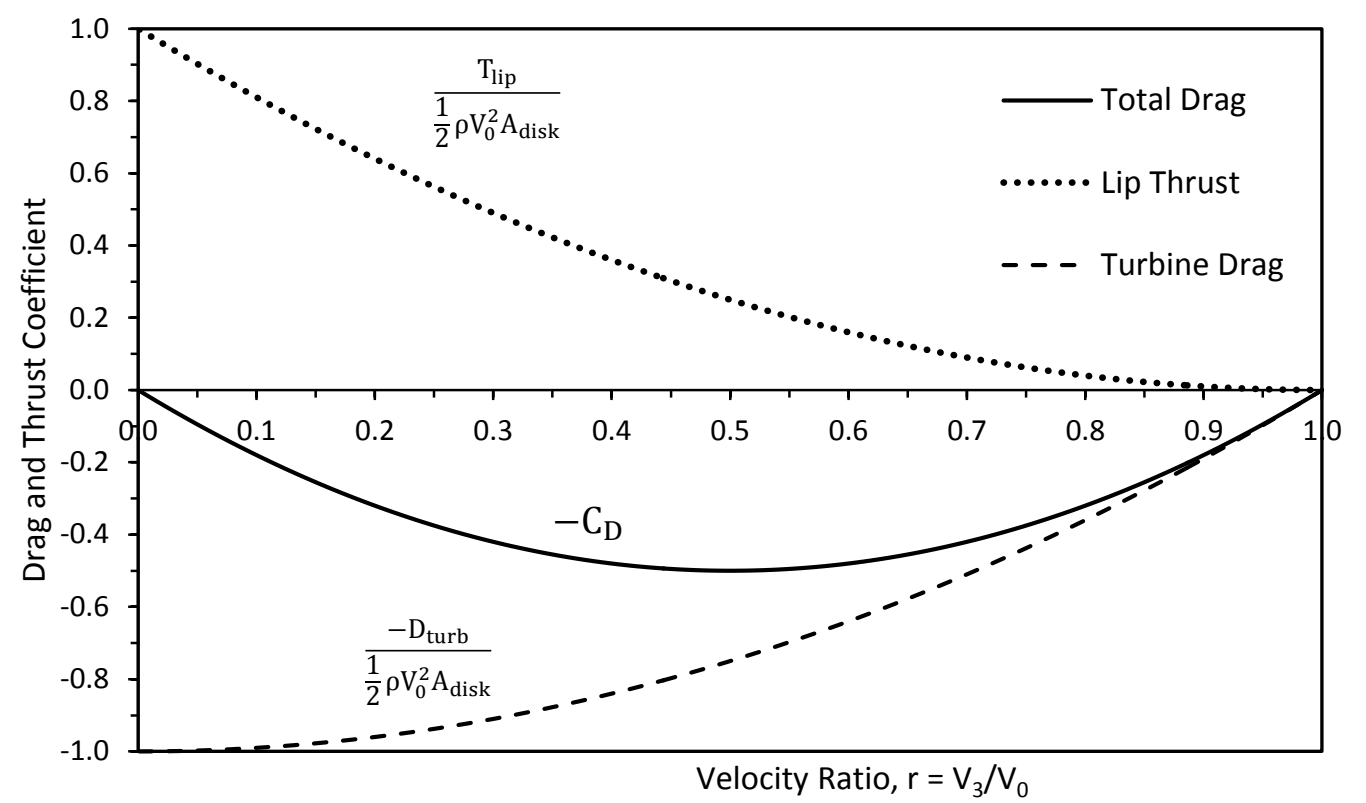

Figure 12. The individual contribution of the turbine drag and the lip thrust to the total drag for the incompressible ducted turbine. Note that the total drag and the turbine drag are made negative to signify that they are in the opposite direction of the flow.
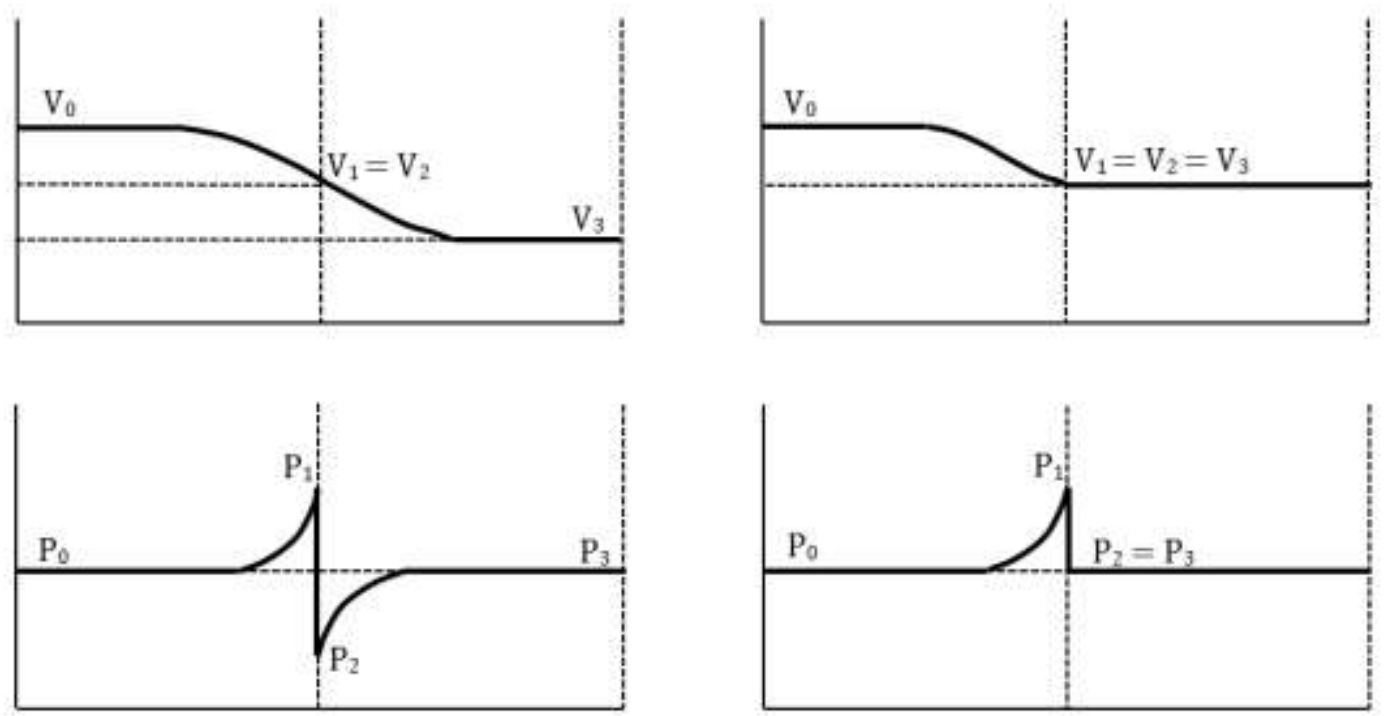

Bare Turbine

Ducted Turbine

Figure 13. Velocity and pressure throughout the stations for the bare turbine (left) and for the ducted turbine (right). 
Unlike the propeller, Figure 9 shows that the bare turbine has a higher power extraction efficiency than the ducted turbine for all velocity ratios $r$. Thus, the bare turbine has a higher Betz limit and occurs at a lower velocity ratio $r$ than the ducted turbine. Likewise, the bare turbine has a greater total drag coefficient than the ducted turbine as observed in Figure 11; while, the pressure drop across the disk is the same for both cases as shown in Figure 10. Figure 12 shows that the magnitude of the turbine drag is greater than that of the lip thrust and thus contributes more greatly to the total drag.

An approximation of the velocity and the pressure throughout the stations are given in Figure 13. Since energy is being extracted by the turbine at the disk plane, the far downstream velocity is lower than that at the free-stream; while the velocity at the disk is again the average of the two velocities for the bare turbine and is the same as the far downstream velocity for the ducted turbine. This means that the free-stream area is smaller than that at the far downstream to account for the higher free-stream velocity since the density and the mass flowrate throughout the streamtube are constant. Lastly, the pressure increases from the free-stream to immediately before the disk, drops discontinuously across the disk, and increases to the free-stream pressure for the bare turbine and drops to the free-stream pressure for the ducted turbine. Again, these results agree with Horlock [1], Glauert [2], and Küchemann and Weber [3] and can be found in many Fluids Mechanics textbooks. Next, the method of solution will be briefly discussed. 


\subsection{Method of Solution to the Classical Actuator Disk Theory}

Although the performance of the propeller and the turbine has been determined and discussed, the method to obtaining the performance parameters will be briefly established. This is mainly done to later support the reasoning behind the method of solution for the compressible Actuator Disk Theory-a topic that will be touched upon in Chapters 2 to 4.

The governing equations of the classical Actuator Disk Theory provide a system of equations necessary to perform a complete analysis of the flow and the actuator disk. A complete analysis is one where the performance parameters of the disk are determined and all of the flow variables such as the density, pressure, velocity, and the streamtube cross-sectional area are known at each of the four stations. Since the governing equations are a set of non-linear algebraic equation ns, the desired solutions can be determined if there is an equal number of unknowns and equations. The desired solutions, which are the performance parameters of the disk and the flow variables at each station, are determined in terms of a few selected inputs. Usually, the inputs are the disk area, the far downstream velocity, and the free-stream density, pressure, and velocity.

Consider the application of the bare turbine in section 1.3.4. With the five inputs, which are $\rho, \mathrm{P}_{0}, \mathrm{~V}_{0}, \mathrm{~A}_{\text {disk }}$, and $\mathrm{V}_{3}$, the unknown variables left to determine are the following flow variables $A_{0}, P_{1}, V_{1}, P_{2}$, and $A_{3}$ and the following performance parameters of the turbine $D_{\text {total }}, D_{\text {turb }}, P_{\text {extracted }}, \dot{m}, C_{D}$, and $\eta_{\text {turb }}$. These eleven unknown variables can be solved explicitly using the eleven governing equations from 1-19 to 1-23 and 1-90 to 1-95. The same can be done for other applications of the classical Actuator Disk Theory.

In addition, the unknown variables in these equations can easily be isolated and expressed solely in terms of the inputs. They can also easily be made dimensionaless by the given parameters $\rho, \mathrm{P}_{0}, \mathrm{~V}_{0}, \mathrm{~V}_{3}$, and $\mathrm{A}_{\text {disk }}$.

In concluding remarks, the governing equations for the classical Actuator Disk Theory provides the means to solve for the flow variables at each station along with the performance parameters of the actuator disk given the free-stream flow conditions, the far downstream velocity, and the disk 
area. The solutions to the governing equations can easily be determined explicitly, separated and be put solely in terms of the inputs, and be made into dimensionless quantities. It will be shown in later chapters that these characteristics are not inherent in the governing equations for the compressible Actuator Disk Theory. 


\section{COMPRESSIBLE ACTUATOR DISK THEORY}

\subsection{Introduction}

Since compressibility effects arise in real applications of energy-adding or energy-extracting devices, the Actuator Disk Theory will be extended to the regime of compressible subsonic flow, which will be referred to as the compressible Actuator Disk Theory or the compressible theory throughout the current work. The motivation for the current work is to develop a set of governing equations necessary to predict the ideal performance of the microRAT [4] in compressible flow operating at high subsonic Mach numbers, in an effort to prevent sensor dropout of the Boundary Layer Data Systems (BLDS) as discussed in Chapter 1. The governing equations for the compressible Actuator Disk Theory will be established in this chapter for the bare (unducted) and the ducted cases using isentropic thermodynamics and the three conservation laws of mass, momentum, and energy. These governing equations provide a system of equations that is necessary to solve for the flow variables at each station as shown in Figure 14 and to determine the ideal performance of the actuator disk in terms of some input variables. The mechanics of determining the solutions will be discussed in the Method of Solutions. Note that the solutions to the compressible Actuator Disk Theory and its applications are obtained only for subsonic flow, which is the scope of the current thesis.

The compressible theory will be derived for the same assumptions that were made for the classical theory under the List of Assumptions in section 1.2.1, with the exception of the first two as they only apply to incompressible flow. Additional assumptions made specifically for the compressible theory are listed below. The compressible theory differs from the classical theory in ways that the density now varies throughout the streamtube and the Mach number is thus included as a flow variable at each station as shown in Figure 14. In addition to the pressure discontinuity across the disk as observed in the classical theory, the density and velocity are also discontinuous across the disk for compressible flow. Using these 
assumptions, the governing equations for the compressible Actuator Disk Theory will be developed beginning with the bare actuator disk in the next section.

Additional Assumptions for Compressible Flow

10) The flow is compressible and isentropic.

11) The total pressure is constant between stations 0 and 1 and stations 2 and 3 .

12) The velocity, pressure, and density across the actuator disk are discontinuous.

13) The Mach number at any station never exceeds 1 because the scope of the thesis at hand is to observe the compressibility effects on the Actuator Disk Theory only in the subsonic flow regime.

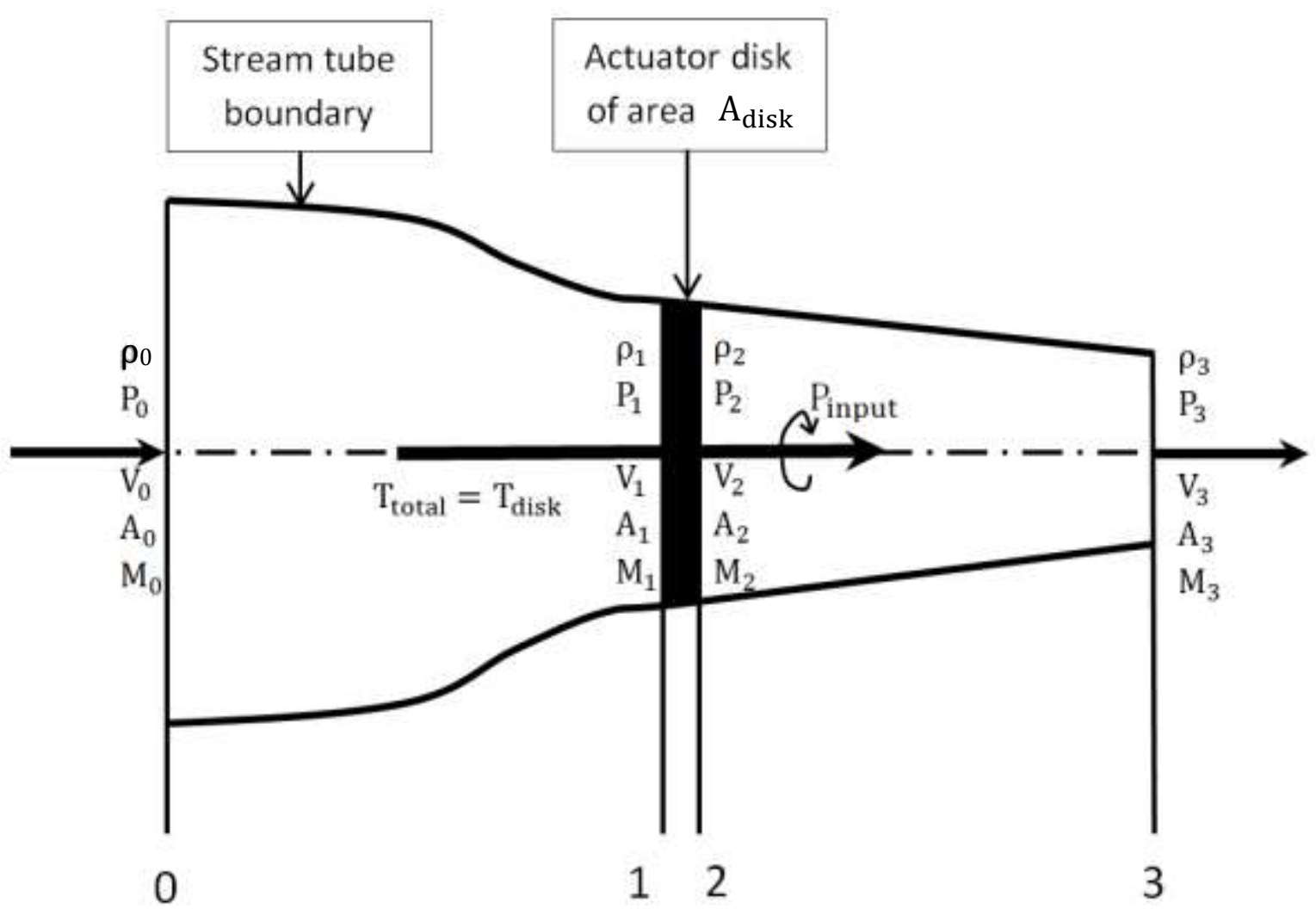

Figure 14. Schematic of the bare, compressible Actuator Disk Theory. 


\subsection{Compressible, Bare Actuator Disk Theory}

The governing equations of the compressible Actuator Disk Theory will be developed for the unducted (bare) actuator disk that is contained within the streamtube boundary as shown in Figure 14. Stations 0 and 3 are the far upstream and downstream where the free-stream pressure exists, while stations 1 and 2 are immediately before and after the actuator disk. The control volume 0-3 contains the flow from station 0 to 3 , the control volumes $0-1$ and 2-3 encloses the flow upstream and downstream of the disk, and lastly the control volume 1-2 contains the flow through the disk plane. Note that the station designations and the control volumes are the same as those for the classical theory. The actuator disk is treated as an energy-adding device where it adds power to the flow at the disk plane and exerts a thrust force on the flow in the direction of the flow.

Since isentropic flow is assumed, the isentropic flow relations are heavily involved in the derivation of the governing equations. The details of the relations will not be discussed but can be found in the NACA report titled "Equations, Tables, and Charts for Compressible Flow" [8].

To begin, the far upstream and downstream densities are shown to be equal by asserting that $\mathrm{P}_{0}=\mathrm{P}_{3}$ into the isentropic pressure-density relation

$$
\frac{\rho_{3}}{\rho_{0}}=\left(\frac{\mathrm{P}_{3}}{\mathrm{P}_{0}}\right)^{\frac{1}{\gamma}}=1
$$

to give

$$
\rho_{0}=\rho_{3}
$$

Next, the flow variables at station 1 are found in terms of the free-stream flow properties. Asserting that $\mathrm{P}_{\mathrm{t}_{0}}=\mathrm{P}_{\mathrm{t}_{1}}$ into the isentropic pressure ratios as shown and combining,

$$
\begin{aligned}
& \frac{\mathrm{P}_{\mathrm{t}_{0}}}{\mathrm{P}_{0}}=\left(1+\frac{\gamma-1}{2} \mathrm{M}_{0}^{2}\right)^{\frac{\gamma}{\gamma-1}} \\
& \frac{\mathrm{P}_{\mathrm{t}_{1}}}{\mathrm{P}_{1}}=\left(1+\frac{\gamma-1}{2} \mathrm{M}_{1}^{2}\right)^{\frac{\gamma}{\gamma-1}}
\end{aligned}
$$

the static pressure immediately before the disk $\mathrm{P}_{1}$ is given as a function of the Mach number at stations 0 and 1 


$$
\frac{\mathrm{P}_{1}}{\mathrm{P}_{0}}=\left(\frac{1+\frac{\gamma-1}{2} \mathrm{M}_{0}^{2}}{1+\frac{\gamma-1}{2} \mathrm{M}_{1}^{2}}\right)^{\frac{\gamma}{\gamma-1}}
$$

A ratio of the cross-sectional area at station 0 to that at station 1 can be established using the isentropic flow relation between area and Mach number and noting that $A_{1}=A_{\text {disk }}$

$$
\frac{A_{0}}{A_{\text {disk }}}=\frac{M_{1}}{M_{0}}\left(\frac{1+\frac{\gamma-1}{2} M_{0}^{2}}{1+\frac{\gamma-1}{2} M_{1}^{2}}\right)^{\frac{\gamma+1}{2(\gamma-1)}}
$$

Next, the density at station 1 is obtained through the isentropic pressure-density relation

$$
\frac{\rho_{1}}{\rho_{0}}=\left(\frac{\mathrm{P}_{1}}{\mathrm{P}_{0}}\right)^{\frac{1}{\gamma}}
$$

The velocity at station 1 can be found using the conservation of mass on the control volume $0-1$.

$$
\frac{\mathrm{V}_{1}}{\mathrm{~V}_{0}}=\frac{\rho_{0}}{\rho_{1}} \frac{\mathrm{A}_{0}}{\mathrm{~A}_{\mathrm{disk}}}
$$

Next, the flow variables at station 3 are related to those at station 0 . By applying the conservation of energy on the control volume $0-3$, it can be observed that the total energy at station $3 E_{3}$ is the sum of the total energy at station $0 \mathrm{E}_{0}$ and the energy added to the flow by the disk. The energy added is denoted as the power addition per unit mass flow across the disk as shown

$$
\mathrm{E}_{0}+\frac{\mathrm{P}_{\text {input }}}{\dot{\mathrm{m}}}=\mathrm{E}_{3}
$$

where $\mathrm{E}_{0}$ and $\mathrm{E}_{3}$ are

$$
\begin{aligned}
& E_{0}=\frac{V_{0}^{2}}{2}+\frac{\gamma}{\gamma-1} \cdot \frac{P_{0}}{\rho_{0}} \\
& E_{3}=\frac{V_{3}^{2}}{2}+\frac{\gamma}{\gamma-1} \cdot \frac{P_{3}}{\rho_{3}}
\end{aligned}
$$

By substitution, the energy equation in equation 2-9 can be expanded as follows

$$
\frac{\mathrm{V}_{0}^{2}}{2}+\frac{\gamma}{\gamma-1} \cdot \frac{\mathrm{P}_{0}}{\rho_{0}}+\frac{\mathrm{P}_{\text {input }}}{\dot{\mathrm{m}}}=\frac{\mathrm{V}_{3}^{2}}{2}+\frac{\gamma}{\gamma-1} \cdot \frac{\mathrm{P}_{3}}{\rho_{3}}
$$

By rearranging and noting that $\mathrm{P}_{3}=\mathrm{P}_{0}$, the total power added by the disk per unit mass flow is the sum of two terms

$$
\frac{\mathrm{P}_{\text {input }}}{\dot{\mathrm{m}}}=\frac{\mathrm{V}_{3}^{2}-\mathrm{V}_{0}^{2}}{2}+\frac{\gamma}{\gamma-1} \frac{\mathrm{P}_{0}}{\rho_{0}}\left(\frac{\rho_{0}}{\rho_{3}}-1\right)
$$


The second term on the right of equation 2-19 goes to zero by asserting that $\rho_{0}=\rho_{3}$, thus the total power added by the disk is

$$
\mathrm{P}_{\text {input }}=\dot{\mathrm{m}} \frac{\mathrm{V}_{3}^{2}-\mathrm{V}_{0}^{2}}{2}
$$

Observe that this is identical to the incompressible power equation in 1-17. From the power equation, the far downstream velocity $V_{3}$ can be determined for a given disk power. Next, $\mathrm{M}_{3}$ can be found by applying the isentropic speed of sound relation

$$
\mathrm{a}_{3}=\sqrt{\gamma \cdot \frac{\mathrm{P}_{3}}{\rho_{3}}}=\sqrt{\gamma \cdot \frac{\mathrm{P}_{0}}{\rho_{0}}}
$$

to the definition of Mach number

$$
\mathrm{M}_{3}=\frac{\mathrm{V}_{3}}{\mathrm{a}_{3}}
$$

to give

$$
\mathrm{M}_{3}=\mathrm{V}_{3}\left(\gamma \cdot \frac{\mathrm{P}_{0}}{\rho_{0}}\right)^{-\frac{1}{2}}
$$

Likewise, the area at the far downstream $\mathrm{A}_{3}$ can be determined using the conservation of mass applied to the control volume $0-3$ along with $\rho_{0}=\rho_{3}$

$$
\frac{A_{3}}{A_{0}}=\frac{\rho_{0}}{\rho_{3}} \cdot \frac{V_{0}}{V_{3}}=\frac{V_{0}}{V_{3}}
$$

Next, the flow variables at station 2 will be determined related to those at station 3 .

The Mach number immediately after the disk $\mathrm{M}_{2}$ is obtained using the isentropic relation between area and Mach number between station 2 and 3

$$
\frac{A_{3}}{A_{\text {disk }}}=\frac{M_{2}}{M_{3}}\left(\frac{1+\frac{\gamma-1}{2} M_{3}^{2}}{1+\frac{\gamma-1}{2} M_{2}^{2}}\right)^{\frac{\gamma+1}{2(\gamma-1)}}
$$

Next, the pressure immediately after the disk $P_{2}$ is obtained by applying that $P_{t_{2}}=P_{t_{3}}$ into the isentropic pressure ratios at stations 2 and 3

$$
\frac{\mathrm{P}_{\mathrm{t}_{2}}}{\mathrm{P}_{2}}=\left(1+\frac{\gamma-1}{2} \mathrm{M}_{2}^{2}\right)^{\frac{\gamma}{\gamma-1}}
$$




$$
\frac{\mathrm{P}_{\mathrm{t}_{3}}}{\mathrm{P}_{3}}=\frac{\mathrm{P}_{\mathrm{t}_{3}}}{\mathrm{P}_{0}}=\left(1+\frac{\gamma-1}{2} \mathrm{M}_{3}^{2}\right)^{\frac{\gamma}{\gamma-1}}
$$

to give

$$
\frac{\mathrm{P}_{2}}{\mathrm{P}_{0}}=\left(\frac{1+\frac{\gamma-1}{2} \mathrm{M}_{3}^{2}}{1+\frac{\gamma-1}{2} \mathrm{M}_{2}^{2}}\right)^{\frac{\gamma}{\gamma-1}}
$$

Again, the density at station 2 is found by the isentropic pressure-density relation

$$
\frac{\rho_{2}}{\rho_{3}}=\frac{\rho_{2}}{\rho_{0}}=\left(\frac{\mathrm{P}_{2}}{\mathrm{P}_{3}}\right)^{\frac{1}{\gamma}}
$$

The velocity at station 2 is found by using the conservation of mass on the control volume 1-2.

$$
\mathrm{V}_{2}=\frac{\rho_{1}}{\rho_{2}} \mathrm{~V}_{1}
$$

By applying the conservation of linear momentum to the control volume $0-3$, the total thrust is found

$$
\mathrm{T}_{\text {total }}=\dot{\mathrm{m}}\left(\mathrm{V}_{3}-\mathrm{V}_{0}\right)
$$

Note that this is the same total thrust given in equation 1-15 for incompressible Actuator Disk Theory. The thrust produced by the disk can be determined by applying the conservation of linear momentum to the control volume 1-2

$$
\mathrm{T}_{\text {disk }}=\dot{\mathrm{m}}\left(\mathrm{V}_{2}-\mathrm{V}_{1}\right)+\mathrm{A}_{\text {disk }}\left(\mathrm{P}_{2}-\mathrm{P}_{1}\right)
$$

Lastly, the mass flowrate can be found from

$$
\dot{\mathrm{m}}=\rho_{1} \mathrm{~A}_{\mathrm{disk}} \mathrm{V}_{1}
$$

At this point in the analysis, it is important to note that all the equations derived above for compressible Actuator Disk Theory from equation 2-1 to 2-27 can be applied to any ideal disk that adds power to the flow stream and generates a thrust force, whether the disk is bare or ducted.

Lastly, since there are no other assemblies besides the actuator disk within the flow stream, the total thrust exerted on the flow is then the thrust produced by the disk, which is only true for the bare case. 


$$
\mathrm{T}_{\text {total }}=\mathrm{T}_{\text {disk }}
$$

This concludes the derivation of the governing equations for the compressible bare Actuator Disk Theory, which are summarized in the next section.

\subsubsection{Summary of Governing Equations}

In summary, the governing equations of the compressible bare Actuator Disk Theory relates the flow variables at each station, the mass flowrate, the total thrust, the disk thrust, and the power addition by the disk to the free-stream flow properties, which are $\gamma, \mathrm{P}_{0}, \rho_{0}$, and $\mathrm{V}_{0}$ and the disk area. The governing equations are summarized below:

$$
\begin{gathered}
\mathrm{M}_{0}=\mathrm{V}_{0}\left(\gamma \cdot \frac{\mathrm{P}_{0}}{\rho_{0}}\right)^{-\frac{1}{2}} \\
\frac{\mathrm{A}_{0}}{\mathrm{~A}_{\mathrm{disk}}}=\frac{\mathrm{M}_{1}}{\mathrm{M}_{0}}\left(\frac{1+\frac{\gamma-1}{2} \mathrm{M}_{0}^{2}}{1+\frac{\gamma-1}{2} \mathrm{M}_{1}^{2}}\right)^{\frac{\gamma+1}{2(\gamma-1)}} \\
\frac{\mathrm{P}_{1}}{\mathrm{P}_{0}}=\left(\frac{1+\frac{\gamma-1}{2} \mathrm{M}_{0}^{2}}{1+\frac{\gamma-1}{2} \mathrm{M}_{1}^{2}}\right)^{\frac{\gamma}{\gamma-1}} \\
\frac{\rho_{1}}{\rho_{0}}=\left(\frac{\mathrm{P}_{1}}{\mathrm{P}_{0}}\right)^{\frac{1}{\gamma}} \\
\frac{\mathrm{V}_{1}}{\mathrm{~V}_{0}}=\frac{\rho_{0}}{\rho_{1}} \frac{\mathrm{A}_{0}}{\mathrm{~A}_{\mathrm{disk}}} \\
\frac{\mathrm{M}_{3}}{=}=\mathrm{V}_{3}\left(\gamma \cdot \frac{\mathrm{P}_{0}}{\rho_{0}}\right)^{-\frac{1}{2}} \\
\frac{\mathrm{A}_{3}}{\mathrm{~A}_{0}}=\frac{\mathrm{V}_{0}}{\mathrm{~V}_{3}} \\
\left.\frac{\mathrm{A}_{3}}{\mathrm{~A}_{\mathrm{disk}}}=\frac{\left(\frac{\mathrm{P}_{2}}{\mathrm{P}_{0}}\right)^{\frac{1}{\gamma}}}{\mathrm{M}_{3}}\left(\frac{1+\frac{\gamma-1}{2} \mathrm{M}_{3}^{2}}{1+\frac{\gamma-1}{2} \mathrm{M}_{2}^{2}}\right)^{\frac{\gamma+1}{2(\gamma-1)}} \mathrm{M}_{2}^{2}\right)^{\frac{\gamma}{\gamma-1}} \\
\mathrm{P}^{2}
\end{gathered}
$$




$$
\begin{gathered}
\mathrm{V}_{2}=\frac{\rho_{1}}{\rho_{2}} \mathrm{~V}_{1} \\
\dot{\mathrm{m}}=\rho_{1} \mathrm{~A}_{\text {disk }} \mathrm{V}_{1} \\
\mathrm{~T}_{\text {total }}=\dot{\mathrm{m}}\left(\mathrm{V}_{3}-\mathrm{V}_{0}\right) \\
\mathrm{T}_{\text {disk }}=\dot{\mathrm{m}}\left(\mathrm{V}_{2}-\mathrm{V}_{1}\right)+\mathrm{A}_{\text {disk }}\left(\mathrm{P}_{2}-\mathrm{P}_{1}\right) \\
\mathrm{P}_{\text {input }}=\dot{\mathrm{m}} \frac{\mathrm{V}_{3}^{2}-\mathrm{V}_{0}^{2}}{2} \\
\mathrm{~T}_{\text {total }}=\mathrm{T}_{\text {disk }}
\end{gathered}
$$

In comparison to the classical bare Actuator Disk Theory, the thrust produced by the disk in equation 2-42 now contains an additional term due to the velocity discontinuity across the disk, while the total thrust and the total power addition by the disk in equation 2-41 and 2-43 remains the same as for incompressible flow. In the next section, the governing equations will be considered for the ducted case of the compressible Actuator Disk Theory. 


\subsection{Compressible, Ducted Actuator Disk Theory}

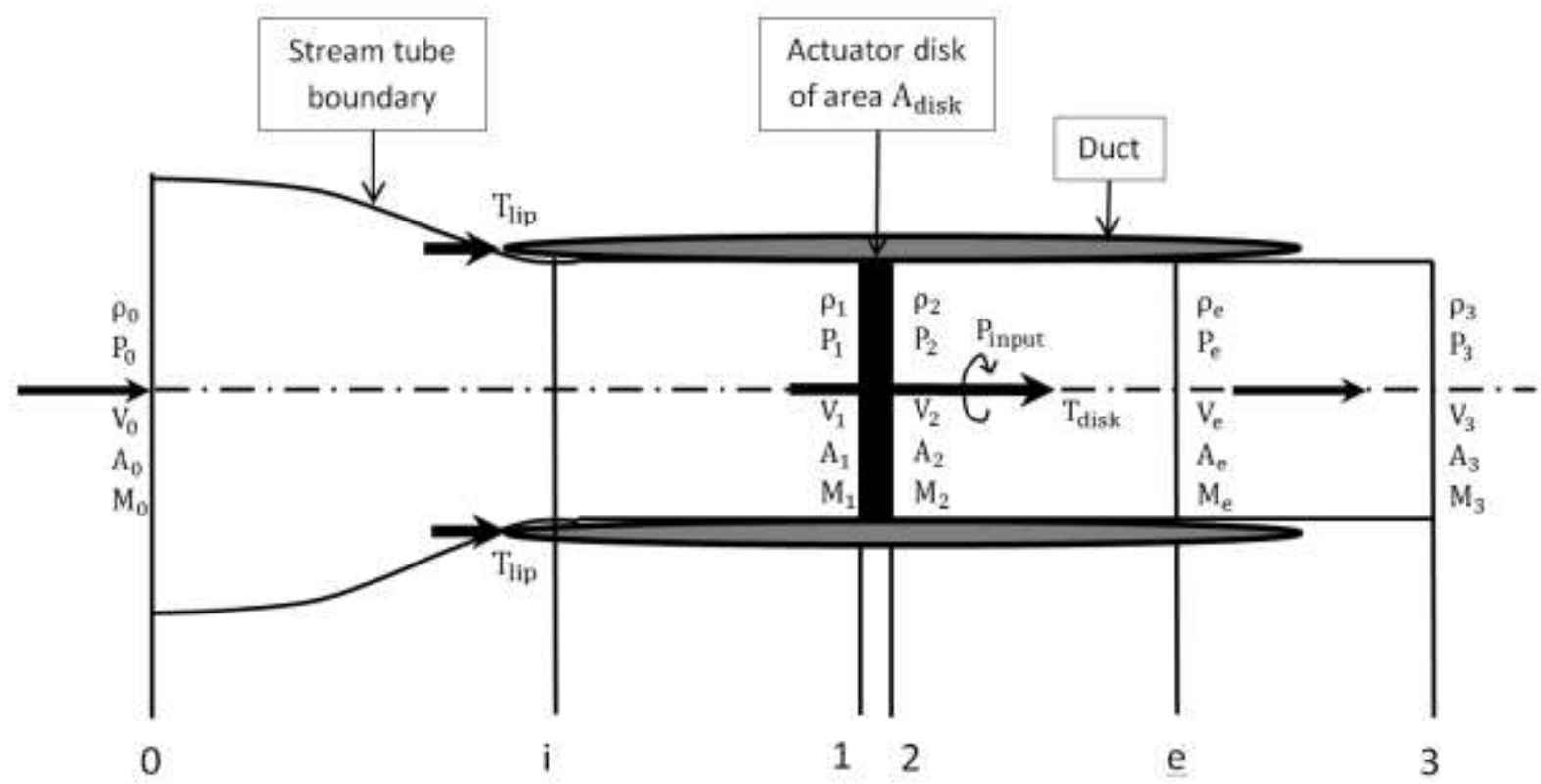

Figure 15. Schematic of the ducted, compressible Actuator Disk Theory.

This section will focus on establishing the governing equations specific to the ducted case of the compressible Actuator Disk Theory, following the assumptions made in section 2.1. The actuator disk is again assumed to be an energy-adding device that adds power to the flow and exerts a thrust force on the flow in the direction of the flow. The actuator disk is now also enclosed by a duct of the same cross-sectional area as the disk as shown in Figure 15. Stations 0 and 3 represents the far upstream and downstream; stations i and e represents the duct inlet and outlet; and stations 1 and 2 are immediately before and after the disk. As mentioned in Chapter 1, the duct can be of variable cross-sectional area, but it is assumed to have constant area to keep the scope of the thesis manageable. The constant area duct entails that the inlet and outlet duct areas as well as the area at the far downstream area equal to the disk area

$$
A_{i}=A_{1}=A_{2}=A_{\text {disk }}=A_{e}=A_{3}
$$

In addition, the duct is also assumed to produce straight and parallel streamlines at the duct exit such that the exit pressure is considered equal to the far downstream pressure, which in turn is equal to the free-stream pressure. 


$$
\mathrm{P}_{\mathrm{e}}=\mathrm{P}_{3}=\mathrm{P}_{0}
$$

With $\mathrm{P}_{\mathrm{e}}=\mathrm{P}_{3}$, it is also apparent that $\rho_{\mathrm{e}}$ is equal to $\rho_{3}$ from the isentropic pressure-density relation

$$
\begin{gathered}
\frac{\rho_{\mathrm{e}}}{\rho_{3}}=\left(\frac{\mathrm{P}_{\mathrm{e}}}{\mathrm{P}_{3}}\right)^{\frac{1}{\gamma}}=1 \\
\therefore \rho_{\mathrm{e}}=\rho_{3}
\end{gathered}
$$

With $\rho_{\mathrm{e}}=\rho_{3}$ and $\mathrm{A}_{\mathrm{e}}=\mathrm{A}_{3}$, the conservation of mass applied to control volume e- 3 indicates that $V_{\mathrm{e}}$ is also equal to $V_{3}$

$$
\begin{gathered}
\rho_{\mathrm{e}} \mathrm{A}_{\mathrm{e}} \mathrm{V}_{\mathrm{e}}=\rho_{3} \mathrm{~A}_{3} \mathrm{~V}_{3} \\
\therefore \mathrm{V}_{\mathrm{e}}=\mathrm{V}_{3}
\end{gathered}
$$

Lastly, it can be shown that $\mathrm{M}_{\mathrm{e}}=\mathrm{M}_{3}$ using the isentropic pressure ratio

$$
\begin{gathered}
\frac{\mathrm{P}_{3}}{\mathrm{P}_{\mathrm{e}}}=\left(\frac{1+\frac{\gamma-1}{2} \mathrm{M}_{\mathrm{e}}^{2}}{1+\frac{\gamma-1}{2} \mathrm{M}_{3}^{2}}\right)^{\frac{\gamma}{\gamma-1}}=1 \\
\therefore \mathrm{M}_{\mathrm{e}}=\mathrm{M}_{3}
\end{gathered}
$$

Therefore, since all the flow variables and the area at the duct exit and the far downstream are equal, station e can be replaced entirely by station 3, and this will be done for the rest of the analysis. As a result, the governing equations from 2-29 to 2-43 that were derived for the compressible bare Actuator Disk Theory also apply to the current analysis of the ducted case without any change of subscripts. Furthermore, the assumption of constant duct area simplifies the isentropic flow relations between stations 2 and 3. Since $A_{\text {disk }}=A_{3}$, the isentropic relation between area and Mach number shows that $\mathrm{M}_{2}$ is equal to 3 .

$$
\begin{gathered}
\frac{\mathrm{A}_{3}}{\mathrm{~A}_{\text {disk }}}=1=\frac{\mathrm{M}_{2}}{\mathrm{M}_{3}}\left(\frac{1+\frac{\gamma-1}{2} \mathrm{M}_{3}^{2}}{1+\frac{\gamma-1}{2} \mathrm{M}_{2}^{2}}\right)^{\frac{\gamma+1}{2(\gamma-1)}} \\
\therefore \mathrm{M}_{2}=\mathrm{M}_{3}
\end{gathered}
$$

With $M_{2}$ equal to $M_{3}$, the isentropic relation between pressure and Mach number shows that $P_{2}$ is equal to $\mathrm{P}_{3}$. 


$$
\begin{gathered}
\frac{\mathrm{P}_{3}}{\mathrm{P}_{2}}=\left(\frac{1+\frac{\gamma-1}{2} \mathrm{M}_{2}^{2}}{1+\frac{\gamma-1}{2} \mathrm{M}_{3}^{2}}\right)^{\frac{\gamma}{\gamma-1}}=1 \\
\therefore \mathrm{P}_{2}=\mathrm{P}_{3}
\end{gathered}
$$

The implication that $P_{2}$ is equal to $P_{3}$ further suggests that $\rho_{2}$ is equal to $\rho_{3}$ when considering the isentropic pressure-density relation

$$
\begin{gathered}
\frac{\rho_{2}}{\rho_{3}}=\left(\frac{P_{2}}{P_{3}}\right)^{\frac{1}{\gamma}}=1 \\
\therefore \rho_{2}=\rho_{3}
\end{gathered}
$$

Since $\rho_{2}$ is equal to $\rho_{3}$, the conservation of mass conveys that $V_{2}$ is also equal to $V_{3}$.

$$
\begin{gathered}
\rho_{2} \mathrm{~A}_{2} \mathrm{~V}_{2}=\rho_{3} \mathrm{~A}_{3} \mathrm{~V}_{3} \\
\therefore \mathrm{V}_{2}=\mathrm{V}_{3}
\end{gathered}
$$

Since all the flow variables and the area immediately after the disk and the far downstream are equal, it can be stated that the flow properties after the disk remain constant all throughout the remaining section of the duct and out to the far downstream. 


\subsubsection{Lip Thrust Analysis}

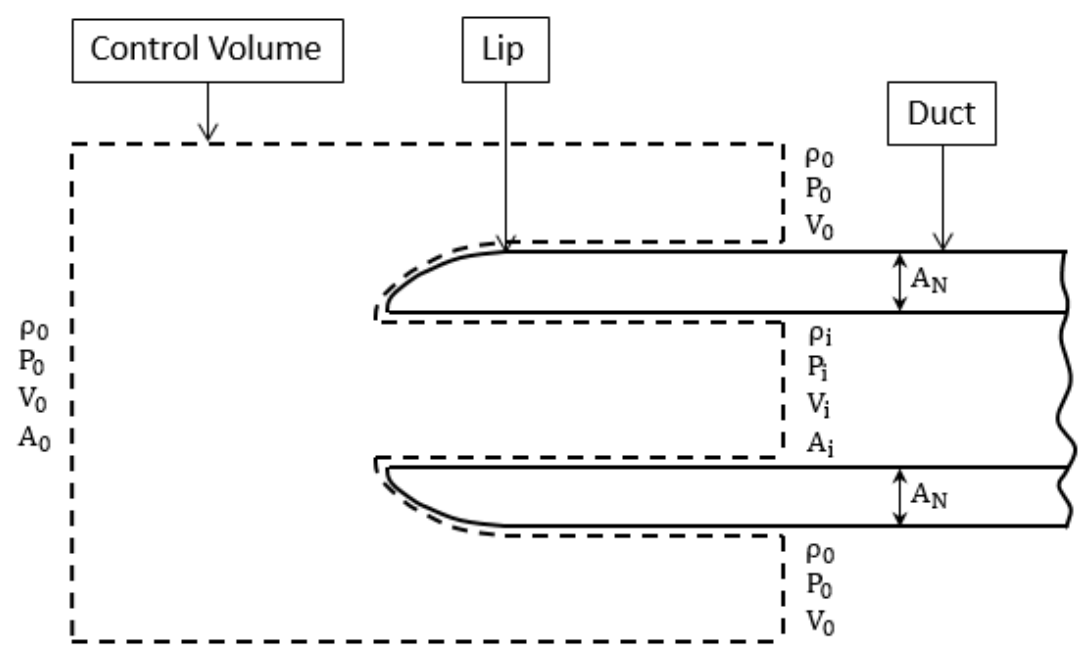

Figure 16. Control volume used to drive lip thrust.

The addition of the constant area duct onto the disk assembly also introduces the concept of lip thrust much like in the case of the ducted incompressible Actuator Disk Theory. As mentioned in Chapter 1, Küchemann and Weber [3] have derived this lip thrust by applying the conservation of momentum to the control volume enclosing the far upstream and the duct inlet as shown in Figure 16, where the subscript 0 denotes the far upstream and the subscript $\mathrm{i}$ denotes the inlet at some distance behind the lip. The resulting momentum equation is given below

$$
\int_{A_{N}}\left(P_{N}-P_{0}\right) d A_{N}=\rho_{i} V_{i} A_{i}\left(V_{0}-V_{i}\right)-\left(P_{i}-P_{0}\right) A_{i}
$$

where the lip thrust is the negative of the integral and is the force that the duct inlet exerts on the flow.

$$
\begin{gathered}
\mathrm{T}_{\text {lip }}=-\int_{\mathrm{A}_{\mathrm{N}}}\left(\mathrm{P}_{\mathrm{N}}-\mathrm{P}_{0}\right) \mathrm{d} \mathrm{A}_{\mathrm{N}} \\
\mathrm{T}_{\text {lip }}=-\rho_{\mathrm{i}} \mathrm{V}_{\mathrm{i}} \mathrm{A}_{\mathrm{i}}\left(\mathrm{V}_{0}-\mathrm{V}_{\mathrm{i}}\right)+\left(\mathrm{P}_{\mathrm{i}}-\mathrm{P}_{0}\right) \mathrm{A}_{\mathrm{i}}
\end{gathered}
$$

Note that the above lip thrust equation, resulting from the momentum balance, is general and can be applied to both compressible and incompressible flow. It will be tailored to the compressible ducted case in the next steps.

Factoring $\mathrm{V}_{0}$ from the first term and $\mathrm{P}_{0}$ from the second term gives 


$$
\mathrm{T}_{\text {lip }}=-\rho_{\mathrm{i}} \mathrm{V}_{\mathrm{i}} \mathrm{A}_{\mathrm{i}} \mathrm{V}_{0}\left(1-\frac{\mathrm{V}_{\mathrm{i}}}{\mathrm{V}_{0}}\right)+\mathrm{P}_{0} \mathrm{~A}_{\mathrm{i}}\left(\frac{\mathrm{P}_{\mathrm{i}}}{\mathrm{P}_{0}}-1\right)
$$

To non-dimensionalize the lip thrust, both sides of the equation are divided by the reference force

$$
\frac{\mathrm{T}_{\text {lip }}}{\frac{1}{2} \rho_{0} \mathrm{~V}_{0}^{2} \mathrm{~A}_{\mathrm{i}}}=-\frac{\rho_{\mathrm{i}} \mathrm{V}_{\mathrm{i}} \mathrm{A}_{\mathrm{i}} \mathrm{V}_{0}}{\frac{1}{2} \rho_{0} \mathrm{~V}_{0}^{2} \mathrm{~A}_{\mathrm{i}}}\left(1-\frac{\mathrm{V}_{\mathrm{i}}}{\mathrm{V}_{0}}\right)+\frac{\mathrm{P}_{0} \mathrm{~A}_{\mathrm{i}}}{\frac{1}{2} \rho_{0} \mathrm{~V}_{0}^{2} \mathrm{~A}_{\mathrm{i}}}\left(\frac{\mathrm{P}_{\mathrm{i}}}{\mathrm{P}_{0}}-1\right)
$$

and simplified to give

$$
\frac{\mathrm{T}_{\text {lip }}}{\frac{1}{2} \rho_{0} \mathrm{~V}_{0}^{2} \mathrm{~A}_{\mathrm{i}}}=2 \frac{\rho_{\mathrm{i}}}{\rho_{0}} \frac{\mathrm{V}_{\mathrm{i}}}{\mathrm{V}_{0}}\left(\frac{\mathrm{V}_{\mathrm{i}}}{\mathrm{V}_{0}}-1\right)+2 \frac{\mathrm{P}_{0}}{\rho_{0} \mathrm{~V}_{0}^{2}}\left(\frac{\mathrm{P}_{\mathrm{i}}}{\mathrm{P}_{0}}-1\right)
$$

Using the isentropic speed of sound relation,

$$
\mathrm{a}_{0}^{2}=\frac{\gamma \mathrm{P}_{0}}{\rho_{0}}
$$

along with the definition of the Mach number,

$$
\mathrm{M}_{0}=\frac{\mathrm{V}_{0}}{\mathrm{a}_{0}}
$$

the lip thrust equation can be expressed as follows

$$
\frac{\mathrm{T}_{\text {lip }}}{\frac{1}{2} \rho_{0} \mathrm{~V}_{0}^{2} \mathrm{~A}_{\mathrm{i}}}=2 \frac{\rho_{\mathrm{i}}}{\rho_{0}} \frac{\mathrm{V}_{\mathrm{i}}}{\mathrm{V}_{0}}\left(\frac{\mathrm{V}_{\mathrm{i}}}{\mathrm{V}_{0}}-1\right)+\frac{2}{\gamma \mathrm{M}_{0}^{2}}\left(\frac{\mathrm{P}_{\mathrm{i}}}{\mathrm{P}_{0}}-1\right)
$$

The last step of the manipulation is to put the non-dimensional lip thrust in terms of free-stream and inlet velocities. This is done by using the compressible energy equation, $\mathrm{E}_{0}=\mathrm{E}_{\mathrm{i}}$ :

$$
\frac{\gamma}{\gamma-1} \frac{\mathrm{P}_{0}}{\rho_{0}}+\frac{\mathrm{V}_{0}^{2}}{2}=\frac{\gamma}{\gamma-1} \frac{\mathrm{P}_{\mathrm{i}}}{\rho_{\mathrm{i}}}+\frac{\mathrm{V}_{\mathrm{i}}^{2}}{2}
$$

along with the isentropic pressure-density ratio

$$
\frac{\mathrm{P}_{\mathrm{i}}}{\mathrm{P}_{0}}=\left(\frac{\rho_{\mathrm{i}}}{\rho_{0}}\right)^{\gamma}
$$

to develop relations for the pressure and density ratios as shown.

$$
\begin{aligned}
& \frac{\mathrm{P}_{\mathrm{i}}}{\mathrm{P}_{0}}=\left(1+\frac{\gamma-1}{2} \mathrm{M}_{0}^{2}\left(1-\left(\frac{\mathrm{V}_{\mathrm{i}}}{\mathrm{V}_{0}}\right)^{2}\right)\right)^{\frac{\gamma}{\gamma-1}} \\
& \frac{\rho_{\mathrm{i}}}{\rho_{0}}=\left(1+\frac{\gamma-1}{2} \mathrm{M}_{0}^{2}\left(1-\left(\frac{\mathrm{V}_{\mathrm{i}}}{\mathrm{V}_{0}}\right)^{2}\right)\right)^{\frac{1}{\gamma-1}}
\end{aligned}
$$


Lastly, the pressure and density ratios are substituted back into the equation to give the final nondimensional lip thrust in terms of $M_{0}, \gamma$, and the velocity ratio $\frac{V_{i}}{V_{0}}$

$$
\begin{aligned}
\frac{\mathrm{T}_{\text {lip }}}{\frac{1}{2} \rho_{0} \mathrm{~V}_{0}^{2} \mathrm{~A}_{\mathrm{i}}} & =2\left(\frac{\mathrm{V}_{\mathrm{i}}}{\mathrm{V}_{0}}\right)\left(\frac{\mathrm{V}_{\mathrm{i}}}{\mathrm{V}_{0}}-1\right)\left(1+\frac{\gamma-1}{2} \mathrm{M}_{0}^{2}\left(1-\left(\frac{\mathrm{V}_{\mathrm{i}}}{\mathrm{V}_{0}}\right)^{2}\right)\right)^{\frac{1}{\gamma-1}} \\
& +\frac{2}{\gamma \mathrm{M}_{0}^{2}}\left(\left(1+\frac{\gamma-1}{2} \mathrm{M}_{0}^{2}\left(1-\left(\frac{\mathrm{V}_{\mathrm{i}}}{\mathrm{V}_{0}}\right)^{2}\right)\right)^{\frac{\gamma}{\gamma-1}}-1\right)
\end{aligned}
$$

Note that when the ratio of the duct inlet to the far upstream velocity $\frac{V_{i}}{V_{0}}$ is equal to 1 , the lip thrust goes to zero, which is consistent with the lip thrust for incompressible flow.

Assuming the streamlines are straight and parallel so that the pressure at station 1 is the same as that at the inlet, the flow variables at stations $i$ and 1 are equal for the same reasons that the flow variables at stations e and 3 are equal. As a result, replacing the subscript i in equation 2-73 with 1 and asserting that $A_{1}=A_{\text {disk }}$ gives

$$
\begin{aligned}
\frac{\mathrm{T}_{\text {lip }}}{\frac{1}{2} \rho_{0} \mathrm{~V}_{0}^{2} \mathrm{~A}_{\text {disk }}} & =2\left(\frac{\mathrm{V}_{1}}{\mathrm{~V}_{0}}\right)\left(\frac{\mathrm{V}_{1}}{\mathrm{~V}_{0}}-1\right)\left(1+\frac{\gamma-1}{2} \mathrm{M}_{0}^{2}\left(1-\left(\frac{\mathrm{V}_{1}}{\mathrm{~V}_{0}}\right)^{2}\right)\right)^{\frac{1}{\gamma-1}} \\
+\frac{2}{\gamma \mathrm{M}_{0}^{2}} & \left(\left(1+\frac{\gamma-1}{2} \mathrm{M}_{0}^{2}\left(1-\left(\frac{\mathrm{V}_{1}}{\mathrm{~V}_{0}}\right)^{2}\right)\right)^{\frac{\gamma}{\gamma-1}}-1\right)
\end{aligned}
$$

Alternately, the lip thrust can also be derived by applying the static equilibrium equation in the direction of the flow on the disk-and-duct assembly. By doing so, the lip thrust is also the difference between the total and the disk thrust.

$$
\mathrm{T}_{\text {lip }}=\mathrm{T}_{\text {total }}-\mathrm{T}_{\text {disk }}
$$

Logically, the lip thrust from this derivation must equal the lip thrust determined by Küchemann and Weber [3] using the control volume encasing the flow from the far upstream to the duct inlet. To have a more complete and collective analysis of the ducted case of compressible Actuator Disk Theory, it is shown in Appendix B that it is indeed the case. This concludes the derivation 
of the governing equations for the ducted case of the compressible Actuator Disk Theory, which are summarized in the following section.

\subsubsection{Summary of Governing Equations}

In summary, the assumption of constant duct area implies the following for the ducted case of the compressible Actuator Disk Theory.

$$
\begin{gathered}
\mathrm{M}_{2}=\mathrm{M}_{\mathrm{e}}=\mathrm{M}_{3} \\
\mathrm{~V}_{2}=\mathrm{V}_{\mathrm{e}}=\mathrm{V}_{3} \\
\mathrm{P}_{0}=\mathrm{P}_{2}=\mathrm{P}_{\mathrm{e}}=\mathrm{P}_{3} \\
\rho_{0}=\rho_{2}=\rho_{\mathrm{e}}=\rho_{3}
\end{gathered}
$$

Due to these results from the assumption of constant duct area, the governing equations are reduced to the following below. These governing equations relate the flow variables at each station, the mass flowrate, the power addition by the disk, and the total, disk, and lip thrusts to the free-stream flow conditions which are $\gamma, \mathrm{P}_{0}, \rho_{0}$, and $\mathrm{V}_{0}$ and the disk area.

$$
\begin{gathered}
\mathrm{M}_{0}=\mathrm{V}_{0}\left(\gamma \cdot \frac{\mathrm{P}_{0}}{\rho_{0}}\right)^{-\frac{1}{2}} \\
\frac{\mathrm{A}_{0}}{\mathrm{~A}_{\mathrm{disk}}}=\frac{\mathrm{M}_{1}}{\mathrm{M}_{0}}\left(\frac{1+\frac{\gamma-1}{2} \mathrm{M}_{0}^{2}}{1+\frac{\gamma-1}{2} \mathrm{M}_{1}^{2}}\right)^{\frac{\gamma+1}{2(\gamma-1)}} \\
\frac{\mathrm{P}_{1}}{\mathrm{P}_{0}}=\left(\frac{1+\frac{\gamma-1}{2} \mathrm{M}_{0}^{2}}{1+\frac{\gamma-1}{2} \mathrm{M}_{1}^{2}}\right)^{\frac{\gamma}{\gamma-1}} \\
\frac{\rho_{1}}{\rho_{0}}=\left(\frac{\mathrm{P}_{1}}{\mathrm{P}_{0}}\right)^{\frac{1}{\gamma}} \\
\frac{\mathrm{V}_{1}}{\mathrm{~V}_{0}}=\frac{\rho_{0}}{\rho_{1}} \frac{\mathrm{A}_{0}}{\mathrm{~A}_{\mathrm{disk}}} \\
\mathrm{M}_{3}=\mathrm{V}_{3}\left(\gamma \cdot \frac{\mathrm{P}_{0}}{\rho_{0}}\right)^{-\frac{1}{2}} \\
\dot{\mathrm{m}}=\rho_{1} \mathrm{~A}_{\text {disk }} \mathrm{V}_{1}
\end{gathered}
$$




$$
\begin{gathered}
\frac{\mathrm{T}_{\text {lip }}}{\frac{1}{2} \rho_{0} \mathrm{~V}_{0}^{2} \mathrm{~A}_{\text {disk }}}=2\left(\frac{\mathrm{V}_{1}}{\mathrm{~V}_{0}}\right)\left(\frac{\mathrm{V}_{1}}{\mathrm{~V}_{0}}-1\right)\left(1+\frac{\gamma-1}{2} \mathrm{M}_{0}^{2}\left(1-\left(\frac{\mathrm{V}_{1}}{\mathrm{~V}_{0}}\right)^{2}\right)\right)^{\frac{1}{\gamma-1}} \\
+\frac{2}{\gamma \mathrm{M}_{0}^{2}}\left(\left(1+\frac{\gamma-1}{2} \mathrm{M}_{0}^{2}\left(1-\left(\frac{\mathrm{V}_{1}}{\mathrm{~V}_{0}}\right)^{2}\right)\right)^{\frac{\gamma}{\gamma-1}}-1\right) \\
\mathrm{T}_{\text {total }}=\dot{\mathrm{m}}\left(\mathrm{V}_{3}-\mathrm{V}_{0}\right) \\
\mathrm{T}_{\text {disk }}=\dot{\mathrm{m}}\left(\mathrm{V}_{2}-\mathrm{V}_{1}\right)+\mathrm{A}_{\text {disk }}\left(\mathrm{P}_{2}-\mathrm{P}_{1}\right) \\
\mathrm{T}_{\text {lip }}=\mathrm{T}_{\text {total }}-\mathrm{T}_{\text {disk }} \\
\mathrm{P}_{\text {input }}=\dot{\mathrm{m}} \frac{\mathrm{V}_{3}^{2}-\mathrm{V}_{0}^{2}}{2}
\end{gathered}
$$

In comparison, the assumption of the constant duct area gives the same results for both the incompressible and compressible Actuator Disk Theory where the flow properties are constant from the immediate downstream of the disk, throughout the remaining section of the duct and out to the far downstream. The disk thrust contains an additional term due to the discontinuity in velocity across the disk, while the total thrust and the power addition given in equation 2-88 and 2-91 are identical to those for the ducted case of the classical Actuator Disk Theory. Lastly, the lip thrust cannot be expressed in terms of the far downstream velocity as can be done for incompressible flow due to the fact that velocity is discontinuous across the disk. Next, the method for determining the flow variables and the disk performance will be discussed. 


\subsection{Method of Solution}

For both the compressible and classical Actuator Disk Theory, the governing equations provide a system of equations necessary to determine the flow variables at each station and the performance of the actuator disk in terms of some given inputs. The inputs are the free-stream flow conditions which are $\gamma, P_{0}, \rho_{0}$, and $V_{0}$ and the disk cross-sectional area $A_{\text {disk. }}$. Along with these inputs, an additional variable is specified depending on whether energy is added to or extracted from the flow. Usually, this independent variable is the power addition to the flow in the case of an energy-adding device and the far downstream velocity in the case of an energy-extracting device.

As discussed in Chapter 1, the unknown variables in the governing equations for the classical Actuator Disk Theory can be expressed solely in terms of the given inputs and be solved explicitly, which is not true for the compressible theory. Some of the flow variables in the governing equations for the compressible theory cannot be isolated such as $M_{1}$ in the isentropic area-to-Mach relation between station 0 and 1 in equation 2-30. As a result, the governing equations are a mixture of both inputs and outputs on the right side of the equations; whereas, the incompressible-flow equations have the outputs separated to the left side and the inputs to right side.

Therefore, the compressible-flow equations, for both the bare and ducted case, cannot be solved explicitly and require an iterative method. One iterative method has been presented and employed by Delano and Crigler [5] to obtain the compressible-flow solutions for the bare propeller, which is an energy-adding device. In addition to specifying the power addition by the disk $\mathrm{P}_{\text {input }}$, the free-stream flow conditions $\gamma, \mathrm{P}_{0}, \rho_{0}$, and $\mathrm{V}_{0}$, and the disk area $\mathrm{A}_{\text {disk }}$, the Mach number immediately before the disk $\mathrm{M}_{1}$ is also initially specified to begin marching through the compressible equations listed in section 2.2.1 (equations 2-29 to 2-44) to determine the flow variables at each station. The flow variables associated with the assumed $\mathrm{M}_{1}$ are then used to solve for the total thrust $\mathrm{T}_{\text {total }}$ and the thrust at the disk plane $\mathrm{T}_{\text {disk }}$ separately, which are also 
equal to each other for the bare actuator disk. If $\mathrm{T}_{\text {total }}$ and $\mathrm{T}_{\text {disk }}$ are not equal, then the process of assuming a new $M_{1}$ and solving for the flow variables with the assumed $M_{1}$ is repeated until $\mathrm{T}_{\text {total }}$ and $\mathrm{T}_{\mathrm{disk}}$ are approximately equal. The same iterative approach can also be taken for the ducted case of compressible Actuator Disk Theory, where the total thrust $\mathrm{T}_{\text {total }}$, the disk thrust $\mathrm{T}_{\text {disk }}$, and the lip thrust $\mathrm{T}_{\text {lip }}$ can be found separately for the assumed $\mathrm{M}_{1}$. Then, a new $\mathrm{M}_{1}$ is assumed until the lip thrust is equal to the difference between total and disk thrust: $\mathrm{T}_{\text {lip }}=\mathrm{T}_{\text {total }}-$ $\mathrm{T}_{\text {disk }}$.

Due to the accessibility of numerical computing software today, the iterative method is implemented into EES (Engineering Equation Solver), a program that can solve numerous coupled non-linear algebraic and differential equations using numerical methods. Thus, by providing the same number of equations as the number of unknowns into EES, all compressibleflow solutions presented in this thesis work can be determined. 


\section{APPLICATIONS OF THE COMPRESSIBLE ACTUATOR DISK THEORY - \\ PROPELLER}

\subsection{Introduction}

In this chapter, the governing equations derived for the bare and ducted case of the compressible Actuator Disk Theory in Chapter 2 will be applied to a propeller. A propeller is considered an energy-adding device, adding power to the flow stream at the disk plane and exerting a thrust force on the flow in the direction of the flow. Its purpose is to generate high propulsive thrust for a given power input; hence, a well-accepted measure of its performance is its propulsive efficiency against the power addition to the flow by the propeller. The dimensionless quantities such as the propulsive efficiency and the thrust and power coefficients are developed in this chapter for the bare and ducted propeller.

The governing equations for the compressible Actuator Disk Theory are used to determine the flow variables at each station and the performance of both the bare and ducted propeller in terms of the free-stream flow conditions, the disk area, and the power coefficient. The solutions to the compressible bare and ducted propeller are made dimensionless by the free-stream conditions and are compared with the results obtained by Delano and Crigler [5] for agreement.

To begin, the propeller is assumed to have an infinite number of blades such that it becomes an actuator disk with an area of $\mathrm{A}_{\text {disk }}$. Because the governing equations for the compressible Actuator Disk Theory are derived in Chapter 2 already assuming that the disk is an energy-adding device, much like a propeller, they can be utilized here without any sign change, starting with the case of the bare propeller. 


\subsection{Compressible, Bare Propeller}

The dimensionless parameters - the power coefficient, the total thrust coefficient, and the propulsive efficiency — will be established for the compressible, bare propeller. The power coefficient is found by dividing the power addition to the flow in equation 2-43 by the available power in the free-stream in an area of $\mathrm{A}_{\text {disk }}$,

$$
C_{P}=\frac{P_{\text {input }}}{P_{\text {available }}}=\frac{\frac{1}{2} \dot{m}\left(V_{3}^{2}-V_{0}^{2}\right)}{\frac{1}{2} \rho_{0} A_{\text {disk }} V_{0}^{3}}
$$

Similarly, the total thrust coefficient is found by dividing the total thrust in equation $2-41$ by the reference force

$$
\mathrm{C}_{\mathrm{T}}=\frac{\mathrm{T}_{\text {total }}}{\frac{1}{2} \rho_{0} A_{\text {disk }} V_{0}^{2}}=\frac{\dot{m}\left(V_{3}-V_{0}\right)}{\frac{1}{2} \rho_{0} A_{\text {disk }} V_{0}^{2}}
$$

Lastly, the propulsive efficiency $\eta_{\text {prop }}$ is the ratio of the propulsive power to the total power added by the propeller where the propulsive power is the product of the total thrust and the freestream velocity

$$
\eta_{\text {prop }}=\frac{\text { propulsive power }}{P_{\text {input }}}=\frac{\dot{\mathrm{m}}\left(\mathrm{V}_{3}-\mathrm{V}_{0}\right) \mathrm{V}_{0}}{\frac{1}{2} \mathrm{~m}\left(\mathrm{~V}_{3}^{2}-\mathrm{V}_{0}^{2}\right)}
$$

By simplifying, the propulsive efficiency is given in terms of the velocity ratio $\frac{V_{3}}{V_{0}}$

$$
\eta_{\text {prop }}=\frac{2 \mathrm{~V}_{0}}{\mathrm{~V}_{3}+\mathrm{V}_{0}}=\frac{2}{\frac{\mathrm{V}_{3}}{\mathrm{~V}_{0}}+1}
$$

Alternatively, the propulsive efficiency is also the ratio of the total thrust coefficient to the power coefficient

$$
\eta_{\text {prop }}=\frac{C_{T}}{C_{p}}=\frac{2 \frac{A_{0}}{A_{\text {disk }}}\left(\frac{V_{3}}{V_{0}}-1\right)}{\frac{A_{0}}{A_{\text {disk }}}\left(\left(\frac{V_{3}}{V_{0}}\right)^{2}-1\right)}
$$

By canceling the area ratio $\frac{A_{0}}{A_{\text {disk }}}$ in the numerator and the denominator of equation 3-5 and expanding the denominator, the propulsive efficiency is

$$
\eta_{\text {prop }}=\frac{2\left(\frac{\mathrm{v}_{3}}{\mathrm{v}_{0}}-1\right)}{\left(\frac{\mathrm{v}_{3}}{\mathrm{v}_{0}}-1\right)\left(\frac{\mathrm{V}_{3}}{\mathrm{v}_{0}}+1\right)}
$$


Simplifying equation 3-6will give the propulsive efficiency in equation 3-4. An alternative form of the power coefficient and the total thrust coefficient will be shown.

By substituting the following mass flowrate

$$
\dot{\mathrm{m}}=\rho_{0} \mathrm{~A}_{0} \mathrm{~V}_{0}
$$

into equation 3-1 and simplifying, the power coefficient becomes

$$
C_{p}=\frac{A_{0}}{A_{\text {disk }}}\left(\left(\frac{V_{3}}{V_{0}}\right)^{2}-1\right)
$$

Equation 3-8 can be rearranged to give the following velocity ratio as a function of the power coefficient $C_{P}$ and the ratio between the free-stream and the disk area $\frac{A_{0}}{A_{\text {disk }}}$

$$
\frac{\mathrm{V}_{3}}{\mathrm{~V}_{0}}=\left(1+\frac{\mathrm{C}_{\mathrm{P}}}{\frac{\mathrm{A}_{0}}{\mathrm{~A}_{\mathrm{disk}}}}\right)^{\frac{1}{2}}
$$

Likewise, the thrust coefficient can be simplified to the following form by using the mass flowrate given in equation 3-7

$$
\mathrm{C}_{\mathrm{T}}=2 \frac{\mathrm{A}_{0}}{\mathrm{~A}_{\mathrm{disk}}}\left(\frac{\mathrm{V}_{3}}{\mathrm{~V}_{0}}-1\right)
$$

Although the form of the power coefficient and the total thrust coefficient given in equations 3-8 and 3-10 is unconventional, it is useful when comparing the results that are presented later in the chapter.

\subsubsection{Summary of Governing Equations}

The complete set of the governing equations, including the power coefficient, the total thrust coefficient, and the propulsive efficiency, for the compressible bare propeller are given. Note that these equations are a non-linear, coupled algebraic set.

$$
\begin{gathered}
M_{0}=V_{0}\left(\gamma \cdot \frac{P_{0}}{\rho_{0}}\right)^{-\frac{1}{2}} \\
\frac{A_{0}}{A_{\text {disk }}}=\frac{M_{1}}{M_{0}}\left(\frac{1+\frac{\gamma-1}{2} M_{0}^{2}}{1+\frac{\gamma-1}{2} M_{1}^{2}}\right)^{\frac{\gamma+1}{2(\gamma-1)}}
\end{gathered}
$$




$$
\begin{aligned}
& \frac{\mathrm{P}_{1}}{\mathrm{P}_{0}}=\left(\frac{1+\frac{\gamma-1}{2} \mathrm{M}_{0}^{2}}{1+\frac{\gamma-1}{2} \mathrm{M}_{1}^{2}}\right)^{\frac{\gamma}{\gamma-1}} \\
& \frac{\rho_{1}}{\rho_{0}}=\left(\frac{\mathrm{P}_{1}}{\mathrm{P}_{0}}\right)^{\frac{1}{\gamma}} \\
& \frac{\mathrm{V}_{1}}{\mathrm{~V}_{0}}=\frac{\rho_{0}}{\rho_{1}} \frac{\mathrm{A}_{0}}{\mathrm{~A}_{\mathrm{disk}}} \\
& M_{3}=V_{3}\left(\gamma \cdot \frac{P_{0}}{\rho_{0}}\right)^{-\frac{1}{2}} \\
& \frac{A_{3}}{A_{0}}=\frac{V_{0}}{V_{3}} \\
& \frac{A_{3}}{A_{\text {disk }}}=\frac{M_{2}}{M_{3}}\left(\frac{1+\frac{\gamma-1}{2} M_{3}^{2}}{1+\frac{\gamma-1}{2} M_{2}^{2}}\right)^{\frac{\gamma+1}{2(\gamma-1)}} \\
& \frac{\mathrm{P}_{2}}{\mathrm{P}_{0}}=\left(\frac{1+\frac{\gamma-1}{2} \mathrm{M}_{3}^{2}}{1+\frac{\gamma-1}{2} \mathrm{M}_{2}^{2}}\right)^{\frac{\gamma}{\gamma-1}} \\
& \frac{\rho_{2}}{\rho_{0}}=\left(\frac{\mathrm{P}_{2}}{\mathrm{P}_{0}}\right)^{\frac{1}{\gamma}} \\
& \mathrm{V}_{2}=\frac{\rho_{1}}{\rho_{2}} \mathrm{~V}_{1} \\
& \dot{\mathrm{m}}=\rho_{1} \mathrm{~A}_{\mathrm{disk}} \mathrm{V}_{1} \\
& \mathrm{~T}_{\text {total }}=\dot{\mathrm{m}}\left(\mathrm{V}_{3}-\mathrm{V}_{0}\right) \\
& \mathrm{T}_{\text {prop }}=\dot{\mathrm{m}}\left(\mathrm{V}_{2}-\mathrm{V}_{1}\right)+\mathrm{A}_{\text {disk }}\left(\mathrm{P}_{2}-\mathrm{P}_{1}\right) \\
& \mathrm{T}_{\text {total }}=\mathrm{T}_{\text {prop }} \\
& \frac{\mathrm{V}_{3}}{\mathrm{~V}_{0}}=\left(1+\frac{\mathrm{C}_{\mathrm{P}}}{\frac{\mathrm{A}_{0}}{\mathrm{~A}_{\mathrm{disk}}}}\right)^{\frac{1}{2}} \\
& \mathrm{P}_{\text {input }}=\dot{\mathrm{m}} \frac{\mathrm{V}_{3}^{2}-\mathrm{V}_{0}^{2}}{2} \\
& \mathrm{C}_{\mathrm{T}}=2 \frac{\mathrm{A}_{0}}{\mathrm{~A}_{\mathrm{disk}}}\left(\frac{\mathrm{V}_{3}}{\mathrm{~V}_{0}}-1\right) \\
& \eta_{\text {prop }}=\frac{2}{\frac{v_{3}}{v_{0}}+1}
\end{aligned}
$$


Given the free-stream conditions, the disk area, and the power addition to the flow, these governing equations provide a system of equations to determine the flow variables before the disk and throughout the slipstream and the performance of the bare propeller.

\subsubsection{Method of Solution}

The purpose of the current analysis is to solve for the flow variables at stations 0 to 4 and to determine the performance of the bare propeller using the governing equations given in section 3.2.1. To do so, the disk area $A_{\text {disk }}$, the power addition to the flow $P_{\text {input }}$, and the free-stream conditions $\gamma, \rho_{0}, P_{0}$, and $V_{0}$ are specified. With these six inputs, $\gamma, \rho_{0}, P_{0}, V_{0}, A_{\text {disk }}$, and $P_{\text {input }}$, the unknown variables left to solve are the following flow variables $A_{0}, M_{0}, M_{1}, \rho_{1}, P_{1}, V_{1}, M_{2}, \rho_{2}, P_{2}$, $\mathrm{V}_{2}, \mathrm{~A}_{3}, \mathrm{M}_{3}$, and $\mathrm{V}_{3}$ and the performance parameters of the bare propeller $\mathrm{T}_{\text {prop }}, \mathrm{T}_{\text {total }}, \mathrm{C}_{\mathrm{P}}, \mathrm{C}_{\mathrm{T}}$, $\eta_{\text {prop}}$, and $\dot{\mathrm{m}}$, giving a total of nineteen unknown variables. The governing equations in section 3.2.1 give the necessary nineteen equations to solve for these nineteen unknown variables.

Next, the dimensionless independent variables are determined using Buckingham Pi Theorem to express the propulsive efficiency of the bare propeller as a function of the given variables $\gamma, \mathrm{V}_{0}, \rho_{0}, \mathrm{P}_{0}, \mathrm{~A}_{\text {disk }}$, and $\mathrm{P}_{\text {input }}$. With a total of seven variables and three physical dimensions, the four resulting dimensionless parameters are $\frac{\mathrm{P}_{\text {input }}}{\frac{1}{2} \rho_{0} \mathrm{~A}_{\mathrm{disk}} \mathrm{V}_{0}^{3}}, \mathrm{~V}_{0}\left(\gamma \cdot \frac{\mathrm{P}_{0}}{\rho_{0}}\right)^{-\frac{1}{2}}, \gamma$, and $\eta_{\text {prop }}$ of which the first and second are the power coefficient $C_{P}$ and the free-stream Mach number $M_{0}$. The power coefficient $C_{P}$ and the free-stream Mach number $M_{0}$ are made into independent variables while $\gamma$ is kept as a constant. As a result, for a given $\gamma, \rho_{0}, P_{0}$, and $A_{\text {disk }}$, the nineteen unknown variables are solved in Engineering Equation Solver (EES) in terms of $C_{P}$ for varying values of $M_{0}$. Due to the power addition by the propeller at the disk plane, the velocity immediately before the disk is greater than the free-stream velocity and thus it is expected that $M_{1}$ reaches 1 before $M_{0}$. To keep the scope of the thesis within the subsonic regime, the solutions are solved for increasing $C_{p}$, 
starting from 0.001, until the Mach number immediately before the actuator disk reaches one. Note that a $C_{P}$ of zero means that no power is added to the flow at the disk plane and the flow variables at the disk and in the slipstream are thus equal to those at the free-stream.

Lastly, the solutions are nondimensionalized by the disk area and the free-stream pressure, density, velocity, and Mach number to obtain the following dimensionless variables $\frac{\rho_{1}}{\rho_{0}}, \frac{\rho_{2}}{\rho_{0}}, \frac{P_{1}}{P_{0}}, \frac{P_{2}}{P_{0}}, \frac{V_{1}}{V_{0}}, \frac{V_{2}}{V_{0}}, \frac{V_{3}}{V_{0}}, \frac{M_{1}}{M_{0}}, \frac{M_{2}}{M_{0}}, \frac{M_{3}}{M_{0}}, \frac{A_{0}}{A_{\text {disk }}}$, and $\frac{A_{3}}{A_{\text {disk }}}$. The pressure-rise across the disk is also made dimensionless by the dynamic pressure associated with the free-stream velocity giving the pressure-rise coefficient $\frac{\mathrm{P}_{2}-\mathrm{P}_{1}}{\frac{1}{2} \rho_{0} \mathrm{~V}_{0}^{2}}$.

Due to the implicit nature of the compressible flow equations given in section 3.2.1, it is more effective to make the dimensional solutions obtained from EES into dimensionless parameters than it is to develop dimensionless representations of the flow variables. As a result, dimensional equations are presented but dimensionless solutions are presented in the next section.

\subsubsection{Results}

For comparison, both compressible- and incompressible-flow solutions are determined for the bare propeller for a disk area of $1 \mathrm{ft}^{2}$ and for the free-stream flow at sea level static conditions as given in Table 1. The compressible-flow solutions are obtained for $\mathrm{M}_{0}$ of $0.25,0.40,0.55$, and 0.70, while the incompressible-flow solutions are obtained for a free-stream velocity $\mathrm{V}_{0}$ equivalent to Mach 0.55 at sea level static conditions.

Table 2 gives important compressible- and incompressible-flow solutions of the bare propeller obtained at the maximum power coefficient before the flow is sonic at station 1 for various freestream Mach numbers $\mathrm{M}_{0}$.

Figure 17 shows the maximum power coefficient that can be specified before $M_{1}$ is one for various free-stream Mach numbers. It is observed that the maximum power coefficient that is 
required for sonic flow immediately before the disk decreases rapidly with increasing free-stream Mach number $\mathrm{M}_{0}$. The propulsive efficiency, the total thrust coefficient, the dimensionless mass flowrate, the ratios of the far upstream and downstream areas to the disk area, and the dimensionless flow variables immediately before and after the disk and at the far downstream are plotted against the power coefficient $C_{p}$ for $M_{0}$ of $0.25,0.40,0.55$, and 0.70 in Figure 18 to Figure 31. When applicable, the dimensionless incompressible-flow solutions for $\mathrm{V}_{0}$ equivalent to Mach 0.55 at sea level static conditions are included in the plots for comparison. Note that the point where the flow becomes sonic before the disk is denoted by the symbol " $\mathrm{X}$ " in all of the plots.

Table 1. Table of given free-stream pressure and density and the disk area.

\begin{tabular}{|l|c|}
\hline Given Parameters & Value \\
\hline$\gamma($ compressible $)$ & 1.4 \\
\hline $\mathrm{P}_{0}\left(\mathrm{lbf} / \mathrm{ft}^{2}\right)$ & 2166.8 \\
\hline$\rho_{0}\left(\mathrm{slugs} / \mathrm{ft}^{3}\right)$ & $2.329 \times 10^{-3}$ \\
\hline $\mathrm{A}_{\text {disk }}\left(\mathrm{ft}^{3}\right)$ & 1 \\
\hline
\end{tabular}

Table 2. Table of flow solutions of the bare propeller obtained at the maximum power coefficient before the flow is sonic at station 1 for various free-stream Mach numbers. Note that $*$ means incompressible flow values.

\begin{tabular}{|l|c|c|c|c|c|c|c|c|}
\hline $\mathrm{M}_{0}$ & 0.20 & 0.30 & 0.40 & 0.55 & 0.60 & 0.70 & 0.80 & 0.90 \\
\hline $\mathrm{C}_{\mathrm{P}}\left(\mathrm{M}_{1}=1\right)$ & 59.682 & 15.450 & 5.572 & 1.570 & 1.054 & 0.457 & 0.173 & 0.039 \\
\hline$\eta_{\text {prop }}$ & 0.357 & 0.509 & 0.641 & 0.800 & 0.843 & 0.913 & 0.962 & 0.991 \\
\hline$\eta_{\text {prop }} *$ & 0.352 & 0.504 & 0.638 & 0.799 & 0.842 & 0.913 & 0.962 & 0.991 \\
\hline $\mathrm{A}_{0} / \mathrm{A}_{\text {disk }}$ & 2.964 & 2.035 & 1.590 & 1.255 & 1.188 & 1.094 & 1.038 & 1.009 \\
\hline $\mathrm{A}_{0} / \mathrm{A}_{\text {disk }} *$ & 2.844 & 1.983 & 1.567 & 1.251 & 1.187 & 1.095 & 1.040 & 1.010 \\
\hline $\mathrm{A}_{3} / \mathrm{A}_{\text {disk }}$ & 0.645 & 0.694 & 0.749 & 0.836 & 0.865 & 0.919 & 0.961 & 0.990 \\
\hline $\mathrm{A}_{3} / \mathrm{A}_{\text {disk }} *$ & 0.607 & 0.669 & 0.734 & 0.833 & 0.864 & 0.920 & 0.963 & 0.991 \\
\hline $\mathrm{V}_{3} / \mathrm{V}_{0}$ & 4.598 & 2.931 & 2.122 & 1.500 & 1.374 & 1.191 & 1.080 & 1.019 \\
\hline $\mathrm{V}_{3} / \mathrm{V}_{0} *$ & 4.689 & 2.965 & 2.134 & 1.502 & 1.374 & 1.191 & 1.080 & 1.019 \\
\hline
\end{tabular}




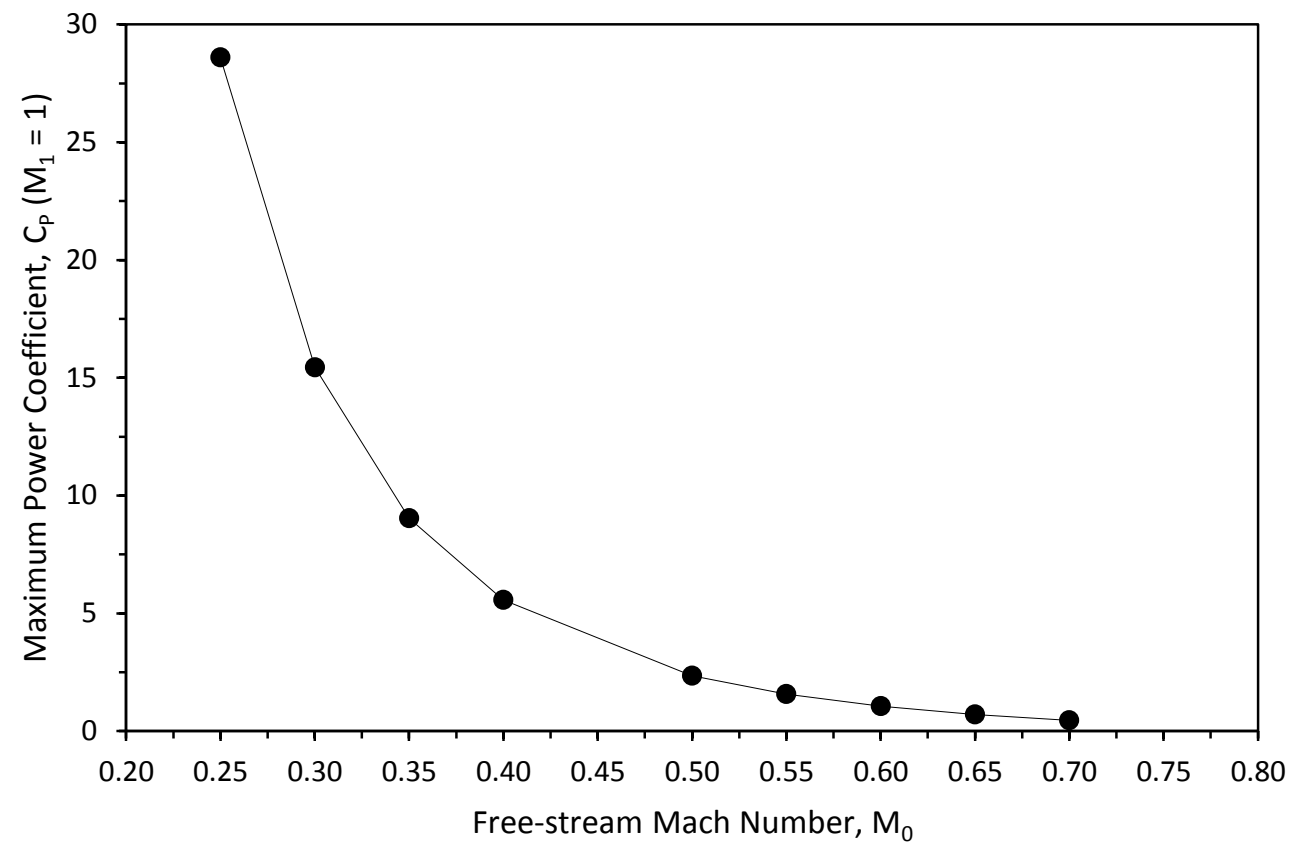

Figure 17. The plot gives the maximum power coefficient to drive the propeller before the flow is sonic at station 1 against the free-stream Mach number for the bare propeller.

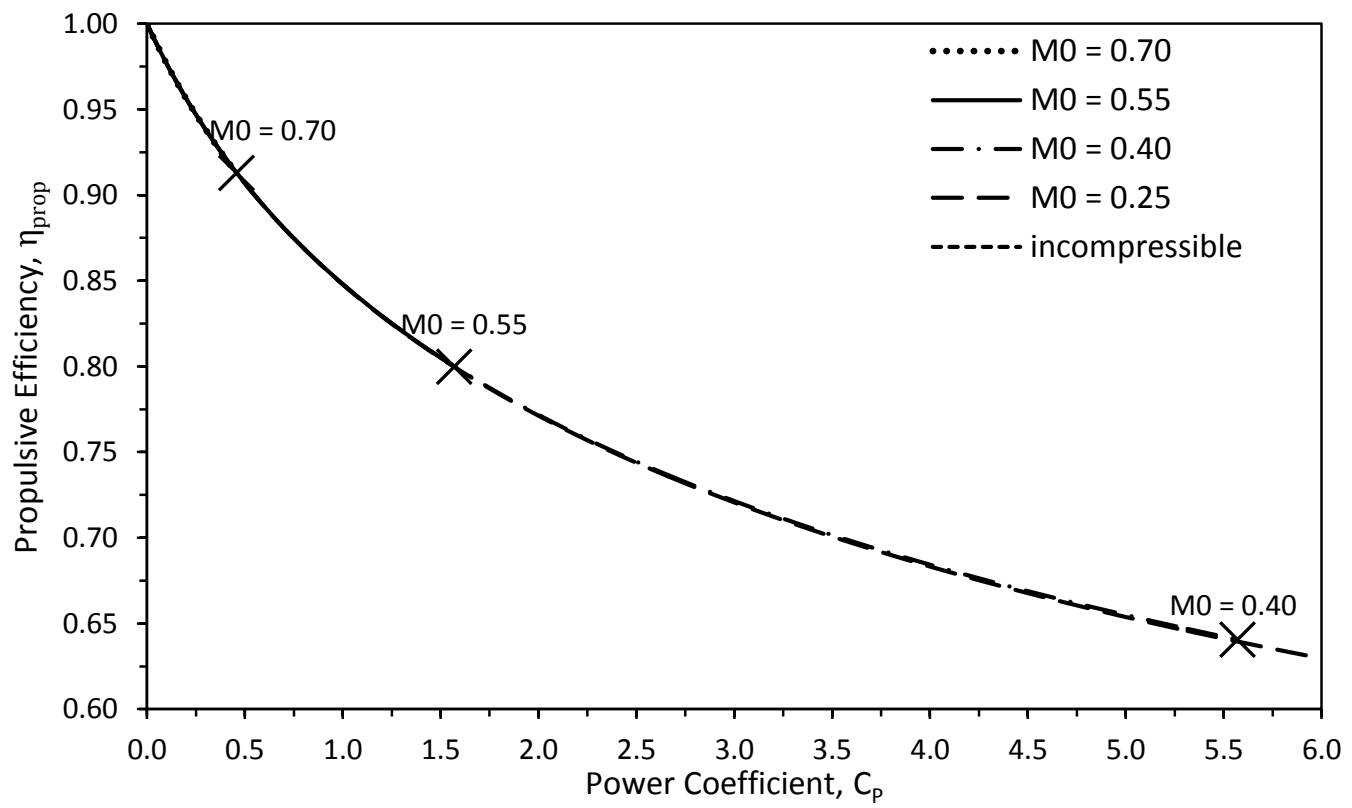

Figure 18. Propulsive efficiency of the bare propeller for incompressible flow and compressible flow of varying free-stream Mach numbers. 


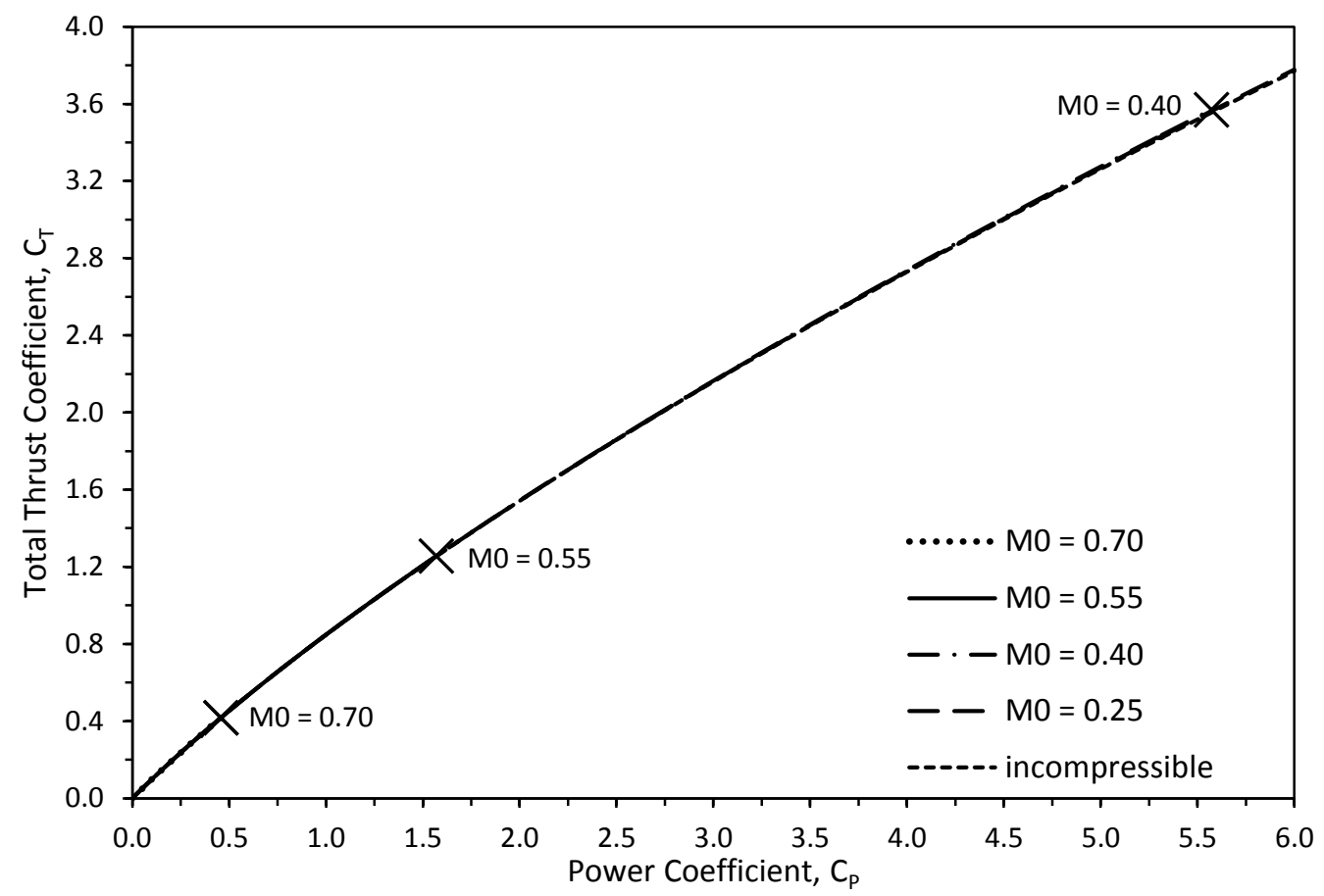

Figure 19. Comparison of the total thrust coefficient between incompressible flow and compressible flow of varying free-stream Mach numbers for the bare propeller.

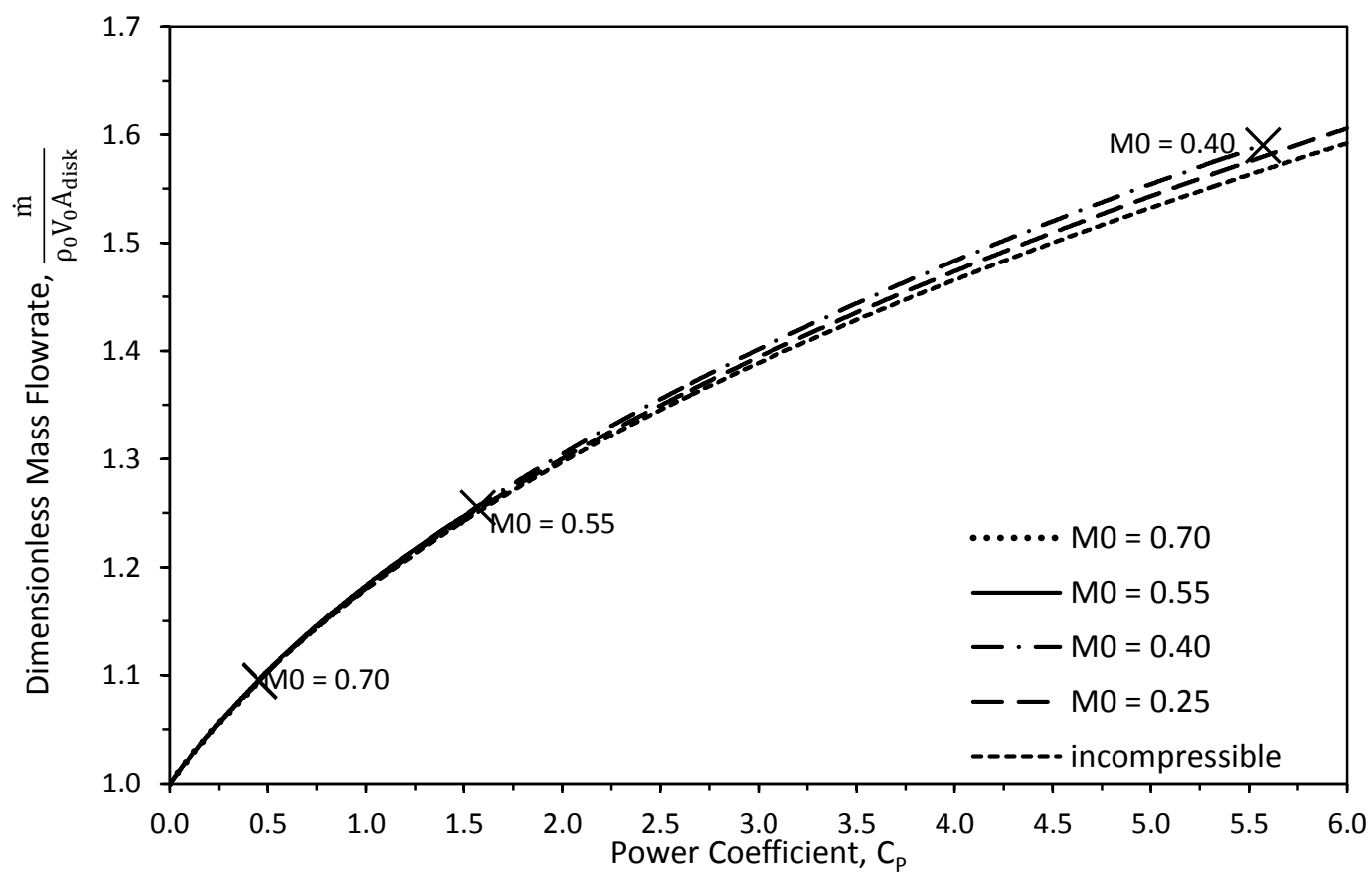

Figure 20. Comparison of the dimensionless mass flowrate between incompressible flow and compressible flow of varying free-stream Mach numbers for the bare propeller. 


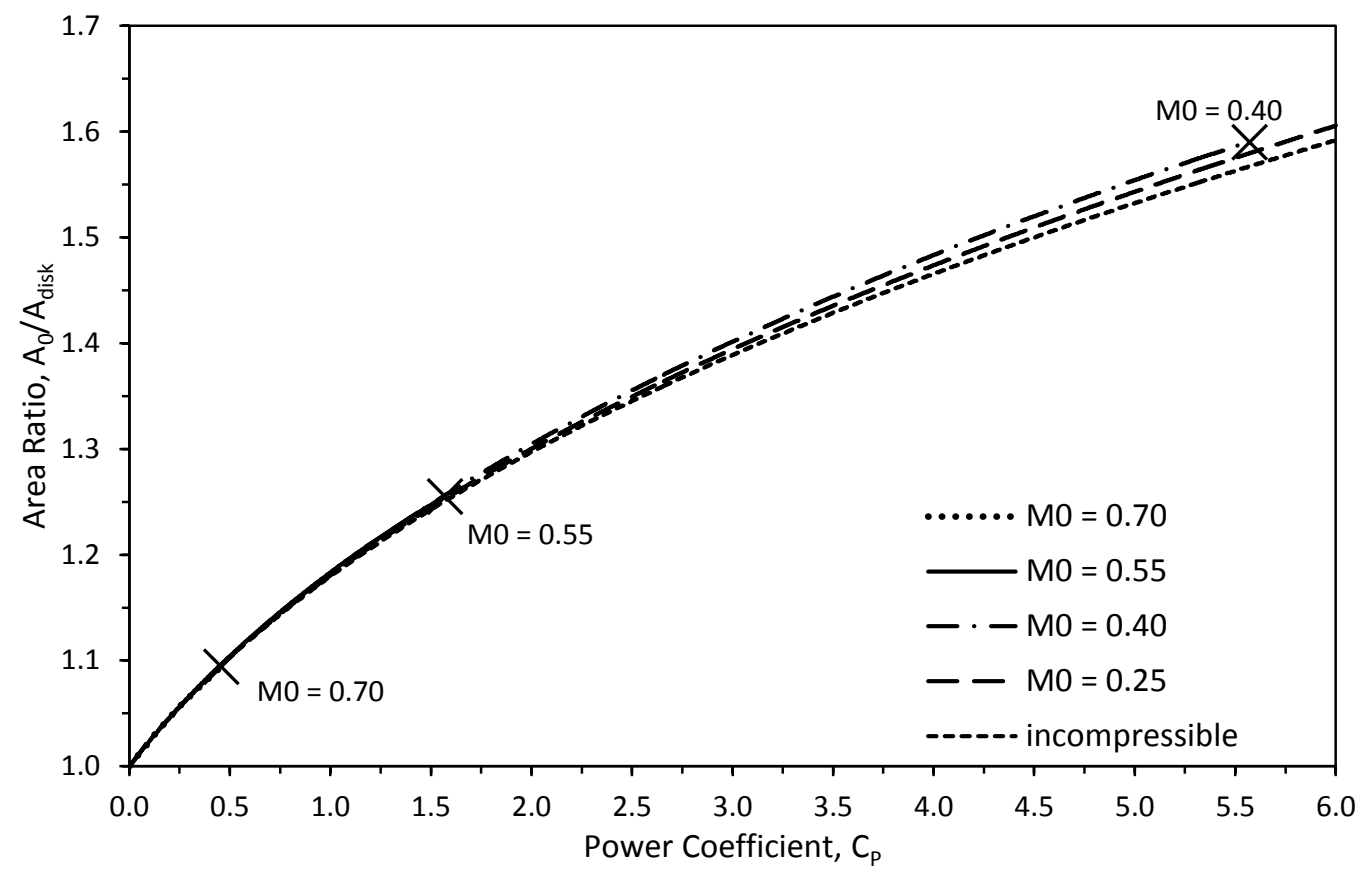

Figure 21. Comparison of the far upstream cross-sectional area between incompressible flow and compressible flow of varying free-stream Mach numbers for the bare propeller.

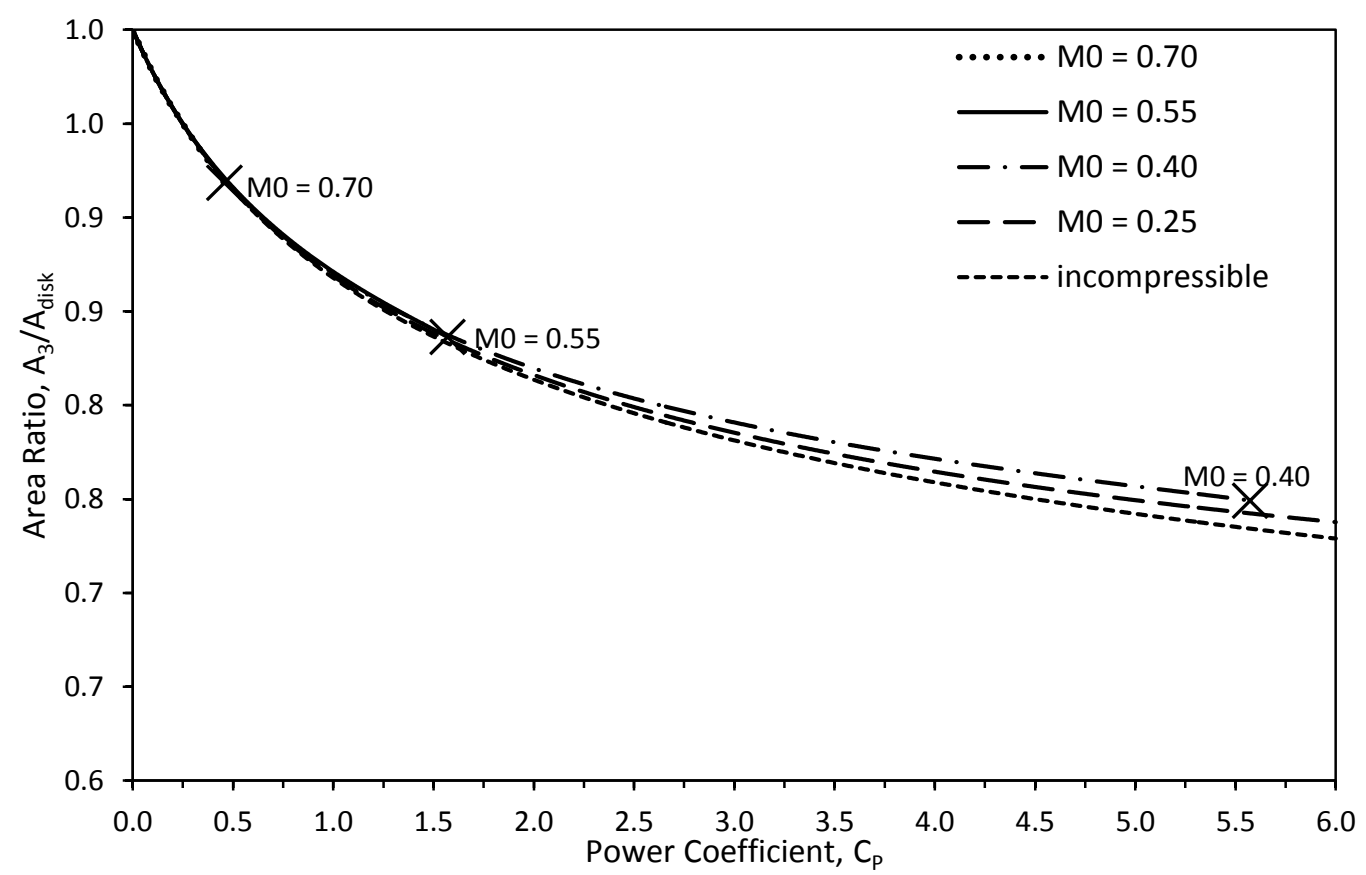

Figure 22. Comparison of the far downstream cross-sectional area between incompressible flow and compressible flow of varying free-stream Mach numbers for the bare propeller. 


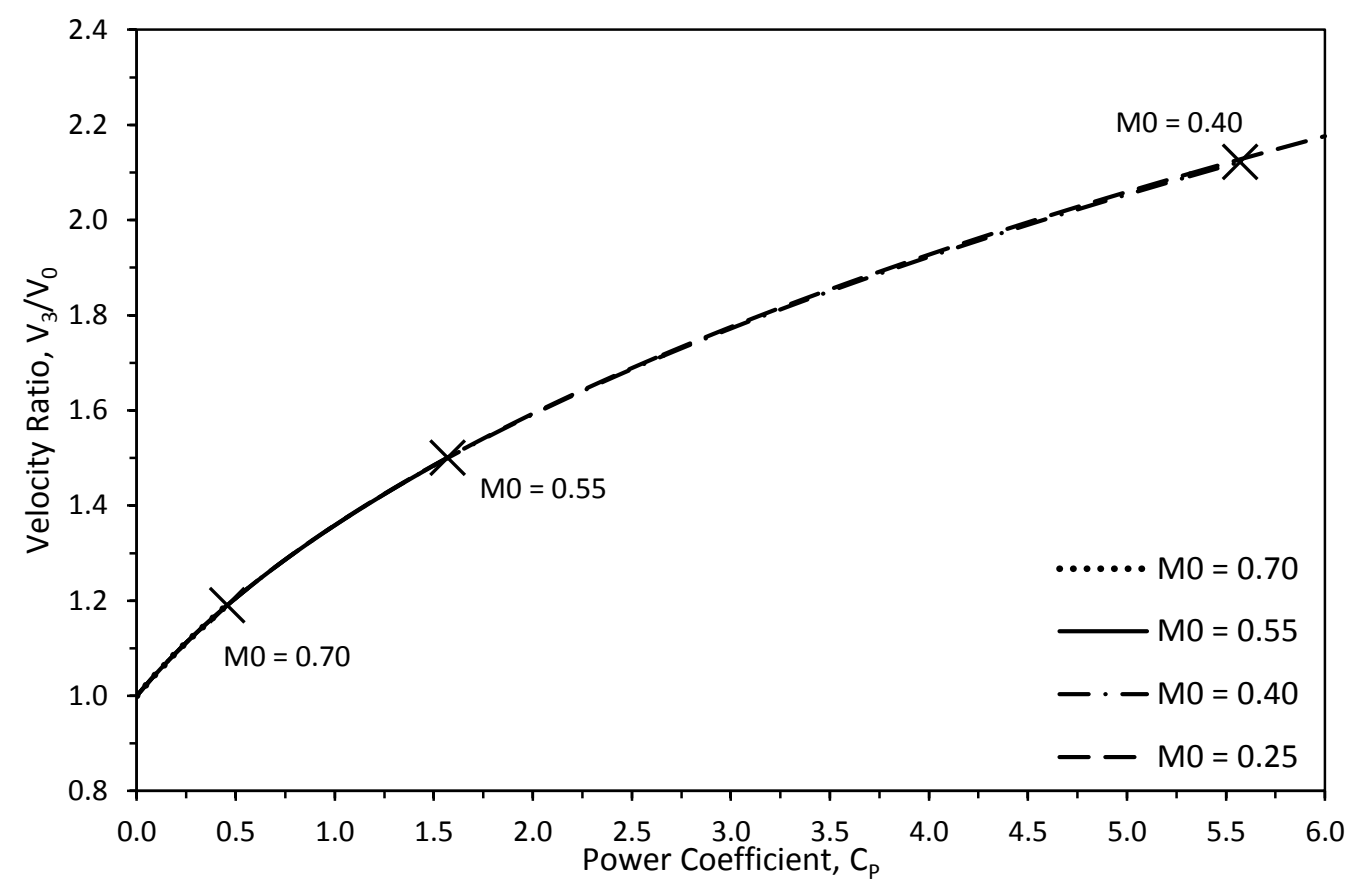

Figure 23. Comparison of the far downstream velocity between varying free-stream Mach numbers for the bare propeller.

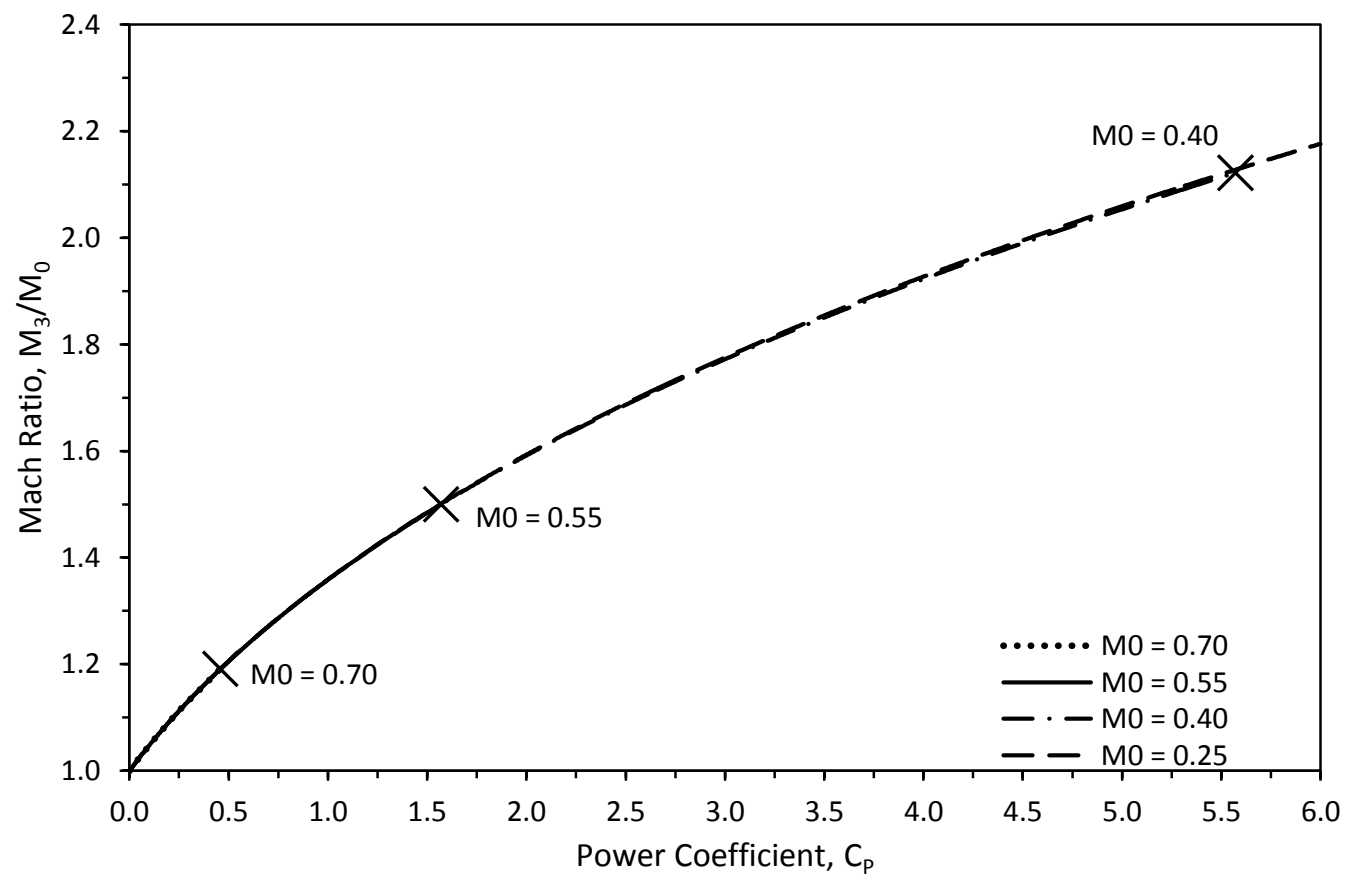

Figure 24. Comparison of the far downstream Mach number between varying free-stream Mach numbers for the bare propeller. 


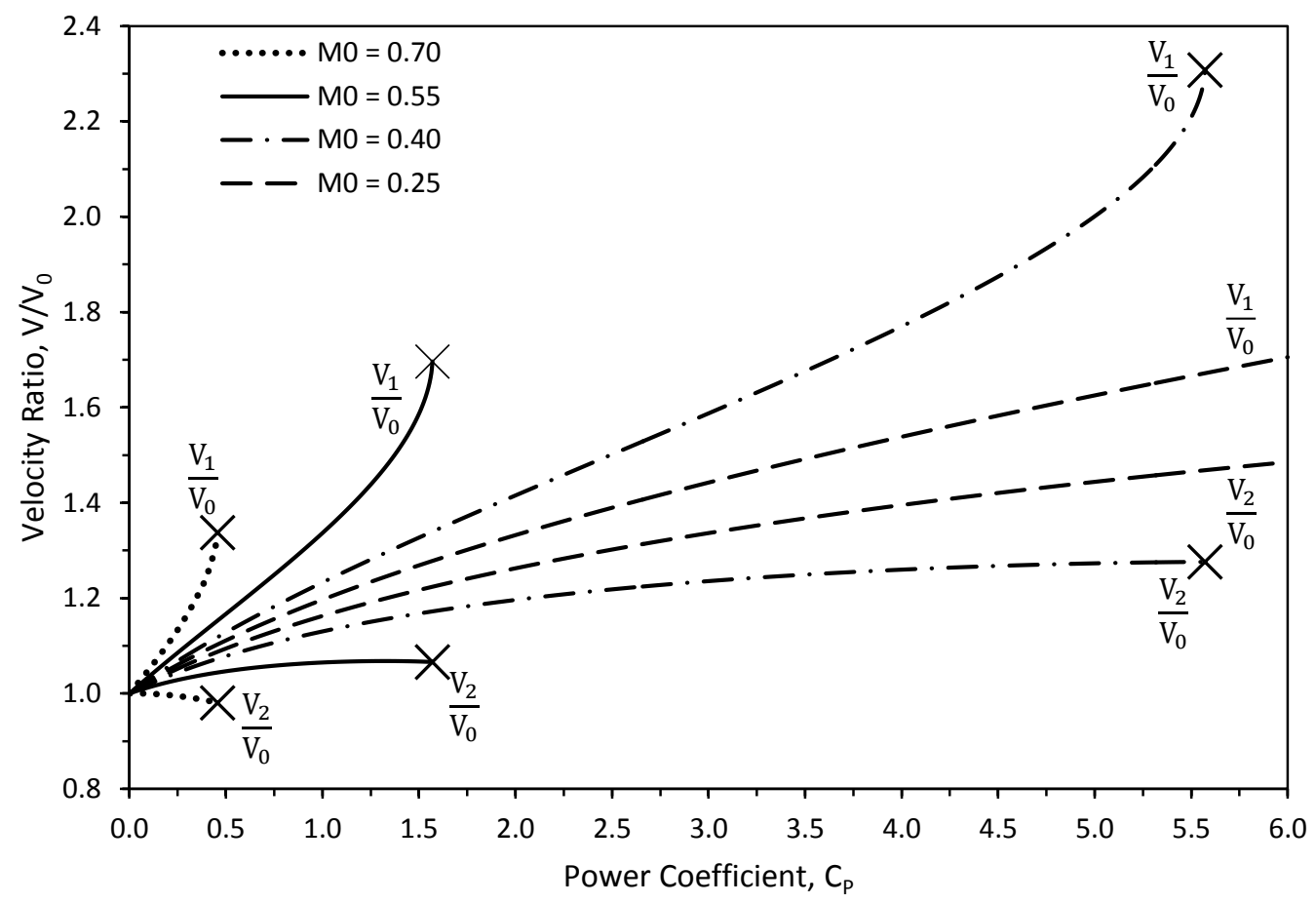

Figure 25. Comparison of the velocities immediately before and after the bare propeller for varying free-stream Mach numbers.

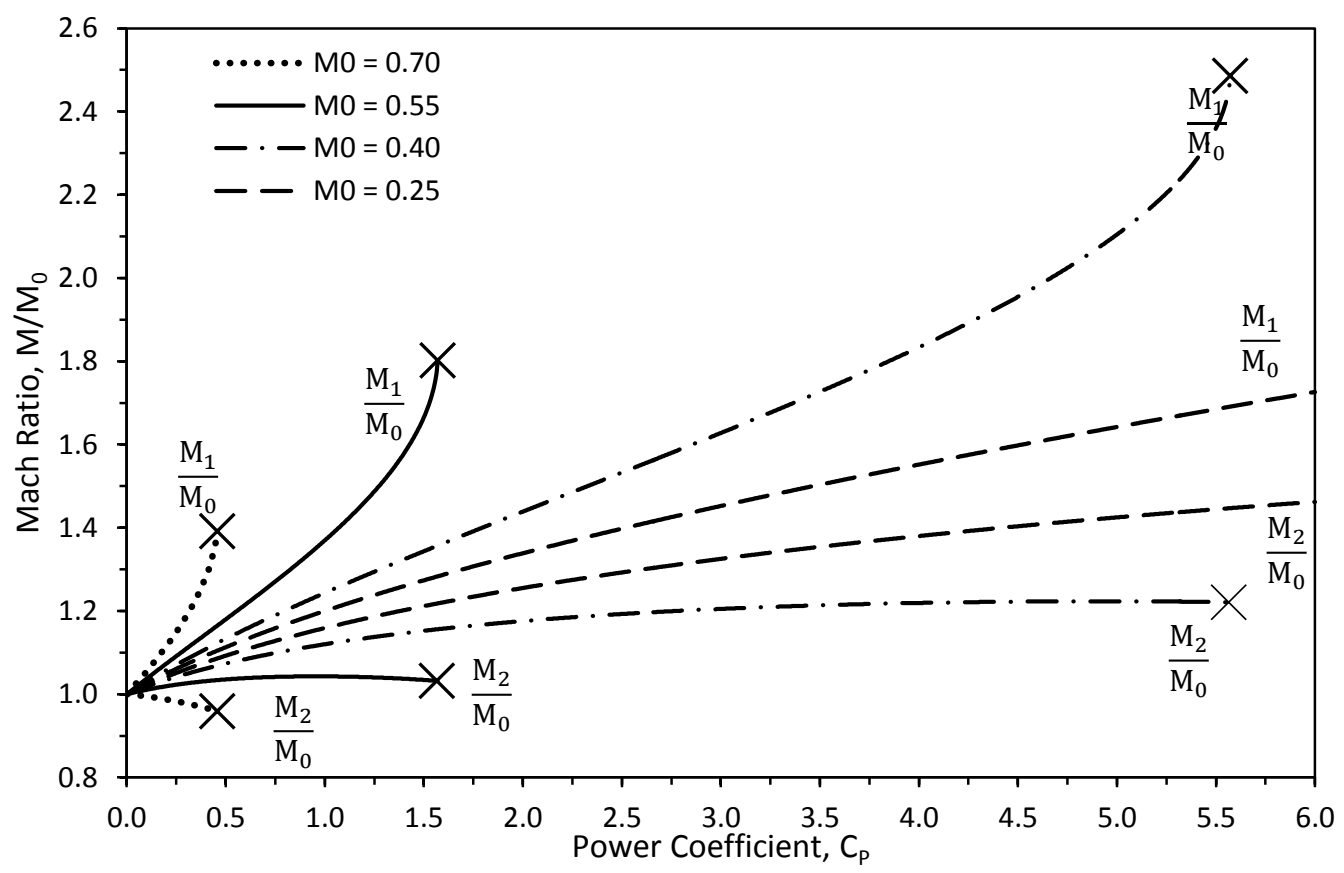

Figure 26. Comparison of the Mach numbers immediately before and after the bare propeller for varying free-stream Mach numbers. 


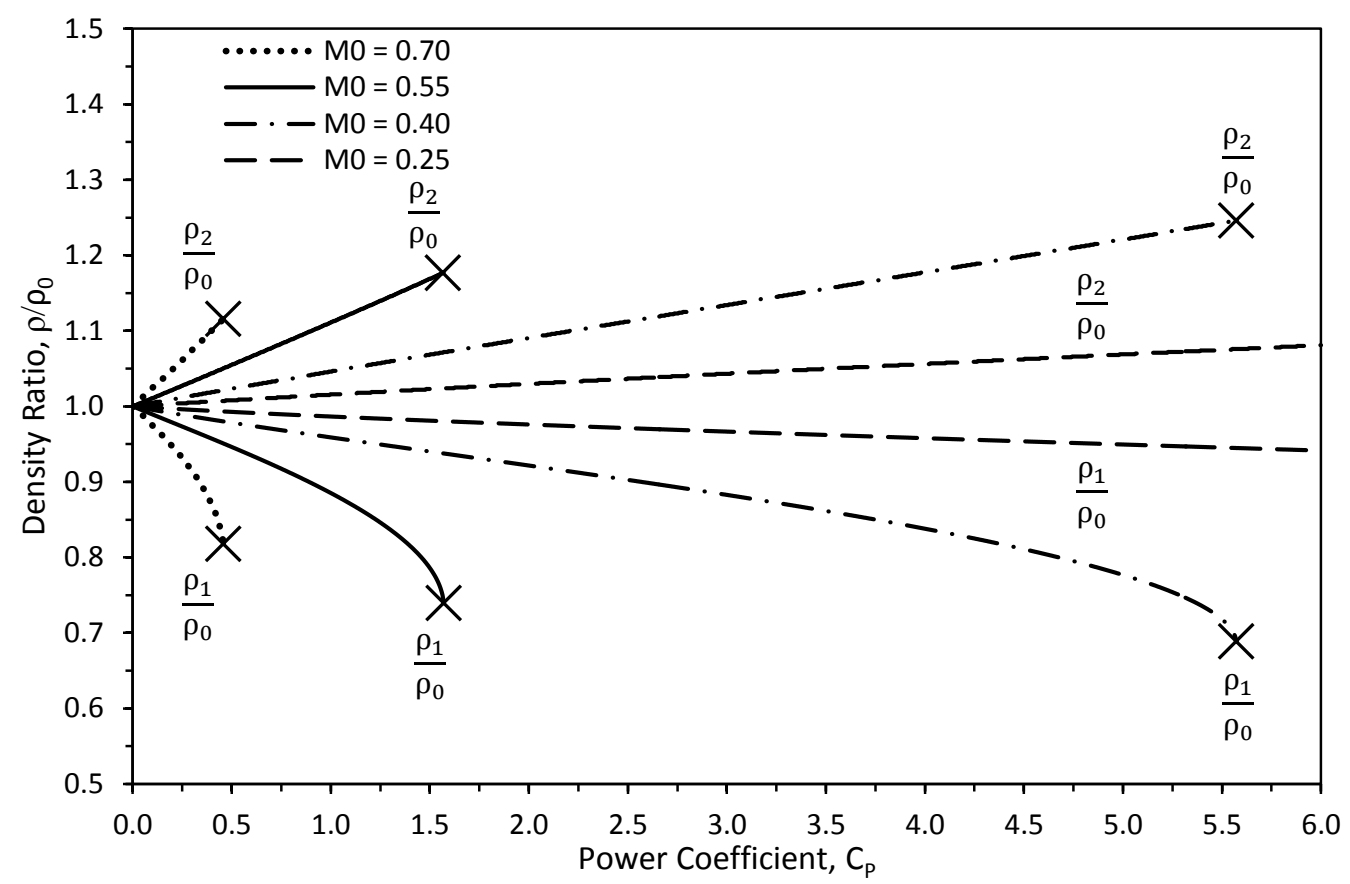

Figure 27. Comparison of the densities immediately before and after the bare propeller for varying free-stream Mach numbers.

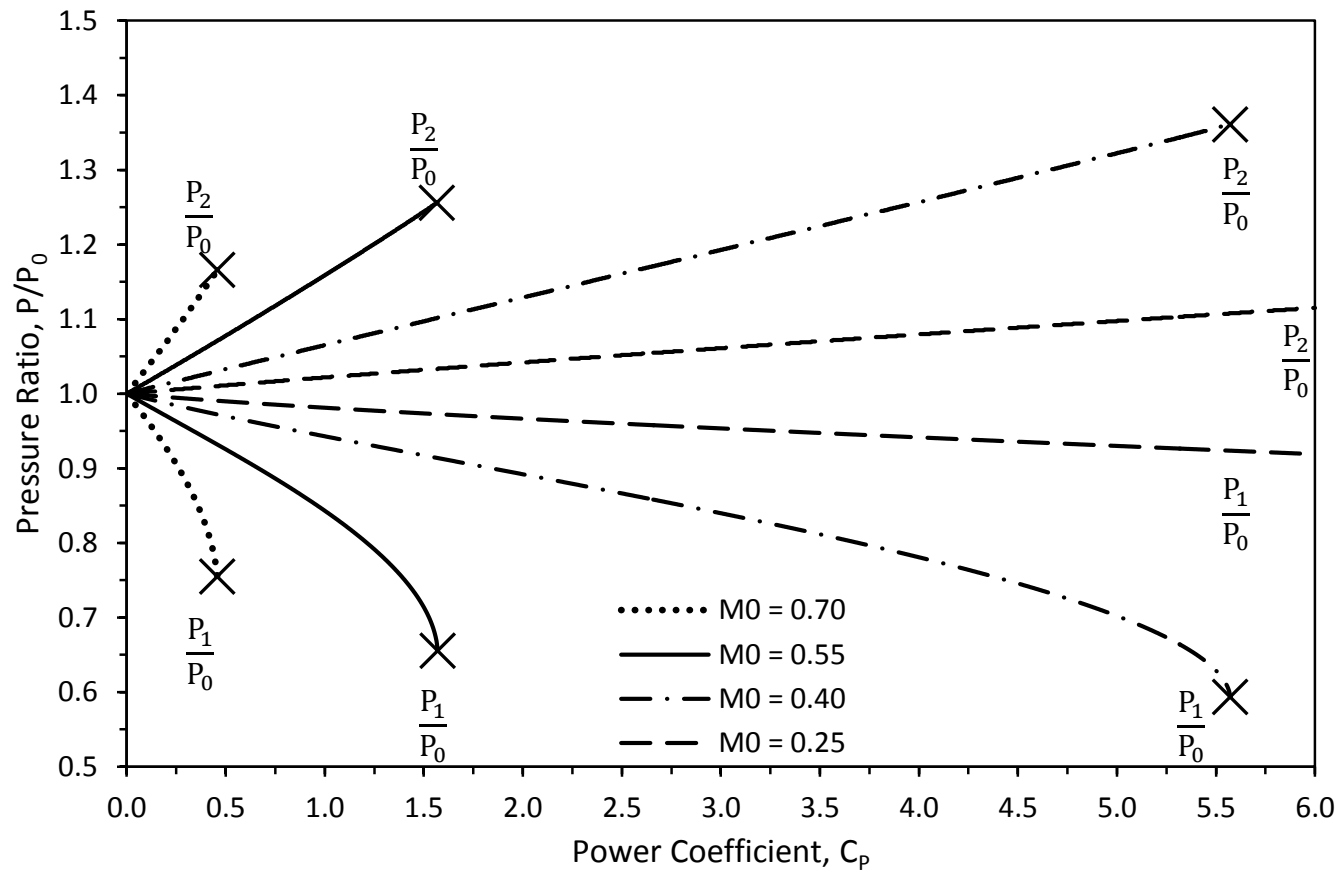

Figure 28. Comparison of the pressures immediately before and after the bare propeller for varying free-stream Mach numbers. 


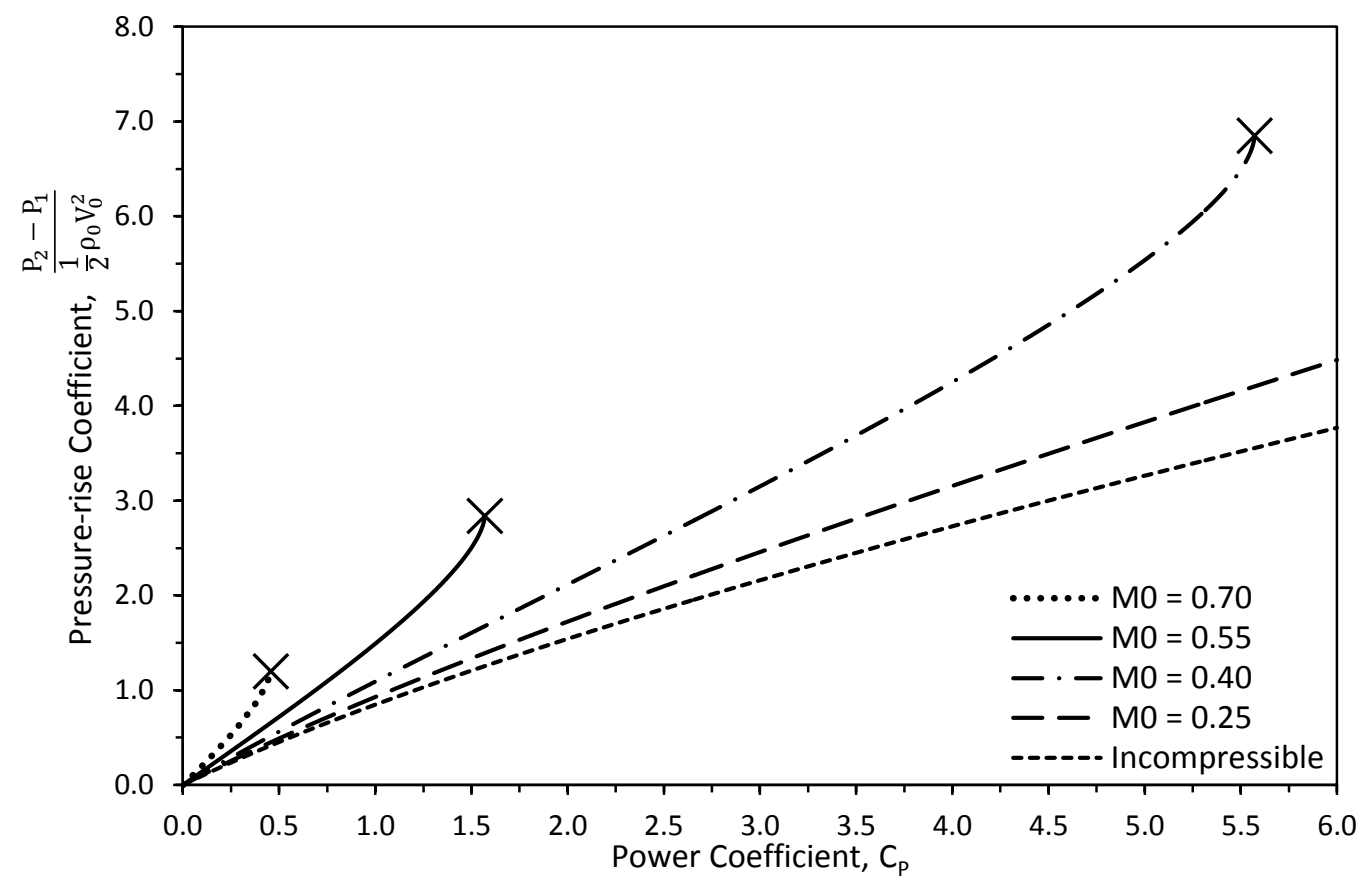

Figure 29. Comparison of the pressure rise across the bare propeller for incompressible flow and compressible flow of varying free-stream Mach numbers.

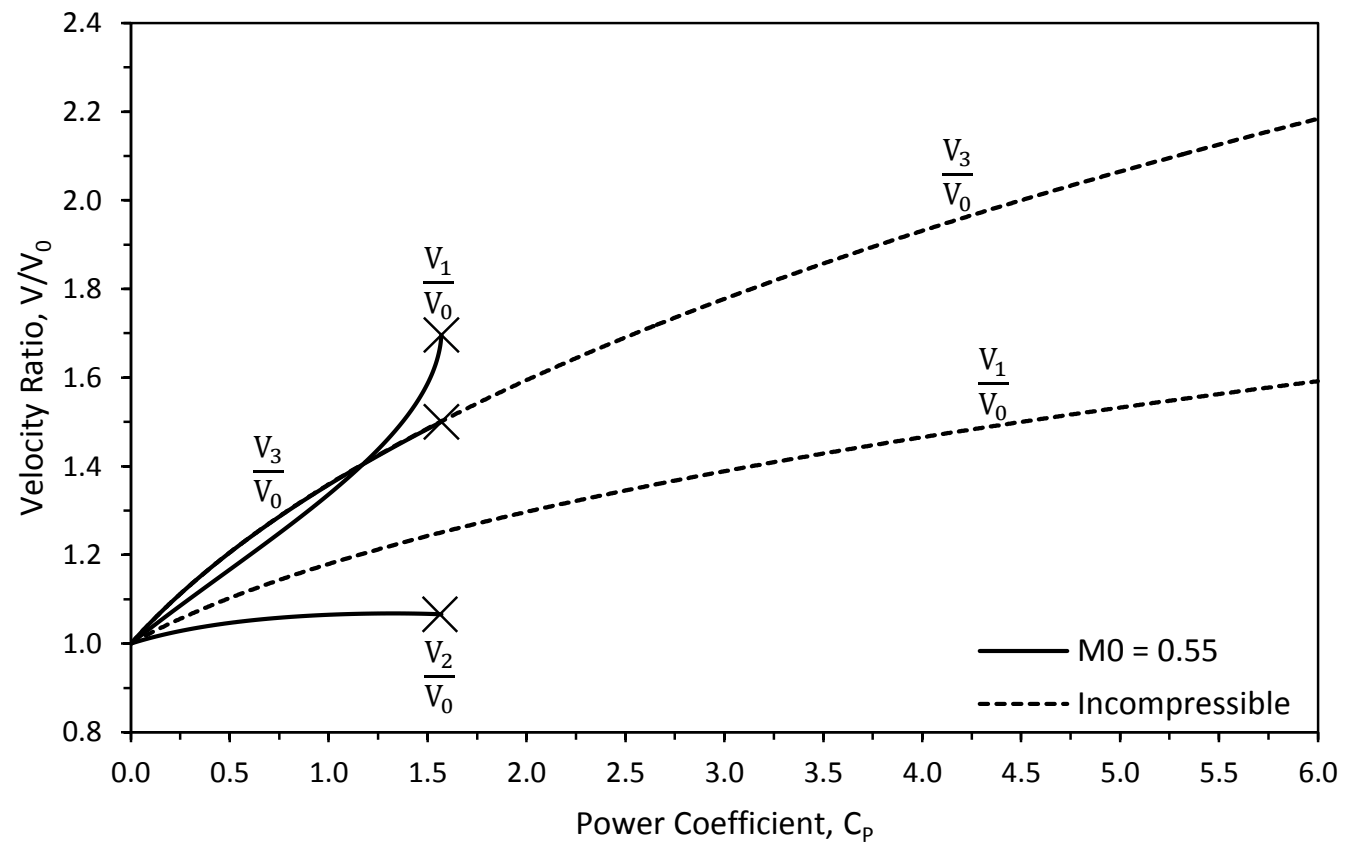

Figure 30. Comparison of the velocities throughout the streamtube between incompressible and compressible flow of the same free-stream velocity for the bare propeller. 


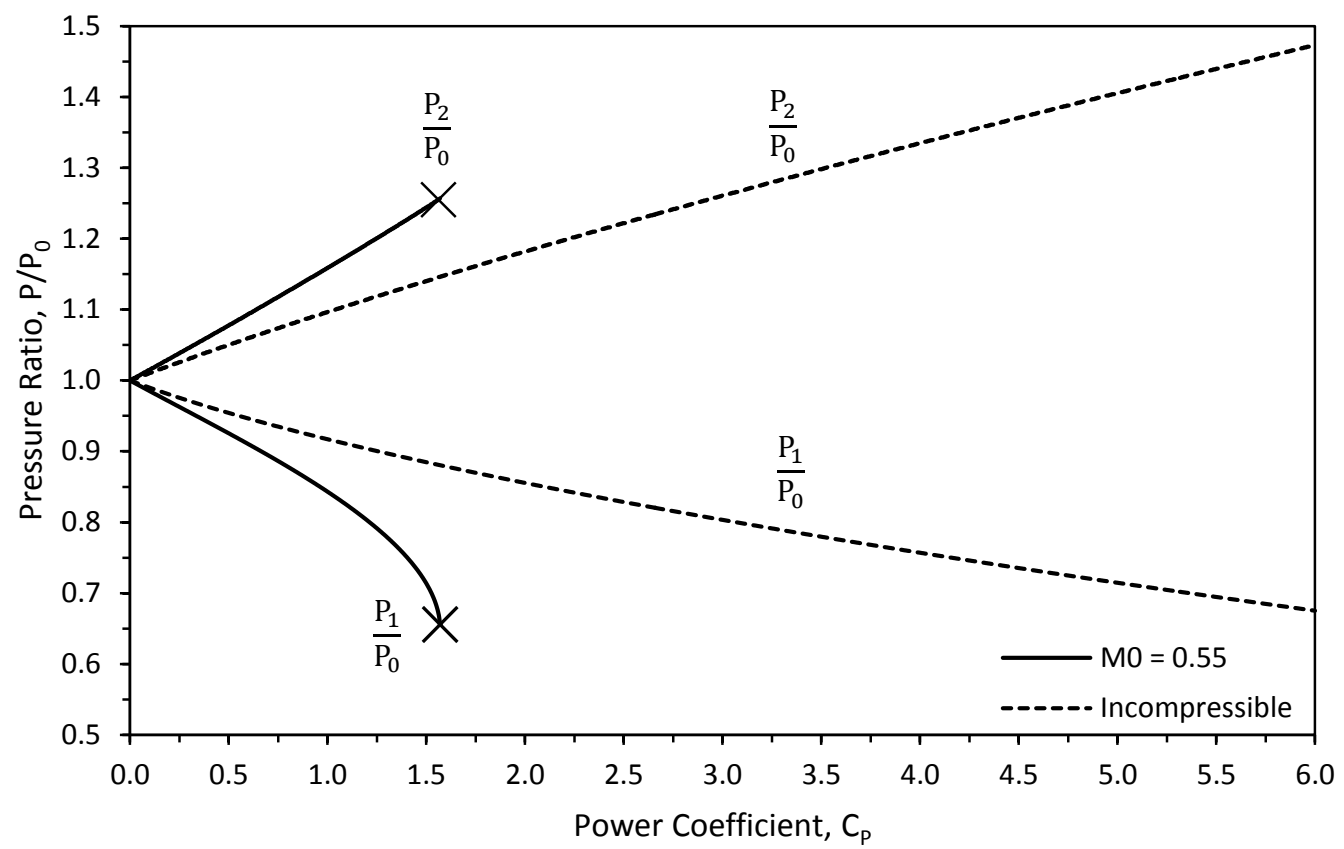

Figure 31. Comparison of the pressures immediately before and after the bare propeller between incompressible and compressible flow of the same free-stream velocity.

\subsubsection{Discussion}

It is concluded in the NACA report "Compressible-Flow Solutions for the Actuator Disk Theory" [5] that the isentropic propulsive efficiency of the bare propeller is the same for compressible and incompressible flow. It is found that the propulsive efficiency of the bare propeller does differ, although very slightly, between compressible and incompressible flow at very large power coefficients for low subsonic free-stream Mach numbers.

Table $\mathbf{2}$ gives the propulsive efficiency of both the compressible and incompressible bare propeller at the maximum power coefficient before the flow is sonic at station 1 for free-stream Mach numbers ranging from very low to very high subsonic values. As a check, these propulsive efficiencies are found to be consistent with the values obtained by the NACA report for $\mathrm{M}_{0}$ of $0.6,0.7,0.8,0.85$, and 0.9 . The propulsive efficiency of the bare propeller is observed to be approximately the same for compressible and incompressible flow for high subsonic free-stream 
Mach numbers $\mathrm{M}_{0}$. This is because at high free-stream Mach numbers the flow immediately before the disk becomes sonic at very low power coefficients that little to no change in the propulsive efficiency can be observed as shown in Figure 18. When looking at the case for $\mathrm{M}_{0}$ of 0.2 where an extremely large power coefficient is required to have sonic flow before the disk, a 0.005 increase in the propulsive efficiency between the compressible and incompressible flow is observed in

Table 2. As $M_{0}$ increases, the change in the efficiency practically goes to zero.

Since the propulsive efficiency is only dependent on the ratio of the far downstream and upstream velocities as seen in equation 3-29, and if the propulsive efficiency for the bare propeller is the same for compressible and incompressible flow, then logically the same conclusion can be made for the velocity ratio $\frac{V_{3}}{V_{0}}$. Furthermore, since the velocity ratio $\frac{V_{3}}{v_{0}}$ given in equation 3-26 only depends on the area ratio $\frac{A_{0}}{A_{\text {disk }}}$ for a given power coefficient, the same conclusion can also be said for $\frac{A_{0}}{A_{\text {disk }}}$. However,

Table 2 shows a 0.038 difference in $\frac{A_{0}}{A_{\text {disk }}}$ and a 0.091 difference in $\frac{V_{3}}{V_{0}}$ between compressible and incompressible flow for $\mathrm{M}_{0}$ of 0.2 at a maximum power coefficient of 58.7, showing that the propulsive efficiency do differ in the most extreme cases. This slight difference in $\frac{A_{0}}{A_{\text {disk }}}$ is visible in Figure 21 for $M_{0}$ of 0.40 and 0.25 and the difference increases with increasing $C_{P}$ up to the point where the flow before the disk is sonic. Again as a check, the values given in

Table 2 for $\frac{A_{0}}{A_{\text {disk }}}$ and $\frac{V_{3}}{V_{0}}$ are found to be consistent with the results in the NACA report for $M_{0}$ of $0.6,0.7,0.8,0.85$, and 0.9 .

The same trend can be seen throughout the total thrust coefficient, the dimensionless mass flowrate, the area ratio $\frac{A_{3}}{A_{\text {disk }}}$, the velocity ratio $\frac{V_{3}}{V_{0}}$, and the Mach ratio $\frac{M_{3}}{M_{0}}$ as shown in Figure 19 to 
Figure 24. The curves for these dimensionless variables appear to fall on top of each other up to the point where the flow becomes sonic before the disk. This is more evident in the total thrust coefficient, the velocity ratio $\frac{V_{3}}{V_{0}}$, and the Mach ratio $\frac{M_{3}}{M_{0}}$ where there is no deviation in the curves even at the lower free-stream Mach numbers such as 0.4 and 0.25 . However, a slight deviation can be observed for the dimensionless mass flowrate and the area ratio $\frac{A_{3}}{A_{\text {disk }}}$ at the lower Mach numbers.

Next, the flow variables across the bare propeller are compared for compressible flow between varying free-stream Mach numbers. Figure 25 to Figure 29 show that there is a rise in pressure and density but a drop in velocity and in turn the Mach number across the disk. The difference in the pressure, density, and the velocity across the disk increases with the power coefficient and is more prominent when the free-stream Mach number is higher. When comparing the solutions between the compressible and incompressible flow of the same free-stream velocity, the pressure rise across the disk is much greater for compressible flow than for incompressible flow while the incompressible disk velocity is between the values of the velocity immediately before and after the disk for compressible flow as observed Figure 30 and Figure 31. These results are consistent with the findings of Delano and Crigler [5]. 


\subsection{Compressible, Ducted Propeller}

This section will focus on the governing equations for the compressible, ducted propeller. The propeller is treated an actuator disk that is encased by a constant area duct having a crosssectional area of $A_{\text {disk }}$.

Note that the development of the performance parameters and the method of solution for the ducted propeller are similar if not the same to what was done for the bare propeller. Note that the power addition by the propeller $\mathrm{P}_{\text {input }}$, the total thrust $\mathrm{T}_{\text {total }}$, the propeller thrust $\mathrm{T}_{\text {prop }}$, the power coefficient, the total thrust coefficient, and the propulsive efficiency in equations 3-1 to 3-4 that were established for the bare propeller also apply to the ducted propeller. However, due to the addition of the duct to the propeller assembly, the total thrust experienced by the flow is now the sum of the thrust exerted by the propeller and the lip thrust exerted by the duct lip

$$
\mathrm{T}_{\text {total }}=\mathrm{T}_{\text {prop }}+\mathrm{T}_{\text {lip }}
$$

Next, the alternative forms of the power and thrust coefficient in equations 3-8 and 3-10 will be put only in terms of the velocity ratio $\frac{V_{3}}{V_{0}}$. By asserting that $A_{3}=A_{\text {disk }}$ and $\rho_{0}=\rho_{3}$ to the conservation of mass between station 0 and 3 ,

$$
\rho_{0} A_{0} V_{0}=\rho_{3} A_{3} V_{3}
$$

the ratio of the far upstream area to the disk area can be found

$$
\frac{A_{0}}{A_{\text {disk }}}=\frac{V_{3}}{V_{0}}
$$

By substituting equation 3-32 into 3-8 and 3-10, the power coefficient and the total thrust coefficient are now only in terms of $\frac{\mathrm{V}_{3}}{\mathrm{~V}_{0}}$

$$
\begin{gathered}
C_{p}=\frac{V_{3}}{V_{0}}\left(\left(\frac{V_{3}}{V_{0}}\right)^{2}-1\right) \\
C_{T}=2 \frac{V_{3}}{V_{0}}\left(\frac{V_{3}}{V_{0}}-1\right)
\end{gathered}
$$

This form of the power and total thrust coefficients are only for the ducted propeller since the relation between the area ratio and the velocity ratio in equation 3-32 is not true for the bare 
propeller. Note that this form of the power and the total thrust coefficients are also the same for the incompressible ducted propeller.

\subsubsection{Summary of Governing Equations}

The complete set of the governing equations for the compressible ducted propeller are summarized below.

$$
\begin{aligned}
& \mathrm{M}_{0}=\mathrm{V}_{0}\left(\gamma \cdot \frac{\mathrm{P}_{0}}{\rho_{0}}\right)^{-\frac{1}{2}} \\
& \frac{A_{\text {disk }}}{A_{0}}=\frac{M_{0}}{M_{1}}\left(\frac{1+\frac{\gamma-1}{2} M_{1}^{2}}{1+\frac{\gamma-1}{2} M_{0}^{2}}\right)^{\frac{\gamma+1}{2(\gamma-1)}} \\
& \frac{\mathrm{P}_{1}}{\mathrm{P}_{0}}=\left(\frac{1+\frac{\gamma-1}{2} \mathrm{M}_{0}^{2}}{1+\frac{\gamma-1}{2} \mathrm{M}_{1}^{2}}\right)^{\frac{\gamma}{\gamma-1}} \\
& \frac{\rho_{1}}{\rho_{0}}=\left(\frac{\mathrm{P}_{1}}{\mathrm{P}_{0}}\right)^{\frac{1}{\gamma}} \\
& \frac{\mathrm{V}_{1}}{\mathrm{~V}_{0}}=\frac{\rho_{0}}{\rho_{1}} \frac{\mathrm{A}_{0}}{\mathrm{~A}_{\mathrm{disk}}} \\
& M_{3}=V_{3}\left(\gamma \cdot \frac{P_{0}}{\rho_{0}}\right)^{-\frac{1}{2}} \\
& \dot{\mathrm{m}}=\rho_{1} \mathrm{~A}_{\text {disk }} \mathrm{V}_{1} \\
& \mathrm{~T}_{\text {total }}=\dot{\mathrm{m}}\left(\mathrm{V}_{3}-\mathrm{V}_{0}\right) \\
& \mathrm{T}_{\text {prop }}=\dot{\mathrm{m}}\left(\mathrm{V}_{2}-\mathrm{V}_{1}\right)+\mathrm{A}_{\text {disk }}\left(\mathrm{P}_{2}-\mathrm{P}_{1}\right) \\
& \mathrm{T}_{\text {lip }}=\mathrm{T}_{\text {total }}-\mathrm{T}_{\text {prop }} \\
& \frac{\mathrm{T}_{\text {lip }}}{\frac{1}{2} \rho_{0} \mathrm{~V}_{0}^{2} \mathrm{~A}_{\text {disk }}}=2\left(\frac{\mathrm{V}_{1}}{\mathrm{~V}_{0}}\right)\left(\frac{\mathrm{V}_{1}}{\mathrm{~V}_{0}}-1\right)\left(1+\frac{\gamma-1}{2} \mathrm{M}_{0}^{2}\left(1-\left(\frac{\mathrm{V}_{1}}{\mathrm{~V}_{0}}\right)^{2}\right)\right)^{\frac{1}{\gamma-1}} \\
& +\frac{2}{\gamma \mathrm{M}_{0}^{2}}\left(\left(1+\frac{\gamma-1}{2} \mathrm{M}_{0}^{2}\left(1-\left(\frac{\mathrm{V}_{1}}{\mathrm{~V}_{0}}\right)^{2}\right)\right)^{\frac{\gamma}{\gamma-1}}-1\right) \\
& \mathrm{C}_{\mathrm{p}}=\frac{\mathrm{V}_{3}}{\mathrm{~V}_{0}}\left(\left(\frac{\mathrm{V}_{3}}{\mathrm{~V}_{0}}\right)^{2}-1\right)
\end{aligned}
$$




$$
\begin{gathered}
\mathrm{P}_{\text {input }}=\dot{\mathrm{m}} \frac{\mathrm{V}_{3}^{2}-\mathrm{V}_{0}^{2}}{2} \\
\mathrm{C}_{\mathrm{T}}=2 \frac{\mathrm{V}_{3}}{\mathrm{~V}_{0}}\left(\frac{\mathrm{V}_{3}}{\mathrm{~V}_{0}}-1\right) \\
\eta_{\text {prop }}=\frac{2}{\frac{\mathrm{V}_{3}}{\mathrm{~V}_{0}}+1}
\end{gathered}
$$

As stated previously for the bare propeller, these governing equations provide a system of equations to determine the flow variables before the actuator disk and throughout the slipstream and the performance of the ducted propeller in terms of the free-stream flow variables $\gamma, \rho_{0}, \mathrm{P}_{0}$, and $\mathrm{V}_{0}$ and the disk area $\mathrm{A}_{\text {disk }}$.

\subsubsection{Method of Solution}

The method of solution for the ducted propeller is the same as for the bare propeller. The power addition by the propeller to the flow $P_{\text {input }}$ is specified, along with the disk area $A_{\text {disk }}$, and the following free-stream flow variables $\gamma, \rho_{0}, \mathrm{P}_{0}$, and $\mathrm{V}_{0}$. With these six inputs, there are a total of fifteen unknown variables left to determine, which are the following flow variables $A_{0}, M_{0}, M_{1}$, $\rho_{1}, P_{1}, V_{1}, M_{3}$, and $V_{3}$ and the following performance parameters of the ducted propeller $T_{\text {disk}}$, $\mathrm{T}_{\text {total }}, \mathrm{T}_{\text {lip }}, \mathrm{C}_{\mathrm{T}}, \mathrm{C}_{\mathrm{P}}, \eta_{\text {prop }}$, and $\dot{\mathrm{m}}$. These fifteen unknown variables are solved in Engineering Equation Solver (EES) for the same $\gamma, \rho_{0}, P_{0}$, and $A_{d i s k}$ in terms of the power coefficient $C_{P}$ for varying values of $\mathrm{M}_{0}$ using the fifteen non-linear governing equations summarized in section 3.3.1. Beginning from 0.001 , the power coefficient $C_{P}$ is increased until the flow before the actuator disk becomes sonic for each value of $\mathrm{M}_{0}$.

The solutions are nondimensionlized by the given free-stream flow variables and the disk area giving $\frac{\rho_{1}}{\rho_{0}}, \frac{\mathrm{P}_{1}}{\mathrm{P}_{0}}, \frac{\mathrm{V}_{1}}{\mathrm{~V}_{0}}, \frac{\mathrm{M}_{1}}{\mathrm{M}_{0}}, \frac{\mathrm{A}_{0}}{\mathrm{~A}_{\text {disk }}}, \frac{\dot{\mathrm{m}}}{\rho_{0} \mathrm{~V}_{0} \mathrm{~A}_{\text {disk }}}$, and $\frac{\mathrm{P}_{2}-\mathrm{P}_{1}}{\frac{1}{2} \rho_{0} \mathrm{~V}_{0}^{2}}$. The lip thrust and the propeller thrust are also made dimensionless by the reference force to give the lip thrust and propeller thrust coefficients, 
$\frac{T_{\text {lip }}}{\frac{1}{2} \rho_{0} A_{\text {disk }} V_{0}^{2}}$ and $\frac{T_{\text {prop }}}{\frac{1}{2} \rho_{0} A_{\text {disk }} V_{0}^{2}}$, respectively. The dimensionless solutions are represented against the power coefficient $C_{P}$ for varying values of $M_{0}$ in the following section.

\subsubsection{Results}

The solutions to the ducted propeller are determined for the same free-stream flow conditions and disk area as the bare propeller given in Table 1. Compressible-flow solutions are obtained for the free-stream Mach number of $0.25,0.40,0.55$, and 0.7, while incompressible-flow solutions are obtained for the free-stream velocity equal to Mach 0.55 at sea level static conditions for comparison. Figure 32 shows the maximum power coefficient required to obtain sonic flow immediately before the disk plotted against the free-stream Mach number $M_{0}$. Other dimensionless parameters are plotted against the power coefficient in Figure 33 to Figure 43.

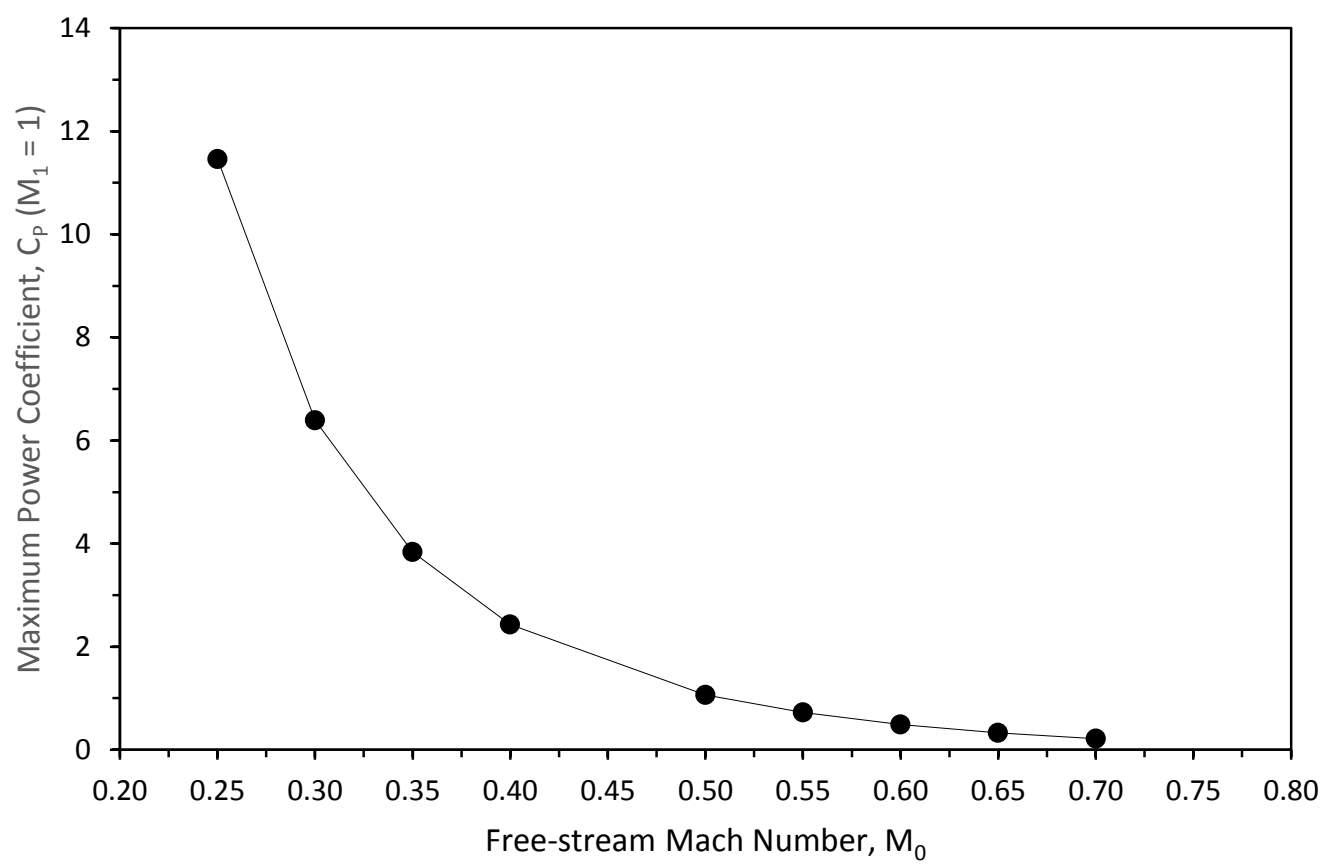

Figure 32. The plot gives the maximum power coefficient to drive the propeller before the flow is sonic at station 1 against the free-stream Mach number for the ducted propeller. 


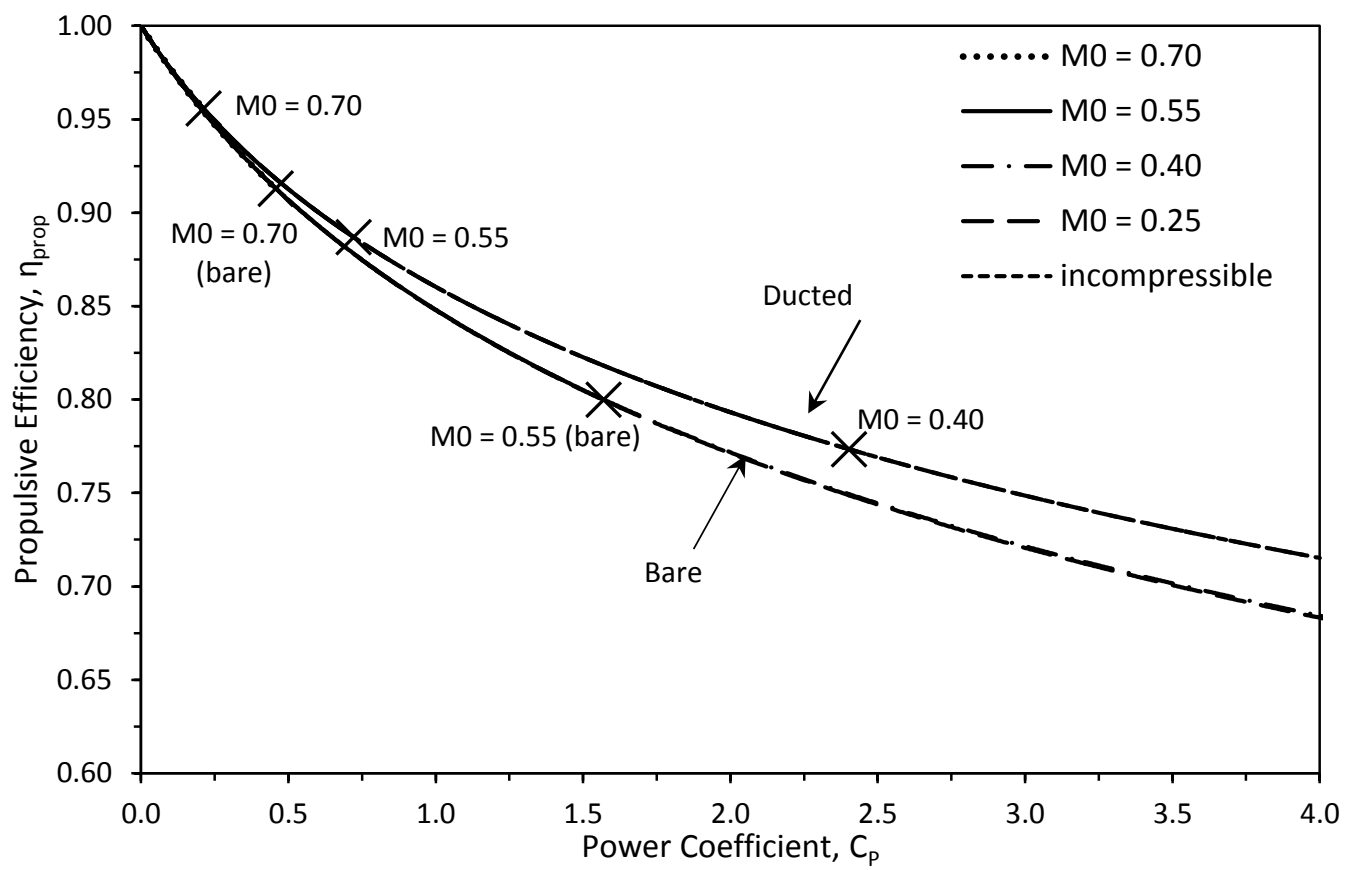

Figure 33. Propulsive efficiency of the bare and ducted propeller for incompressible flow and compressible flow of varying free-stream Mach numbers.

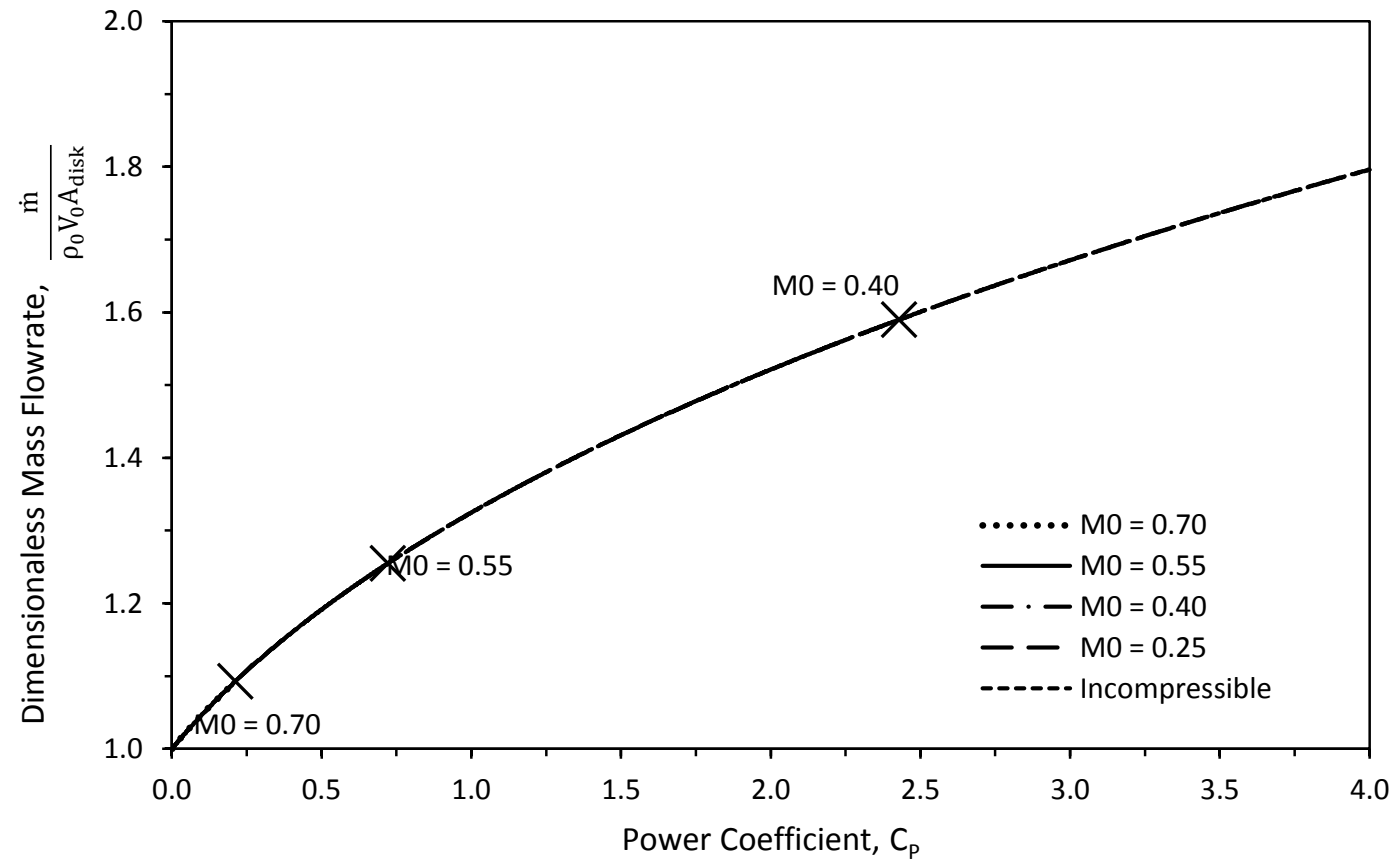

Figure 34. Comparison of the dimensionless mass flowrate between incompressible flow and compressible flow of varying free-stream Mach numbers for the ducted propeller. 


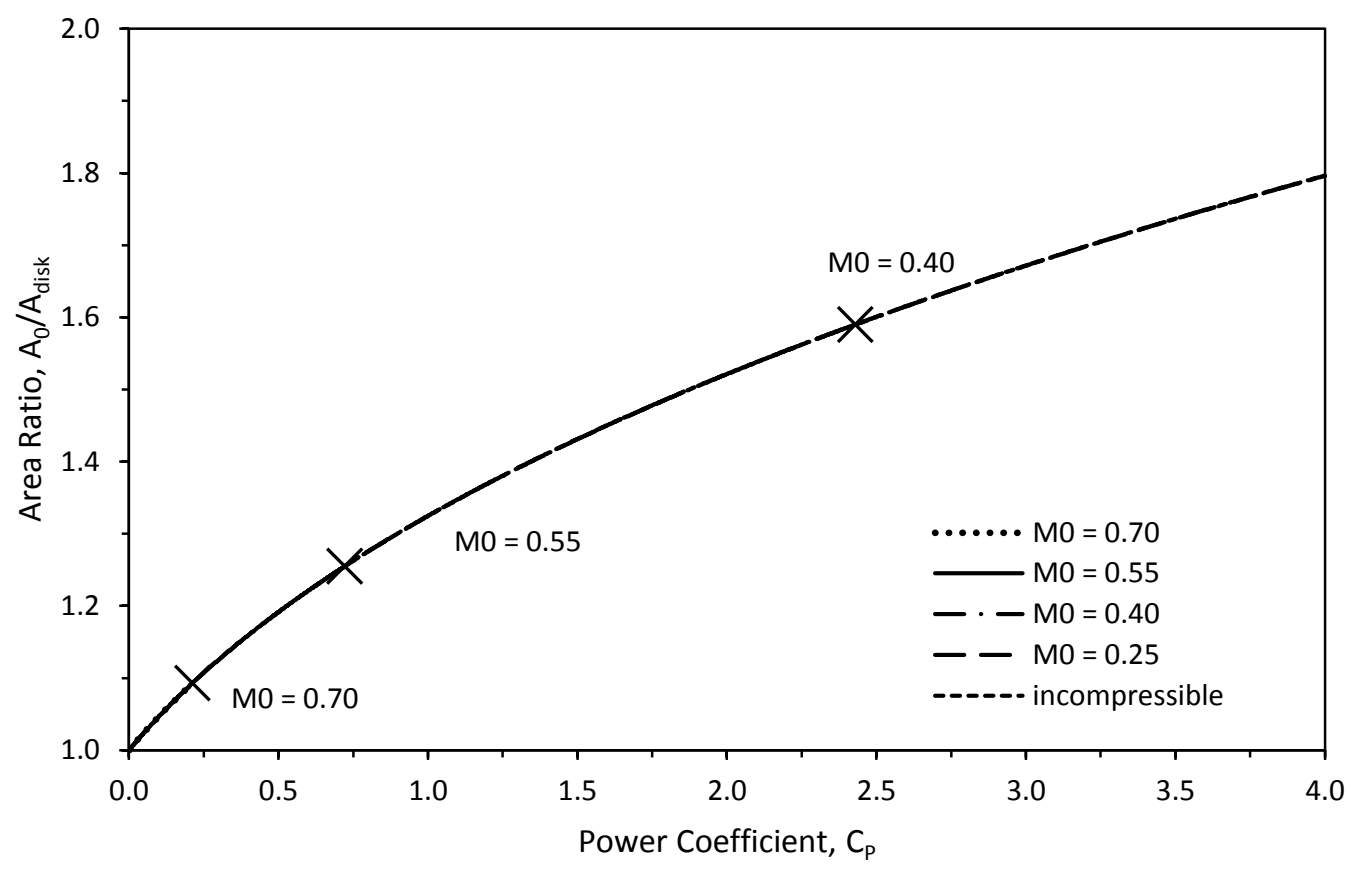

Figure 35. Comparison of the far upstream cross-sectional area for incompressible flow and compressible flow of varying free-stream Mach numbers for the ducted propeller.

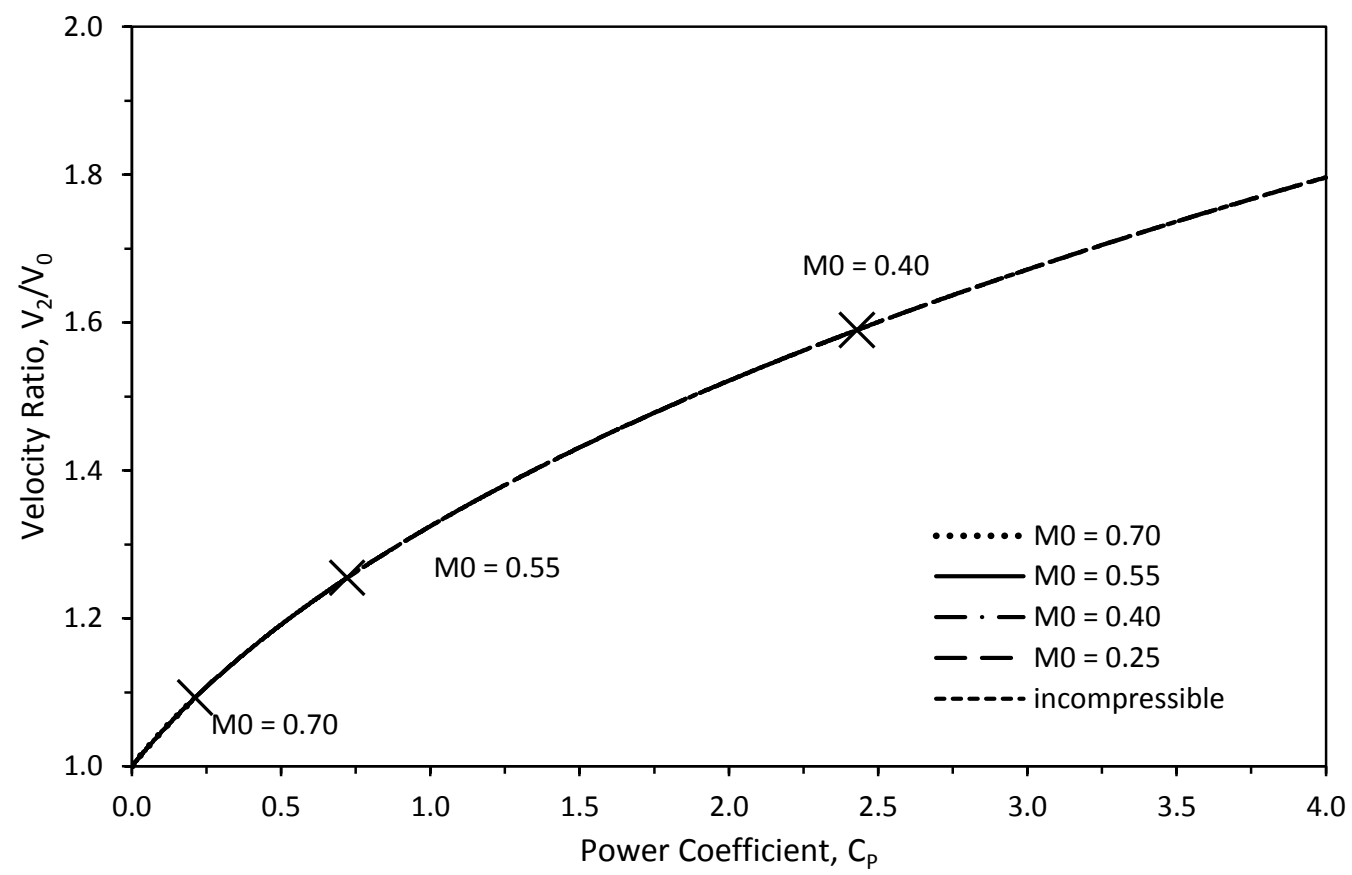

Figure 36. Comparison of the velocity immediately after the ducted propeller for incompressible flow and compressible flow of varying free-stream Mach numbers. Note that $\mathbf{V}_{\mathbf{2}}=\mathbf{V}_{\mathbf{3}}$. 


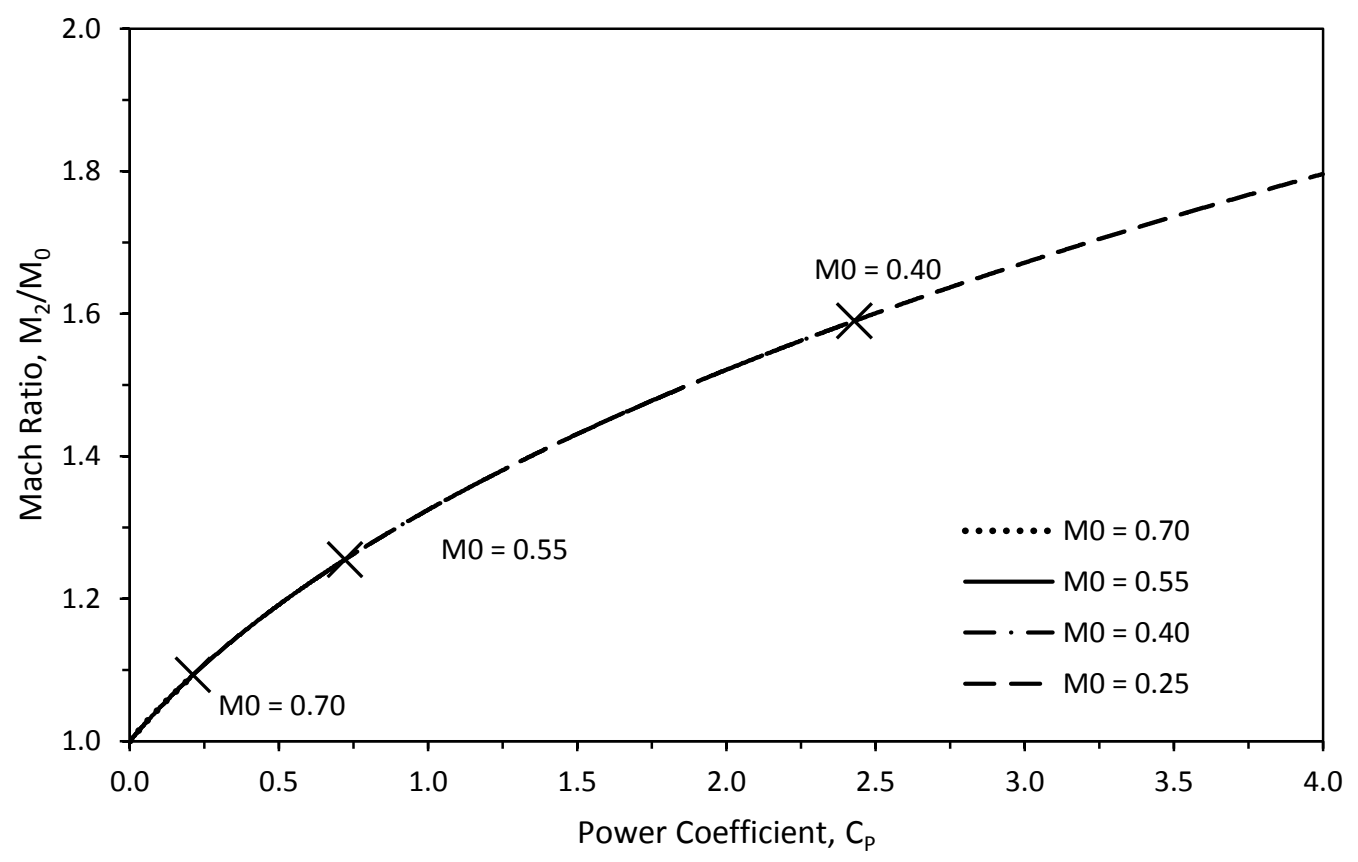

Figure 37. Comparison of the Mach number immediately after the ducted propeller for varying free-stream Mach numbers. Note that $\mathbf{M}_{2}=\mathbf{M}_{3}$.

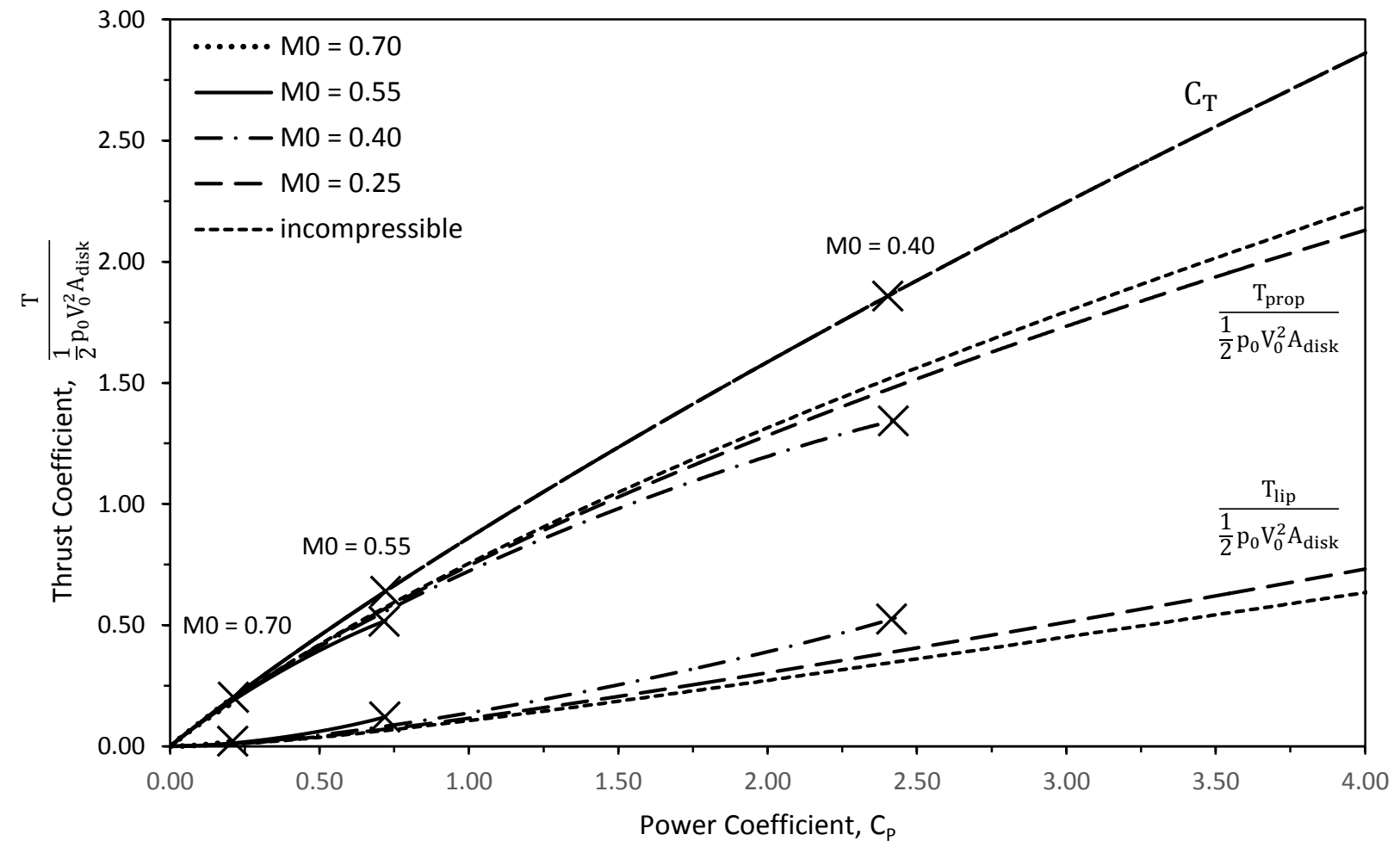

Figure 38. Contribution of the propeller thrust and lip thrust to the total thrust at various freestream Mach numbers for incompressible and compressible flow. 


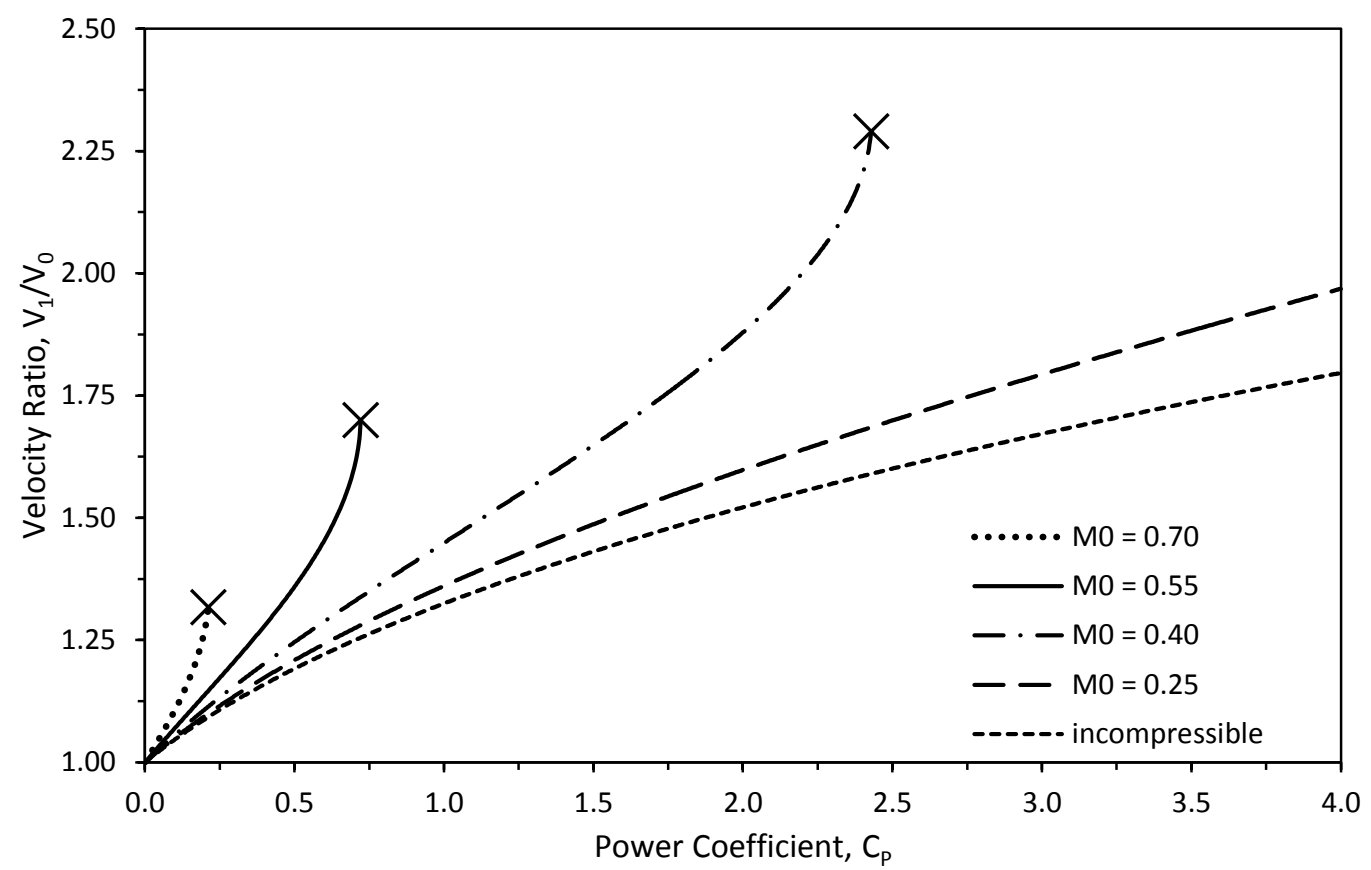

Figure 39. Comparison of the velocity immediately before the ducted propeller for incompressible flow and compressible flow of varying free-stream Mach numbers.

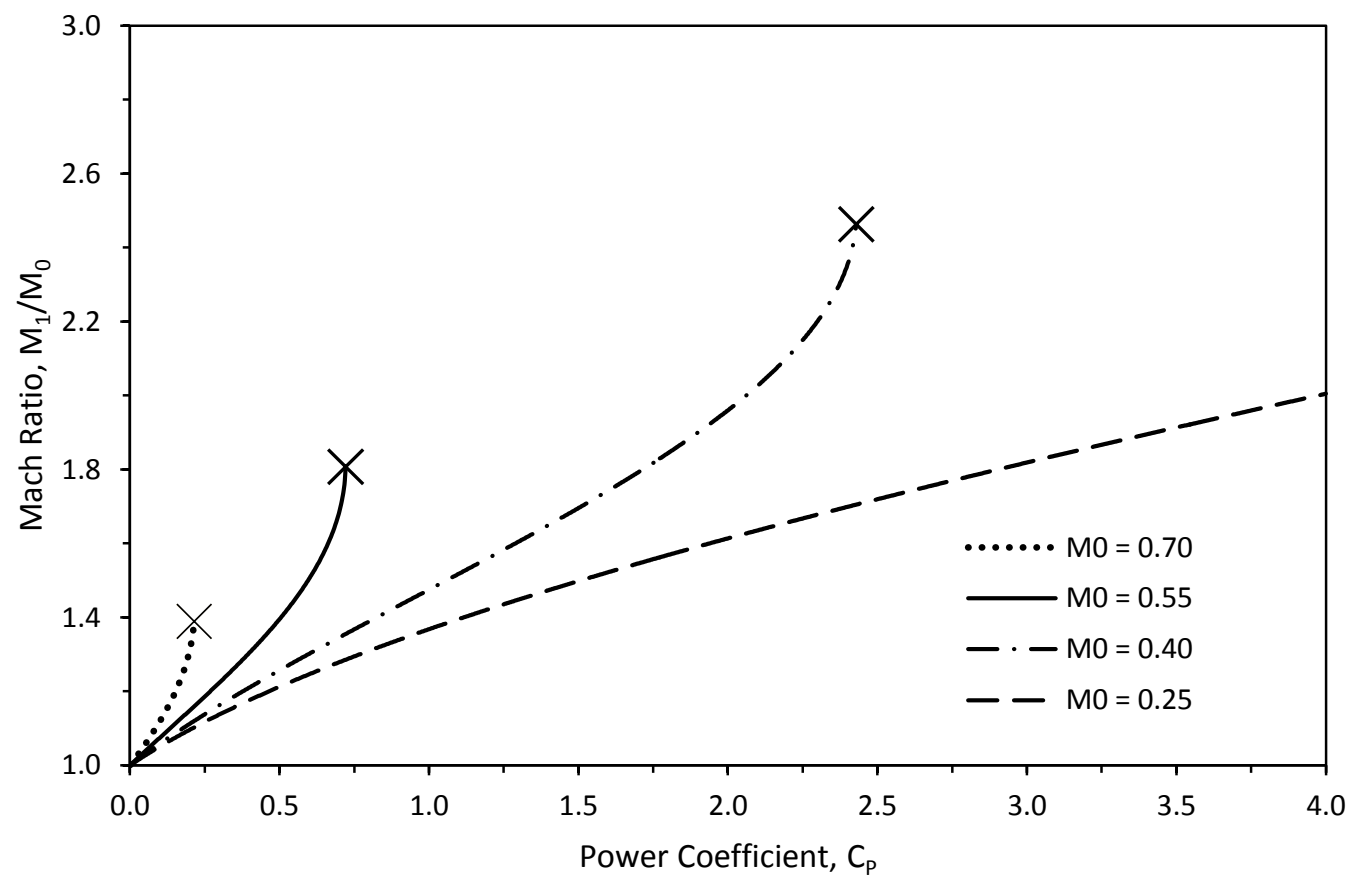

Figure 40. Comparison of the Mach number immediately before the ducted propeller for varying free-stream Mach numbers. 


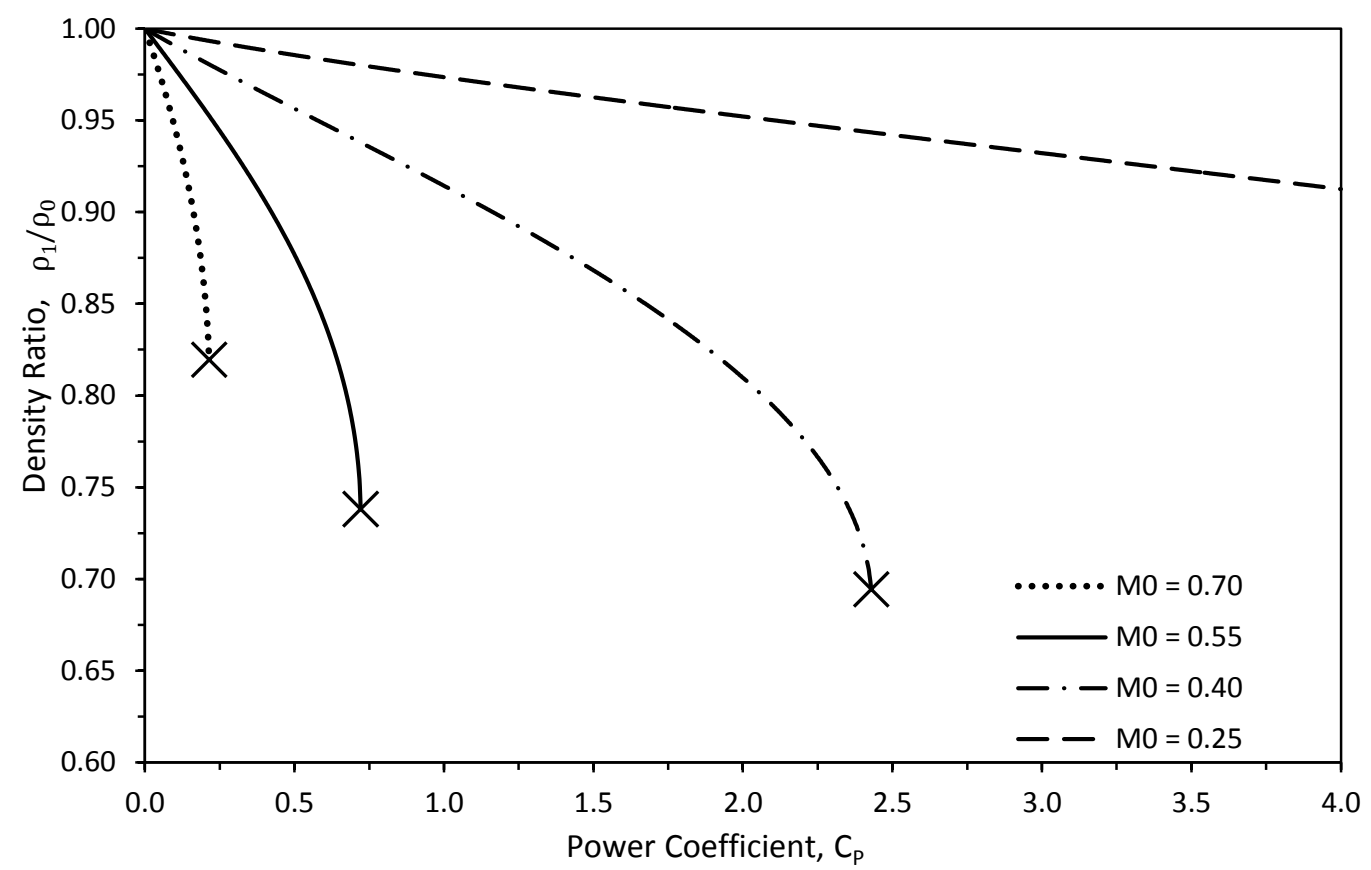

Figure 41. Comparison of the density immediately before the ducted propeller for varying freestream Mach numbers.

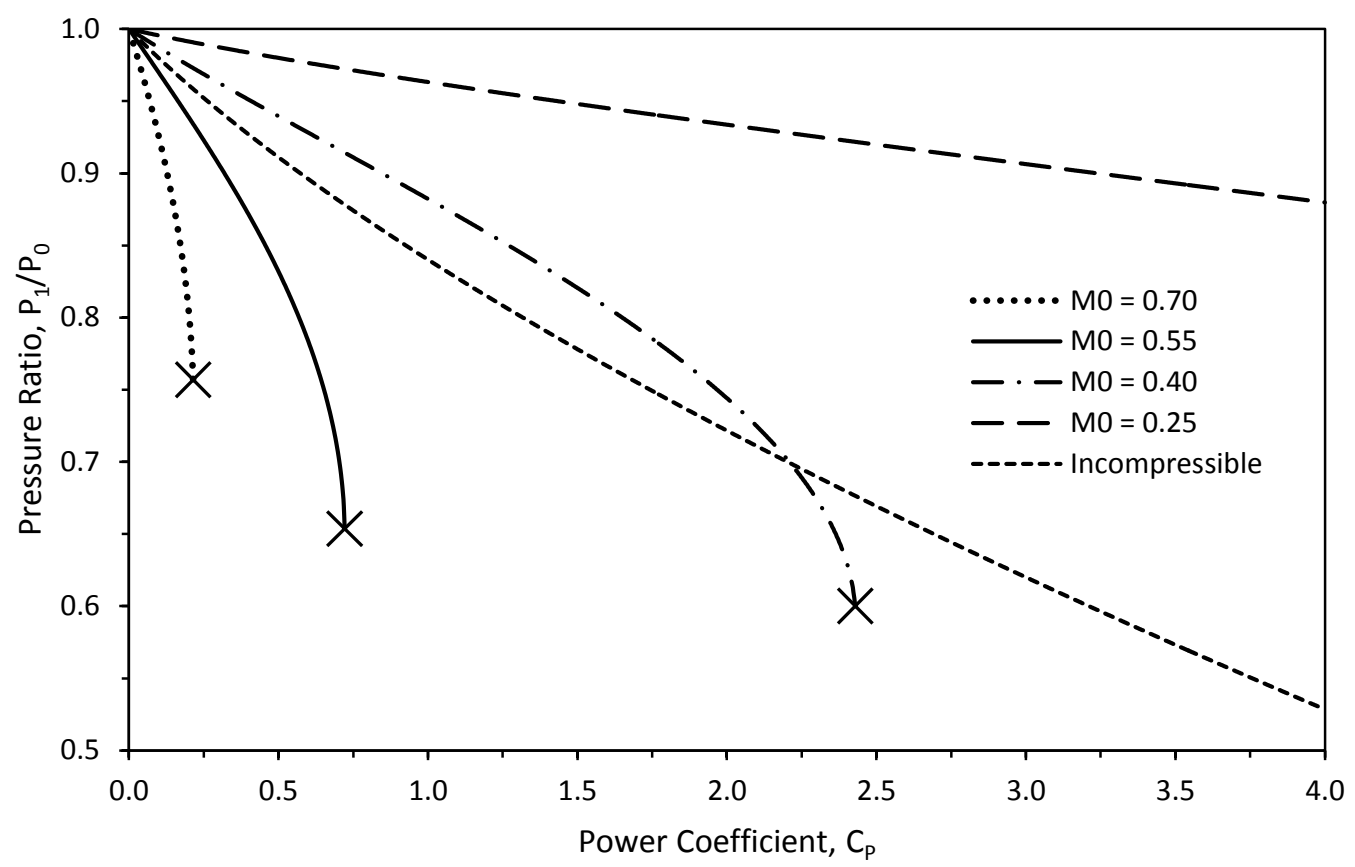

Figure 42. Comparison of the pressure immediately before the ducted propeller for incompressible flow and compressible flow of varying free-stream Mach numbers. 


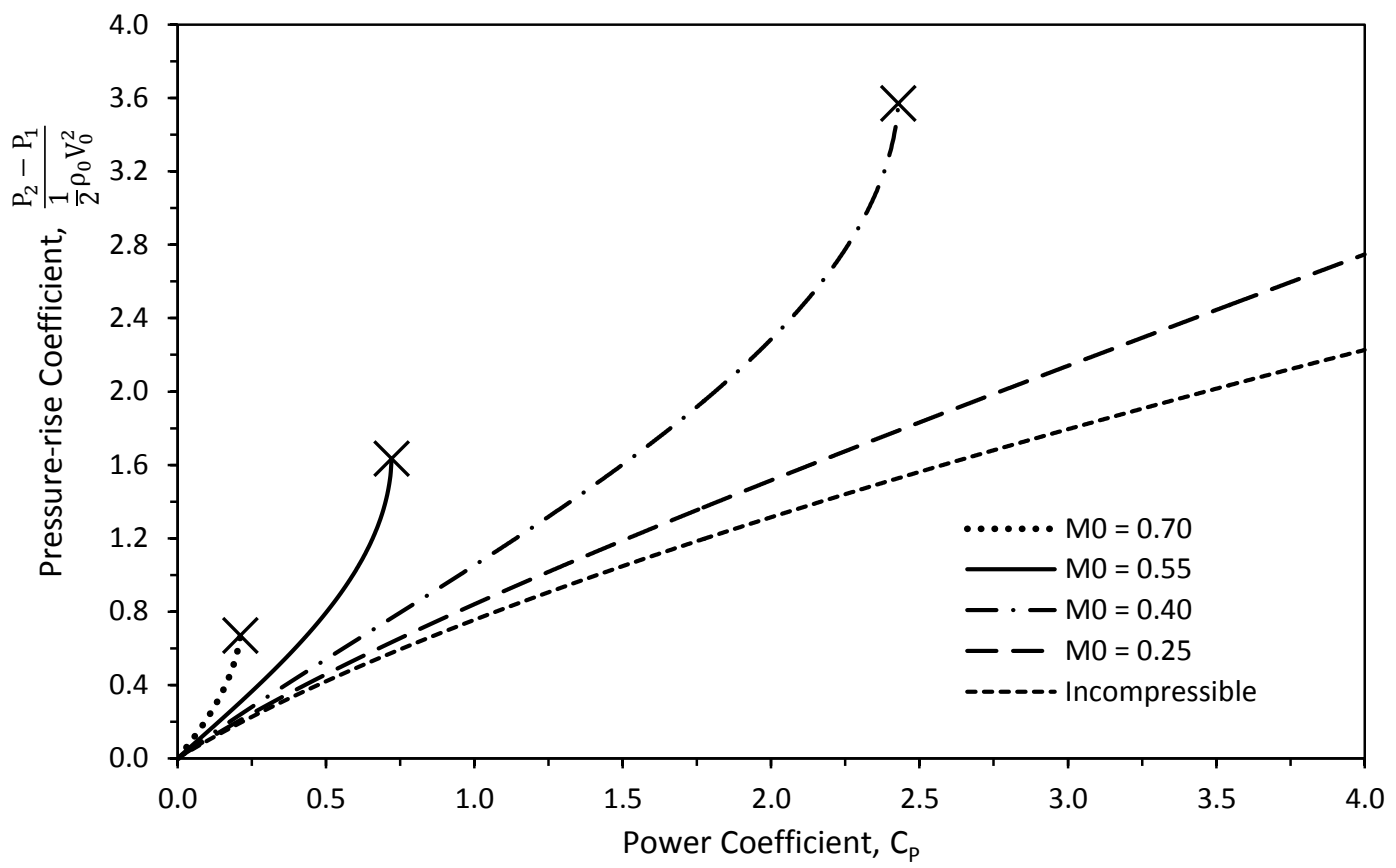

Figure 43. Comparison of the pressure rise across the disk for incompressible flow and compressible flow of varying free-stream Mach number for the ducted propeller.

\subsubsection{Discussion}

Figure 33 shows that the ducted propeller achieves the same propulsive efficiency for compressible and incompressible flow regardless of the free-stream Mach number. Figure 34 to Figure 37 also conveys that the dimensionless mass flowrate, the area ratio $\frac{A_{0}}{A_{\text {disk }}}$, the velocity ratio $\frac{V_{3}}{V_{0}}$, and the Mach ratio $\frac{M_{3}}{M_{0}}$ are the same for both incompressible and compressible flow, which all give consistent results. If $\frac{A_{0}}{A_{\text {disk }}}$ is the same for the ducted propeller regardless of the compressibility of the flow, then it must also be true for $\frac{\mathrm{V}_{3}}{\mathrm{~V}_{0}}$ and the dimensionless mass flowrate since they only depends on $\frac{A_{0}}{A_{\text {disk }}}$ other than the power coefficient. When compared to the bare propeller, the ducted propeller has a higher propulsive efficiency, which is also consistent with the results for the incompressible ducted propeller.

Figure 38 shows that the ducted propeller also achieves the same total thrust for both compressible and incompressible flow, but the individual contribution of the propeller thrust and 
the lip thrust to the total thrust differs not only between incompressible and compressible flow but also between varying free-stream Mach numbers for compressible flow.

Figure 39 to Figure 43 show that the velocity and thus the Mach number increase but the density and pressure are decrease immediately before the disk with increasing free-stream Mach numbers. When comparing between incompressible and compressible flow of the same freestream velocity, the drop across the disk is much greater for compressible flow than for incompressible flow as shown in Figure 43. 


\subsection{Chapter Summary}

In concluding remarks, it is found that the results of the bare propeller agree with those of Delano and Crigler [5]. When measured against the power coefficient, the propulsive efficiency of the bare propeller is approximately the same for both incompressible and compressible flow, regardless of the free-stream Mach number, up to the point where the flow immediately before the disk is sonic. When looking at the extreme cases of very low subsonic Mach numbers at very high power coefficients, the propulsive efficiency of the bare propeller does differ between

compressible and incompressible flow, but such cases are impractical in real applications of propellers. It is also found that the ducted propeller also achieves the same propulsive efficiency and has the same total thrust coefficient, regardless of compressibility or the free-stream Mach number. However, the individual contribution of the propeller thrust and the lip thrust to the total thrust differ greatly between compressible and incompressible flow. Agreements with the results of Delano and Crigler [5] not only builds confidence in the current method of solution and the compressible-flow results obtained for the bare and ducted propeller but also the results that will be presented for the compressible bare and ducted turbine, which is the main interest of the current study. The specific case where the free-stream velocity is zero for the bare and ducted compressible propeller is further investigated in Appendix C. 


\section{APPLICATIONS OF THE COMPRESSIBLE ACTUATOR DISK THEORY -}

\section{TURBINE}

\subsection{Introduction}

This chapter will focus on applying the compressible Actuator Disk Theory developed in Chapter 2 to the cases of the bare and ducted turbine. A turbine is an energy-extracting device that extracts useful energy from the total kinetic energy of the flow stream at the disk plane and experiences a drag force exerted by the flow. A well-known measure of its performance is its power extraction efficiency against the ratio of the far upstream and downstream velocities. Hence, the dimensionless parameters such as the power extraction efficiency and the total drag coefficient are established in this chapter for both the bare and ducted turbine.

The governing equations of the Compressible Actuator Disk Theory are used to determine the flow variables at each station and the performance of both the bare and ducted turbine in terms of the free-stream flow conditions, the disk area, and the velocity ratio at the far upstream to the far downstream. The solutions to the bare and ducted turbine are made dimensionless by the freestream conditions and are compared between varying free-stream Mach Numbers for compressible flow and between incompressible and compressible flow. It is important to note that this thesis is motivated by the need to derive the parameters that can be used to predict the performance of an ideal, small ram-air turbine (microRAT) [4] for high subsonic Mach number. Therefore, this chapter involving the compressible Actuator Disk Theory application for the bare and ducted turbine will be the main focus of the thesis.

To begin, the turbine is treated as an actuator disk having an area of $\mathrm{A}_{\text {disk }}$. Because the governing equations for the compressible Actuator Disk Theory are derived in Chapter 2 assuming that the disk is an energy-adding device, some of the equations will be tailored for an energy-extracting device, starting with the case of the bare turbine. 


\subsection{Compressible, Bare Turbine}

The power extraction efficiency and the total drag coefficient will be established for the compressible, bare turbine. Recall that the governing equations for the compressible Actuator Disk Theory are derived for an energy-adding device that exerts a thrust force on the flow. Since a turbine is a power-extracting device that experiences a drag force exerted by the flow, a change of sign is necessary to the power and the total thrust equations in Chapter 2. The power extracted by the turbine $\mathrm{P}_{\text {extracted }}$ is the negative of the input power $\mathrm{P}_{\text {input }}$ in equation 2-43 to signify that work is done on the turbine by the flow

$$
\mathrm{P}_{\text {extracted }}=-\mathrm{P}_{\text {input }}=\dot{\mathrm{m}} \frac{\mathrm{V}_{0}^{2}-\mathrm{V}_{3}^{2}}{2}
$$

The drag force on the turbine is in the opposite direction of the flow. So, the total drag $D_{\text {total }}$ exerted by the flow and the drag experienced by the turbine at the disk plane $\mathrm{D}_{\text {turb }}$ are in the opposite direction of the total thrust $\mathrm{T}_{\text {total }}$ and the disk thrust $\mathrm{T}_{\text {disk }}$ given in equation 2-41 and 242

$$
\begin{gathered}
\mathrm{D}_{\text {total }}=-\mathrm{T}_{\text {total }}=\dot{\mathrm{m}}\left(\mathrm{V}_{0}-\mathrm{V}_{3}\right) \\
\mathrm{D}_{\text {turb }}=-\mathrm{T}_{\text {disk }}=\dot{\mathrm{m}}\left(\mathrm{V}_{1}-\mathrm{V}_{2}\right)+\mathrm{A}_{\text {disk }}\left(\mathrm{P}_{1}-\mathrm{P}_{2}\right)
\end{gathered}
$$

Since the turbine is the only assembly within the stream tube, the total drag exerted by the flow is equal to the drag experienced by the turbine, analogous to the equivalence of the total thrust and the disk thrust for the compressible actuator disk

$$
\mathrm{D}_{\text {total }}=\mathrm{D}_{\text {turb }}
$$

Next the power extraction efficiency and the total drag coefficient are established. The turbine's power extraction efficiency is introduced by dividing the extracted power in equation 4-2 by the available power in the free-stream in an area equal to $A_{\text {disk }}$

$$
\eta_{\text {turb }}=\frac{P_{\text {extracted }}}{P_{\text {available }}}=\frac{\dot{m} \frac{v_{0}^{2}-v_{3}^{2}}{2}}{\frac{1}{2} \rho_{0} A_{\text {disk }} V_{0}^{3}}
$$


Similarly, the total drag coefficient is found by dividing the total drag in equation 4-1 by the reference force

$$
C_{D}=\frac{D_{\text {total }}}{\frac{1}{2} \rho_{0} A_{\text {disk }} V_{0}^{2}}=\frac{\dot{\mathrm{m}}\left(V_{0}-V_{3}\right)}{\frac{1}{2} \rho_{0} A_{\text {disk }} V_{0}^{2}}
$$

An alternative form of the power extraction efficiency and the total drag coefficient will be shown. By substituting the following mass flowrate into equation 4-6 and simplifying,

$$
\dot{\mathrm{m}}=\rho_{0} \mathrm{~A}_{0} \mathrm{~V}_{0}
$$

the turbine extraction efficiency becomes

$$
\eta_{\text {turb }}=\frac{A_{0}}{A_{\text {disk }}}\left(1-\left(\frac{V_{3}}{V_{0}}\right)^{2}\right)
$$

Likewise, the total drag coefficient can be simplified to the following form using the mass flowrate in equation $4-7$

$$
C_{D}=2 \frac{A_{0}}{A_{\text {disk }}}\left(1-\frac{V_{3}}{V_{0}}\right)
$$

While this form of the turbine extraction efficiency and the total drag coefficient may seem unconventional, it is useful when comparing the results that are presented later in the chapter. With the performance parameters established, the complete set of the governing equations for the compressible bare turbine are summarized in the next section. 
4.2.1. Summary of Governing Equations

$$
\begin{aligned}
& M_{0}=V_{0}\left(\gamma \cdot \frac{P_{0}}{\rho_{0}}\right)^{-\frac{1}{2}} \\
& \frac{A_{0}}{A_{\text {disk }}}=\frac{M_{1}}{M_{0}}\left(\frac{1+\frac{\gamma-1}{2} M_{0}^{2}}{1+\frac{\gamma-1}{2} M_{1}^{2}}\right)^{\frac{\gamma+1}{2(\gamma-1)}} \\
& \frac{\mathrm{P}_{1}}{\mathrm{P}_{0}}=\left(\frac{1+\frac{\gamma-1}{2} \mathrm{M}_{0}^{2}}{1+\frac{\gamma-1}{2} \mathrm{M}_{1}^{2}}\right)^{\frac{\gamma}{\gamma-1}} \\
& \frac{\rho_{1}}{\rho_{0}}=\left(\frac{\mathrm{P}_{1}}{\mathrm{P}_{0}}\right)^{\frac{1}{\gamma}} \\
& \frac{\mathrm{V}_{1}}{\mathrm{~V}_{0}}=\frac{\rho_{0}}{\rho_{1}} \frac{\mathrm{A}_{0}}{\mathrm{~A}_{\mathrm{disk}}} \\
& M_{3}=V_{3}\left(\gamma \cdot \frac{P_{0}}{\rho_{0}}\right)^{-\frac{1}{2}} \\
& \frac{A_{3}}{A_{0}}=\frac{V_{0}}{V_{3}} \\
& \frac{A_{3}}{A_{\text {disk }}}=\frac{M_{2}}{M_{3}}\left(\frac{1+\frac{\gamma-1}{2} M_{3}^{2}}{1+\frac{\gamma-1}{2} M_{2}^{2}}\right)^{\frac{\gamma+1}{2(\gamma-1)}} \\
& \frac{P_{2}}{P_{0}}=\left(\frac{1+\frac{\gamma-1}{2} M_{3}^{2}}{1+\frac{\gamma-1}{2} M_{2}^{2}}\right)^{\frac{\gamma}{\gamma-1}} \\
& \frac{\rho_{2}}{\rho_{0}}=\left(\frac{\mathrm{P}_{2}}{\mathrm{P}_{0}}\right)^{\frac{1}{\gamma}} \\
& \mathrm{V}_{2}=\frac{\rho_{1}}{\rho_{2}} \mathrm{~V}_{1} \\
& \dot{\mathrm{m}}=\rho_{1} \mathrm{~A}_{\mathrm{disk}} \mathrm{V}_{1} \\
& \mathrm{P}_{\text {extracted }}=\dot{\mathrm{m}} \frac{\mathrm{V}_{0}^{2}-\mathrm{V}_{3}^{2}}{2} \\
& \mathrm{D}_{\text {total }}=\dot{\mathrm{m}}\left(\mathrm{V}_{0}-\mathrm{V}_{3}\right) \\
& \mathrm{D}_{\text {turb }}=\dot{\mathrm{m}}\left(\mathrm{V}_{1}-\mathrm{V}_{2}\right)+\mathrm{A}_{\text {disk }}\left(\mathrm{P}_{1}-\mathrm{P}_{2}\right) \\
& \mathrm{D}_{\text {total }}=\mathrm{D}_{\text {turb }} \\
& C_{D}=2 \frac{A_{0}}{A_{\text {disk }}}\left(1-\frac{V_{3}}{V_{0}}\right)
\end{aligned}
$$




$$
\eta_{\text {turb }}=\frac{A_{0}}{A_{\text {disk }}}\left(1-\left(\frac{V_{3}}{V_{0}}\right)^{2}\right)
$$

These governing equations provide the necessary system of equations to determine the flow variables throughout the slipstream and the performance of the turbine in terms of the free-stream conditions, the disk area, and the far downstream velocity.

\subsubsection{Method of Solution}

The governing equations are derived for given free-stream conditions which are $\gamma, \rho_{0}, P_{0}$, and $V_{0}$, the disk area $A_{\text {disk }}$, and the far downstream velocity $V_{3}$. With these six inputs, the unknown variables left to determine are the following flow variables $A_{0}, M_{0}, M_{1}, \rho_{1}, P_{1}, V_{1}, M_{2}, \rho_{2}, P_{2}, V_{2}$, $A_{3}, M_{3}$ and the turbine's performance parameters $D_{\text {turb }}, D_{\text {total }}, P_{\text {extracted }}, C_{D}, \eta_{\text {turb }}$, and $\dot{m}$, all of which sums to eighteen unknown variables. These eighteen unknown variables can be solved using the eighteen non-linear coupled equations summarized in section 4.2.1.

The main variable of interest, the extracted power $\mathrm{P}_{\text {extracted }}$, is nondimensionalized using Buckingham Pi Theorem to find the dimensionless independent variables. $\mathrm{P}_{\text {extracted }}$ can be expressed as a function of the given variables $\gamma, V_{0}, \rho_{0}, P_{0}, A_{\text {disk }}$, and $V_{3}$. With a total of seven variables and three physical dimensions, the four resulting nondimensional parameters are $\frac{\mathrm{P}_{\text {extracted }}}{\frac{1}{2} \rho_{0} \mathrm{~A}_{\text {disk }} \mathrm{V}_{0}^{3}}, \mathrm{~V}_{0}\left(\gamma \cdot \frac{\mathrm{P}_{0}}{\rho_{0}}\right)^{-\frac{1}{2}}, \quad \gamma$, and $\frac{\mathrm{V}_{3}}{\mathrm{~V}_{0}}$ of which the first and second are the power extraction

efficiency and the free-stream Mach number $M_{0}$. The velocity ratio $\frac{V_{3}}{V_{0}}$, which will be denoted by $\mathrm{r}$, and the free-stream Mach number $\mathrm{M}_{0}$ are made into independent variables while $\gamma$ is kept as a constant. Note that $r$ can never be zero since it is impossible to extract $100 \%$ of the flow's kinetic energy into useful power. The maximum value of $r$ is 1 which corresponds to no power extraction made by the turbine. Therefore, for a given $\gamma, \rho_{0}, P_{0}$, and $A_{\text {disk }}$, the eighteen unknown variables are solved in Engineering Equation Solver (EES) in terms of r, ranging from 0.001 to 1 , for varying values of $\mathrm{M}_{0}$. 
Lastly, the solutions are made dimensionless by the free-stream flow conditions and the disk area to give the following nondimensional parameters $\frac{\rho_{1}}{\rho_{0}}, \frac{\rho_{2}}{\rho_{0}}, \frac{P_{1}}{P_{0}}, \frac{P_{2}}{P_{0}}, \frac{V_{1}}{V_{0}}, \frac{V_{2}}{V_{0}}, \frac{M_{1}}{M_{0}}, \frac{M_{2}}{M_{0}}, \frac{A_{0}}{A_{\text {disk }}}, \frac{\dot{m}}{\rho_{0} V_{0} A_{\text {disk }}}$ and $\frac{A_{3}}{A_{\text {disk }}}$. The pressure-difference across the disk is also non-dimensionalized by the dynamic pressure associated with the free-stream velocity introducing the pressure-difference coefficient $\frac{\mathrm{P}_{2}-\mathrm{P}_{1}}{\frac{1}{2} \rho_{0} \mathrm{~V}_{0}^{2}}$, which is positive for a pressure rise and negative for a pressure drop across the disk. Note that due to the implicit nature of the compressible flow equations given in section 4.2.1, it is more effective to turn the dimensional solutions obtained in EES into dimensionless parameters than it is to develop dimensionless representations of the flow variables. As a result, dimensional equations are presented but dimensionless solutions are presented in the next section.

\subsubsection{Results}

The compressible-flow solutions are determined for the disk area and the sea level static freestream flow conditions given in Table 1 and for the free-stream Mach numbers of 0.4, 0.6, and 0.8 , as was done for the compressible propeller. To see the effects of compressibility on the flow variables, incompressible-flow solutions are also obtained for the same given variables in Table 1 and for the free-stream velocity $V_{0}$ equivalent to Mach 0.6 at sea level static conditions.

The Betz limit for the compressible bare turbine and the velocity ratio $r$ at which it occurs are given in Table 3 for $\mathrm{M}_{0}$ ranging from 0.4 to 0.8 . The effect of the free-stream Mach number on the Betz limit and its corresponding velocity ratio $r$ is also presented in Figure 45 to Figure 47. The power extraction efficiency, the total drag coefficient, the dimensionless mass flowrate, the ratios of the far upstream and downstream areas to the disk area, and the dimensionless flow variables immediately before and after the disk are plotted against the velocity ratio $r$ for $\mathrm{M}_{0}$ of 0.4, 0.6, and 0.8 in Figure 44 and Figure 48 to Figure 57. When applicable, the dimensionless 
incompressible-flow solutions for $\mathrm{V}_{0}$ equivalent to Mach 0.6 at sea level static conditions are also included in the plots. These plots are discussed in the next section.

Table 3. Table of the Betz limit for the bare turbine between compressible and incompressible flow.

\begin{tabular}{|c|c|c|c|c|c|c|}
\hline $\mathrm{M}_{0}$ & 0.8 & 0.7 & 0.6 & 0.5 & 0.4 & Incompressible \\
\hline velocity ratio & 0.297 & 0.307 & 0.315 & 0.321 & 0.325 & 0.333 \\
\hline Betz limit & 0.614 & 0.609 & 0.605 & 0.601 & 0.598 & 0.593 \\
\hline
\end{tabular}

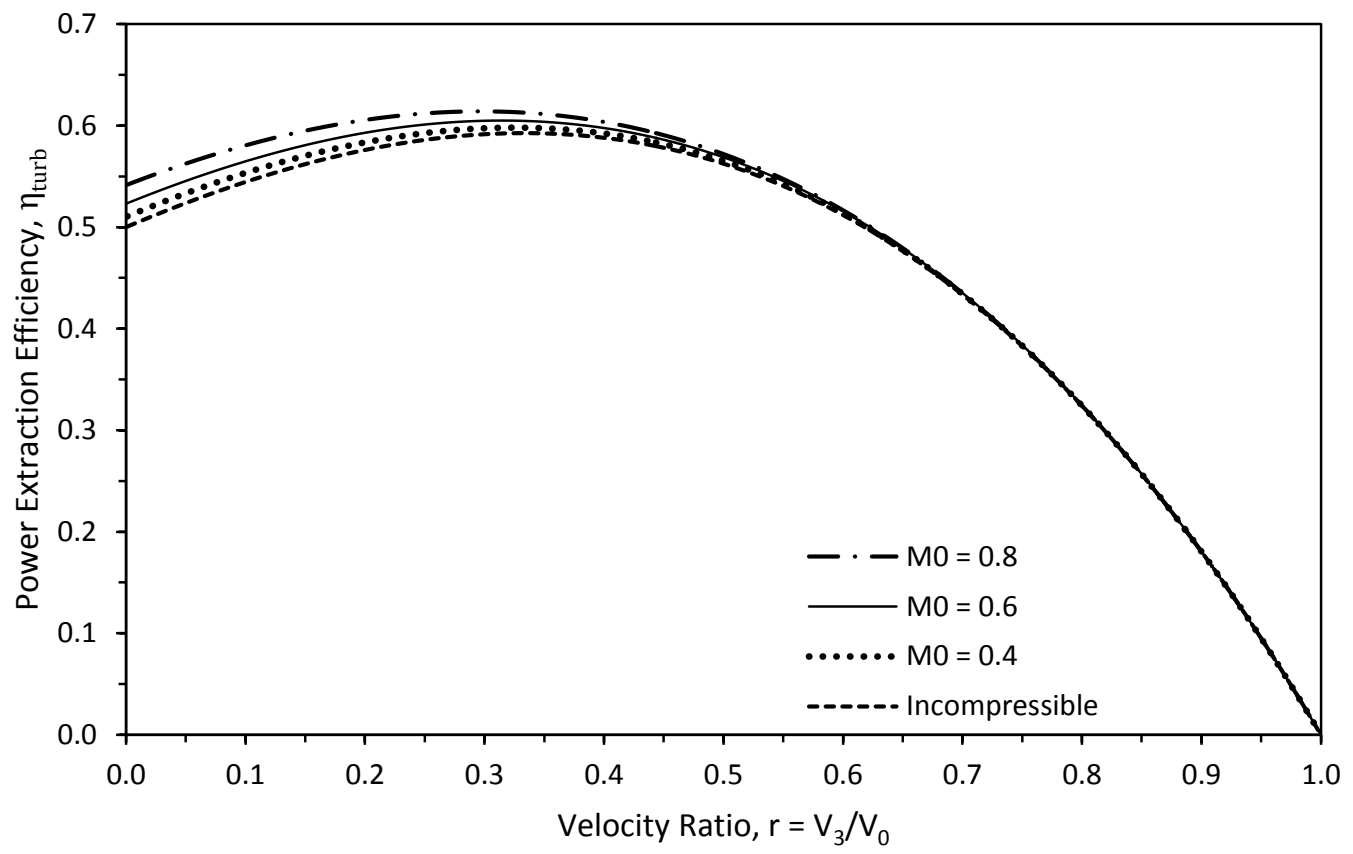

Figure 44. Comparison of the bare turbine's power extraction efficiency for incompressible flow and for compressible flow of varying free-stream Mach numbers. 


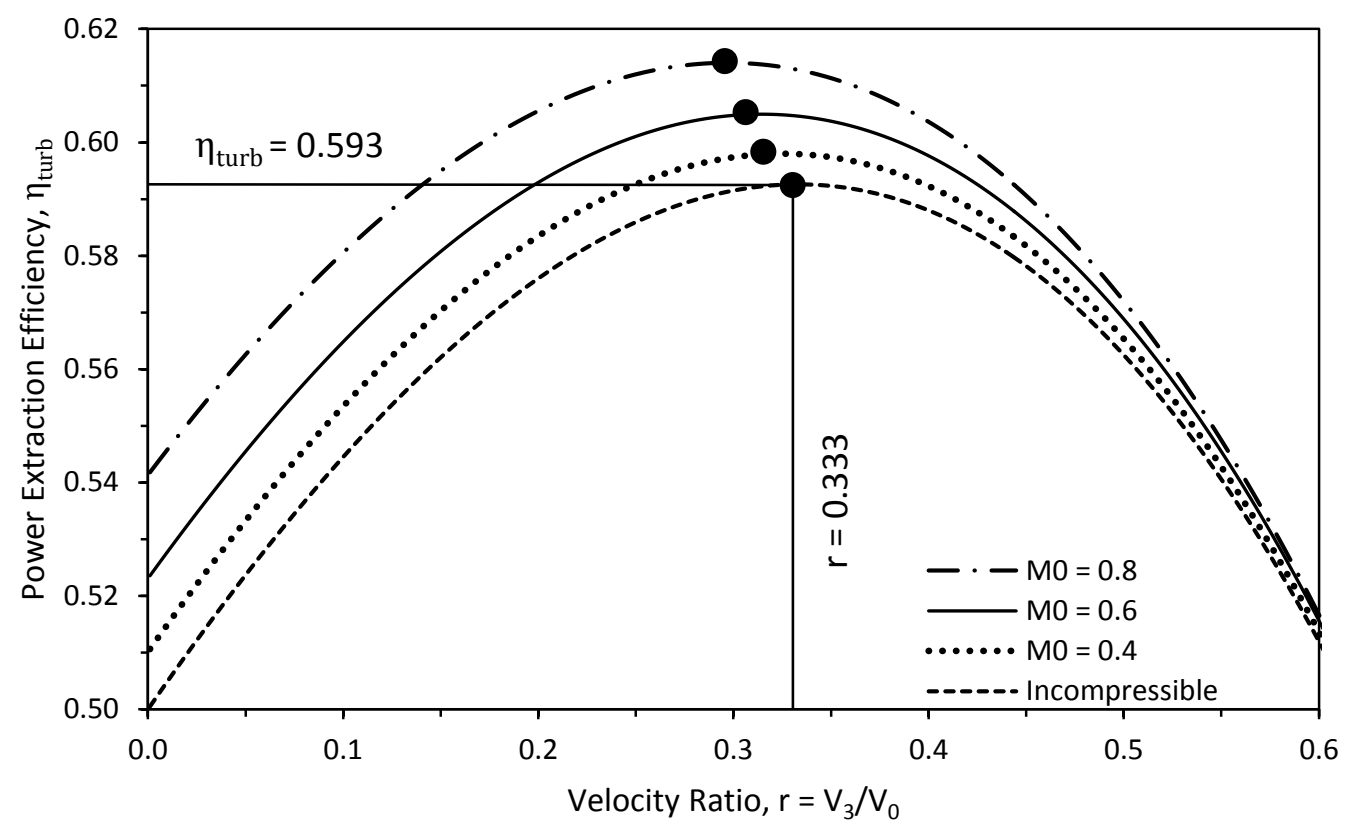

Figure 45. Comparison of the Betz Limit of the bare turbine for incompressible flow and for compressible flow of varying free-stream Mach numbers. The black dots represent the Betz Limit of each curve: note that as $\mathbf{M}_{\mathbf{0}}$ increases, the black dot slightly moves higher and more to the left.

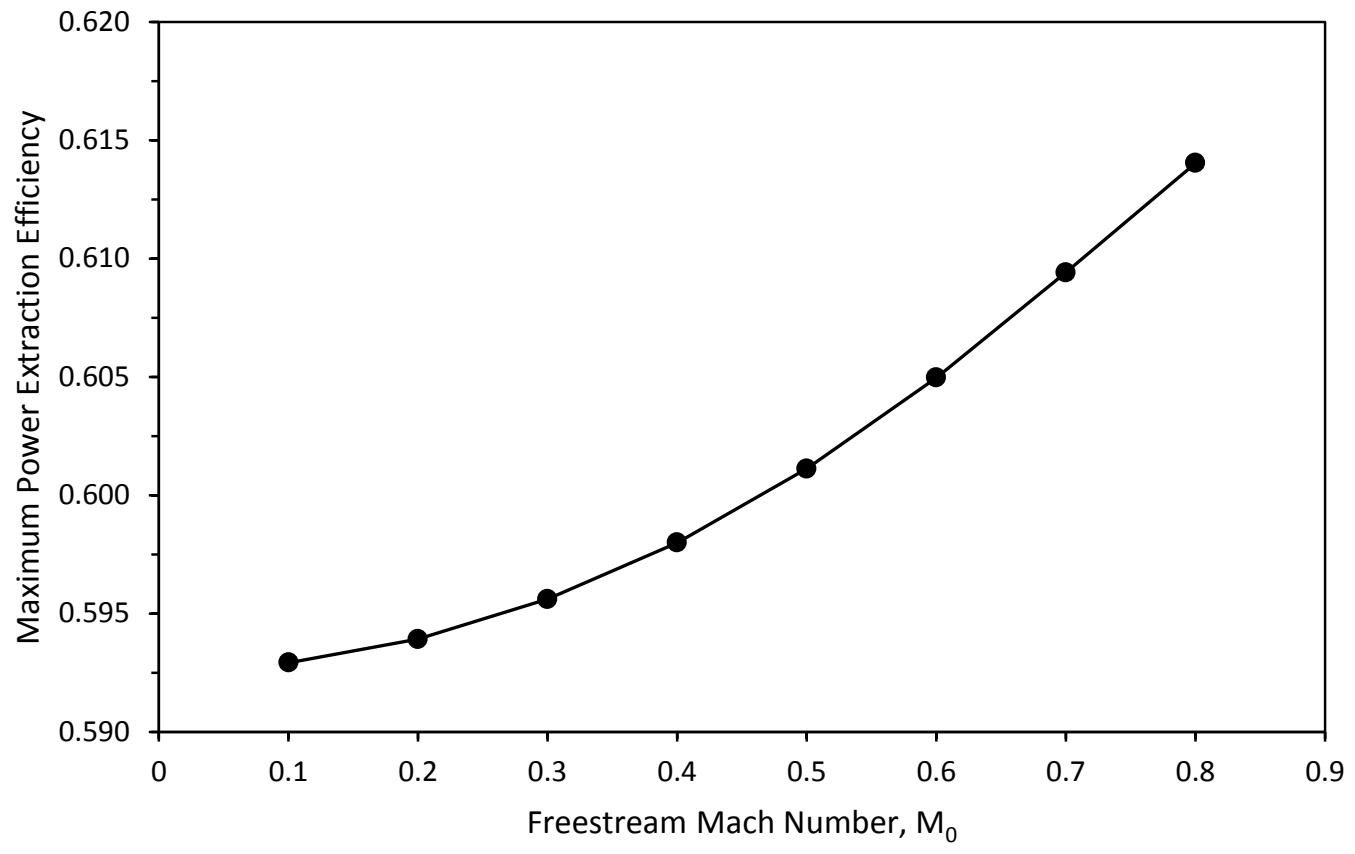

Figure 46. The Betz Limit (maximum power extraction efficiency) versus the free-stream Mach number $\mathbf{M}_{\mathbf{0}}$ for the compressible, bare turbine. 


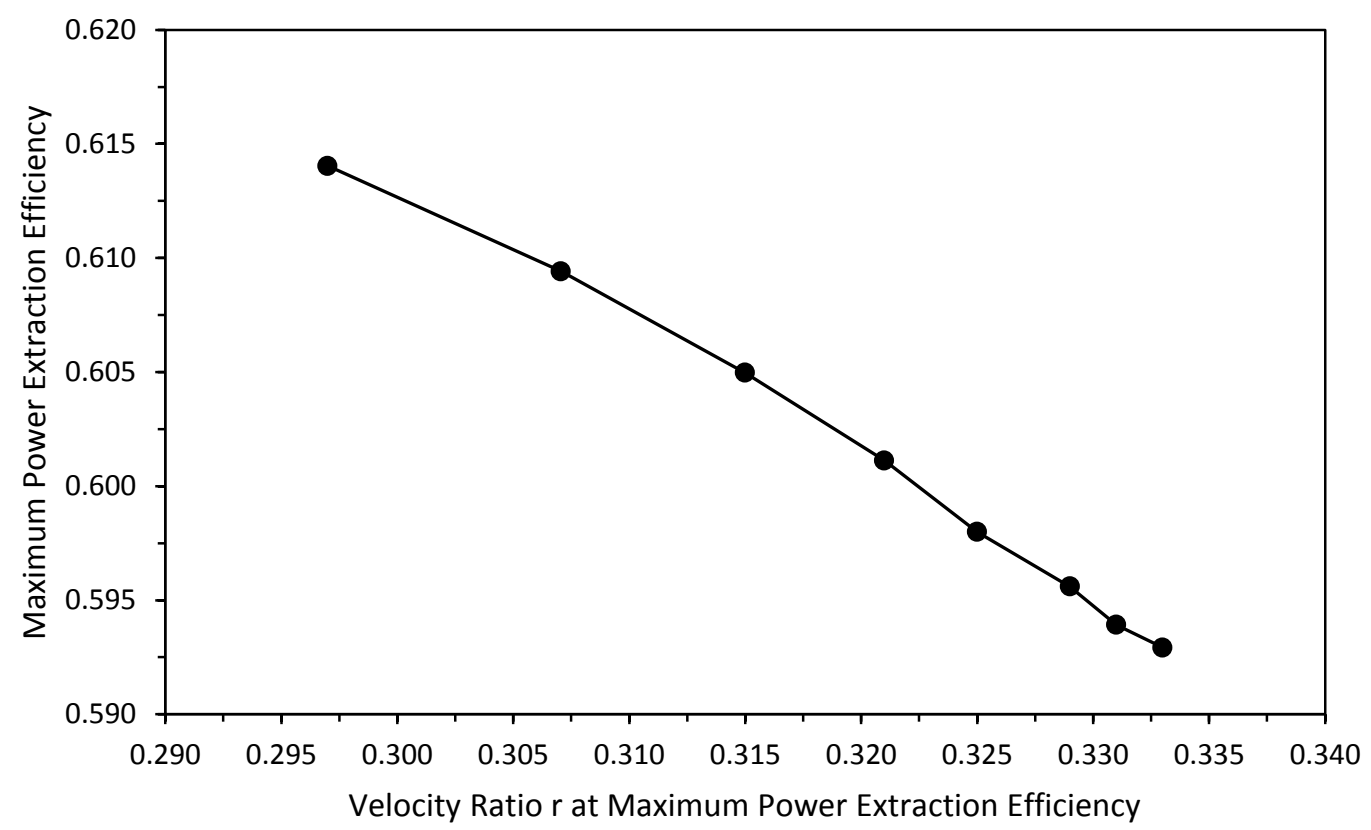

Figure 47. The Betz Limit (maximum power extraction efficiency) versus the corresponding velocity ratio $r$ for the compressible, bare turbine.

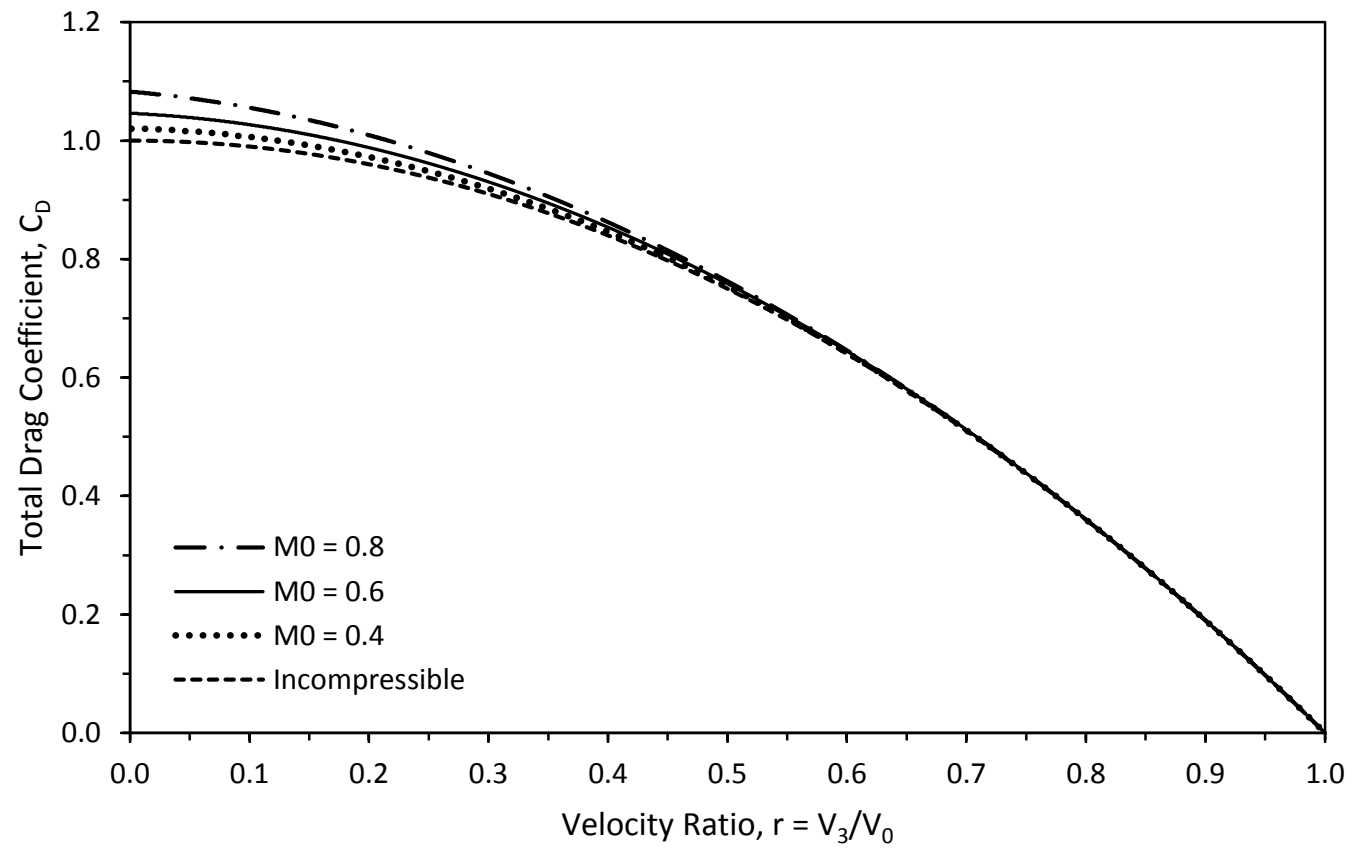

Figure 48. Comparison of the bare turbine's total drag coefficient for incompressible flow and for compressible flow of varying free-stream Mach numbers. 


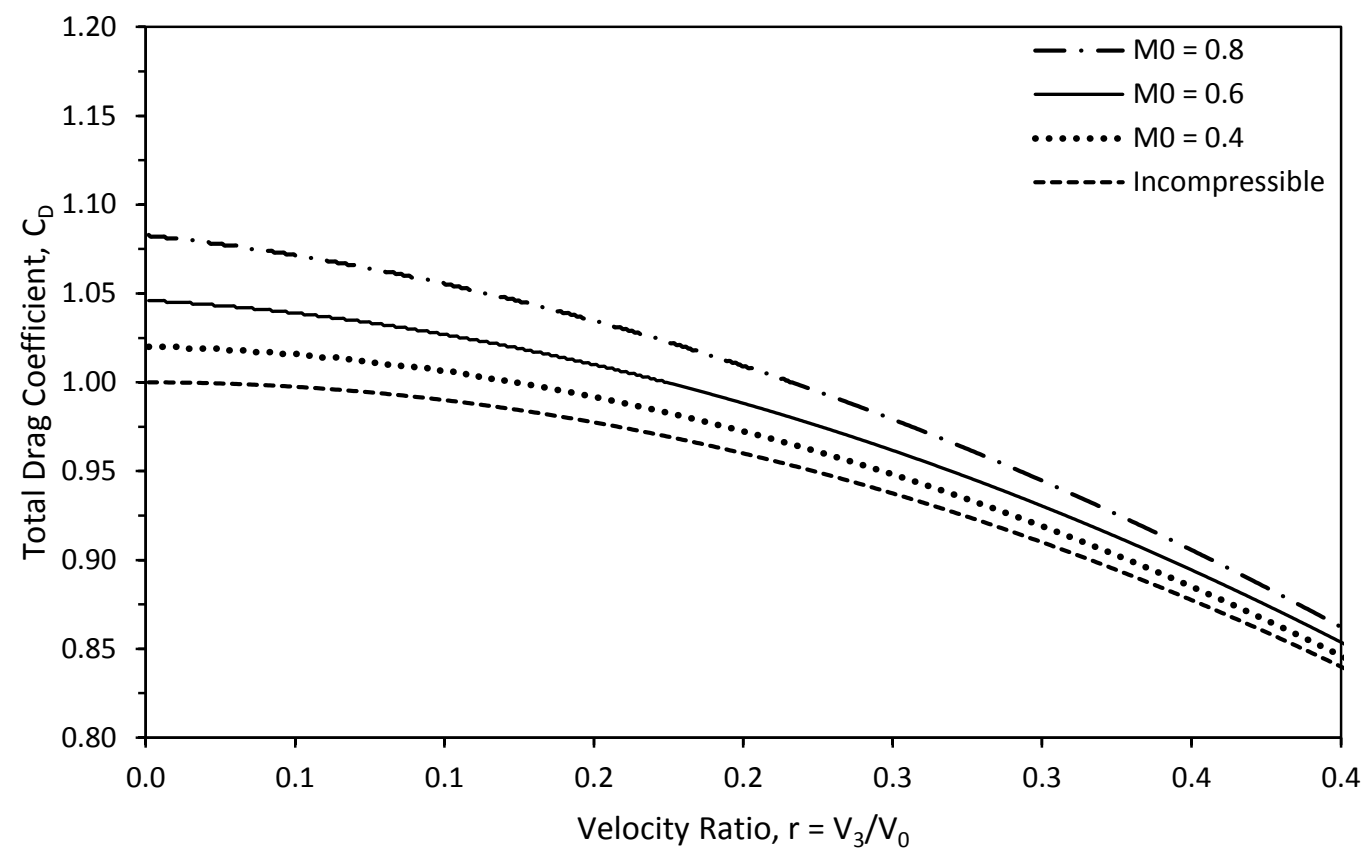

Figure 49. Comparison of the bare turbine's total drag coefficient for incompressible flow and for compressible of varying free-stream Mach numbers within a smaller domain and range (enlarged).

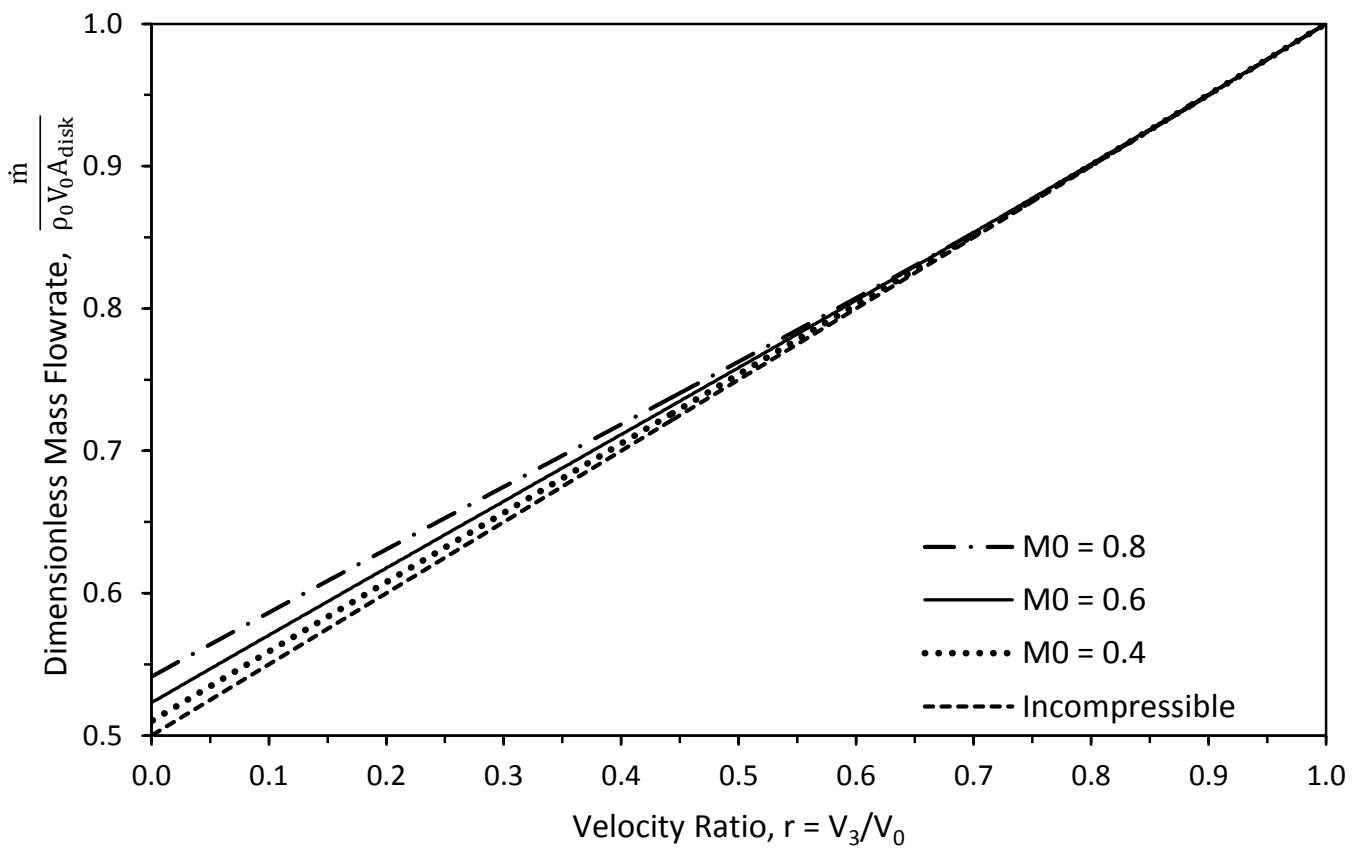

Figure 50. Comparison of the bare turbine's dimensionless mass flowrate for incompressible flow and compressible flow of varying free-stream Mach numbers. 


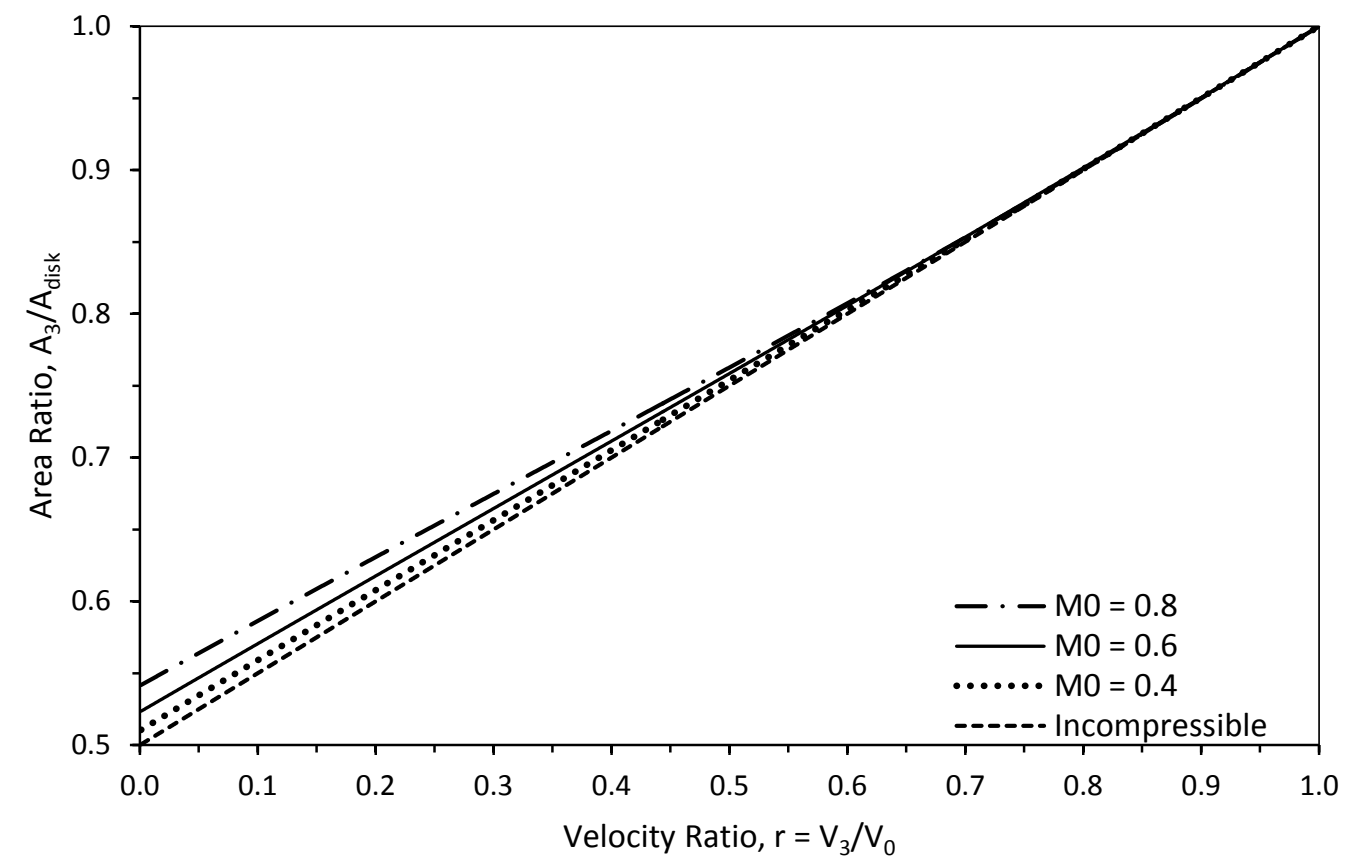

Figure 51. Comparison of the area at the far downstream between incompressible flow and compressible flow of varying free-stream Mach numbers for the bare turbine.

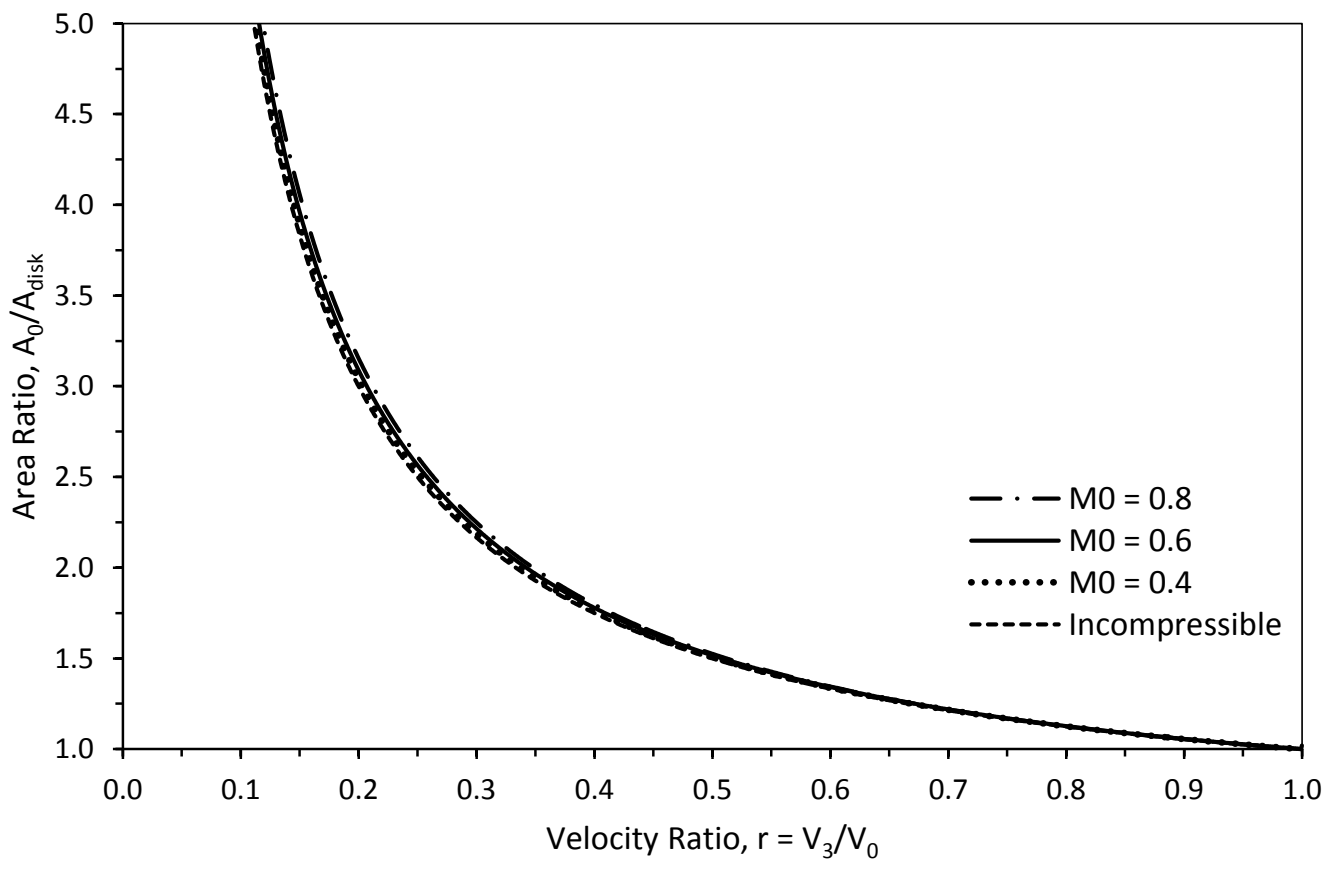

Figure 52. Comparison of the area at the far upstream between incompressible flow and compressible flow of varying free-stream Mach numbers for the bare turbine. Note that $\frac{\mathbf{A}_{\mathbf{0}}}{\mathbf{A}_{\mathbf{d i s k}}}$ approaches infinity as $r$ goes to zero. 


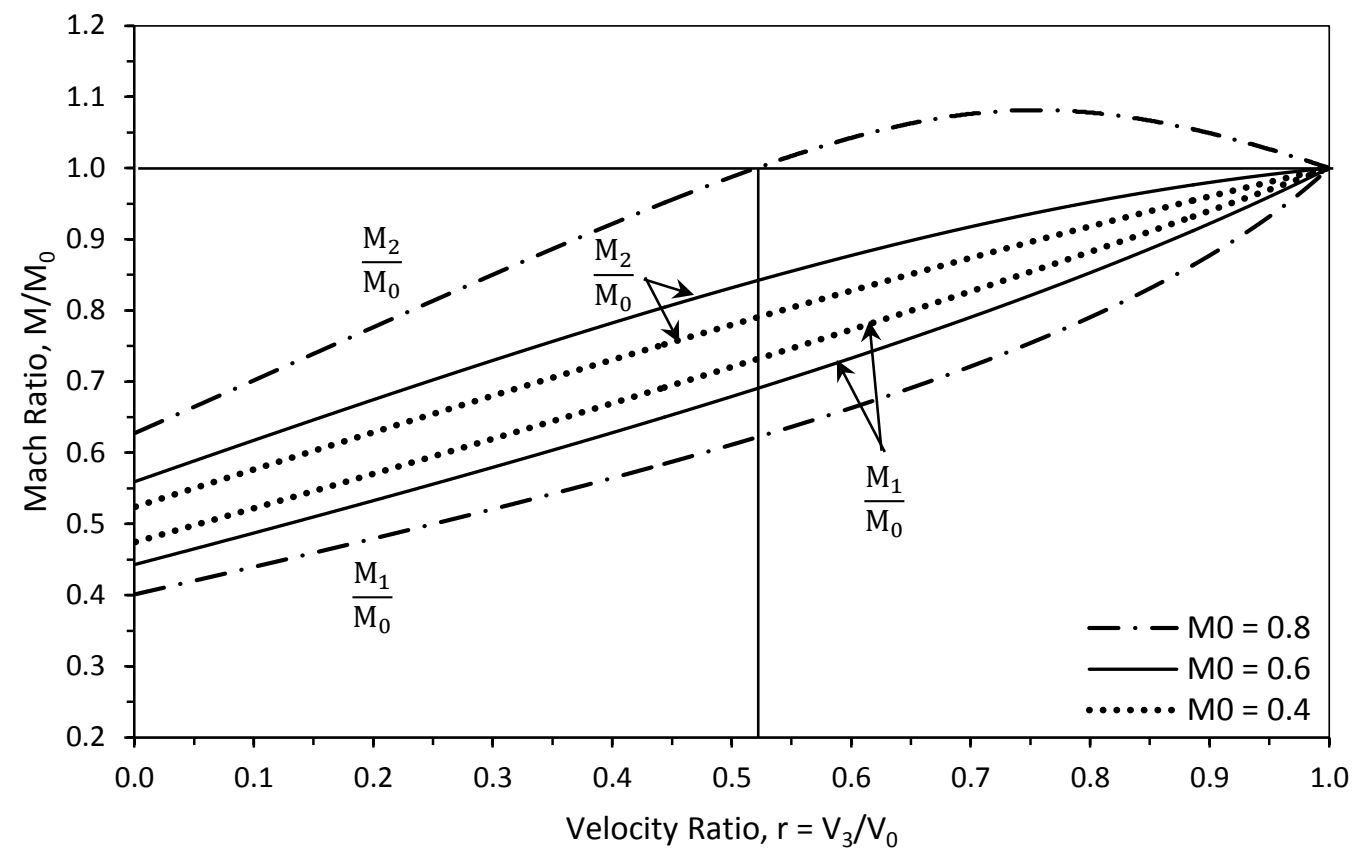

Figure 53. Comparison of the Mach numbers immediately before and after the bare turbine for varying free-stream Mach numbers. Note that $\mathbf{M}_{2}$ surpasses $\mathbf{M}_{\mathbf{0}}$ between an $\mathrm{r}$ value of 0.5 and 0.55 for the curve $\mathbf{M}_{\mathbf{0}}=\mathbf{0 . 8}$.

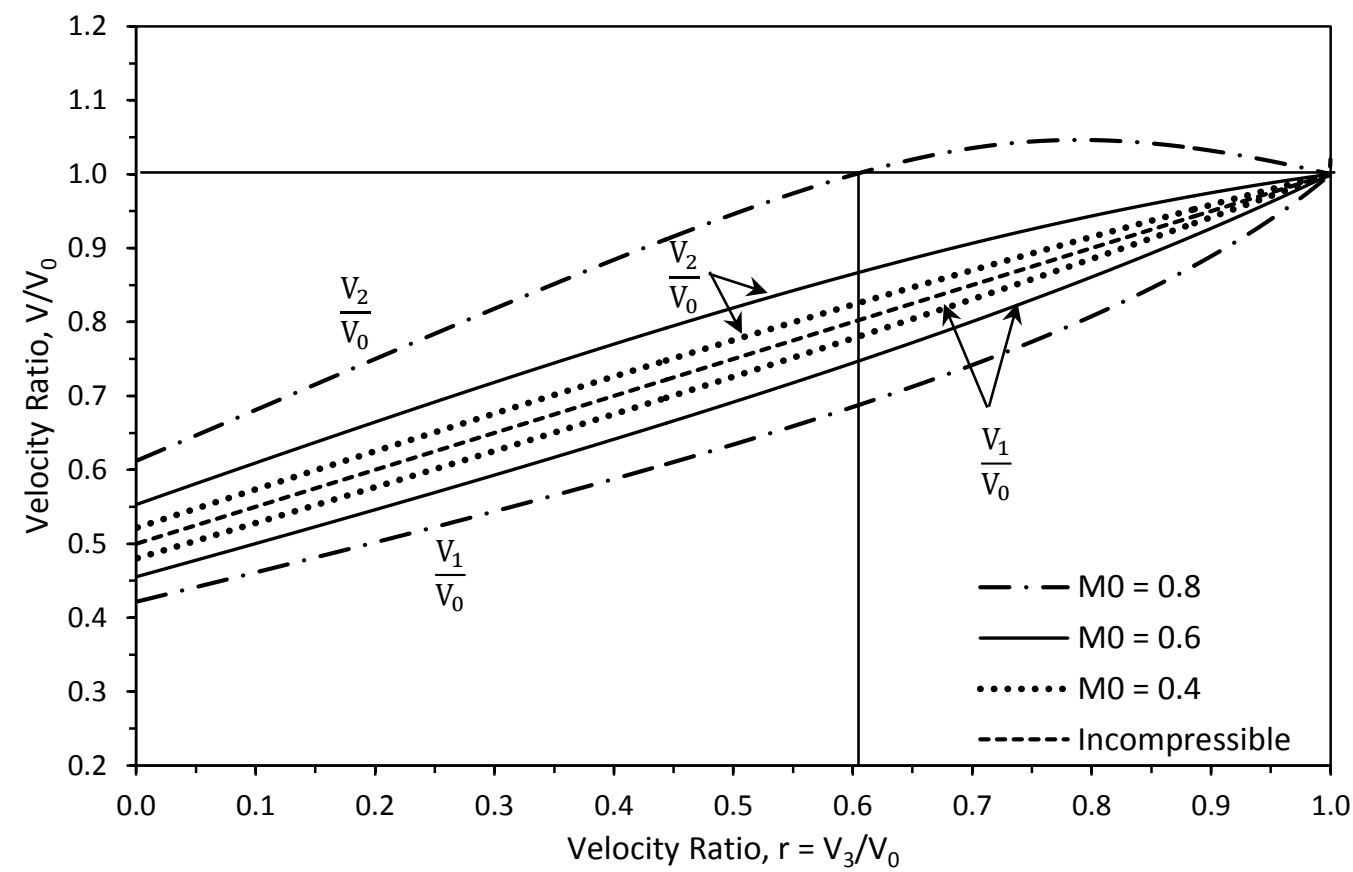

Figure 54. Comparison of velocities immediately before and after the bare turbine for incompressible flow and compressible flow of varying free-stream Mach numbers. Note that $\mathbf{V}_{\mathbf{2}}$ surpasses $\mathbf{V}_{\mathbf{0}}$ between an $\mathrm{r}$ value of 0.6 and 0.65 for the curve $\mathbf{M}_{\mathbf{0}}=\mathbf{0 . 8}$. 


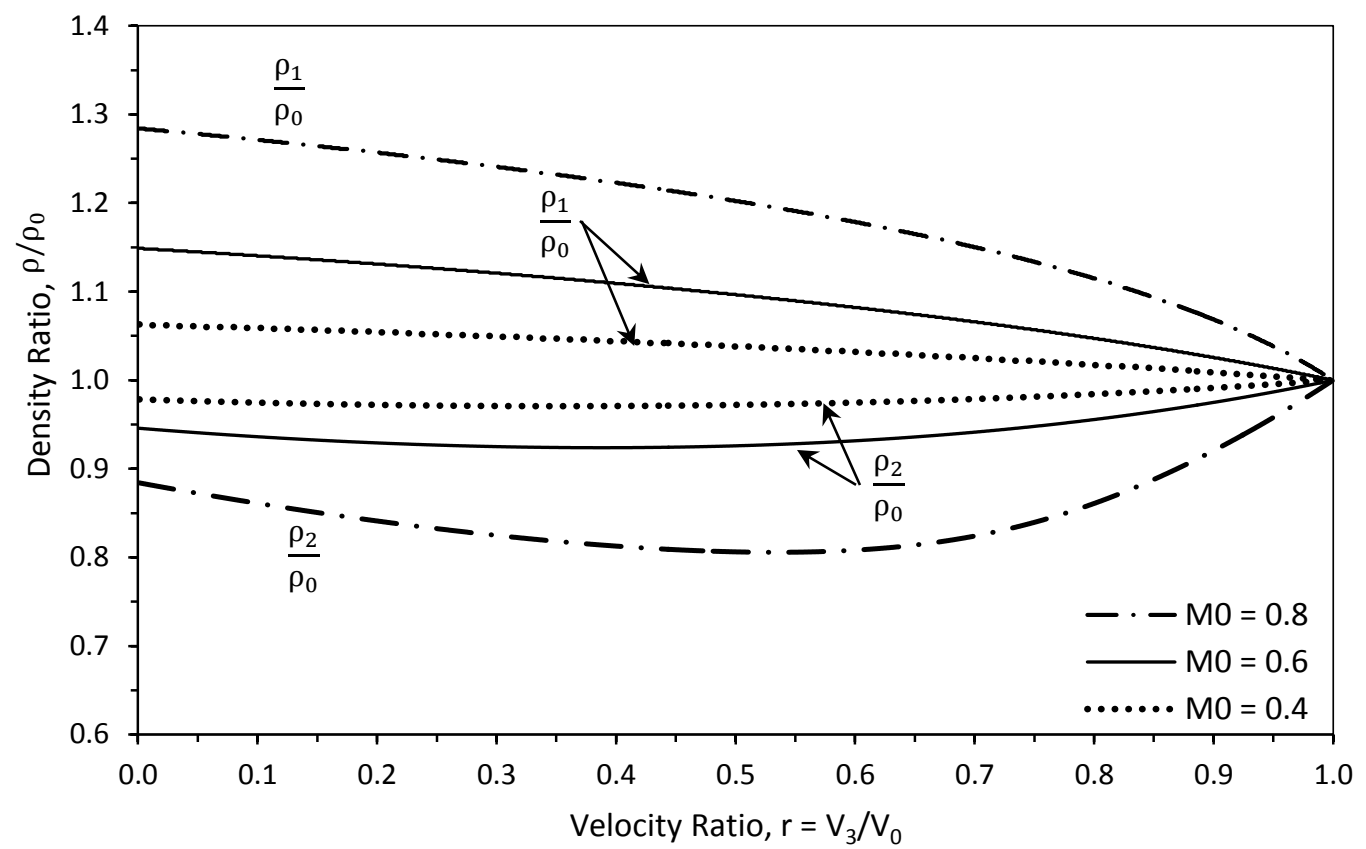

Figure 55. Comparison of the densities immediately before and after the bare turbine for varying free-stream Mach numbers.

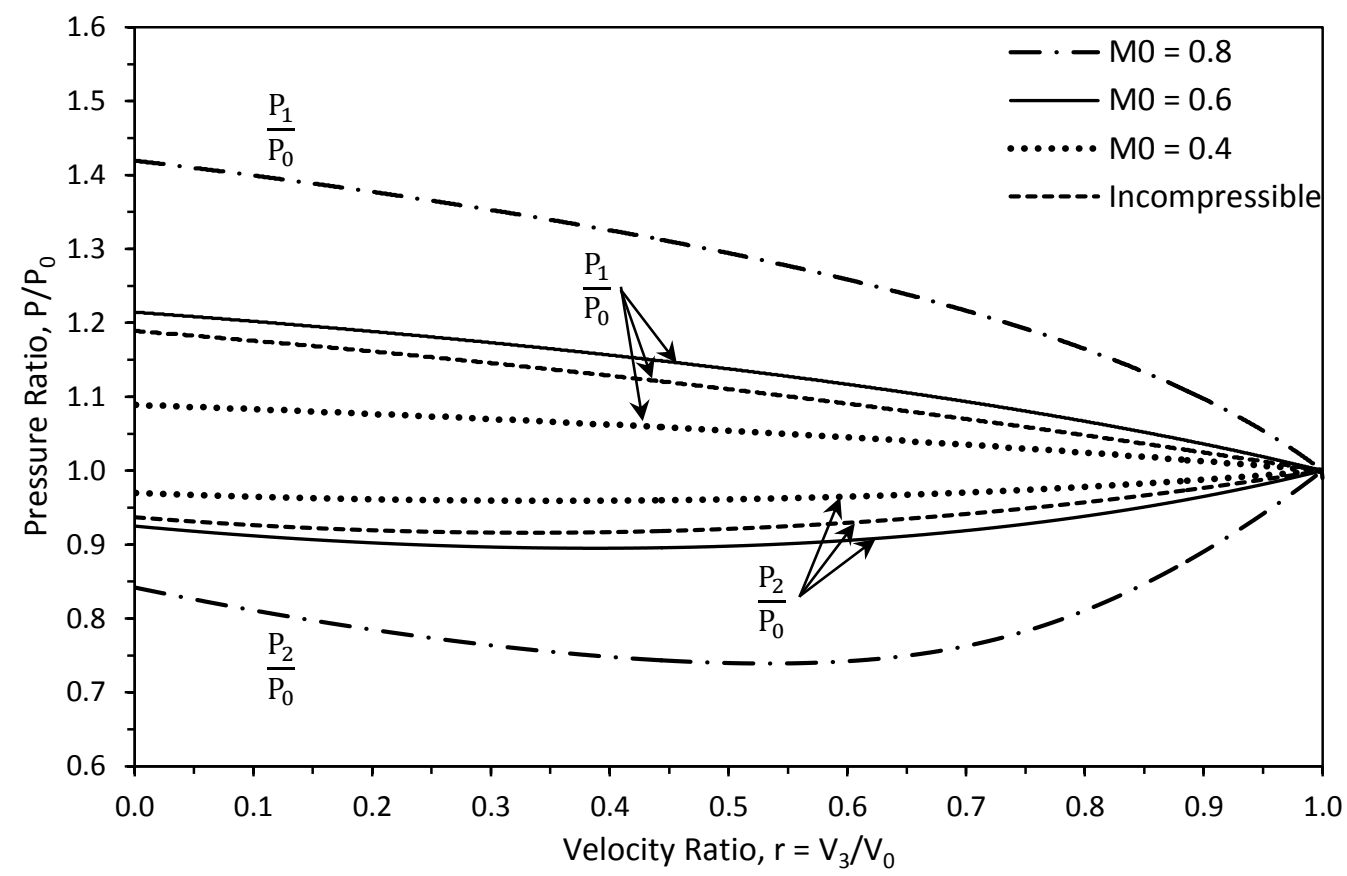

Figure 56. Comparison of the pressures immediately before and after the bare turbine for compressible flow and incompressible flow of varying free-stream Mach numbers. 


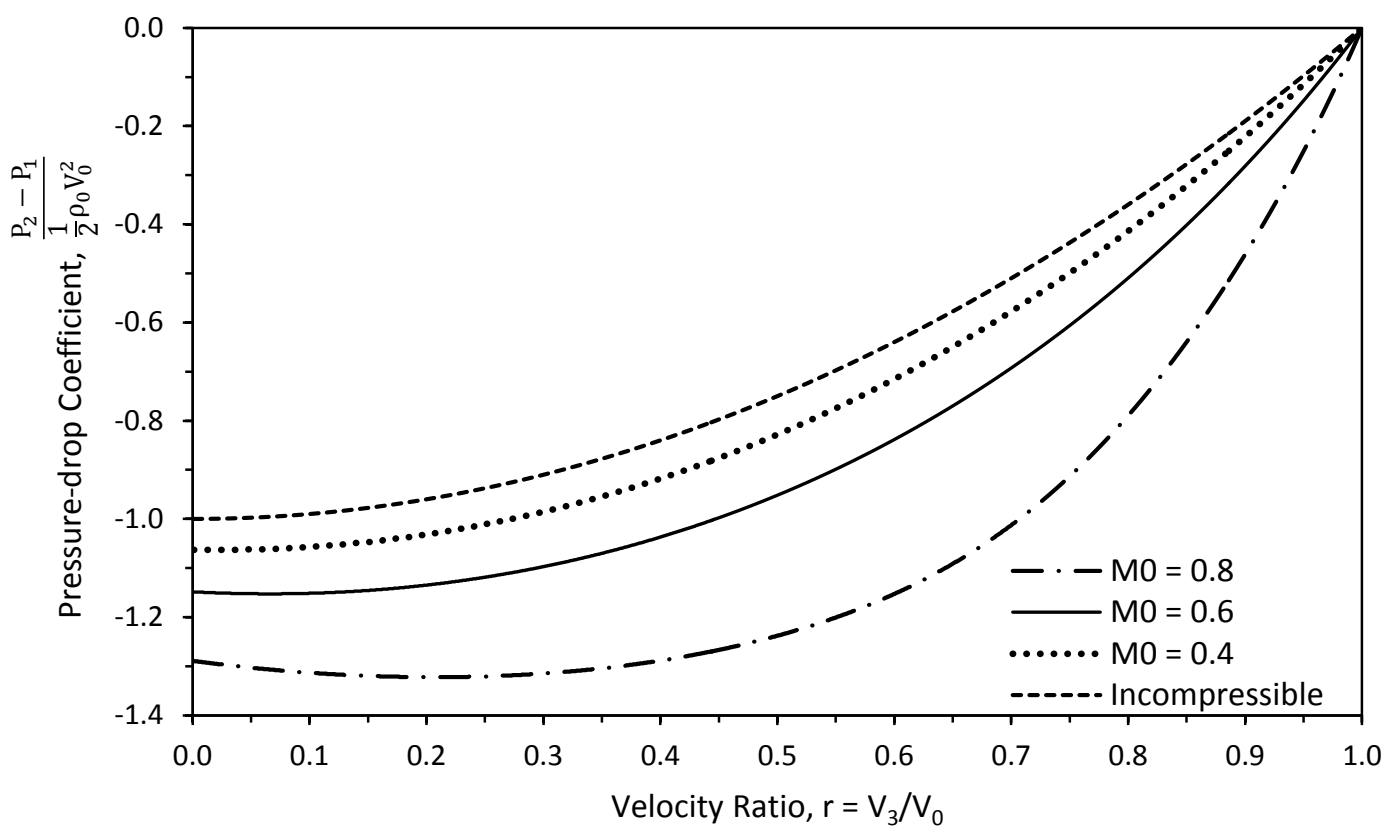

Figure 57. Comparison of the pressure drop across the bare turbine for incompressible flow and compressible flow of varying free-stream Mach numbers.

\subsubsection{Discussion}

The plots in the previous section are discussed below in the order that they appear starting with the power extraction efficiency and the Betz limit for the compressible bare turbine. Note that when the velocity ratio $\mathrm{r}$ is 1 , the flow variables immediately before the disk and in the slipstream are equal to those of the free-stream and the pressure drop across the disk is zero since no power extraction is being made by the turbine.

\section{Power Extraction Efficiency and the Betz Limit for the Compressible, Bare Turbine}

The Betz limit is the maximum percentage of power that a turbine can theoretically capture from the total kinetic energy of the flow. For incompressible flow, the Betz limit and the corresponding velocity ratio $\mathrm{r}$ was determined algebraically in Chapter 1 by differentiating the power extraction efficiency with respect to $r$ and setting the derivative equal to zero. For compressible flow, however, the Betz limit cannot be solved in closed-form due to the implicit nature of the compressible-flow equations and is therefore determined numerically. 
Figure 44 shows that the bare turbine has a slightly higher power extraction efficiency at small values of $\mathrm{r}$ for compressible flow than for incompressible flow. Figure 45 further shows that as the free-stream Mach number $M_{0}$ increases, the Betz limit slightly increases from the well-known value of 0.593 for incompressible flow and occurs at a lower velocity ratio $r$ than that of incompressible flow. This trend is also observed in Figure 46 where the maximum power extraction efficiency increases with increasing $\mathrm{M}_{0}$. Likewise, Figure 47 shows that as the maximum power extraction efficiency increases, the velocity ratio $r$ at which it is achieved decreases.

The slight increase in the Betz limit and the decrease in the corresponding velocity ratio $r$ with the higher $\mathrm{M}_{0}$ are shown to be different to the third decimal place in Table 3. When comparing between incompressible and a $\mathrm{M}_{0}$ of 0.8 , the Betz limit roughly increased by 0.021 while the corresponding velocity ratio $\mathrm{r}$ decreased by 0.036 .

\section{Total Drag Coefficient}

For small velocity ratios $r$, a slight increase in the total drag coefficient with increasing $M_{0}$ can be observed in Figure 48 and Figure 49. As $r$ approaches 1, however, the slight increase in the total drag coefficient becomes negligible.

\section{Mass Flowrate}

The dimensionless mass flowrate has a trend consistent to that of the total drag coefficient. Figure 50 shows that the dimensionless mass flowrate slightly increases as $\mathrm{M}_{0}$ increases for small values of $r$ but becomes indistinguishable between varying $M_{0}$ as $r$ approaches 1 .

\section{Area Ratios}

For small values of r, Figure 51 and Figure 52 shows a slight difference in the ratios of the far

upstream and downstream areas to the disk area $\left(\frac{\mathrm{A}_{0}}{\mathrm{~A}_{\text {disk }}}\right.$ and $\left.\frac{\mathrm{A}_{3}}{\mathrm{~A}_{\text {disk }}}\right)$ not only between compressible and incompressible flow but also between varying $\mathrm{M}_{0}$ for compressible flow. Given the same disk area $A_{\text {disk }}$, the far upstream area $A_{0}$ and the far downstream area $A_{3}$ are slightly larger for 
compressible flow than for incompressible flow. The same areas are also larger for a higher $\mathrm{M}_{0}$ for compressible flow. This observation is consistent with the statements made for the Betz limit and the total drag coefficient. At any given $r$, the power extraction efficiency in equation 4-8 and

the total drag coefficient in equation 4-9 varies only with $\frac{A_{0}}{A_{\text {disk }}}$, and since $\frac{A_{0}}{A_{\text {disk }}}$ is slightly greater for a higher $\mathrm{M}_{0}$, the power extraction efficiency and the total drag coefficient follows the same trend. Note that for $\mathrm{r}$ equal to $1, \mathrm{~A}_{0}$ and $\mathrm{A}_{3}$ are equal to the disk area since no power is being extracted by the turbine.

\section{Flow Variables Immediately Before and After the Disk}

Figure 53to Figure 57 show that there is a rise in velocity and thus the Mach number but a drop in density and pressure across the disk for compressible flow and that the difference in the flow variables immediately before and after the disk is greater for the higher $\mathrm{M}_{0}$. Note that the Mach after the disk is greater than the free-stream Mach when $\mathrm{M}_{0}$ is equal to 0.8 for $\mathrm{r}$ roughly greater than 0.52 as shown in Figure 53. This is also true for the velocity after the disk for $r$ roughly greater than 0.6 as seen in Figure 54.

\section{Compressible and Incompressible Flow}

The compressible- and incompressible-flow solutions for the bare turbine are compared for the same free-stream velocity $V_{0}$, which is equivalent to Mach 0.6 at sea level static conditions. For small values of $r$, the power extraction efficiency, the total drag coefficient, and the dimensionless mass flowrate are slightly greater for compressible flow than for incompressible flow, although the difference becomes negligible as $\mathrm{r}$ approaches unity. Figure 57 also shows that the pressure drop across the disk is also greater for compressible flow than for incompressible flow. In the next section, the compressible Actuator Disk Theory will be extended to the ducted case of the turbine. 


\subsection{Compressible, Ducted Turbine}

The following section will focus on the compressible ducted turbine. Note that the establishment of the performance parameters and the method of solution for the ducted turbine are very much similar if not the same to what was done for the bare turbine. The power extracted by the turbine $\mathrm{P}_{\text {extracted, }}$, the total drag exerted by the flow $\mathrm{D}_{\text {total }}$, the drag experienced by the turbine at the disk plane $\mathrm{D}_{\mathrm{turb}}$, the power extraction efficiency, and the total drag coefficient established for the bare turbine in equations 4-22 to 4-27 also apply to the ducted turbine. However due to the duct enclosing the turbine, the total drag exerted by the flow on the duct-turbine assembly is now the difference of the drag experienced by the turbine and the lip thrust exerted by the duct inlet on the flow

$$
\mathrm{D}_{\text {total }}=\mathrm{D}_{\text {turb }}-\mathrm{T}_{\text {lip }}
$$

Next, the alternative forms of the power extraction efficiency and the total drag coefficient in equations 4-26 and 4-27 are further simplified. By asserting that $A_{3}=A_{\text {disk }}$ and $\rho_{0}=\rho_{3}$ to the conservation of mass between the far upstream and the far downstream stations

$$
\rho_{0} A_{0} V_{0}=\rho_{3} A_{3} V_{3}
$$

the ratio of the free-stream area to the disk area $\frac{A_{0}}{A_{\text {disk }}}$ is found

$$
\frac{A_{0}}{A_{\text {disk }}}=\frac{V_{3}}{V_{0}}=r
$$

By substituting equation 4-30 into 4-26 and 4-27, the power extraction efficiency and the total drag coefficient are now only in terms of the velocity ratio $r$

$$
\begin{aligned}
\eta_{\text {turb }} & =\frac{\mathrm{v}_{3}}{\mathrm{v}_{0}}\left(1-\left(\frac{\mathrm{v}_{3}}{\mathrm{v}_{0}}\right)^{2}\right) \\
\mathrm{C}_{\mathrm{D}} & =2 \frac{\mathrm{v}_{3}}{\mathrm{v}_{0}}\left(1-\frac{\mathrm{v}_{3}}{\mathrm{v}_{0}}\right)
\end{aligned}
$$

Note that this form of the power extraction efficiency and the total drag coefficient are specific to the ducted turbine since the relation between $\frac{A_{0}}{A_{\text {disk }}}$ and $r$ in equation 4-30 is not true for the bare turbine. This form of $\eta_{\text {turb }}$ and $C_{D}$ is also the same for both the compressible and incompressible 
ducted turbine. The complete set of governing equations for the compressible ducted turbine are summarized below.

4.3.1. Summary of Governing Equations

$$
\begin{aligned}
& \mathrm{V}_{0}=\mathrm{M}_{0}\left(\gamma \cdot \frac{\mathrm{P}_{0}}{\rho_{0}}\right)^{\frac{1}{2}} \\
& \frac{A_{\text {disk }}}{A_{0}}=\frac{M_{0}}{M_{1}}\left(\frac{1+\frac{\gamma-1}{2} M_{1}^{2}}{1+\frac{\gamma-1}{2} M_{0}^{2}}\right)^{\frac{\gamma+1}{2(\gamma-1)}} \\
& \frac{\mathrm{P}_{1}}{\mathrm{P}_{0}}=\left(\frac{1+\frac{\gamma-1}{2} \mathrm{M}_{0}^{2}}{1+\frac{\gamma-1}{2} \mathrm{M}_{1}^{2}}\right)^{\frac{\gamma}{\gamma-1}} \\
& \frac{\rho_{1}}{\rho_{0}}=\left(\frac{\mathrm{P}_{1}}{\mathrm{P}_{0}}\right)^{\frac{1}{\gamma}} \\
& \frac{\mathrm{V}_{1}}{\mathrm{~V}_{0}}=\frac{\rho_{0}}{\rho_{1}} \frac{\mathrm{A}_{0}}{\mathrm{~A}_{\mathrm{disk}}} \\
& \mathrm{M}_{3}=\mathrm{V}_{3}\left(\gamma \cdot \frac{\mathrm{P}_{0}}{\rho_{0}}\right)^{-\frac{1}{2}} \\
& \dot{\mathrm{m}}=\rho_{1} \mathrm{~A}_{\text {disk }} \mathrm{V}_{1} \\
& \mathrm{P}_{\text {extracted }}=\dot{\mathrm{m}} \frac{\mathrm{V}_{0}^{2}-\mathrm{V}_{3}^{2}}{2} \\
& \mathrm{D}_{\text {total }}=\dot{\mathrm{m}}\left(\mathrm{V}_{0}-\mathrm{V}_{3}\right) \\
& \mathrm{D}_{\text {turb }}=\dot{\mathrm{m}}\left(\mathrm{V}_{1}-\mathrm{V}_{2}\right)+\mathrm{A}_{\text {disk }}\left(\mathrm{P}_{1}-\mathrm{P}_{2}\right) \\
& \mathrm{T}_{\text {lip }}=\mathrm{D}_{\text {turb }}-\mathrm{D}_{\text {total }} \\
& \frac{\mathrm{T}_{\text {lip }}}{\frac{1}{2} \rho_{0} \mathrm{~V}_{0}^{2} \mathrm{~A}_{\text {disk }}}=2\left(\frac{\mathrm{V}_{1}}{\mathrm{~V}_{0}}\right)\left(1-\frac{\mathrm{V}_{1}}{\mathrm{~V}_{0}}\right)\left(1+\frac{\gamma-1}{2} \mathrm{M}_{0}^{2}\left(\left(\frac{\mathrm{V}_{1}}{\mathrm{~V}_{0}}\right)^{2}-1\right)\right)^{\frac{1}{\gamma-1}} \\
& +\frac{2}{\gamma \mathrm{M}_{0}^{2}}\left(\left(1+\frac{\gamma-1}{2} \mathrm{M}_{0}^{2}\left(\left(\frac{\mathrm{V}_{1}}{\mathrm{~V}_{0}}\right)^{2}-1\right)\right)^{\frac{\gamma}{\gamma-1}}-1\right) \\
& C_{D}=2 \frac{V_{3}}{V_{0}}\left(1-\frac{v_{3}}{V_{0}}\right)
\end{aligned}
$$




$$
\eta_{\text {turb }}=\frac{\mathrm{v}_{3}}{\mathrm{v}_{0}}\left(1-\left(\frac{\mathrm{v}_{3}}{\mathrm{v}_{0}}\right)^{2}\right)
$$

Again, these governing equations provide the necessary system of equations to solve for the flow variables before the disk and throughout the slipstream and the performance of the ducted turbine in terms of the free-stream flow conditions $\gamma, \rho_{0}, P_{0}$, and $V_{0}$ and the disk area $A_{\text {disk }}$.

\subsubsection{Method of Solution}

The method of solution for the ducted turbine is the same as for the bare turbine. With the implications of the constant duct area given in equation 2-76 to 2-79, the unknown variables to determine for the ducted turbine are reduced to the following fourteen variables $A_{0}, M_{0}, M_{1}, \rho_{1}$, $\mathrm{P}_{1}, \mathrm{~V}_{1}, \mathrm{M}_{3}, \mathrm{D}_{\text {turb }}, \mathrm{D}_{\text {total }}, \mathrm{T}_{\text {lip }}, \mathrm{P}_{\text {extracted }}, \mathrm{C}_{\mathrm{D}}, \eta_{\text {turb }}$ and $\dot{\mathrm{m}}$. These fourteen unknown variables are solved in Engineering Equation Solver (EES) for a given $\gamma, \rho_{0}, P_{0}$, and $A_{\text {disk }}$ in terms of the velocity ratio $r$ ranging from 0.001 to 1 for varying values of $M_{0}$, as was done for the bare turbine, using the fourteen non-linear coupled governing equations summarized in section 4.3.1. Lastly, the solutions are made dimensionless by the free-stream flow conditions and the disk area giving $\frac{\rho_{1}}{\rho_{0}}, \frac{\mathrm{P}_{1}}{\mathrm{P}_{0}}, \frac{\mathrm{V}_{1}}{\mathrm{~V}_{0}}, \frac{\mathrm{M}_{1}}{\mathrm{M}_{0}}, \frac{\mathrm{A}_{0}}{\mathrm{~A}_{\text {disk }}}, \frac{\dot{\mathrm{m}}}{\rho_{0} \mathrm{~V}_{0} \mathrm{~A}_{\text {disk }}}$, and $\frac{\mathrm{P}_{2}-\mathrm{P}_{1}}{\frac{1}{2} \rho_{0} \mathrm{~V}_{0}^{2}}$. In addition, the lip thrust and the turbine drag are also nondimensionlized by the reference force which introduces the lip thrust and the turbine drag coefficients, $\frac{T_{\text {lip }}}{\frac{1}{2} \rho_{0} A_{\text {disk }} V_{0}^{2}}$ and $\frac{D_{\text {turb }}}{\frac{1}{2} \rho_{0} A_{\text {disk }} V_{0}^{2}}$, respectively. These dimensionless parameters are presented in the next section.

\subsubsection{Results}

The compressible-flow solutions for the ducted turbine are determined for the same disk area and free-stream conditions as the bare turbine, which are given in Table 1. The compressible-flow solutions are again obtained for $\mathrm{M}_{0}$ of $0.4,0.6$, and 0.8 , while the incompressible-flow solutions are obtained for $\mathrm{V}_{0}$ equivalent to Mach 0.6 at sea level static conditions. The power extraction 
efficiency, the total drag coefficient, the dimensionless mass flowrate, the ratios of the far upstream area to the disk area, and the dimensionless flow variables immediately before the disk are plotted against the velocity ratio $\mathrm{r}$ for $\mathrm{M}_{0}$ of $0.4,0.6$, and 0.8 in Figure 58 to Figure 66 . When applicable, the dimensionless incompressible-flow solutions are also included in the plots for comparison purposes. These plots are discussed in the next section.

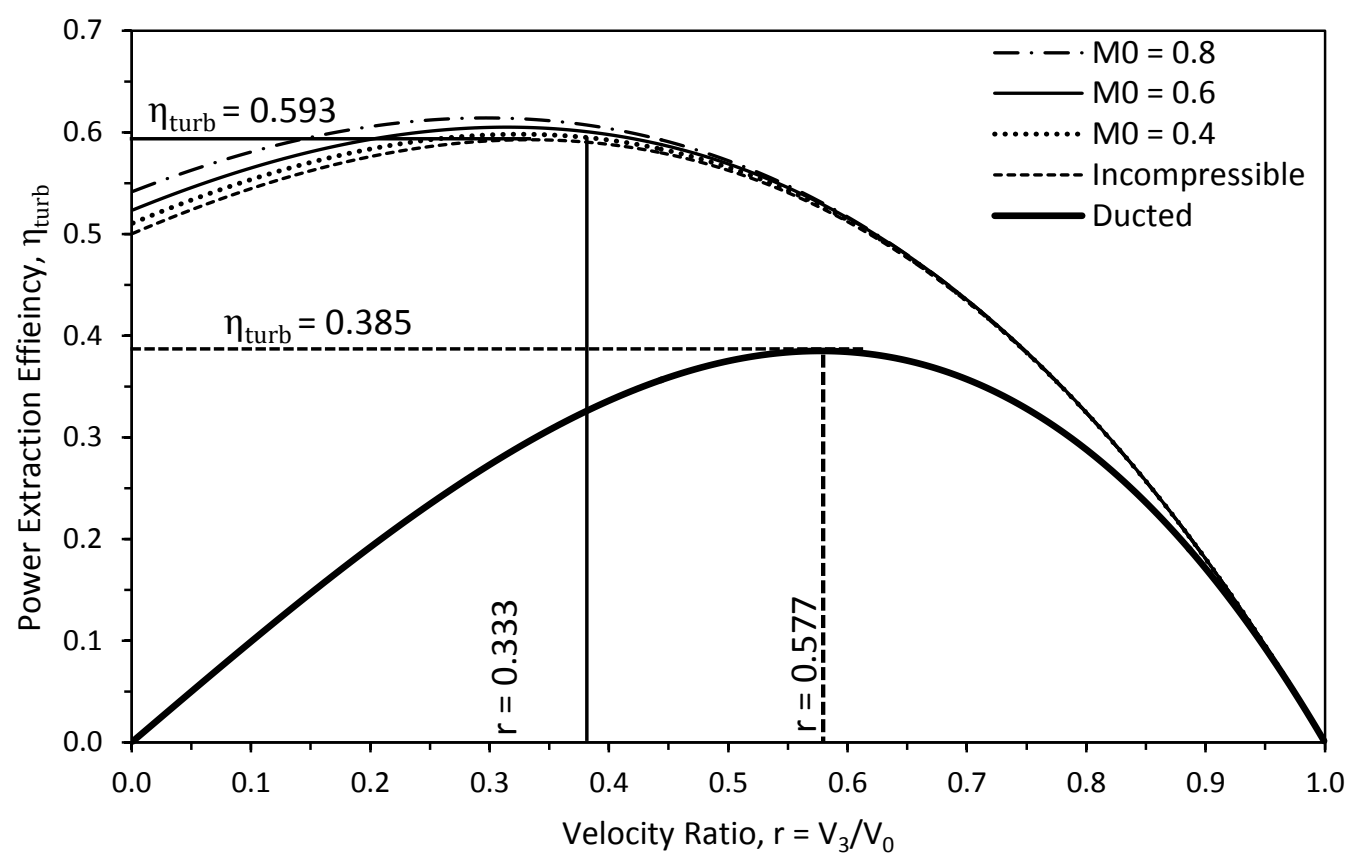

Figure 58. Comparison of the power extraction efficiency between the bare and the ducted turbine for incompressible flow and compressible of varying free-stream Mach numbers. 


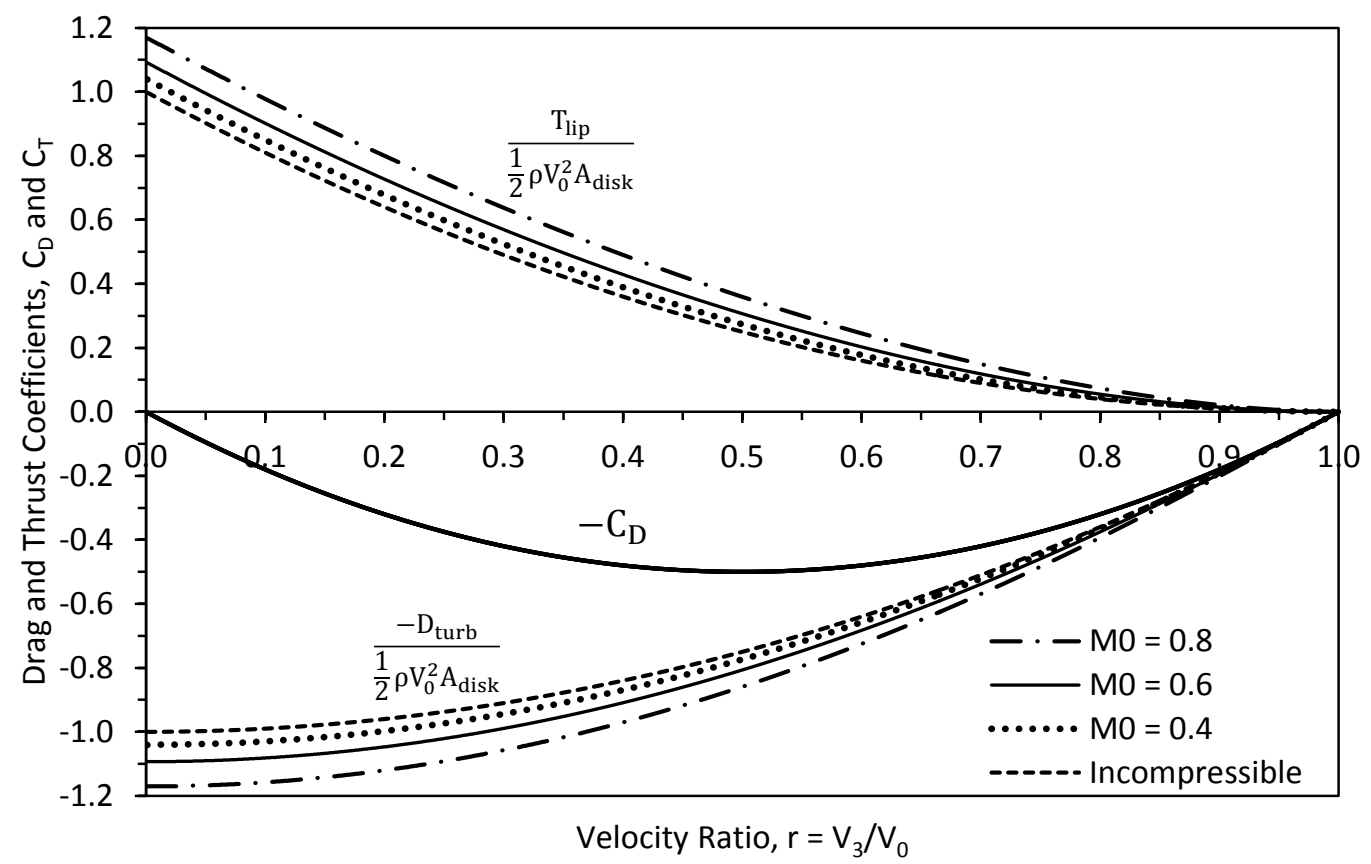

Figure 59. Contribution of the turbine drag and the lip thrust to the total drag for incompressible flow and for compressible flow of varying free-stream Mach numbers. Note that the total drag and the turbine drag are made negative to signify that they are in the opposite direction of the flow.

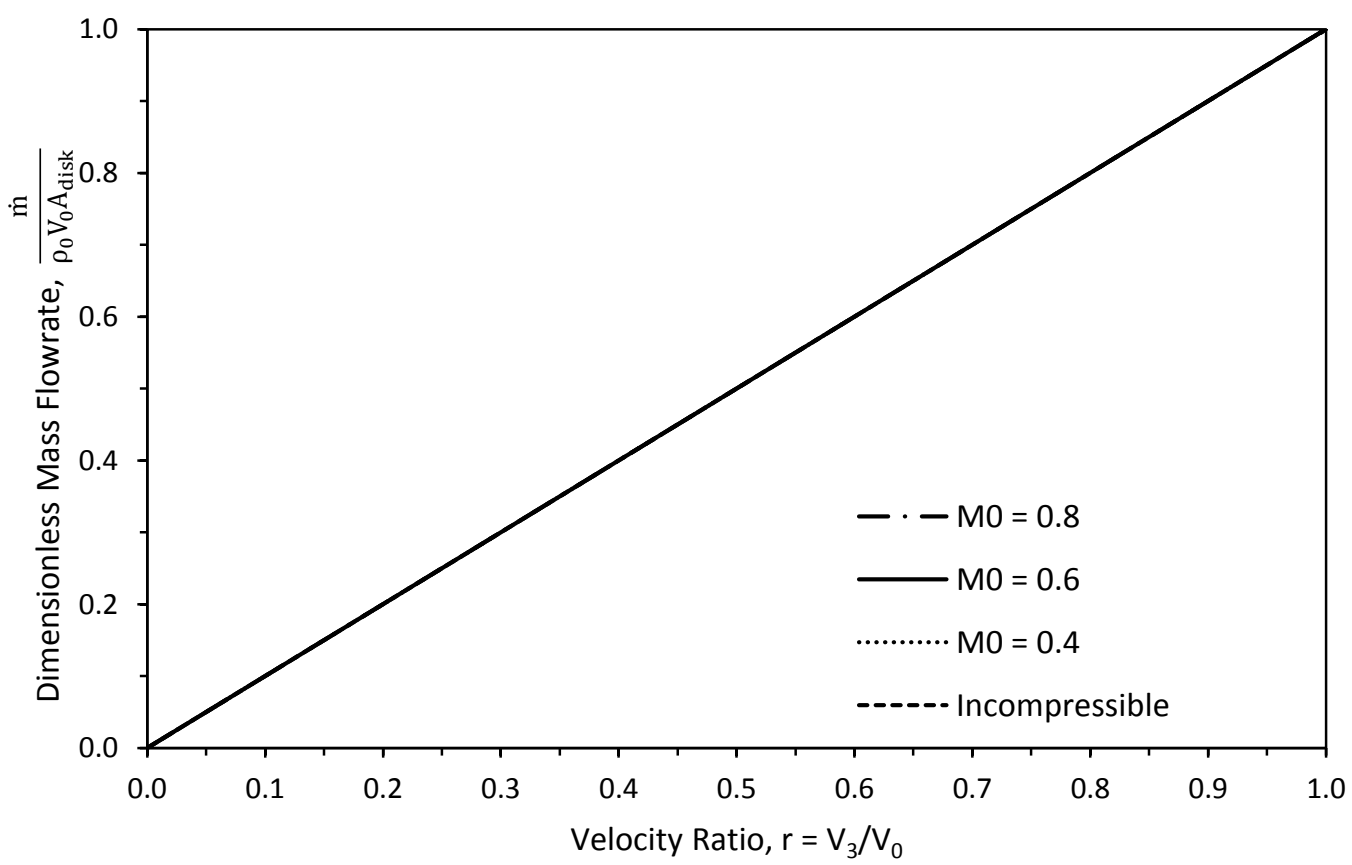

Figure 60. Comparison of the dimensionless mass flowrate of the ducted turbine between incompressible flow and compressible flow of varying free-stream Mach numbers. 


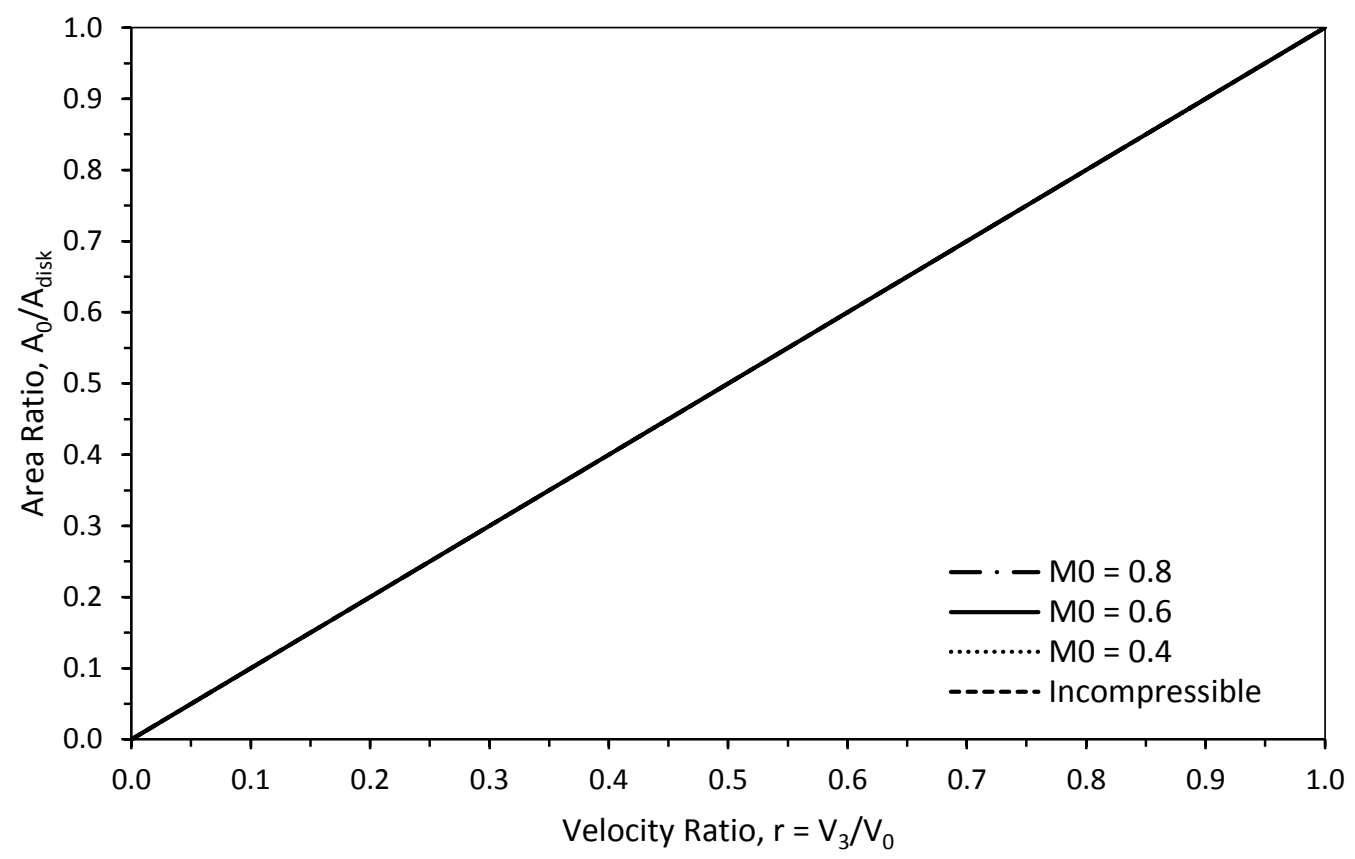

Figure 61. Comparison of the cross-sectional free-stream area of the ducted turbine for incompressible flow and compressible flow of varying free-stream Mach numbers.

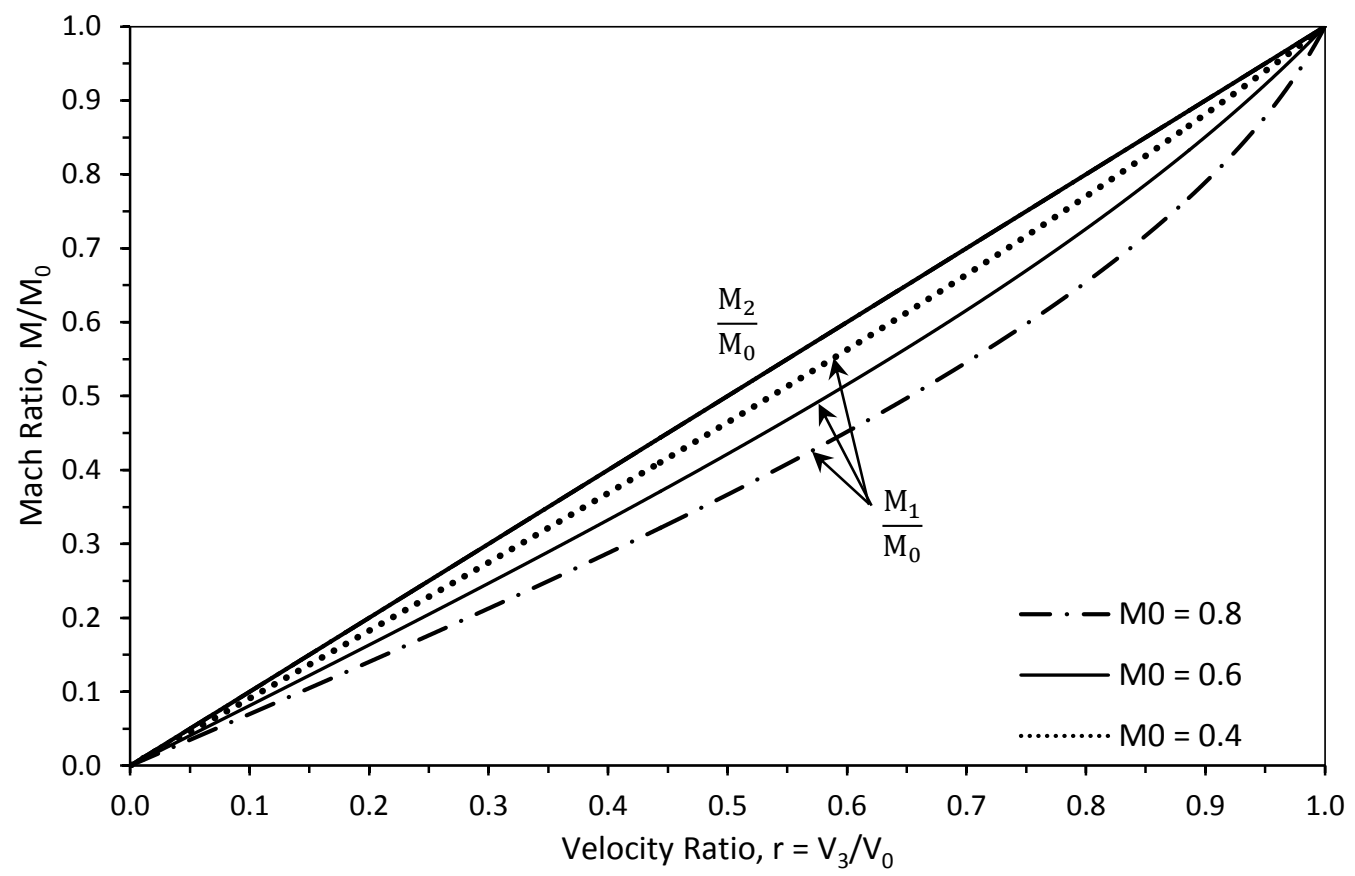

Figure 62. Comparison of the Mach numbers immediately before the ducted turbine for varying free-stream Mach numbers. Note that $\mathbf{M}_{2}=\mathbf{M}_{3}$. 


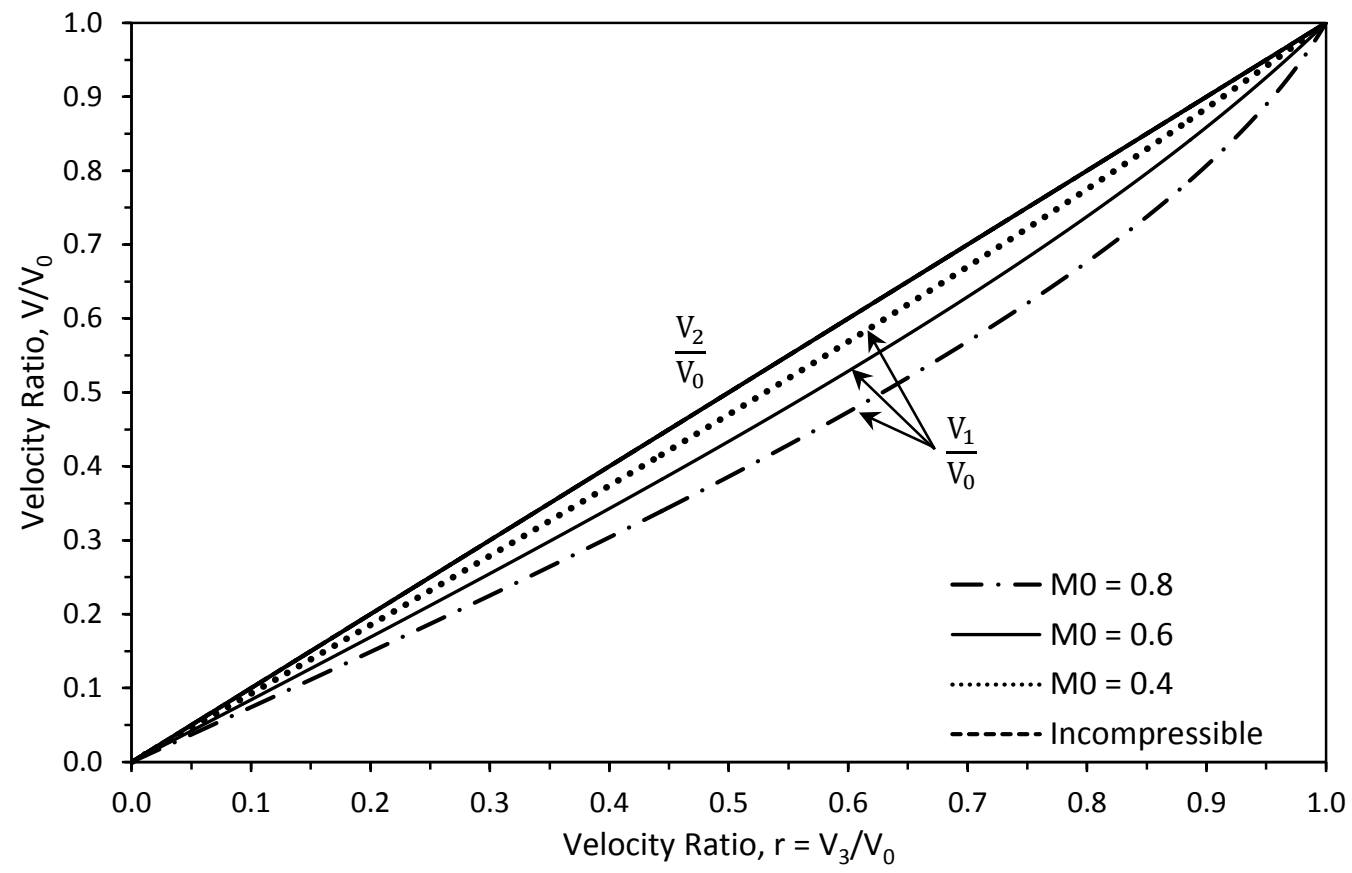

Figure 63. Comparison of velocities immediately before the ducted turbine for incompressible flow and compressible flow of varying free-stream Mach numbers. Note that $\mathbf{V}_{\mathbf{2}}=\mathbf{V}_{\mathbf{3}}$.

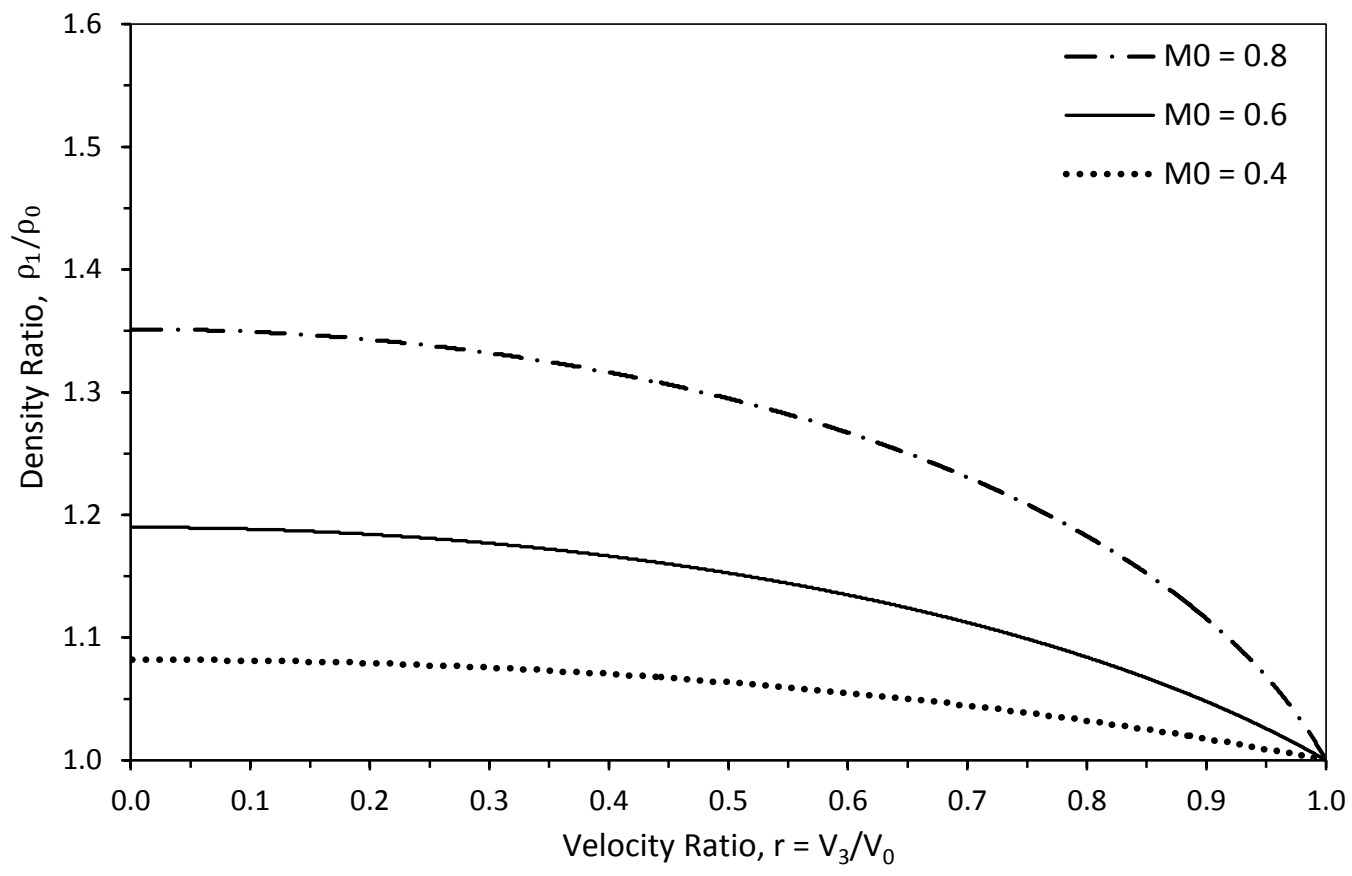

Figure 64. Comparison of the densities immediately before the ducted turbine for varying freestream Mach numbers. 


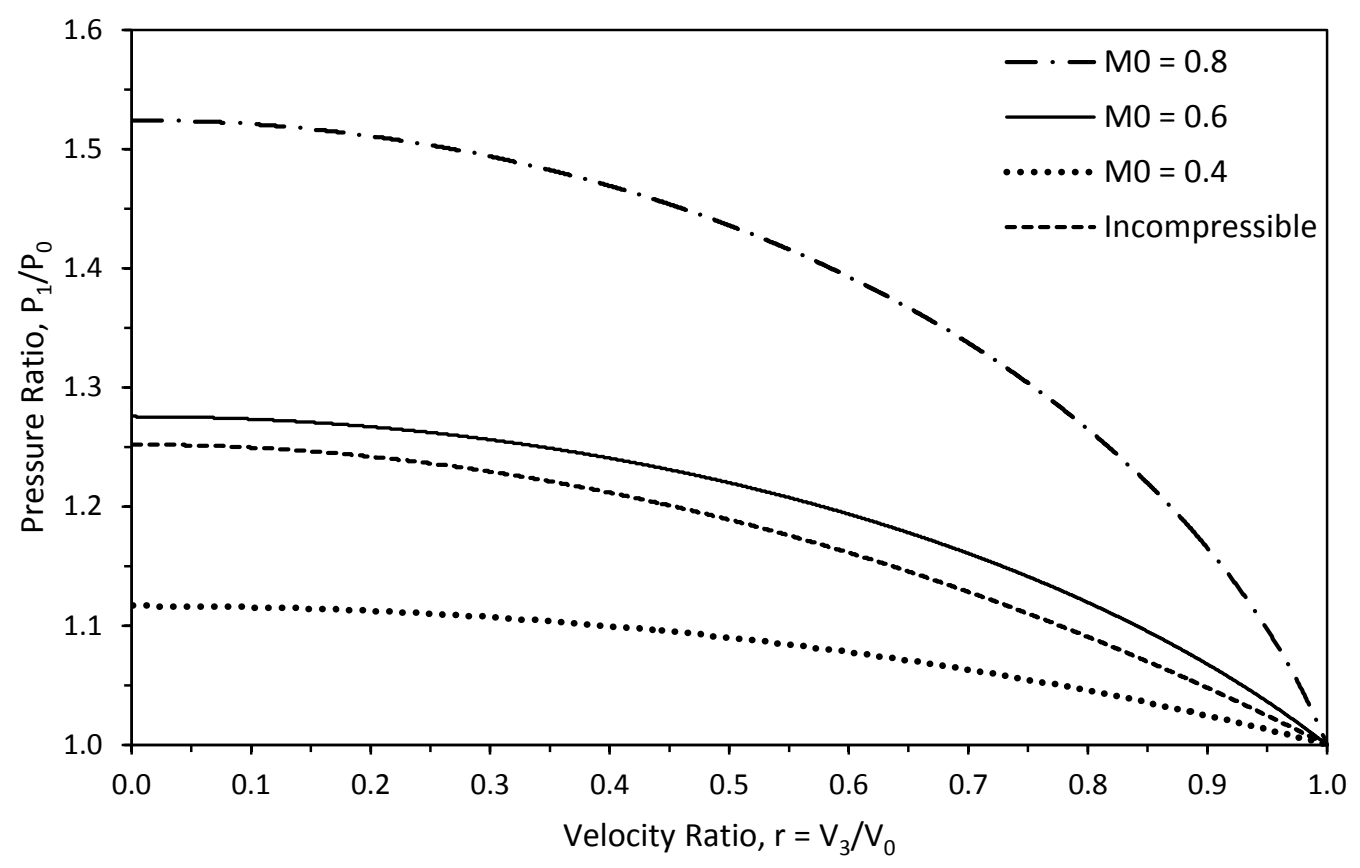

Figure 65. Comparison of the pressures immediately before the ducted turbine for incompressible and compressible flow of varying free-stream Mach numbers.

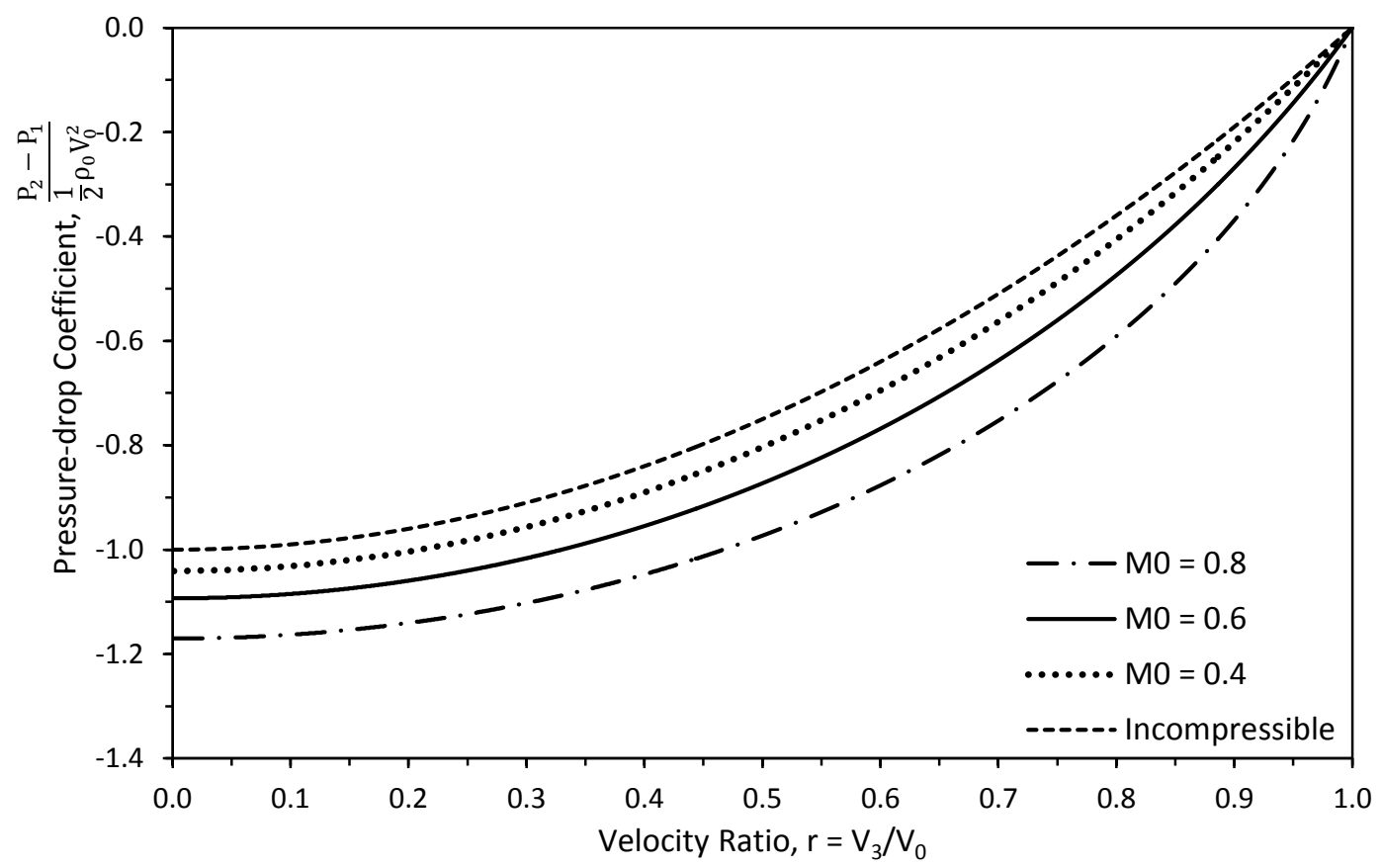

Figure 66. Comparison of the pressure drop across the ducted turbine for incompressible and compressible flow of varying free-stream Mach numbers. 


\subsubsection{Discussion}

The resulting plots are discussed below in the order that they appear beginning the power extraction efficiency and the Betz limit of the ducted turbine. Note that when the velocity ratio $r$ is 1 , the flow variables immediately before the disk are equal to those of the free-stream and the pressure-drop across the disk is zero due to no power extraction by the ducted turbine.

\section{Betz Limit}

Contrary to the observations made for the bare turbine, Figure 58 shows that the power extraction efficiency of the ducted turbine is not affected by the free-stream Mach number $\mathrm{M}_{0}$ and that it is the same for incompressible and compressible flow. This indicates that compressibility and $\mathrm{M}_{0}$ also have no effect on the Betz limit and the velocity ratio $r$ at which it occurs. The power extraction efficiency given in equation 4-46 is differentiated with respect to the velocity ratio $r$ to find the value of the Betz limit and its corresponding r. The resulting Betz limit is 0.385 occurring at a velocity ratio $\mathrm{r}$ of 0.577 . In closed form expression, the Betz limit is $2\left(3^{-\frac{3}{2}}\right)$ while its corresponding $\mathrm{r}$ is $\frac{\sqrt{3}}{3}$. This means that the ducted turbine cannot capture more than $38.5 \%$ of the total kinetic energy in the flow for useful power. Figure 58 shows that the Betz limit is significantly lower and occurs at a higher velocity ratio $r$ for the ducted turbine than for the bare turbine.

\section{Drag and Lip Thrust Coefficient}

Figure 59 shows the total drag coefficient is also not affected by the free-stream Mach number $\mathrm{M}_{0}$ or the compressibility of the flow. This is expected since the total drag coefficient given in equation 4-45 is only dependent on the velocity ratio $r$ and is the same equation for incompressible and compressible flow. However, the individual contribution of the turbine drag and the lip thrust to the total drag differs greatly not only between compressible and incompressible flow but also between varying $\mathrm{M}_{0}$ as evident in Figure 59. The difference 
between the lip thrust and the turbine drag is the smallest for incompressible flow while the difference increases with increasing $\mathrm{M}_{0}$ for compressible flow.

\section{Mass Flowrate}

Consistent to the trend of the total drag coefficient, Figure 60 shows that the dimensionless mass flowrate is independent of the free-stream Mach number $\mathrm{M}_{0}$ and is the same for incompressible and compressible flow.

\section{Area Ratio}

Likewise, the ratio of the far upstream area to the disk area $\frac{A_{0}}{A_{\text {disk }}}$ is found to be the same for incompressible and compressible flow regardless of the free-stream Mach number $\mathrm{M}_{0}$.

\section{Flow Variables Across the Disk}

When comparing the compressible-flow variables immediately before the disk between varying $\mathrm{M}_{0}$, the pressure and the density increases while the velocity and thus the Mach number decreases with increasing $M_{0}$ as evident in Figure 62 to Figure 66 given the same free-stream pressure and density. Note that the flow variables immediately after the disk are equal to the flow variables at the far downstream due the constant duct area in the slipstream. As a result, the ratio of the velocity after the disk to the free-stream velocity is the same for compressible and incompressible flow regardless of $\mathrm{M}_{0}$. When comparing the compressible- and incompressible-flow solutions of the same $V_{0}$, the pressure-drop across the disk is greater for compressible flow than for incompressible flow for all velocity ratios $r$ as shown in Figure 66. 


\subsection{Chapter Summary}

In closing remarks, given the same free-stream density and pressure and the disk area, the bare turbine achieves a slightly higher extraction efficiency — and total drag — for compressible flow than for incompressible flow. When considering compressible flow, the maximum extraction efficiency or the Betz limit is found to slightly increase while the velocity ratio $r$ at which it occurs decreases with increasing free-stream Mach numbers $M_{0}$. This means that the compressible bare turbine can theoretically extract slightly more useful energy from the total kinetic energy of the free-stream flow when the free-stream flow has a higher $\mathrm{V}_{0}$. The bare turbine can also extract more energy from the free-stream flow than the ducted turbine of the same disk area. In fact, the maximum percentage of power that can theoretically be extracted by the bare turbine from the total kinetic energy of the flow is significantly greater than that of the ducted turbine. The Betz limit of the ducted turbine and the velocity ratio $r$ at which it occurs is a constant regardless of the compressibility of the flow. 


\section{CONCLUSION AND RECOMMENDATIONS}

The classical Actuator Disk Theory, also known as Froude's Momentum Theory, is a theory that determines the ideal performance of an energy-adding or an energy-extracting device such as a propeller or a turbine by applying the conservation of mass, momentum, and energy to a flow stream that is considered steady, inviscid, one-dimensional, and incompressible. Because compressibility effects arise in real applications of such devices, the current thesis extends the classical Actuator Disk Theory into the regime of subsonic compressible flow. The specific motivation for the present work is to develop a mathematical model that predicts the performance limits of a small ram-air turbine (microRAT) [4] operating at high subsonic Mach numbers for the application of the Boundary Layer Data System (BLDS). Developed by Dr. Russell Westphal and his team of students, the BLDS is a fully autonomous system that measures the flow properties of the boundary layer at the surface of an aircraft in flight. Because the electronic components of the BLDS are not rated to perform below $-20^{\circ} \mathrm{C}$, their performance becomes unreliable during test flights at attitudes between 30,000 and 40,000 feet where the air temperature ranges from $-40^{\circ} \mathrm{C}$ to $-57^{\circ} \mathrm{C}$ or below. The concept of utilizing a small ram-air turbine (microRAT) to provide power to a heating element that maintains operable temperatures inside the BLDS has previously been investigated by Victor Villa [2]. The goal of the current thesis is to develop the compressible Actuator Disk Theory using the three laws of conservation and isentropic thermodynamics to provide the necessary equations to predict the ideal performance of the microRAT.

The governing equations of the compressible Actuator Disk Theory were established for two cases: the unducted (bare) and the ducted. The unducted (bare) case considered the actuator disk as the only assembly within the flow stream, while the ducted case considered the disk as enclosed by a duct of the same cross-sectional area as of the disk. The compressible-flow governing equations were then applied to determine the ideal performance of a propeller and a turbine for both the bare and ducted cases. The application of the compressible Actuator Disk 
Theory to the bare propeller has already been investigated by Delano and Crigler [5] but was included in the current thesis to show agreement and to build confidence in the results of the bare and ducted turbine in compressible flow, which was the main focus of the current thesis.

The governing equations of both the classical and the compressible Actuator Disk Theory provide a system of equations to determine the performance of the actuator disk and the flow variables and the cross-sectional area at each of the four stations throughout the streamtube in terms of the disk area and the free-stream flow conditions. It was shown in Chapter 1 that the unknown variables within the governing equations of the classical Actuator Disk Theory can be made dimensionless solely in terms of the given inputs and be solved explicitly. For the compressible Acutator Disk Theory, some of the unknown variables within the governing equations cannot be isolated or be expressed as a function of only the independent variables and thus have to be solved implicitly using a numerical method. As a result, the governing equations for the compressible theory have a mixture of both the inputs and the outputs and thus cannot cleanly be presented as dimensionless quantities. Therefore, the governing equations were derived in dimensional forms, and the solutions were presented in dimensionless forms.

Compressible-flow and incompressible-flow solutions were obtained for the bare and ducted cases of the propeller and the turbine for an actuator disk cross-sectional area of $1 \mathrm{ft}^{2}$ and the freestream pressure, density, and specific heat ratio at sea level static conditions. The compressibleflow solutions were determined for varying values of the free-stream Mach number while, for comparison, the incompressible-flow solutions were determined for a free-steam velocity equivalent to one of the various free-stream Mach numbers chosen for the compressible-flow solutions. All of the solutions were made dimensionless by the disk area and the free-stream flow conditions. The following conclusions were found for the ducted and bare cases of the energyadding device or the propeller. 


\subsection{Propeller - Energy-addition}

1) When considering the case of the compressible bare propeller, the maximum power coefficient that is required for the flow to be sonic immediately before the disk decreases rapidly with increasing free-stream Mach number $\mathrm{M}_{0}$.

2) The propulsive efficiency is approximately the same for compressible and incompressible flow for high subsonic free-stream Mach numbers $\mathrm{M}_{0}$ because the flow before the disk becomes sonic at lower power coefficients so that negligible change can be observed in the efficiency, which agrees with what was concluded by Delano and Crigler [5]. However, the propulsive efficiency of the bare propeller differs very slightly between compressible and incompressible flow at very large power coefficients for low subsonic free-stream Mach numbers. When looking at the case for $\mathrm{M}_{0}$ of 0.2 at a maximum power coefficient of 58.7, a 0.005 increase in the propulsive efficiency is observed between the compressible and incompressible flow. Since the equation for the propulsive efficiency is dependent only on $\frac{\mathrm{V}_{3}}{\mathrm{~V}_{0}}$, and if the propulsive efficiency for the bare propeller is the same for compressible and incompressible flow, then logically the same conclusion can be made for the velocity ratio $\frac{V_{3}}{V_{0}}$. In addition, since the equation for $\frac{V_{3}}{V_{0}}$ only depends on the area ratio $\frac{A_{0}}{A_{\text {disk }}}$ for a given power coefficient, the same conclusion can also be said for

$\frac{A_{0}}{A_{\text {disk }}}$. It was found that there is a 0.038 difference in $\frac{A_{0}}{A_{d i s k}}$ and a 0.091 difference in $\frac{V_{3}}{V_{0}}$ between compressible and incompressible flow for $\mathrm{M}_{0}$ of 0.2 with a maximum power coefficient of 58.7, further showing that the propulsive efficiency for the bare propeller is not be the same for compressible and incompressible flow. However, such power coefficients are impractical in real life applications of propellers. The same conclusion is true for the total thrust coefficient of the compressible bare propeller. The total thrust coefficient is the same for compressible and incompressible flow for high subsonic free- 
stream Mach numbers $\mathrm{M}_{0}$ but is observed to differ very slightly at large power coefficients for low subsonic $\mathrm{M}_{0}$.

3) The ducted propeller has the same propulsive efficiency in both compressible and incompressible flow at given power coefficients, regardless of the free-stream Mach number.

4) The total thrust coefficient of the ducted propeller is the same for compressible and incompressible flow, although the individual contributions of the lip thrust and the propeller thrust to the total thrust change with varying $\mathrm{M}_{0}$ for compressible flow. The difference between the lip thrust and the propeller thrust is found to decrease as the freestream Mach number increases for compressible flow, while this difference is the greatest for incompressible flow.

5) When comparing compressible and incompressible flow of the same $V_{0}$, the pressure-rise across the disk is greater for compressible flow than for incompressible flow for both the bare and ducted propeller.

It was found that the results of the bare propeller agree with those obtained by Delano and Crigler [5]. In addition, it was also found that the lip thrust derived from the difference of the total thrust and the disk thrust also agrees with the lip thrust derived by Küchemann and Weber [3]. Such agreements built confidence not only in the current method of solution and the compressible-flow results obtained for the bare and ducted propeller but also in the results that were also found for the compressible bare and ducted turbine, which are presented next. 


\subsection{Turbine - Energy-extraction}

6) The power extraction efficiency and the total drag coefficient of the bare turbine are slightly higher for compressible flow than for incompressible flow. As the free-stream Mach $\mathrm{M}_{0}$ increases, the maximum extraction efficiency or the Betz limit for the compressible bare turbine slightly increases from the incompressible value of 0.593 , while the ratio of the far downstream velocity to the free-stream velocity $r$ at which the Betz limit occurs decreases slightly from the incompressible value of 0.333 . When the free-stream Mach number is increased from incompressible to 0.8 , the Betz limit is found to increase by 0.021 while the corresponding $\mathrm{r}$ decreases by 0.036 , showing that the compressible bare turbine can theoretically extract slightly more useful energy from the free-stream flow when it has a higher velocity.

7) The power extraction efficiency and the Betz limit and its corresponding velocity ratio $r$ of the ducted turbine are not affected by the free-stream Mach number $\mathrm{M}_{0}$ and are found to be the same for incompressible and compressible flow. The Betz limit of the ducted turbine is found to be 0.385 occurring at a velocity ratio $r$ of 0.577 . This Betz limit is 0.208 lower and occurs at a velocity ratio $r$ that is 0.244 higher than that of the incompressible bare turbine.

8) The total drag coefficient of the ducted turbine is also found to be the same for incompressible and compressible flow regardless of the free-stream Mach number $\mathrm{M}_{0}$. While this is true, the individual contribution of the turbine drag and the lip thrust to the total drag differs greatly not only between compressible and incompressible flow but also between varying $\mathrm{M}_{0}$. The difference between the lip thrust and the turbine drag is the smallest for incompressible flow and increases with increasing $\mathrm{M}_{0}$ for compressible flow.

9) The pressure-drop across the disk is greater for compressible flow than for incompressible flow for all velocity ratios $r$ for both the bare and ducted turbine when compared at equal values of the upstream velocity $\mathrm{V}_{0}$. 
Overall, the analysis done in this current thesis shows that compressibility has very little influence on the ideal performance of an actuator disk. Thus, the ideal performance of the small ram-air turbine (mircroRAT), which was the motivation of the current work, can be determined using the classical Actuator Disk Theory without having to worry about the effects of compressibility. 


\subsection{Recommendations}

Based on these conclusions of the current thesis, it is recommended that future work considers implementing important parameters such as rotation in the slipstream and the effect of entropy changes due to profile and shock losses to the compressible Actuator Disk Theory for both the bare and ducted cases. As was done in this current thesis, the compressible theory that accounts for such parameters should be applied to determine the performance of the propeller and the turbine with and without a duct enclosing the actuator disk. When considering the bare and ducted propeller, solutions should also be investigated for a supersonic wake immediately behind the actuator disk assuming that the power addition at the disk plane is greater than what is required to produce sonic flow $\left(\mathrm{M}_{1}=1\right)$ immediately before the disk. In addition, a duct of variable area or various inlet contour would also be useful extensions to the compressible Actuator Disk Theory. 


\section{REFERENCES}

[1] Horlock, J.H. Actuator Disk Theory: Discontinuities in Thermo Fluid Dynamics. London: McGraw-Hill, 1978.

[2] Glauert, H. The Elements of Aerofoil and Airscrew Theory. Cambridge: U, 1943.

[3] Küchemann, Dietrich, and Johanna Weber. Aerodynamics of Propulsion. New York: McGraw-Hill, 1953.

[4] Villa, Victor F. Evaluation of Design Tools for the Micro-Ram Air Turbine. MS Thesis. California Polytechnic State University, San Luis Obispo. 2015.

[5] Delano, James B., and John L. Crigler. "Compressible-Flow Solutions for the Actuator Disk Theory." NACA L53A07 RM (1953).

[6] Greitzer, E. M., Choon Sooi. Tan, and M. B. Graf. Internal Flow: Concepts and Applications. Cambridge, U.K.: Cambridge UP, 2004.

[7] Betz, A. Introduction to the Theory of Flow Machines. Oxford: Pergamon, 1966.

[8] Ames Research Staff. "Equations, Tables, and Charts for Compressible Flow." NACA Report 1135 (1953).

[9] Anderson, John David. Modern Compressible Flow: With Historical Perspective. New York: McGraw-Hill, 2004.

[10] Vogeley, Arthur W. "Axial-Momentum Theory for Propellers in Compressible Flow." NACA TN2164 (1951). 


\section{APPENDICES}

A. Incompressible Bare and Ducted Fan

This appendix will investigate the specific case of the incompressible bare and ducted propeller where the freestream velocity is zero. This specific case corresponds to the applications of a fan since its purpose is to generate a mass flow for a given power input when the freestream fluid is at rest. Therefore, the performance parameters of the incompressible bare and the ducted fan will be established using the governing equations of the classical Actuator Disk Theory given in section 1.2.2 for the bare case and section 1.2.3 for the ducted case, given the following is true

$$
\mathrm{V}_{0}=0
$$

\section{A.1. Bare Fan}

Because the bare fan is simply a specific case of the bare propeller where the freestream velocity is zero, the dimensional performance parameters of the bare propeller can be applied to the case of the bare fan. By asserting that the freestream velocity is zero, the performance parameters from equation 1-58 to $1-62$ can be put in terms of the far downstream velocity as shown

$$
\begin{gathered}
\mathrm{T}_{\text {total }}=\dot{\mathrm{m} V_{3}} \\
\mathrm{~T}_{\text {fan }}=\frac{1}{2} \rho \mathrm{A}_{\text {disk }} \mathrm{V}_{3}^{2} \\
\mathrm{~T}_{\text {total }}=\mathrm{T}_{\text {fan }} \\
\mathrm{P}_{\text {input }}=\frac{1}{2} \dot{\mathrm{m}} \mathrm{V}_{3}^{2} \\
\dot{\mathrm{m}}=\rho \mathrm{A}_{\text {disk }} \frac{\mathrm{V}_{3}}{2}
\end{gathered}
$$

Because a popular figure of merit of a fan is its ability to provide mass flowrate for a given power input, the mass flowrate through the fan will be determined in terms of the input power and the disk area. From equation $A-5$, the slipstream velocity $V_{3}$ can be rearranged as follows 


$$
\mathrm{V}_{3}=\left(\frac{2 \mathrm{P}_{\text {input }}}{\dot{\mathrm{m}}}\right)^{\frac{1}{2}}
$$

By substituting the slipstream velocity in A-7 into the mass flowrate in A-6

$$
\dot{\mathrm{m}}=\rho \mathrm{A}_{\text {disk }} \frac{1}{2}\left(\frac{2 \mathrm{P}_{\text {input }}}{\dot{\mathrm{m}}}\right)^{\frac{1}{2}}
$$

and simplifying, the mass flowrate is expressed in terms of the input power

$$
\dot{\mathrm{m}}=\left(\frac{1}{2} \rho \mathrm{A}_{\text {disk }}\right)^{\frac{2}{3}}\left(2 \mathrm{P}_{\text {input }}\right)^{\frac{1}{3}}
$$

Likewise, by substituting the slipstream velocity and the mass flowrate in equations A-7 and A-9 into the fan thrust in equation A-3, the static thrust produced by the fan can also be expressed solely in terms of the input power to the fan and the disk area

$$
\mathrm{T}_{\text {total }}=\left(\frac{1}{2} \rho \mathrm{A}_{\text {disk }}\right)^{\frac{1}{3}}\left(2 \mathrm{P}_{\text {input }}\right)^{\frac{2}{3}}
$$

Note that the form of the mass flowrate and the total thrust in equation A-9 and A-10 applies to both the bare and the ducted fan. Similarly, the same analysis can be done for the ducted fan.

\section{A.2. Ducted Fan}

The dimensional performance parameters of the ducted propeller from can be applied to the ducted fan by asserting that the freestream velocity is zero.

$$
\begin{gathered}
\mathrm{T}_{\text {total }}=\dot{\mathrm{m}} \mathrm{V}_{3} \\
\mathrm{~T}_{\text {fan }}=\frac{1}{2} \rho \mathrm{A}_{\text {disk }} \mathrm{V}_{3}^{2} \\
\mathrm{~T}_{\text {lip }}=\mathrm{T}_{\text {total }}-\mathrm{T}_{\text {fan }} \\
\mathrm{P}_{\text {input }}=\frac{1}{2} \dot{\mathrm{m}} \mathrm{V}_{3}^{2} \\
\dot{\mathrm{m}}=\rho \mathrm{A}_{\text {disk }} \mathrm{V}_{3}
\end{gathered}
$$


Furthermore, the lip and the fan thrust are put in terms of the power input and the disk area, as was done for the total thrust, to show that the lip thrust is equal to the fan thrust for the ducted fan. The fan thrust can be determined by substituting the slipstream velocity in equation A-7 and the mass flowrate in equation A-15 into the fan thrust in equation A-12

$$
\mathrm{T}_{\mathrm{fan}}=\frac{1}{2}\left(\rho \mathrm{A}_{\text {disk }}\right)^{\frac{1}{3}}\left(2 \mathrm{P}_{\text {input }}\right)^{\frac{2}{3}}
$$

By comparing the equations of the fan thrust and the total thrust for the ducted fan, it can be observed that the total thrust exerted on the flow is twice the thrust produced at the fan

$$
\begin{gathered}
\mathrm{T}_{\text {total }}=2\left(\frac{1}{2}\left(\rho \mathrm{A}_{\text {disk }}\right)^{\frac{1}{3}}\left(2 \mathrm{P}_{\text {input }}\right)^{\frac{2}{3}}\right) \\
\mathrm{T}_{\text {total }}=2 \mathrm{~T}_{\text {fan }}
\end{gathered}
$$

From equation A-13, the lip thrust is also the difference between the total and the fan thrust. By substituting equation A-20 into A-13, it is shown that the lip thrust is equal to the fan thrust

$$
\begin{gathered}
\mathrm{T}_{\text {lip }}=2 \mathrm{~T}_{\text {fan }}-\mathrm{T}_{\text {fan }} \\
\mathrm{T}_{\text {lip }}=\mathrm{T}_{\mathrm{fan}}
\end{gathered}
$$

The mass flowrate and the total thrust between the ducted and bare fan for two different disk areas are compared in Figure 67 and Figure 68.

\section{A.3. Results and Discussion - Incompressible Bare and Ducted Fan}

The mass flowrate and the total thrust are obtained in Figure 67 and Figure 68 for an actuator disk area of $3 \mathrm{ft}^{2}$ and $6 \mathrm{ft}^{2}$ and for the free-stream pressure and density equal to that of sea level static conditions, which are $2166.8 \mathrm{lbf} / \mathrm{ft}^{2}$ and $2.329 \times 10^{-3}$ slugs $/ \mathrm{ft}^{3}$ respectively. Note that the curves for the mass flowrate and the total thrust of the ducted fan with a disk area of $6 \mathrm{ft}^{2}$ and the bare 
fan with a disk area of $3 \mathrm{ft}^{2}$ are on top of each other in Figure 67 and Figure 68, showing that the ducted fan achieves the same mass flowrate and total static thrust as the bare fan of twice the disk area. In addition, Figure 69 gives the contribution of the lip thrust and the fan thrust to the total thrust for the case of the ducted fan. It can be observed in Figure 69 that the contribution of the lip thrust and the fan thrust are the same, as expected from the equation A-22.

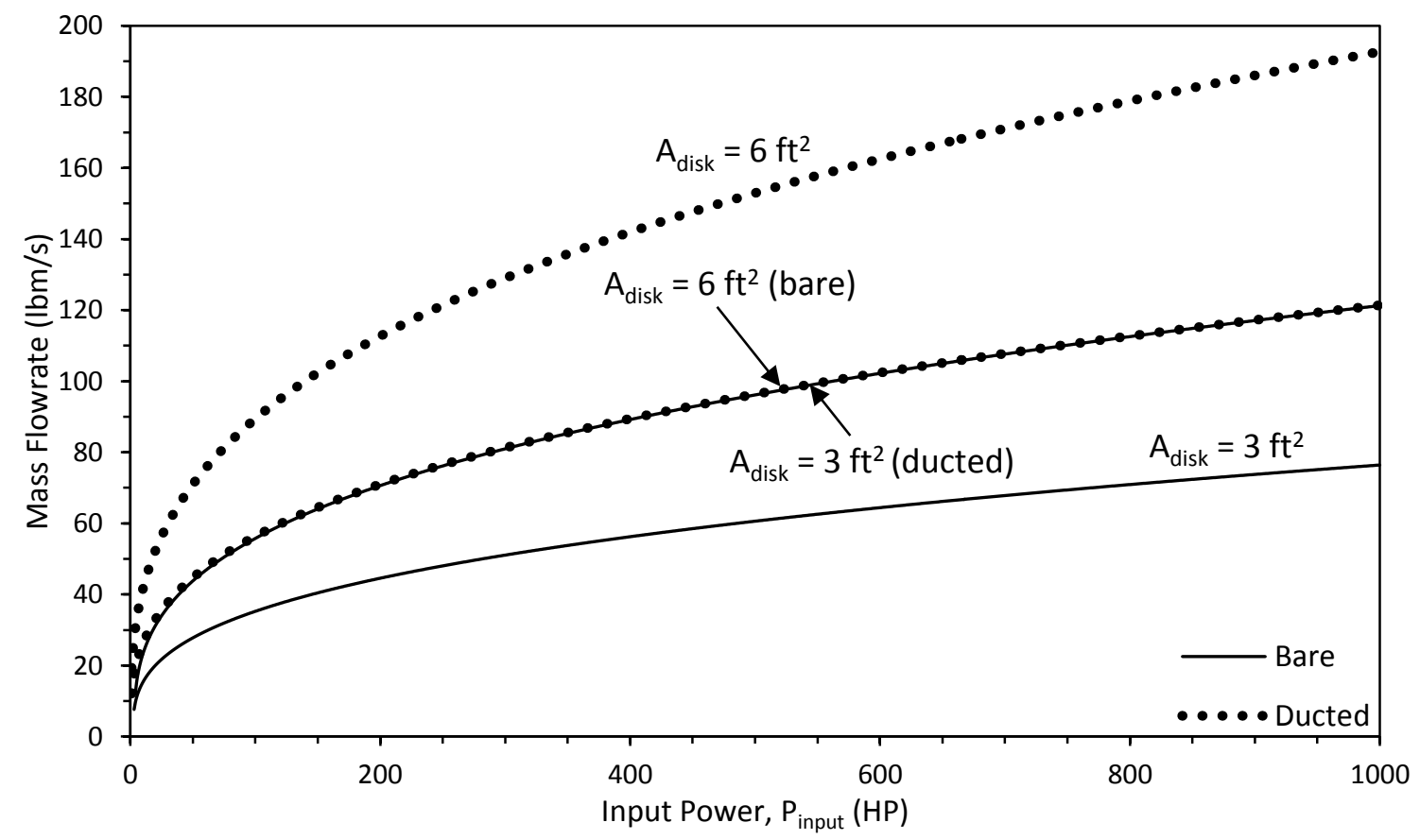

Figure 67. Comparison of mass flowrate between the incompressible ducted and bare fan for two different disk cross-sectional areas. Note that the curves for the ducted fan with a disk area of 6 $\mathrm{ft}^{2}$ and the bare fan with a disk area of $3 \mathrm{ft}^{2}$ are on top of each other. 


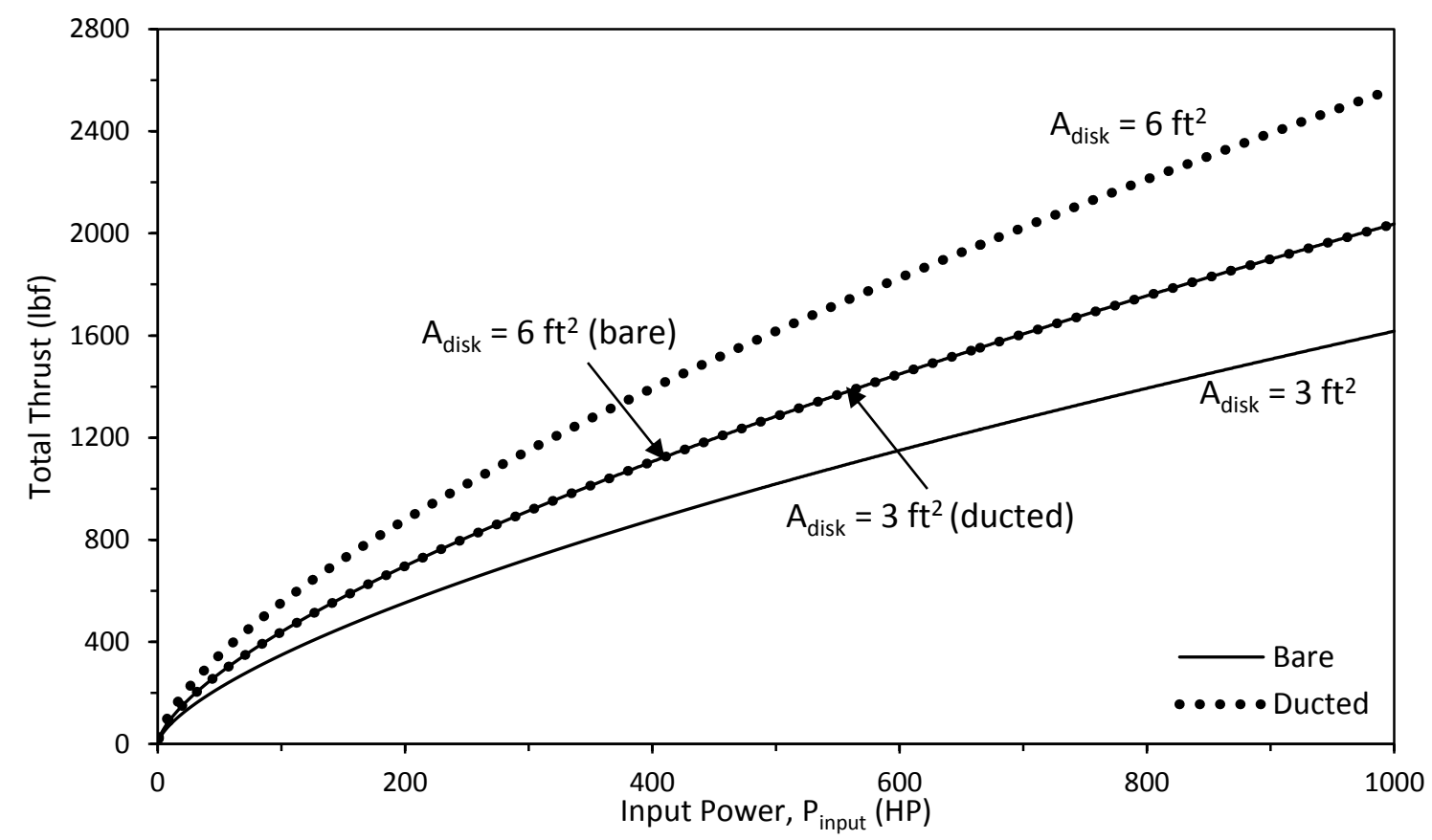

Figure 68. Comparison of the total thrust between the incompressible ducted and bare fan for two different disk cross-sectional areas. Note that the curves for the ducted fan with a disk area of 6 $\mathrm{ft}^{2}$ and the bare fan with a disk area of $3 \mathrm{ft}^{2}$ are on top of each other.

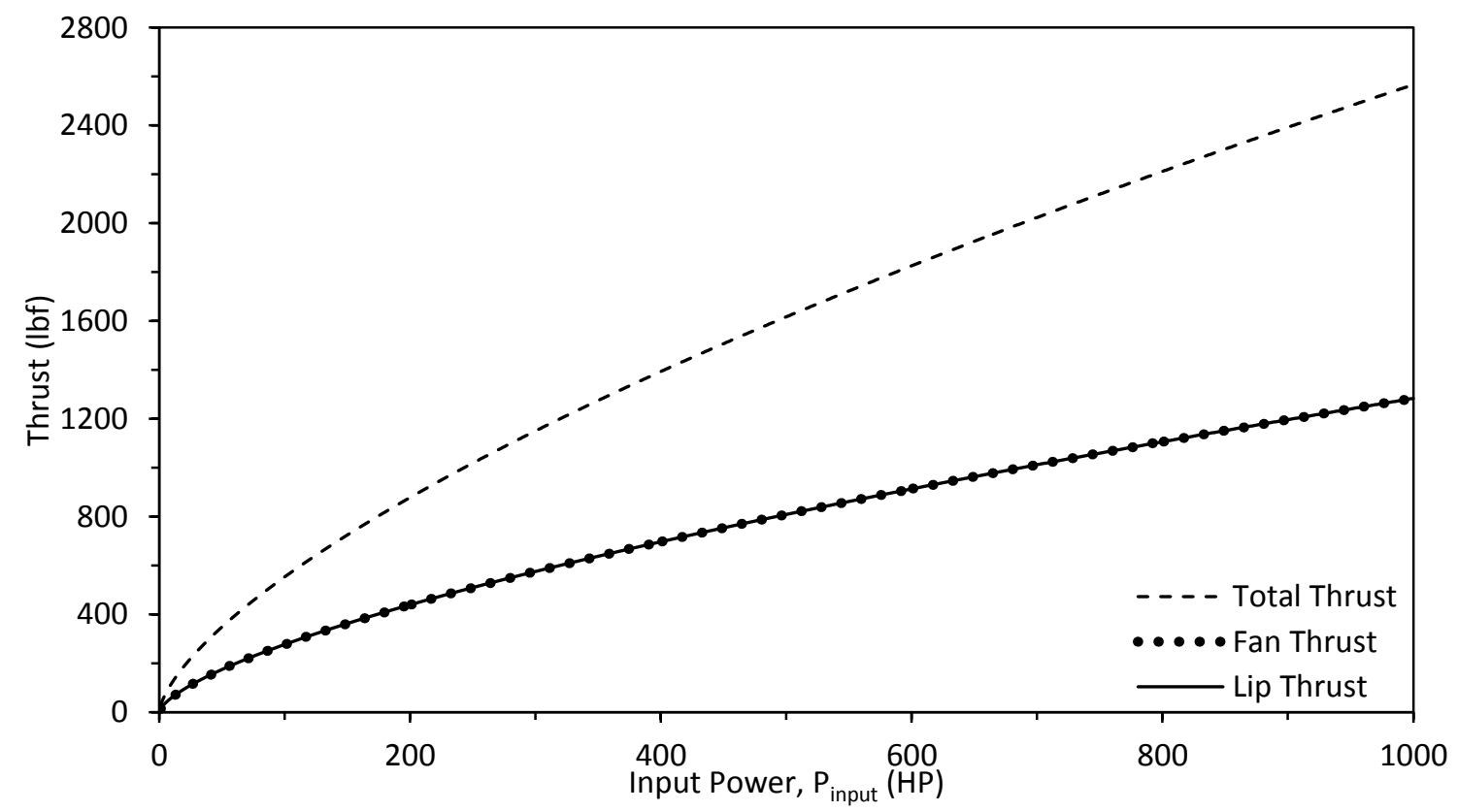

Figure 69. Comparison of the total thrust, fan thrust, and the lip thrust of the incompressible ducted fan for a disk cross-sectional area of $6 \mathrm{ft}^{2}$. Note that the curves for the fan thrust and the lip thrust are on top of each other. 


\section{B. Lip Thrust Analysis of the Compressible, Ducted Actuator Disk Theory}

To have a more complete and collective analysis of the ducted case of compressible Actuator Disk Theory, it will be shown that the lip thrust is the difference between the total thrust and the disk thrust as stated in section 2.3.1. Refer to Figure 15 for the station designations.

$$
\mathrm{T}_{\text {lip }}=\mathrm{T}_{\text {total }}-\mathrm{T}_{\text {disk }}
$$

The total thrust and the disk thrust that has previously been determined are listed below again for easy reference.

$$
\begin{gathered}
\mathrm{T}_{\text {disk }}=\dot{\mathrm{m}}\left(\mathrm{V}_{2}-\mathrm{V}_{1}\right)+\mathrm{A}_{\text {disk }}\left(\mathrm{P}_{2}-\mathrm{P}_{1}\right) \\
\mathrm{T}_{\text {total }}=\dot{\mathrm{m}}\left(\mathrm{V}_{3}-\mathrm{V}_{0}\right)
\end{gathered}
$$

These two thrust equations will be substituted into equation B-1.

$$
\mathrm{T}_{\text {lip }}=\dot{\mathrm{m}}\left(\mathrm{V}_{3}-\mathrm{V}_{0}\right)-\dot{\mathrm{m}}\left(\mathrm{V}_{2}-\mathrm{V}_{1}\right)-\mathrm{A}_{\text {disk }}\left(\mathrm{P}_{2}-\mathrm{P}_{1}\right)
$$

Using the conservation of mass, the far downstream velocity can be put in terms of the following

$$
\begin{gathered}
\rho_{0} A_{0} V_{0}=\rho_{3} A_{3} V_{3} \\
V_{3}=\frac{\rho_{0}}{\rho_{3}} \cdot \frac{A_{0}}{A_{3}} V_{0}
\end{gathered}
$$

Since the density at the far up and downstream are equal and the area at station 3 is equal to the disk area, the velocity at station 3 can be simplified.

$$
\begin{gathered}
\rho_{0}=\rho_{3} \\
A_{3}=A_{\text {disk }} \\
V_{3}=\frac{A_{0}}{A_{\text {disk }}} V_{0}
\end{gathered}
$$

From the assumption of constant duct area, a change of subscript can be applied since the velocity at station 2 and 3 are equal.

$$
\begin{gathered}
\mathrm{V}_{2}=\mathrm{V}_{3} \\
\mathrm{~V}_{2}=\frac{\mathrm{A}_{0}}{\mathrm{~A}_{\mathrm{disk}}} \mathrm{V}_{0}
\end{gathered}
$$

Therefore, by substituting equations B-10 and B-11 into the lip thrust equation in B-1 


$$
\mathrm{T}_{\text {lip }}=\dot{\mathrm{m}}\left(\frac{\mathrm{A}_{0}}{\mathrm{~A}_{\text {disk }}} \mathrm{V}_{0}-\mathrm{V}_{0}\right)-\dot{\mathrm{m}}\left(\frac{\mathrm{A}_{0}}{\mathrm{~A}_{\text {disk }}} \mathrm{V}_{0}-\mathrm{V}_{1}\right)-\mathrm{A}_{\text {disk }}\left(\mathrm{P}_{2}-\mathrm{P}_{1}\right)(
$$

The velocity at station 1 can be factored out from the first and second term of the lip thrust equation.

$$
\mathrm{T}_{\text {lip }}=\dot{\mathrm{m}} \mathrm{V}_{0}\left(\frac{\mathrm{A}_{0}}{\mathrm{~A}_{\text {disk }}}-1\right)-\dot{\mathrm{m}} \mathrm{V}_{0}\left(\frac{\mathrm{A}_{0}}{\mathrm{~A}_{\text {disk }}}-\frac{\mathrm{V}_{1}}{\mathrm{~V}_{0}}\right)-\mathrm{A}_{\text {disk }}\left(\mathrm{P}_{2}-\mathrm{P}_{1}\right)(
$$

The mass flowrate at station 0 can be substituted in.

$$
\begin{gathered}
T_{\text {lip }}=\rho_{0} V_{0}^{2} A_{0}\left(\frac{A_{0}}{A_{\text {disk }}}-1\right) \\
-\rho_{0} V_{0}^{2} A_{0}\left(\frac{A_{0}}{A_{\text {disk }}}-\frac{V_{1}}{V_{0}}\right)-A_{\text {disk }}\left(P_{2}-P_{1}\right)
\end{gathered}
$$

Due to the assumption of constant duct area, a change of subscript can be applied since the static pressure at station 2 is equal to the pressure at station 0.

$$
\begin{gathered}
P_{0}=P_{2}=P_{3} \\
T_{\text {lip }}=\rho_{0} V_{0}^{2} A_{0}\left(\frac{A_{0}}{A_{\text {disk }}}-1\right) \\
-\rho_{0} V_{0}^{2} A_{0}\left(\frac{A_{0}}{A_{\text {disk }}}-\frac{V_{1}}{V_{0}}\right)-A_{\text {disk }}\left(P_{0}-P_{1}\right)
\end{gathered}
$$

Next, the first and second term will be multiplied by the quantity $\frac{2 \mathrm{~A}_{\text {disk }}}{2 \mathrm{~A}_{\text {disk }}}$ and simplified as shown

$$
\begin{gathered}
T_{\text {lip }}=\rho_{0} V_{0}^{2} A_{0} \frac{2 A_{\text {disk }}}{2 A_{\text {disk }}}\left(\frac{A_{0}}{A_{\text {disk }}}-1\right) \\
-\rho_{0} V_{0}^{2} A_{0} \frac{2 A_{\text {disk }}}{2 A_{\text {disk }}}\left(\frac{A_{0}}{A_{\text {disk }}}-\frac{V_{1}}{V_{0}}\right)-A_{\text {disk }}\left(P_{0}-P_{1}\right) \\
T_{\text {lip }}=\frac{1}{2} \rho_{0} V_{0}^{2} A_{\text {disk }} 2 \frac{A_{0}}{A_{\text {disk }}}\left(\frac{A_{0}}{A_{\text {disk }}}-1\right) \\
-\frac{1}{2} \rho_{0} V_{0}^{2} A_{\text {disk }} 2\left(\frac{A_{0}}{A_{\text {disk }}}\right)\left(\frac{A_{0}}{A_{\text {disk }}}-\frac{V_{1}}{V_{0}}\right)-A_{\text {disk }}\left(P_{0}-P_{1}\right)
\end{gathered}
$$

Using the conservation of mass flowrate from the control volume $0-1$, a relation of the ratio $\frac{A_{0}}{A_{\text {disk }}}$ can be found.

$$
\begin{aligned}
\rho_{0} A_{0} V_{0} & =\rho_{1} A_{\text {disk }} V_{1} \\
\frac{A_{0}}{A_{\text {disk }}} & =\frac{\rho_{1}}{\rho_{0}} \frac{V_{1}}{V_{0}}
\end{aligned}
$$


Then, the area ratio is then substituted into the lip thrust equation.

$$
\begin{gathered}
\mathrm{T}_{\text {lip }}=\frac{1}{2} \rho_{0} \mathrm{~V}_{0}^{2} \mathrm{~A}_{\text {disk }} 2\left(\frac{\rho_{1}}{\rho_{0}} \frac{\mathrm{V}_{1}}{\mathrm{~V}_{0}}\right)\left(\left(\frac{\rho_{1}}{\rho_{0}} \frac{\mathrm{V}_{1}}{\mathrm{~V}_{0}}\right)-1\right) \\
-\frac{1}{2} \rho_{0} \mathrm{~V}_{0}^{2} \mathrm{~A}_{\text {disk }} 2\left(\frac{\rho_{1}}{\rho_{0}} \frac{\mathrm{V}_{1}}{\mathrm{~V}_{0}}\right)\left(\left(\frac{\rho_{1}}{\rho_{0}} \frac{\mathrm{V}_{1}}{\mathrm{~V}_{0}}\right)-\frac{\mathrm{V}_{1}}{\mathrm{~V}_{0}}\right)-\mathrm{A}_{\text {disk }}\left(\mathrm{P}_{0}-\mathrm{P}_{1}\right)
\end{gathered}
$$

Next, the third term of the lip thrust equation will be manipulated individually.

$$
\operatorname{Term}_{3}=A_{\text {disk }}\left(P_{0}-P_{1}\right)
$$

The pressure at station 0 can be factored out.

$$
\operatorname{Term}_{3}=A_{\text {disk }} P_{0}\left(1-\frac{P_{1}}{P_{0}}\right)
$$

Using the isentropic speed of sound relation, $P_{0}$ can be put in terms of $\rho_{0}, a_{0}$, and $\gamma$.

$$
\operatorname{Term}_{3}=A_{\text {disk }}\left(\frac{\rho_{0} \mathrm{a}_{0}^{2}}{\gamma}\right)\left(1-\frac{\mathrm{P}_{1}}{\mathrm{P}_{0}}\right)
$$

Next, the speed of sound at station 0 can be put in terms of the velocity to Mach number ratio.

By multiplying top and bottom by 2, the final result for the third term is the following:

$$
\begin{gathered}
\operatorname{Term}_{3}=A_{\text {disk }}\left(\frac{\rho_{0}}{\gamma} \frac{V_{0}^{2}}{M_{0}^{2}}\right)\left(1-\frac{\mathrm{P}_{1}}{\mathrm{P}_{0}}\right) \\
\operatorname{Term}_{3}=\frac{1}{2} \rho_{0} \mathrm{~V}_{0}^{2} \mathrm{~A}_{\text {disk }} \frac{2}{\gamma \mathrm{M}_{0}^{2}}\left(1-\frac{\mathrm{P}_{1}}{\mathrm{P}_{0}}\right)
\end{gathered}
$$

By substituting the manipulated term back, the lip thrust is now the following:

$$
\begin{gathered}
\mathrm{T}_{\text {lip }}=\frac{1}{2} \rho_{0} \mathrm{~V}_{0}^{2} \mathrm{~A}_{\text {disk }} 2\left(\frac{\rho_{1}}{\rho_{0}} \frac{\mathrm{V}_{1}}{\mathrm{~V}_{0}}\right)\left(\left(\frac{\rho_{1}}{\rho_{0}} \frac{\mathrm{V}_{1}}{\mathrm{~V}_{0}}\right)-1\right)-\frac{1}{2} \rho_{0} \mathrm{~V}_{0}^{2} \mathrm{~A}_{\text {disk }} 2\left(\frac{\rho_{1}}{\rho_{0}} \frac{\mathrm{V}_{1}}{\mathrm{~V}_{0}}\right)\left(\left(\frac{\rho_{1}}{\rho_{0}} \frac{\mathrm{V}_{1}}{\mathrm{~V}_{0}}\right)-\frac{\mathrm{V}_{1}}{\mathrm{~V}_{0}}\right) \\
-\frac{1}{2} \rho_{0} \mathrm{~V}_{0}^{2} \mathrm{~A}_{\text {disk }} \frac{2}{\gamma \mathrm{M}_{0}^{2}}\left(1-\frac{\mathrm{P}_{1}}{\mathrm{P}_{0}}\right)
\end{gathered}
$$

By dividing both sides by the quantity $\frac{1}{2} \rho_{0} \mathrm{~V}_{0}^{2} \mathrm{~A}_{\text {disk }}$ and simplifying the right hand side, the final lip thrust is as shown:

$$
\frac{\mathrm{T}_{\text {lip }}}{\frac{1}{2} \rho_{0} \mathrm{~V}_{0}^{2} \mathrm{~A}_{\text {disk }}}=2\left(\frac{\rho_{1}}{\rho_{0}} \frac{\mathrm{V}_{1}}{\mathrm{~V}_{0}}\right)\left(\frac{\mathrm{V}_{1}}{\mathrm{~V}_{0}}-1\right)+\frac{2}{\gamma \mathrm{M}_{0}^{2}}\left(\frac{\mathrm{P}_{1}}{\mathrm{P}_{0}}-1\right)
$$


Because the duct area at station 1 and the inlet are equivalent and the flow is isentropic, the flow conditions at station 1 are the same as the inlet flow conditions. Therefore, the subscript 1 can be replaced with i for inlet conditions.

$$
\frac{T_{\text {lip }}}{\frac{1}{2} \rho_{0} V_{0}^{2} A_{\text {disk }}}=2\left(\frac{V_{i}}{V_{0}}\right)\left(\frac{V_{i}}{V_{0}}-1\right)\left(\frac{\rho_{i}}{\rho_{0}}\right)+\frac{2}{\gamma M_{0}^{2}}\left(\frac{P_{i}}{P_{0}}-1\right)
$$

For isentropic, compressible flow, the following pressure and density ratios can be obtained from the energy equation and equation of state:

$$
\begin{aligned}
& \frac{\mathrm{P}_{\mathrm{i}}}{\mathrm{P}_{0}}=\left(1+\frac{\gamma-1}{2} \mathrm{M}_{0}^{2}\left(1-\left(\frac{\mathrm{V}_{\mathrm{i}}}{\mathrm{V}_{0}}\right)^{2}\right)\right)^{\frac{\gamma}{\gamma-1}} \\
& \frac{\rho_{\mathrm{i}}}{\rho_{0}}=\left(1+\frac{\gamma-1}{2} \mathrm{M}_{0}^{2}\left(1-\left(\frac{\mathrm{V}_{\mathrm{i}}}{\mathrm{V}_{0}}\right)^{2}\right)\right)^{\frac{1}{\gamma-1}}
\end{aligned}
$$

By substituting the above pressure and density ratios, the final lip thrust per reference force is the following.

$$
\begin{gathered}
\frac{\mathrm{T}_{\text {lip }}}{\frac{1}{2} \rho_{0} \mathrm{~V}_{0}^{2} \mathrm{~A}_{\text {disk }}}=2\left(\frac{\mathrm{V}_{\mathrm{i}}}{\mathrm{V}_{0}}\right)\left(\frac{\mathrm{V}_{\mathrm{i}}}{\mathrm{V}_{0}}-1\right)\left(1+\frac{\gamma-1}{2} \mathrm{M}_{0}^{2}\left(1-\left(\frac{\mathrm{V}_{\mathrm{i}}}{\mathrm{V}_{0}}\right)^{2}\right)\right)^{\frac{1}{\gamma-1}} \\
+\frac{2}{\gamma \mathrm{M}_{0}^{2}}\left(\left(1+\frac{\gamma-1}{2} \mathrm{M}_{0}^{2}\left(1-\left(\frac{\mathrm{V}_{\mathrm{i}}}{\mathrm{V}_{0}}\right)^{2}\right)\right)^{\frac{\gamma}{\gamma-1}}-1\right)
\end{gathered}
$$

Therefore, the resulting lip thrust from the difference between the total and disk thrust is equivalent to the lip thrust derived by Küchemann and Weber [3] in equation 2-73 by applying the conservation of momentum on the control volume 0 -i, as it should. 


\section{Compressible Bare and Ducted Fan}

This appendix will investigate the specific case of the compressible bare and ducted propeller where the freestream velocity is zero. As mentioned in Appendix A, this specific case corresponds to the applications of a fan because a fan generates a mass flow for a given power input when the freestream fluid is at rest. The performance parameters of the bare and ducted fan will be developed using the governing equations of the compressible Actuator Disk Theory given in section 2.2.1 for the bare case and section 2.3.2 for the ducted case, with the following statement

$$
\mathrm{V}_{0}=0
$$

\section{C.1. Bare Fan}

Because the bare fan is simply the specific case of the propeller where the freestream velocity is zero, the governing equations established for the bare propeller can be applied to the bare fan asserting equation $\mathrm{C}-1$. However, because $\mathrm{V}_{0}$ cannot be in the denominator of any equation, the

velocity ratio $\frac{\mathrm{V}_{1}}{\mathrm{~V}_{0}}$ in equation 3-15 has been replaced by the energy equation applied to the control volume $0-1$. In addition, the area ratio $\frac{\mathrm{A}_{3}}{\mathrm{~A}_{0}}$ in equation 3-17 has also been replaced by the conservation of mass applied to the control volume 1-3. By doing so, the governing equations of the bare fan are summarized below:

$$
\begin{gathered}
\frac{\mathrm{P}_{1}}{\mathrm{P}_{0}}=\left(\frac{1}{1+\frac{\gamma-1}{2} \mathrm{M}_{1}^{2}}\right)^{\frac{\gamma}{\gamma-1}} \\
\frac{\rho_{1}}{\rho_{0}}=\left(\frac{\mathrm{P}_{1}}{\mathrm{P}_{0}}\right)^{\frac{1}{\gamma}} \\
\frac{\gamma}{\gamma-1} \cdot \frac{\mathrm{P}_{0}}{\rho_{0}}=\frac{\mathrm{V}_{1}^{2}}{2}+\frac{\gamma}{\gamma-1} \cdot \frac{\mathrm{P}_{1}}{\rho_{1}} \\
\mathrm{M}_{3}=\mathrm{V}_{3}\left(\gamma \cdot \frac{\mathrm{P}_{0}}{\rho_{0}}\right)^{-\frac{1}{2}}
\end{gathered}
$$




$$
\begin{gathered}
\frac{\mathrm{A}_{3}}{\mathrm{~A}_{\text {disk }}}=\frac{\rho_{1}}{\rho_{3}} \cdot \frac{\mathrm{V}_{1}}{\mathrm{~V}_{3}} \\
\frac{\mathrm{A}_{3}}{\mathrm{~A}_{\mathrm{disk}}}=\frac{\mathrm{M}_{2}}{\mathrm{M}_{3}}\left(\frac{1+\frac{\gamma-1}{2} \mathrm{M}_{3}^{2}}{1+\frac{\gamma-1}{2} \mathrm{M}_{2}^{2}}\right)^{\frac{\gamma+1}{2(\gamma-1)}} \\
\frac{\mathrm{P}_{2}}{\mathrm{P}_{0}}=\left(\frac{1+\frac{\gamma-1}{2} \mathrm{M}_{3}^{2}}{1+\frac{\gamma-1}{2} \mathrm{M}_{2}^{2}}\right)^{\frac{\gamma}{\gamma-1}} \\
\frac{\rho_{2}}{\rho_{0}}=\left(\frac{\mathrm{P}_{2}}{\mathrm{P}_{0}}\right)^{\frac{1}{\gamma}} \\
\mathrm{V}_{2}=\frac{\rho_{1}}{\rho_{2}} \mathrm{~V}_{1} \\
\dot{\mathrm{m}}=\rho_{1} \mathrm{~A}_{\text {disk }} \mathrm{V}_{1} \\
\mathrm{~T}_{\text {total }}=\dot{\mathrm{m}} \mathrm{V}_{3} \\
\dot{\mathrm{m}}\left(\mathrm{V}_{2}-\mathrm{V}_{1}\right)+\mathrm{A}_{\text {disk }}\left(\mathrm{P}_{2}-\mathrm{P}_{1}\right) \\
\mathrm{T}_{\text {total }}=\mathrm{T}_{\text {fan }} \\
\mathrm{P}_{\text {input }}=\frac{1}{2} \dot{\mathrm{m}} \mathrm{V}_{3}^{2}
\end{gathered}
$$

Due to the implicit nature of the governing equations for the compressible bare fan, the mass flowrate and the total thrust cannot be expressed solely in terms of the disk area and the power input to the fan, as was done for the incompressible case. Therefore, the mass flowrate and the total thrust are solved numerically. Next, the performance parameters will be developed for the ducted fan.

\section{C.2. Ducted Fan}

Likewise, the governing equations that were established for the compressible ducted propeller can be applied to the ducted fan. Thus, the governing equations for the ducted fan are summarized as shown, including the energy equation applied to the control volume $0-1$, as was done for the bare fan, and the implications of assuming a constant-area duct:

$$
\mathrm{V}_{2}=\mathrm{V}_{3}
$$




$$
\begin{gathered}
\mathrm{P}_{0}=\mathrm{P}_{2}=\mathrm{P}_{3} \\
\frac{\mathrm{P}_{1}}{\mathrm{P}_{0}}=\left(\frac{1}{1+\frac{\gamma-1}{2} \mathrm{M}_{1}^{2}}\right)^{\frac{\gamma}{\gamma-1}} \\
\frac{\rho_{1}}{\rho_{0}}=\left(\frac{\mathrm{P}_{1}}{\mathrm{P}_{0}}\right)^{\frac{1}{\gamma}} \\
\frac{\gamma}{\gamma-1} \cdot \frac{\mathrm{P}_{0}}{\rho_{0}}=\frac{\mathrm{V}_{1}^{2}}{2}+\frac{\gamma}{\gamma-1} \cdot \frac{\mathrm{P}_{1}}{\rho_{1}} \\
\mathrm{M}_{3}=\mathrm{V}_{3}\left(\gamma \cdot \frac{\mathrm{P}_{0}}{\rho_{0}}\right)^{-\frac{1}{2}} \\
\dot{\mathrm{m}}=\rho_{1} \mathrm{~A}_{\text {disk }} \mathrm{V}_{1} \\
\mathrm{~T}_{\text {total }}=\dot{\mathrm{m}} \mathrm{V}_{3} \\
\dot{\mathrm{m}}\left(\mathrm{V}_{2}-\mathrm{V}_{1}\right)+\mathrm{A}_{\text {disk }}\left(\mathrm{P}_{2}-\mathrm{P}_{1}\right) \\
\mathrm{T}_{\text {lip }}=\mathrm{T}_{\text {total }}-\mathrm{T}_{\text {fan }} \\
\mathrm{P}_{\text {input }}=\frac{1}{2} \dot{\mathrm{m}} \mathrm{V}_{3}^{2}
\end{gathered}
$$

However, unlike the bare fan, the performance parameters, which includes the mass flowrate and the total, fan, and lip thrusts can be expressed in terms of the disk area and the power input to the fan, which will be done so next. By asserting that $\rho_{0}=\rho_{3}$ and $A_{3}=A_{\text {disk }}$ for the ducted fan, the mass flowrate can also be expressed in the following form

$$
\dot{\mathrm{m}}=\rho_{3} \mathrm{~A}_{3} \mathrm{~V}_{3}=\rho_{0} \mathrm{~A}_{\text {disk }} \mathrm{V}_{3}
$$

The power input to the ducted fan in equation C-26 can be rearranged to give the far freestream velocity in terms of the mass flowrate and the power input

$$
\mathrm{V}_{3}=\left(\frac{2 \mathrm{P}_{\text {input }}}{\dot{\mathrm{m}}}\right)^{\frac{1}{2}}
$$

By substituting the slipstream velocity found in C-28 into equation C-27, the mass flowrate is a function of the power into to the fan

$$
\dot{\mathrm{m}}=\left(\rho_{0} \mathrm{~A}_{\text {disk }}\right)^{\frac{2}{3}}\left(2 \mathrm{P}_{\text {input }}\right)^{\frac{1}{3}}
$$


Notice that the mass flowrate in equation C-29 is the same result for the incompressible, ducted fan given in Appendix A. Therefore, given any power input to the fan and the same disk area, the mass flowrate is the same for both the incompressible and compressible flow.

Likewise, by substituting the slipstream velocity in C-28 into C-23, the total thrust becomes

$$
\begin{aligned}
\mathrm{T}_{\text {total }} & =\dot{\mathrm{m}}\left(\frac{2 \mathrm{P}_{\text {input }}}{\dot{\mathrm{m}}}\right)^{\frac{1}{2}} \\
\mathrm{~T}_{\text {total }} & =\dot{\mathrm{m}}^{\frac{1}{2}}\left(2 \mathrm{P}_{\text {input }}\right)^{\frac{1}{2}}
\end{aligned}
$$

By using the mass flowrate in C-29 and simplifying, the total thrust is the same as the incompressible, ducted fan given in Appendix A.

$$
\mathrm{T}_{\text {total }}=\left(\rho \mathrm{A}_{\text {disk }}\right)^{\frac{1}{3}}\left(2 \mathrm{P}_{\text {input }}\right)^{\frac{2}{3}}
$$

Furthermore, it can be shown that the fan thrust of the incompressible ducted fan, which will be denoted as $\mathrm{T}_{\text {fan,incomp }}$, is the average of the lip and fan thrust of the compressible ducted fan. By asserting equation C-16 and C-17 into equation C-24, the fan thrust can be expressed as

$$
\mathrm{T}_{\text {fan }}=\dot{\mathrm{m}}\left(\mathrm{V}_{3}-\mathrm{V}_{1}\right)+\mathrm{A}_{\mathrm{disk}}\left(\mathrm{P}_{3}-\mathrm{P}_{1}\right)
$$

By substituting in the total thrust in C-23 and the fan thrust in C-24, the lip thrust in C-25 becomes

$$
\mathrm{T}_{\text {lip }}=\dot{\mathrm{m}} \mathrm{V}_{3}-\left(\dot{\mathrm{m}}\left(\mathrm{V}_{3}-\mathrm{V}_{1}\right)+\mathrm{A}_{\text {disk }}\left(\mathrm{P}_{3}-\mathrm{P}_{1}\right)\right)
$$

By simplifying, the lip thrust is

$$
\mathrm{T}_{\text {lip }}=\dot{\mathrm{m}} \mathrm{V}_{1}-\mathrm{A}_{\text {disk }}\left(\mathrm{P}_{3}-\mathrm{P}_{1}\right)
$$

Recall from Appendix A that the fan thrust of the incompressible ducted fan is

$$
\mathrm{T}_{\text {fan,incomp }}=\frac{1}{2}\left(\rho \mathrm{A}_{\text {disk }}\right)^{\frac{1}{3}}\left(2 \mathrm{P}_{\text {input }}\right)^{\frac{2}{3}}
$$

Now, it will be shown that the fan thrust of the incompressible ducted fan is the average of the fan and lip thrust of the compressible ducted fan

$$
\mathrm{T}_{\text {fan,incomp }}=\frac{1}{2}\left(\mathrm{~T}_{\text {fan }}+\mathrm{T}_{\text {lip }}\right)
$$


By substituting the lip thrust in C-35 and the fan thrust in C-33,

$$
\begin{aligned}
\mathrm{T}_{\text {fan,incomp }} & =\frac{1}{2}\left(\dot{\mathrm{m}}\left(\mathrm{V}_{3}-\mathrm{V}_{1}\right)+\mathrm{A}_{\text {disk }}\left(\mathrm{P}_{3}-\mathrm{P}_{1}\right)\right. \\
& \left.+\dot{\mathrm{m}} \mathrm{V}_{1}-\mathrm{A}_{\text {disk }}\left(\mathrm{P}_{3}-\mathrm{P}_{1}\right)\right)
\end{aligned}
$$

and simplifying, the incompressible fan thrust is

$$
\mathrm{T}_{\text {fan,incomp }}=\frac{1}{2} \dot{\mathrm{m}} \mathrm{V}_{3}
$$

Next by substituting the slipstream velocity in C-28 and the mass flowrate in C-29,

$$
\begin{gathered}
\mathrm{T}_{\text {fan,incomp }}=\frac{1}{2} \dot{\mathrm{m}}\left(\frac{2 \mathrm{P}_{\text {input }}}{\dot{\mathrm{m}}}\right)^{\frac{1}{2}} \\
\mathrm{~T}_{\text {fan,incomp }}=\frac{1}{2} \dot{\mathrm{m}}^{\frac{1}{2}}\left(2 \mathrm{P}_{\text {input }}\right)^{\frac{1}{2}} \\
\mathrm{~T}_{\text {fan,incomp }}=\frac{1}{2}\left(\rho_{0} \mathrm{~A}_{\text {disk }}\right)^{\frac{1}{3}}\left(2 \mathrm{P}_{\text {input }}\right)^{\frac{1}{6}}\left(2 \mathrm{P}_{\text {input }}\right)^{\frac{1}{2}}
\end{gathered}
$$

and simplifying, the incompressible fan thrust is the same as that was given in C-36. Therefore, the incompressible fan thrust is the average of the compressible fan and lip thrust. Next, the performance parameters of the fan will be compared between compressible and incompressible flow.

\section{C.3. Results and Discussion - Compressible Bare and Ducted Fan}

All solutions are determined for the free-stream pressure and density equal to that of sea level static conditions, which are $2166.8 \mathrm{lbf} / \mathrm{ft}^{2}$ and $2.329 \times 10^{-3}$ slugs $/ \mathrm{ft}^{3}$ respectively. The mass flowrate and the total thrust of the compressible fan are compared between the bare and the ducted case in Figure 70 and Figure 71 for an actuator disk area of $3 \mathrm{ft}^{2}$ and $6 \mathrm{ft}^{2}$. It can be observed that the compressible bare fan of twice the disk area performs slightly better than the ducted fan, providing slightly more mass flowrate and total thrust. This is different from the incompressible case where the bare fan of twice the disk area performs the same as the ducted fan. In addition, the mass flowrate and the total thrust of the bare fan are compared between 
incompressible and compressible flow in Figure 72 and 73, respectively, at two different disk areas of $3 \mathrm{ft}^{2}$ and $6 \mathrm{ft}^{2}$. These two figures show that the bare fan provides slightly more mass flowrate and total thrust at high input power when the flow is considered compressible. Likewise, the mass flowrate and the total thrust of the ducted fan are also compared between incompressible and compressible flow in Figure 74 and 75; however, these two performance parameters are the same for both compressible and incompressible flow for any power input to the fan at a given disk area. Lastly, Figure 76 shows that the average of the lip and fan thrust of the compressible ducted fan is equal to the lip thrust (and in turn, the fan thrust) of the incompressible case, as expected by the equation given in C-37.

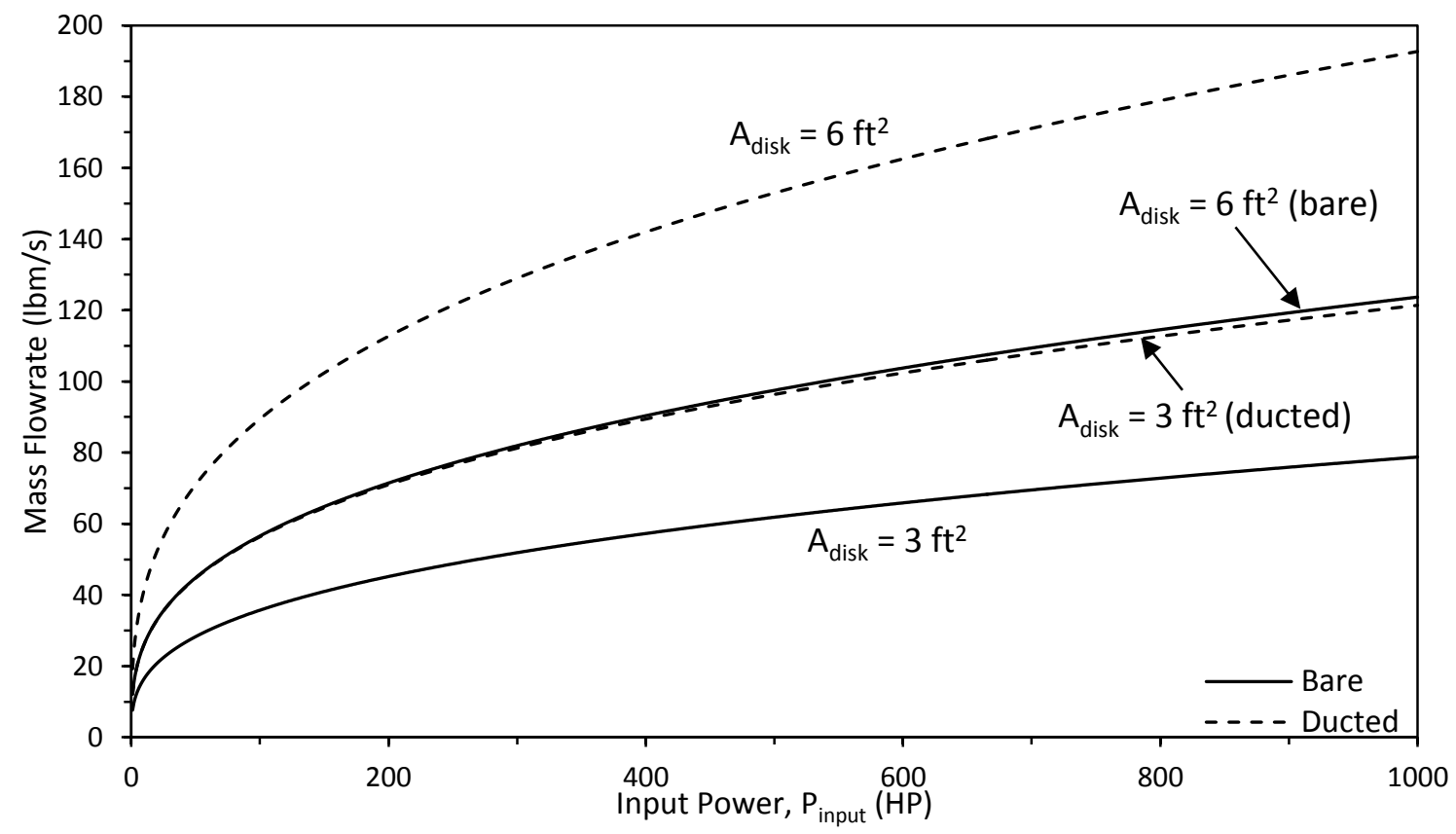

Figure 70. Comparison of the mass flowrate between the compressible ducted and bare fan at two difference disk areas. 


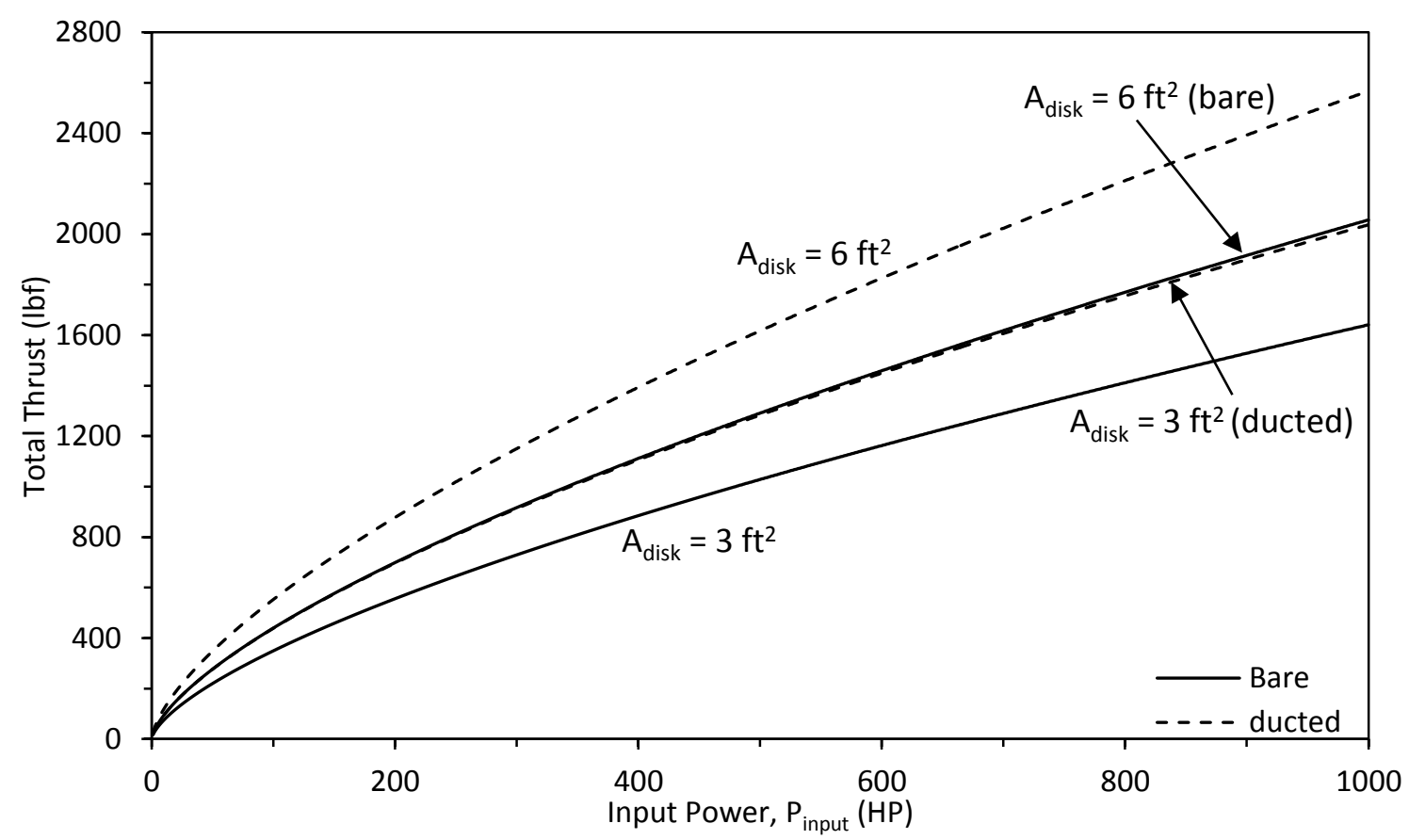

Figure 71. Comparison of the total thrust between the compressible ducted and bare fan at two different disk areas.

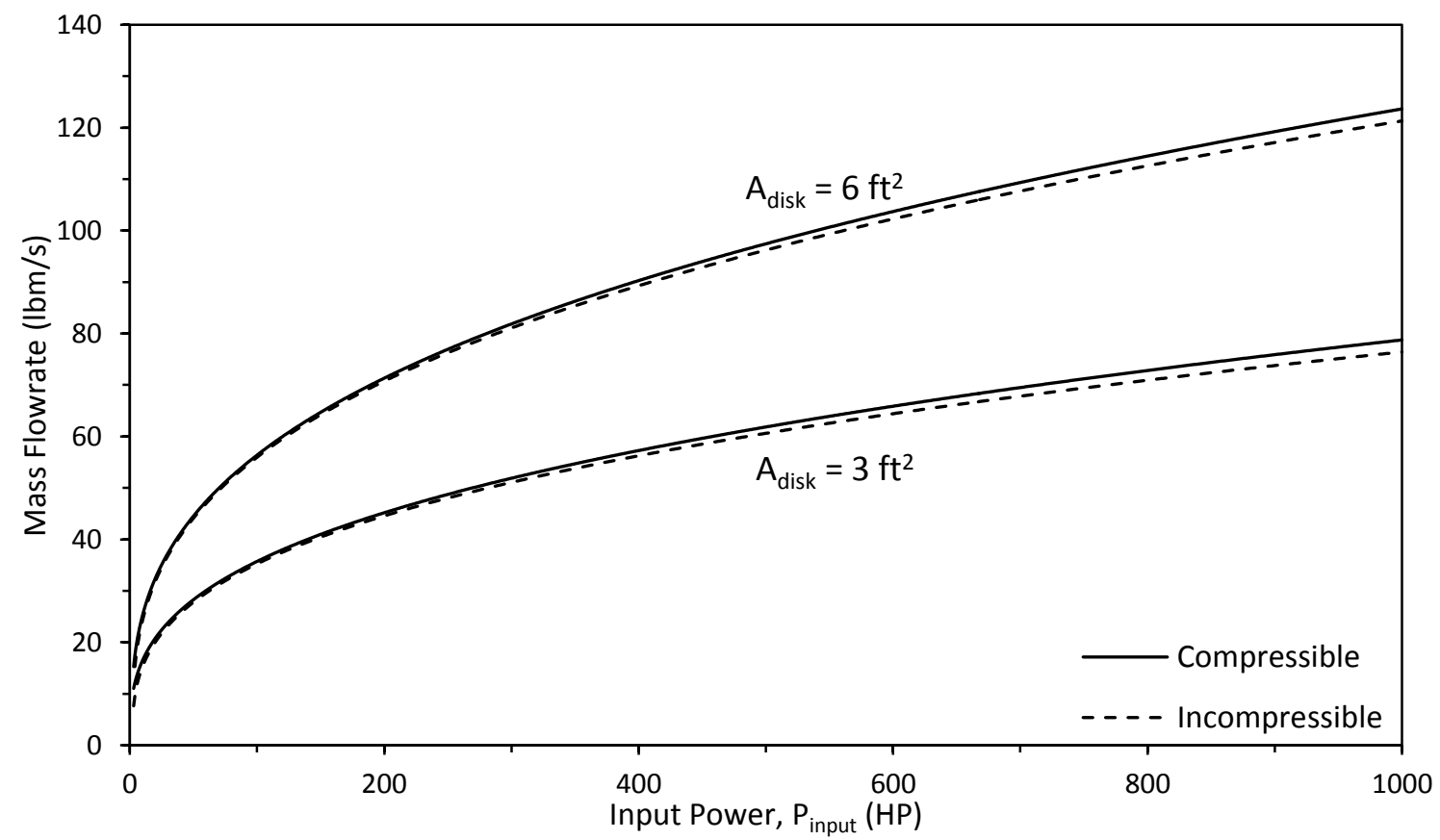

Figure 72. Comparison of the mass flowrate between the bare compressible and incompressible fan at two different disk areas. 


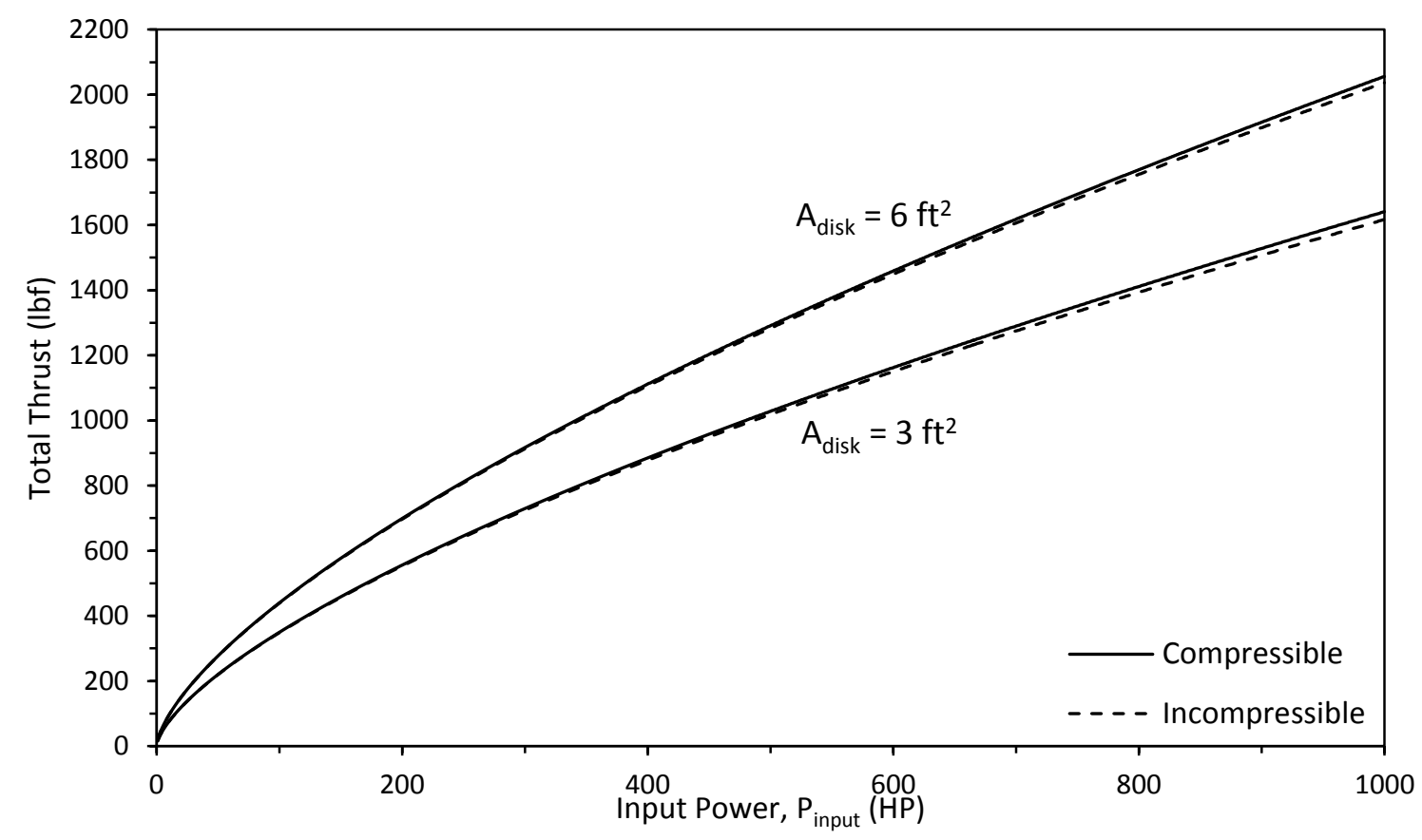

Figure 73. Comparison of the total thrust between the bare compressible and incompressible fan at two different disk areas.

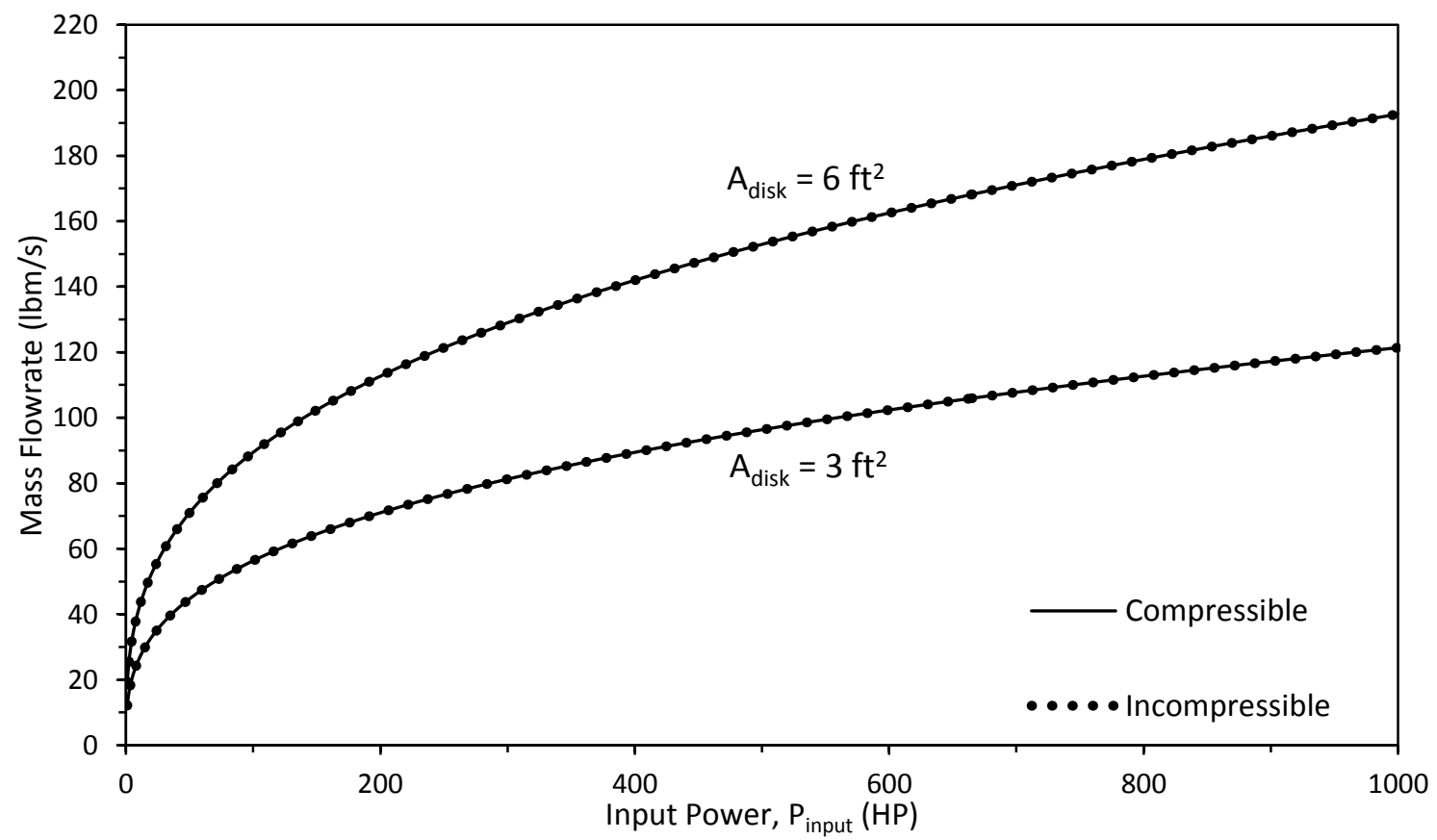

Figure 74. Comparison of the mass flowrate between the ducted incompressible and compressible fan at two different disk areas. 


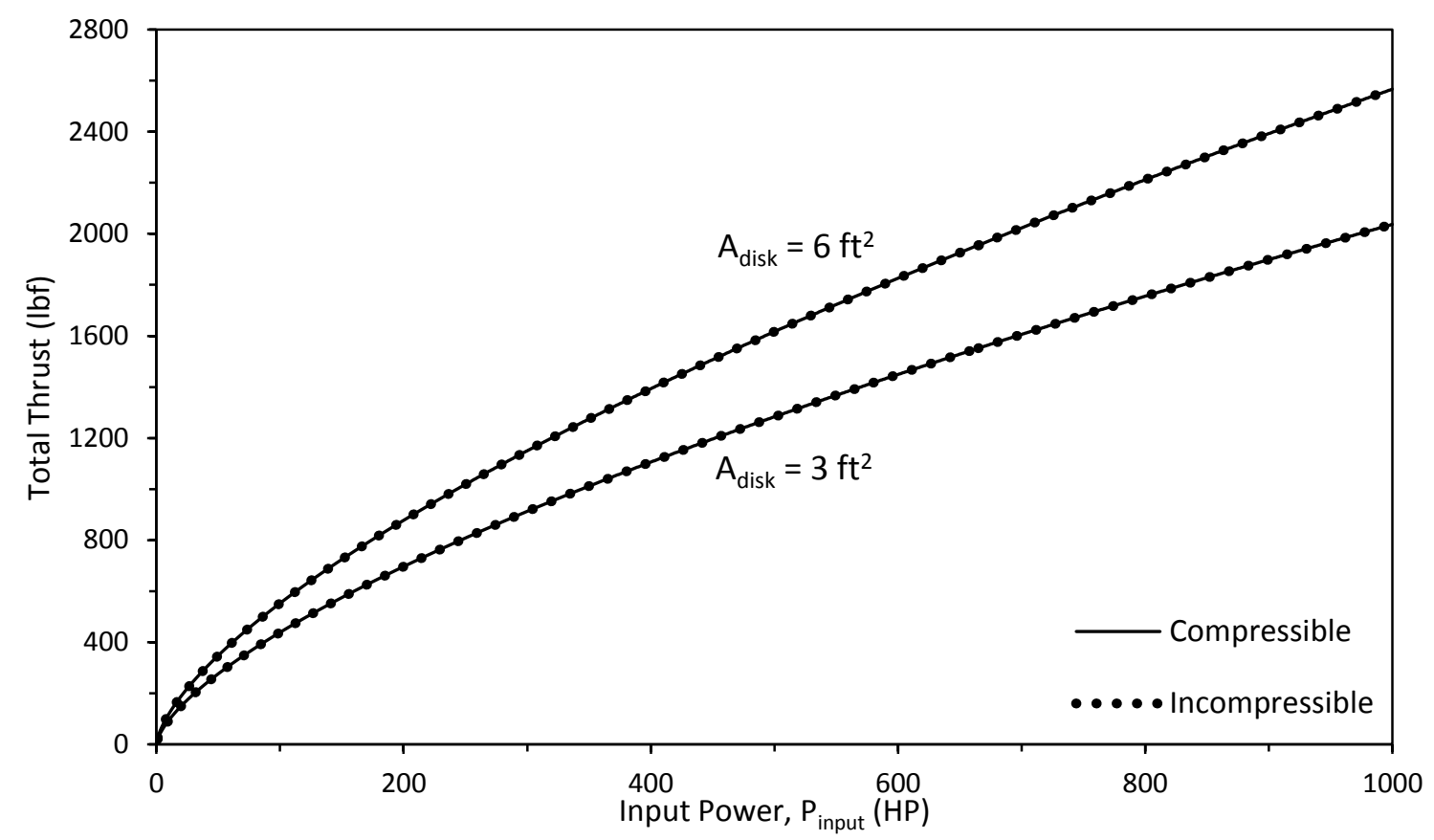

Figure 75. Comparison of the total thrust between the ducted compressible and incompressible fan at two different disk areas.

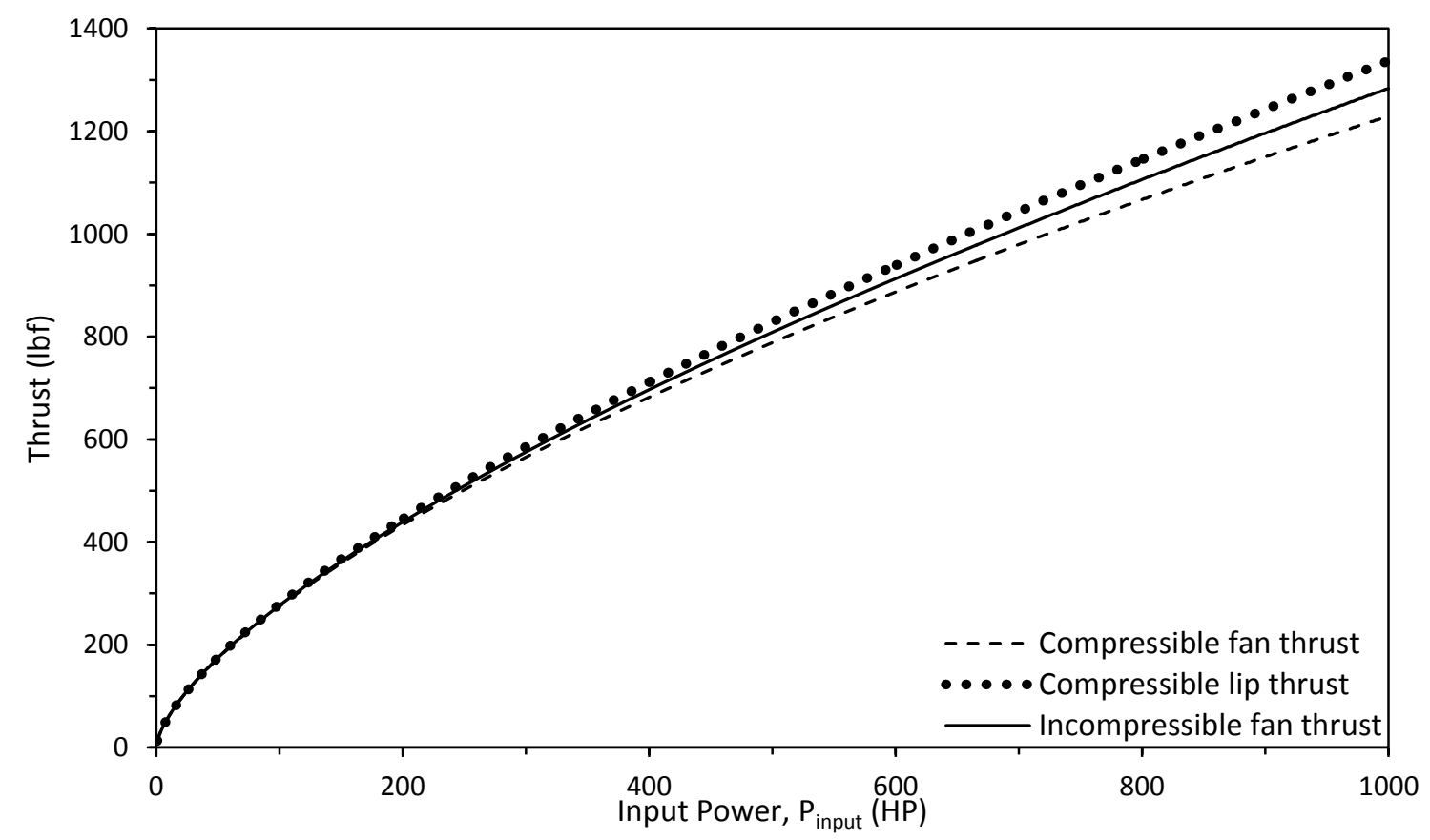

Figure 76. Comparison of the total, fan, and lip thrust between the ducted compressible and incompressible fan at a disk area of $6 \mathrm{ft}^{2}$. 


\section{EES Sheets - Compressible Bare Propeller}

File:Compressible Bare Propeller Final.EES

4/28/2017 12:53:17 PM Page 1 EES Ver. 10.096: \#0552: for use only by students and faculty, Mechanical Engineering, Dept. Cal Poly State University

NOMENCLATURE

Known: $P_{0}, \mathrm{rho}_{0}, M_{0}$

Far upstream

symbols: $V_{0}, P_{0}, M_{0}$, hoo $_{0}, A_{0}, P_{\text {to }}$

Immediately upstream of actuator disk

symbols: $V_{1}, P_{1}, M_{1}$, mo $_{1}, A_{\text {disk }}, P_{t 1}$

Immediately downstream of actuator disk

symbols: $V_{2}, P_{2}, M_{2}, \mathrm{mo}_{2}, P_{t 2}$

Far downstream

symbols: $V_{3}, P_{3}, M_{3}, m_{3}, A_{3}, P_{t 3}$

NOTES

The speed of sound is denoted by the letter ' $c$ ' instead of ' $a$ ' as defined in the thesis.

SPECIFICATIONS

Specify $P$, mo, $M$, and $A_{\text {disk }}$ of the freestream

$P_{0}=14.7 \cdot 144\left[\left[\mathrm{bf} / \mathrm{ft}^{2}\right]\right.$

po $=0.002329 \quad\left[\right.$ slug/ft $\left.{ }^{3}\right]$

$M_{0}=0.4$

$A_{d l s k}=1\left[\mathrm{ft}^{2}\right]$

$\gamma=1.4$

Specify power coefficient of the actuator disk

$C_{p}=0.1$

Note: The power coefficient is commented out when a parametric table is used.

Assumptions

$P_{0}=P_{3}$

$\rho_{0}=\rho^{3}$

Short hands

$\mathrm{k} 1=\frac{\gamma-1}{2}$ 
File:Compressible Bare Propeller Final.EES

4/28/2017 12:53:18 PM Page 2

EES Ver. 10.096: \#0552: for use only by students and faculty, Mechanical Engineering, Dept. Cal Poly State University

$\mathrm{k} 2=\frac{\gamma}{\gamma-1}$

ORDER OF EQUATION

SOLVING FOR ALL NECESSARY CONDITIONS AT 1

Far upstream conditions at 0

$c_{0}=\sqrt{\gamma \cdot \frac{P_{0}}{\rho_{0}}}$

$M_{0}=\frac{V_{0}}{c_{0}}$

Solve for $V_{0}$

Velocity ratio at 3 to 0

$\frac{V_{3}}{V_{0}}=\left[1+\frac{C_{p}}{\frac{A_{0}}{A_{d l s k}}}\right]^{\left[\begin{array}{lll}1 & / & 2\end{array}\right]}$

Solve for $\mathrm{V}_{3}$

Far downstream conditions at 3

$c_{3}=\sqrt{\gamma \cdot \frac{P_{3}}{\rho^{3}}}$

$M_{3}=\frac{V_{3}}{C_{3}}$

Solve for $\mathrm{M}_{3}$

Total Thrust

$\mathrm{T}_{\text {total }}=\dot{\mathrm{m}} \cdot\left[\mathrm{V}_{3}-\mathrm{V}_{0}\right]$

Solve for $T_{\text {total }}$

Pressure Ratio 0-1

$\frac{P_{00}}{P_{0}}=\left[1+k 1 \cdot M_{0}{ }^{2}\right]^{k 2}$

$\frac{P_{t 1}}{P_{1}}=\left[1+k 1 \cdot M_{1}{ }^{2}\right]^{12}$ 
File:Compressible Bare Propeller Final.EES

4/28/2017 12:53:18 PM Page 3

EES Ver. 10.096: \#0552: for use only by students and faculty, Mechanical Engineering, Dept. Cal Poly State University

$P_{t 0}=P_{t 1}$

Solve for $P_{1}$

Density ratio 0-1

$\frac{\rho 1}{\rho_{0}}=\left[\frac{P_{1}}{P_{0}}\right]\left[\frac{1}{\gamma}\right]$

Solve for rho,

Energy equation $0-1$

$E_{0}=\frac{V_{0}^{2}}{2}+k 2 \cdot \frac{P_{0}}{\rho_{0}}$

$E_{1}=\frac{V_{1}{ }^{2}}{2}+k 2 \cdot \frac{P_{1}}{\rho_{1}}$

$E_{0}=E_{1}$

Solve for $V_{1}$

Velocity ratio $0-1$

$V_{1}=V_{0} \cdot \frac{\rho 0}{\rho 1} \cdot \frac{A_{0}}{A_{d l s k}}$

Solve for $A_{\text {disk }}$

SOLVING FOR ALL NECESSARY CONDITIONS AT 2

Area Ratio 0-3

$\frac{A_{3}}{A_{0}}=\frac{\rho_{0}}{\rho^{3}} \cdot \frac{V_{0}}{V_{3}}$

Solve for $\mathrm{A}_{3}$

Area ratio 2-3

$\frac{A_{3}}{A_{d l 5 k}}=\frac{M_{2}}{M_{3}} \cdot\left[\left(\frac{1+k 1 \cdot M_{3}{ }^{2}}{1+k 1 \cdot M_{2}{ }^{2}}\right)^{\left(\frac{?+1}{4 \cdot k 1}\right)}\right]$

Solve for $\mathrm{M}_{2}$

Pressure ratio 2-3 
File:Compressible Bare Propeller Final.EES

4/28/2017 12:53:18 PM Page 4

EES Ver. 10.096: \#0552: for use only by students and faculty, Mechanical Engineering, Dept. Cal Poly State University

$$
\begin{aligned}
& \frac{P_{\mathrm{t} 2}}{P_{2}}=\left[1+k 1 \cdot M_{2}{ }^{2}\right]^{K 2} \\
& \frac{P_{t 3}}{P_{3}}=\left[1+k 1 \cdot M_{3}^{2}\right]^{K 2}
\end{aligned}
$$

$P_{t 2}=P_{t 3}$

Solve for $P_{2}$

Density ratio 2-3

$\frac{\rho_{2}}{\rho^{3}}=\left[\frac{P_{2}}{P_{0}}\right]\left[\frac{1}{\gamma}\right]$

Solve for $\mathrm{rhO}_{2}$

Energy equation

$E_{2}=\frac{V_{2}{ }^{2}}{2}+k 2 \cdot \frac{P_{2}}{\rho^{2}}$

$E_{3}=\frac{V_{3}{ }^{2}}{2}+k 2 \cdot \frac{P_{3}}{\rho^{3}}$

$E_{2}=E_{3}$

Solve for $\mathrm{V}_{2}$

Thrust equation at disk

NEED $V_{1}, V_{2}, P_{1}, P_{2}$

$T_{\text {prop }}=\dot{m} \cdot\left[V_{2}-V_{1}\right]+A_{\text {dlsk }} \cdot\left[P_{2}-P_{1}\right]$

$T_{\text {total }}=T_{\text {prop }}$

Efficiency and Coefficients

Power available within free-flowing wind in area equal to rotor area $A_{\text {disk }}$

$P_{\text {avallable }}=\rho_{0} \cdot V_{0}{ }^{3} \cdot \frac{A_{\text {dlsk }}}{2}$

Power coefficient

$C_{P}=\frac{P_{\text {input }}}{P_{\text {avallable }}}$ 
File:Compressible Bare Propeller Final.EES

4/28/2017 12:53:18 PM Page 5

EES Ver. 10.096: \#0552: for use only by students and faculty, Mechanical Engineering, Dept. Cal Poly State University

Total Thrust Coefficient

$C_{T}=\frac{T_{\text {total }}}{\rho_{0} \cdot V_{0}{ }^{2} \cdot \frac{A_{d l s k}}{2}}$

Efficiency

$\eta=\frac{C_{T}}{C_{p}}$

Mass Flowrate

$\dot{\mathrm{m}}=\mathrm{V}_{1} \cdot \rho_{1} \cdot A_{\mathrm{dlsk}}$

NORMALIZATIONS

Ratios

$V_{10}=\frac{V_{1}}{V_{0}}$

$V_{20}=\frac{V_{2}}{V_{0}}$

$V_{30}=\frac{V_{3}}{V_{0}}$

$M_{10}=\frac{M_{1}}{M_{0}}$

$M_{20}=\frac{M_{2}}{M_{0}}$

$M_{30}=\frac{M_{3}}{M_{0}}$

$P_{10}=\frac{P_{1}}{P_{0}}$

$P_{20}=\frac{P_{2}}{P_{0}}$

$\rho_{10}=\frac{\rho_{1}}{\rho_{0}}$

$\rho_{20}=\frac{\rho^{2}}{\rho 0}$ 
File:Compressible Bare Propeller Final.EES

4/28/2017 12:53:18 PM Page 6 EES Ver. 10.096: \#0552: for use only by students and faculty, Mechanical Engineering, Dept. Cal Poly State University

$A_{01}=\frac{A_{0}}{A_{d l s k}}$
$A_{31}=\frac{A_{3}}{A_{d l s k}}$

Pressure-rise Coefficient

$C_{6, P}=\frac{P_{2}-P_{1}}{0.5 \cdot \rho_{0} \cdot V_{0}^{2}}$

Dimensionless mass flowrate

$\dot{\mathrm{m}}_{d}=\frac{\dot{\mathrm{m}}}{\rho_{0} \cdot V_{0} \cdot A_{d l s k}}$ 


\section{E. EES Sheets - Compressible Ducted Propeller}

File:Compressible Ducted Propeller Final.EES

4/28/2017 12:57:58 PM Page 1

EES Ver. 10.096: \#0552: for use only by students and faculty, Mechanical Engineering, Dept. Cal Poly State University

NOMENCLATURE

Known: $P_{0}$, rho $_{0}, M_{0}$

Far upstream

symbols: $V_{0}, P_{0}, M_{0}$, mo $_{0}, A_{0}, P_{\text {to }}$

Immediately upstream of actuator disk

symbols: $V_{1}, P_{1}, M_{1}$, mo $_{1}, A_{\text {disk }}, P_{t 1}$

Immediately downstream of actuator disk

symbols: $V_{2}, P_{2}, M_{2}, \mathrm{mo}_{2}, P_{t 2}$

Far downstream

symbols: $V_{3}, P_{3}, M_{3}, \mathrm{mo}_{3}, A_{3}, P_{t 3}$

NOTES

The speed of sound is denoted by the letter ' $c$ ' instead of ' $a$ ' as defined in the thesis.

SPECIFICATIONS

Specify $P_{\text {input }}, r h o, M$, and $A_{\text {disk }}$ of the freestream

$M_{0}=0.4$

$P_{0}=14.7 \cdot 144 \quad\left[\mathrm{lbf} / \mathrm{ft}^{2}\right]$

$\rho^{0}=0.002329 \quad\left[\right.$ slug/ $\left./ \mathrm{ft}^{3}\right]$

Adlsk $=1\left[\mathrm{ft}^{2}\right]$

$\gamma=1.4$

Specify power coefficient at actuator disk

$C_{P}=0.1$

Note: The power coefficient is commented out when a parametric table is used.

Assumptions

$P_{0}=P_{3}$

$\rho^{0}=\rho^{3}$

$A_{3}=A_{d l s k}$

Short hands 
File:Compressible Ducted Propeller Final.EES

4/28/2017 12:57:58 PM Page 2

EES Ver. 10.096: \#0552: for use only by students and faculty, Mechanical Engineering, Dept. Cal Poly State University

$\mathrm{k} 1=\frac{\gamma-1}{2}$

$k 2=\frac{\gamma}{\gamma-1}$

ORDER OF EQUATION

SOLVING FOR ALL NECESSARY CONDITIONS AT 1

Far upstream conditions at 0

$c_{0}=\sqrt{\gamma \cdot \frac{P_{0}}{\rho_{0}}}$

$M_{0}=\frac{V_{0}}{c_{0}}$

Solve for $V_{0}$

Velocity ratio at 0-3

$\frac{V_{3}}{V_{0}}=\left[1+\frac{C_{p}}{\frac{A_{0}}{A_{d l s k}}}\right]^{\left[\begin{array}{lll}1 & 1 & 2\end{array}\right]}$

Solve for $V_{3}$

Far downstream conditions at 3

$c_{3}=\sqrt{7 \cdot \frac{P_{3}}{\rho_{3}}}$

$M_{3}=\frac{V_{3}}{c_{3}}$

Solve for $\mathrm{M}_{3}$

Total Thrust

$\mathrm{T}_{\text {total }}=\dot{\mathrm{m}} \cdot\left[\mathrm{V}_{3}-\mathrm{V}_{0}\right]$

Solve for $T_{\text {total }}$

Pressure Ratio 0-1

$\frac{P_{00}}{P_{0}}=\left[1+k 1 \cdot M_{0}^{2}\right]^{k 2}$ 
File:Compressible Ducted Propeller Final.EES

4/28/2017 12:57:58 PM Page 3

EES Ver. 10.096: \#0552: for use only by students and faculty, Mechanical Engineering, Dept. Cal Poly State University

$$
\begin{aligned}
& \frac{P_{t 1}}{P_{1}}=\left[1+k 1 \cdot M_{1}^{2}\right]^{K 2} \\
& P_{t 0}=P_{t 1}
\end{aligned}
$$

Solve for $P_{1}$

Density ratio 0-1

$\frac{\rho_{1}}{\rho_{0}}=\left[\frac{P_{1}}{P_{0}}\right]\left[\frac{1}{\gamma}\right]$

Solve for rho,

Energy equation 0-1

$E_{0}=\frac{V_{0}^{2}}{2}+k 2 \cdot \frac{P_{0}}{\rho_{0}}$

$E_{1}=\frac{V_{1}{ }^{2}}{2}+k 2 \cdot \frac{P_{1}}{\rho_{1}}$

$E_{0}=E_{1}$

Solve for $V_{1}$

Velocity ratio 0-1

$\frac{V_{1}}{V_{0}}=\frac{\rho_{0}}{\rho_{1}} \cdot \frac{A_{0}}{A_{d l s k}}$

Solve for $A_{\text {disk }}$

SOLVING FOR ALL NECESSARY CONDITIONS AT 2

Area Ratio 0-3

$\frac{A_{3}}{A_{0}}=\frac{\rho_{0}}{\rho^{3}} \cdot \frac{V_{0}}{V_{3}}$

Solve for $A_{3}$

Area ratio 2-3

$\frac{A_{3}}{A_{d i s k}}=\frac{M_{2}}{M_{3}} \cdot\left[\left(\frac{1+k 1 \cdot M_{3}^{2}}{1+k 1 \cdot M_{2}{ }^{2}}\right)^{\left(\frac{?+1}{4 \cdot k 1}\right)}\right]$

Solve for $\mathrm{M}_{2}$ 
File:Compressible Ducted Propeller Final.EES

4/28/2017 12:57:58 PM Page 4

EES Ver. 10.096: \#0552: for use only by students and faculty, Mechanical Engineering, Dept. Cal Poly State University

Pressure ratio 2-3

$\frac{P_{t 2}}{P_{2}}=\left[1+k 1 \cdot M_{2}^{2}\right]^{k 2}$

$\frac{P_{t 3}}{P_{3}}=\left[1+k 1 \cdot M_{3}^{2}\right]^{k 2}$

$P_{t 2}=P_{t 3}$

Solve for $P_{2}$

Density ratio 2-3

$\frac{\rho^{2}}{\rho^{3}}=\left[\frac{P_{2}}{P_{0}}\right]\left[\frac{1}{\gamma}\right]$

Solve for $\mathrm{rhO}_{2}$

Energy equation

$E_{2}=\frac{V_{2}{ }^{2}}{2}+k 2 \cdot \frac{P_{2}}{\rho^{2}}$

$E_{3}=\frac{V_{3}^{2}}{2}+k 2 \cdot \frac{P_{3}}{\rho^{3}}$

$E_{2}=E_{3}$

Solve for $\mathrm{V}_{2}$

Thrust equations

NEED $V_{1}, V_{2}, P_{1}, P_{2}$

$T_{\text {prop }}=\dot{m} \cdot\left[V_{2}-V_{1}\right]+A_{\text {dlsk }} \cdot\left[P_{2}-P_{1}\right]$

$T_{\text {IIp }}=T_{\text {total }}-T_{\text {prop }}$

Efficiency and Coefficients

Power available within free-flowing wind in area equal to rotor area $A_{\text {disk }}$

Pavallable $=\rho_{0} \cdot V_{0}{ }^{3} \cdot \frac{\text { Adlisk }}{2}$

Power coefficient 
File:Compressible Ducted Propeller Final.EES

4/28/2017 12:57:59 PM Page 5

EES Ver. 10.096: \#0552: for use only by students and faculty, Mechanical Engineering, Dept. Cal Poly State University

$C_{P}=\frac{P_{\text {Input }}}{P_{\text {avallable }}}$

Total Thrust Coefficient

$C_{T}=\frac{T_{\text {total }}}{\rho_{0} \cdot V_{0}{ }^{2} \cdot \frac{A_{d l s k}}{2}}$

Efficiency

$\eta=\frac{C_{T}}{C_{p}}$

Mass Flowrate

$\dot{\mathrm{m}}=\mathrm{V}_{1} \cdot \rho_{1} \cdot \mathrm{A}_{\mathrm{dlsk}}$

NORMALIZATIONS

Ratios

$V_{10}=\frac{V_{1}}{V_{0}}$

$V_{20}=\frac{V_{2}}{V_{0}}$

$V_{30}=\frac{V_{3}}{V_{0}}$

$M_{10}=\frac{M_{1}}{M_{0}}$

$M_{20}=\frac{M_{2}}{M_{0}}$

$M_{30}=\frac{M_{3}}{M_{0}}$

$P_{10}=\frac{P_{1}}{P_{0}}$

$P_{20}=\frac{P_{2}}{P_{0}}$

$\rho_{10}=\frac{\rho 1}{\rho 0}$ 
File:Compressible Ducted Propeller Final.EES

4/28/2017 12:57:59 PM Page 6

EES Ver. 10.096: \#0552: for use only by students and faculty, Mechanical Engineering, Dept. Cal Poly State University

$\rho^{20}=\frac{\rho^{2}}{\rho^{0}}$

$A_{01}=\frac{A_{0}}{A_{d l s k}}$

$A_{31}=\frac{A_{3}}{A_{d l s k}}$

Pressure-rise Coefficient

$C_{6, P}=\frac{P_{2}-P_{1}}{0.5 \cdot \rho_{0} \cdot V_{0}{ }^{2}}$

Dimensionless mass flowrate

$\dot{m}_{d}=\frac{\dot{m}}{\rho_{0} \cdot V_{0} \cdot A_{d l s k}}$

Lip Thrust Coefficient

$C_{T, \text { Il }}=\frac{T_{\text {ilp }}}{\rho_{0} \cdot V_{0}{ }^{2} \cdot \frac{A_{d i s k}}{2}}$

Propeller Thrust Coefficient

$C_{T, \text { prop }}=\frac{T_{\text {prop }}}{\rho_{0} \cdot V_{0}{ }^{2} \cdot \frac{A_{d l s k}}{2}}$ 
F. $\quad$ EES Sheets - Compressible Bare Turbine

File:Compressible Bare Turbine Final.EES

4/28/2017 1:50:26 PM Page 1

EES Ver. 10.096: \#0552: for use only by students and faculty, Mechanical Engineering, Dept. Cal Poly State University

NOMENCLATURE

Known: $P_{0}, r h o_{O_{1}} M_{0}$

Far upstream

symbols: $V_{0}, P_{0}, M_{0}, m_{0}, A_{0}, P_{t 0}$

Immediately upstream of actuator disk

symbols: $V_{1}, P_{1}, M_{1}$, mo $1, A_{\text {disk }}, P_{t 1}$

Immediately downstream of actuator disk

symbols: $V_{2}, P_{2}, M_{2}, \mathrm{mo}_{2}, P_{t 2}$

Far downstream

symbols: $V_{3}, P_{3}, M_{3}, \mathrm{mo}_{3}, A_{3}, P_{t 3}$

NOTES

The speed of sound is denoted by the letter ' $c$ ' instead of 'a' as defined in the thesis.

SPECIFICATIONS

Specify $P_{\text {extracted, }}$ mo, $M$, and $A_{\text {disk }}$ of the freestream

$M_{0}=0.4$

$P_{0}=14.7 \cdot 144\left[\mathrm{lbf} / \mathrm{ft}^{2}\right]$

po $=0.002329 \quad\left[\right.$ slug/ $\left./ \mathrm{ft}^{3}\right]$

Adlak $=1\left[\mathrm{ft}^{2}\right]$

$\gamma=1.4$

Specify velocity ratio $r$

$r=\frac{V_{3}}{V_{0}}$

$r=0.85$

Note: The velocity ratio $r$ is commented out when a parametric table is used.

Assumptions

$P_{0}=P_{3}$

$\rho^{0}=\rho^{3}$

Short hands 
File:Compressible Bare Turbine Final.EES

4/28/2017 1:50:27 PM Page 2

EES Ver. 10.096: \#0552: for use only by students and faculty, Mechanical Engineering, Dept. Cal Poly State University

$\mathrm{k} 1=\frac{\gamma-1}{2}$

$\mathrm{k} 2=\frac{\gamma}{\gamma-1}$

ORDER OF EQUATION

SOLVING FOR ALL NECESSARY CONDITIONS AT 1

Far upstream conditions at 0

$c_{0}=\sqrt{\gamma \cdot \frac{P_{0}}{\rho_{0}}}$

$M_{0}=\frac{V_{0}}{c_{0}}$

Solve for $V_{0}$

Far downstream conditions at 3

$c_{3}=\sqrt{\gamma \cdot \frac{P_{3}}{\rho^{3}}}$

$M_{3}=\frac{V_{3}}{c_{3}}$

Solve for $V_{3}$

Total Thrust

$D_{\text {total }}=\dot{m} \cdot\left[V_{0}-V_{3}\right]$

Solve for $D_{\text {total }}$

Pressure Ratio 0-1

$\frac{P_{00}}{P_{0}}=\left[1+k 1 \cdot \mathrm{Mo}^{2}\right]^{k 2}$

$\frac{P_{t 1}}{P_{1}}=\left[1+k 1 \cdot M_{1}^{2}\right]^{k 2}$

$P_{10}=P_{t 1}$

Solve for $P_{1}$

Density ratio 0-1 
File:Compressible Bare Turbine Final.EES

4/28/2017 1:50:27 PM Page 3

EES Ver. 10.096: \#0552: for use only by students and faculty, Mechanical Engineering, Dept. Cal Poly State University

$\frac{\rho_{1}}{\rho_{0}}=\left[\frac{P_{1}}{P_{0}}\right]\left[\frac{1}{\gamma}\right]$

Solve for rho,

Energy equation $0-1$

$E_{0}=\frac{V_{0}^{2}}{2}+k 2 \cdot \frac{P_{0}}{\rho_{0}}$

$E_{1}=\frac{V_{1}{ }^{2}}{2}+k 2 \cdot \frac{P_{1}}{\rho 1}$

$E_{0}=E_{1}$

Solve for $V_{1}$

Velocity ratio 0-1

$\frac{V_{1}}{V_{0}}=\frac{\rho_{0}}{\rho_{1}} \cdot \frac{A_{0}}{A_{\text {dlsk }}}$

Solve for $A_{\text {disk }}$

SOLVING FOR ALL NECESSARY CONDITIONS AT 2

Area Ratio 0-3

$\frac{A_{3}}{A_{0}}=\frac{\rho_{0}}{\rho^{3}} \cdot \frac{V_{0}}{V_{3}}$

Solve for $\mathrm{A}_{3}$

Area ratio 2-3

$\frac{A_{3}}{A_{d 15 k}}=\frac{M_{2}}{M_{3}} \cdot\left[\left(\frac{1+k 1 \cdot M_{3}{ }^{2}}{1+k 1 \cdot M_{2}{ }^{2}}\right)^{\left(\frac{?+1}{4 \cdot k 1}\right)}\right]$

Solve for $\mathrm{M}_{2}$

Pressure ratio 2-3

$\frac{P_{t 2}}{P_{2}}=\left[1+k_{1} \cdot M_{2}{ }^{2}\right]^{K 2}$ 
File:Compressible Bare Turbine Final.EES

4/28/2017 1:50:27 PM Page 4

EES Ver. 10.096: \#0552: for use only by students and faculty, Mechanical Engineering, Dept. Cal Poly State University

$$
\begin{aligned}
& \frac{P_{t 3}}{P_{3}}=\left[1+k 1 \cdot M_{3}^{2}\right]^{k 2} \\
& P_{t 2}=P_{t 3}
\end{aligned}
$$

Solve for $P_{2}$

Density ratio 2-3

$\frac{\rho^{2}}{\rho^{3}}=\left[\frac{P_{2}}{P_{0}}\right]\left[\frac{1}{\gamma}\right]$

Solve for $\mathrm{rhO}_{2}$

Energy equation

$E_{2}=\frac{V_{2}{ }^{2}}{2}+k 2 \cdot \frac{P_{2}}{\rho^{2}}$

$E_{3}=\frac{V_{3}{ }^{2}}{2}+k 2 \cdot \frac{P_{3}}{\rho^{3}}$

$E_{2}=E_{3}$

Solve for $\mathrm{V}_{2}$

Thrust equation at disk

NEED $V_{1}, V_{2}, P_{1}, P_{2}$

$D_{\text {turo }}=\dot{m} \cdot\left[V_{1}-V_{2}\right]+A_{d l s k} \cdot\left[P_{1}-P_{2}\right]$

$D_{\text {total }}=$ Dturo

Power equations

$\frac{P_{\text {extracted }}}{\dot{m}}=\frac{V_{0}{ }^{2}-V_{3}{ }^{2}}{2}$

Power available within free-flowing wind in area equal to rotor area $A_{\text {disk }}$

Pavallable $=\rho_{0} \cdot V_{0}{ }^{3} \cdot \frac{\text { Adlsk }}{2}$

Power Extraction Efficiency

$\eta=\frac{P_{\text {extracted }}}{P_{\text {avallable }}}$ 
File:Compressible Bare Turbine Final.EES

4/28/2017 1:50:27 PM Page 5

EES Ver. 10.096: \#0552: for use only by students and faculty, Mechanical Engineering, Dept. Cal Poly State University

Total Thrust Coefficient

$C_{0}=\frac{D_{\text {total }}}{\rho_{0} \cdot V_{0}{ }^{2} \cdot \frac{A_{\text {dlsk }}}{2}}$

Mass Flowrate

$\dot{\mathrm{m}}=\mathrm{V}_{1} \cdot \rho_{1} \cdot \mathrm{A}_{\mathrm{dlsk}}$

NORMALIZATIONS

Ratios

$V_{10}=\frac{V_{1}}{V_{0}}$

$V_{20}=\frac{V_{2}}{V_{0}}$

$V_{30}=\frac{V_{3}}{V_{0}}$

$M_{10}=\frac{M_{1}}{M_{0}}$

$M_{20}=\frac{M_{2}}{M_{0}}$

$M_{30}=\frac{M_{3}}{M_{0}}$

$P_{10}=\frac{P_{1}}{P_{0}}$

$P_{20}=\frac{P_{2}}{P_{0}}$

$\rho_{10}=\frac{\rho 1}{\rho 0}$

$\rho^{20}=\frac{\rho 2}{\rho 0}$

$A_{01}=\frac{A_{0}}{A_{\text {dlsk }}}$ 
File:Compressible Bare Turbine Final.EES

4/28/2017 1:50:27 PM Page 6

EES Ver. 10.096: \#0552: for use only by students and faculty, Mechanical Engineering, Dept. Cal Poly State University

$A_{31}=\frac{A_{3}}{A_{d 1 s k}}$

Pressure-rise Coefficient

$C_{6, P}=\frac{P_{2}-P_{1}}{0.5 \cdot \rho_{0} \cdot V_{0}^{2}}$

Dimensionless mass flowrate

$\dot{\mathrm{m}}_{d}=\frac{\dot{\mathrm{m}}}{\rho_{0} \cdot \mathrm{V}_{0} \cdot A_{\text {dlisk }}}$ 


\section{G. EES Sheets - Compressible Ducted Turbine}

File:Compressible Ducted Turbine Final.EES

4/28/2017 1:45:44 PM Page 1

EES Ver. 10.096: \#0552: for use only by students and faculty, Mechanical Engineering, Dept. Cal Poly State University

NOMENCLATURE

Known: $P_{0}$, rho $_{0}, M_{0}$

Far upstream

symbols: $V_{0}, P_{0}, M_{0}$, mo $_{0}, A_{0}, P_{t o}$

Immediately upstream of actuator disk

symbols: $V_{1}, P_{1}, M_{1}$, mo $1, A_{\text {disk }}, P_{t 1}$

Immediately downstream of actuator disk

symbols: $V_{2}, P_{2}, M_{2}, \mathrm{mo}_{2}, P_{t 2}$

Far downstream

symbols: $V_{3}, P_{3}, M_{3}, \mathrm{mo}_{3}, A_{3}, P_{t 3}$

NOTES

The speed of sound is denoted by the letter ' $c$ ' instead of 'a' as defined in the thesis.

SPECIFICATIONS

Specify $P_{\text {inout }}, \operatorname{mo}, M_{z}$ and $A_{\text {disk }}$ of the freestream

$M_{0}=0.8$

$P_{0}=14.7 \cdot 144 \quad\left[\mathrm{lbf} / \mathrm{ft}^{2}\right]$

$\rho_{0}=0.002329 \quad\left[\right.$ slug/ft $\left.{ }^{3}\right]$

Adlsk $=1\left[\mathrm{ft}^{2}\right]$

$\gamma=1.4$

Velocity Ratio

$r=\frac{V_{3}}{V_{0}}$

$r=0.557$

Assumptions

$P_{0}=P_{3}$

$\rho^{0}=\rho^{3}$

$\mathrm{A}_{3}=\mathrm{Adllsk}_{\mathrm{k}}$

Short hands 
File:Compressible Ducted Turbine Final.EES

4/28/2017 1:45:44 PM Page 2

EES Ver. 10.096: \#0552: for use only by students and faculty, Mechanical Engineering, Dept. Cal Poly State University

$\mathrm{k} 1=\frac{\gamma-1}{2}$

$\mathrm{k} 2=\frac{\gamma}{\gamma-1}$

ORDER OF EQUATION

SOLVING FOR ALL NECESSARY CONDITIONS AT 1

Far upstream conditions at 0

$c_{0}=\sqrt{\gamma \cdot \frac{P_{0}}{\rho_{0}}}$

$M_{0}=\frac{V_{0}}{c_{0}}$

Solve for $V_{0}$

Far downstream conditions at 3

$c_{3}=\sqrt{7 \cdot \frac{P_{3}}{\rho^{3}}}$

$M_{3}=\frac{V_{3}}{c_{3}}$

Solve for $V_{3}$

Total Drag

$D_{\text {total }}=\dot{m} \cdot\left[V_{0}-V_{3}\right]$

Solve for $D_{\text {total }}$

Pressure Ratio 0-1

$\frac{P_{00}}{P_{0}}=\left[1+k 1 \cdot M_{0}^{2}\right]^{12}$

$\frac{P_{t 1}}{P_{1}}=\left[1+k 1 \cdot M_{1}^{2}\right]^{k 2}$

$P_{10}=P_{t 1}$

Solve for $P_{1}$

Density ratio 0-1 
File:Compressible Ducted Turbine Final.EES

4/28/2017 1:45:44 PM Page 3

EES Ver. 10.096: \#0552: for use only by students and faculty, Mechanical Engineering, Dept. Cal Poly State University

$\frac{\rho_{1}}{\rho_{0}}=\left[\frac{P_{1}}{P_{0}}\right]\left[\frac{1}{\gamma}\right]$

Solve for rho,

Energy equation 0-1

$E_{0}=\frac{V_{0}^{2}}{2}+k 2 \cdot \frac{P_{0}}{\rho_{0}}$

$E_{1}=\frac{V_{1}{ }^{2}}{2}+k 2 \cdot \frac{P_{1}}{\rho 1}$

$E_{0}=E_{1}$

Solve for $V_{1}$

Velocity ratio 0-1

$\frac{V_{1}}{V_{0}}=\frac{\rho_{0}}{\rho_{1}} \cdot \frac{A_{0}}{A_{\text {dlsk }}}$

Solve for $A_{\text {disk }}$

SOLVING FOR ALL NECESSARY CONDITIONS AT 2

Area Ratio 0-3

$\frac{A_{3}}{A_{0}}=\frac{\rho_{0}}{\rho^{3}} \cdot \frac{V_{0}}{V_{3}}$

Solve for $\mathrm{A}_{3}$

Area ratio 2-3

$\frac{A_{3}}{A_{d 15 k}}=\frac{M_{2}}{M_{3}} \cdot\left[\left(\frac{1+k 1 \cdot M_{3}{ }^{2}}{1+k 1 \cdot M_{2}{ }^{2}}\right)^{\left(\frac{?+1}{4 \cdot k 1}\right)}\right]$

Solve for $\mathrm{M}_{2}$

Pressure ratio 2-3

$\frac{P_{\mathrm{t} 2}}{\mathrm{P}_{2}}=\left[1+\mathrm{k} 1 \cdot \mathrm{M}_{2}{ }^{2}\right]^{\mathrm{K} 2}$ 
File:Compressible Ducted Turbine Final.EES

4/28/2017 1:45:45 PM Page 4

EES Ver. 10.096: \#0552: for use only by students and faculty, Mechanical Engineering, Dept. Cal Poly State University

$$
\begin{aligned}
& \frac{P_{t 3}}{P_{3}}=\left[1+k 1 \cdot M_{3}^{2}\right]^{K 2} \\
& P_{t 2}=P_{t 3}
\end{aligned}
$$

Solve for $P_{2}$

Density ratio 2-3

$\frac{\rho^{2}}{\rho^{3}}=\left[\frac{P_{2}}{P_{0}}\right]\left[\frac{1}{\gamma}\right]$

Solve for $\mathrm{rhO}_{2}$

Energy equation

$E_{2}=\frac{V_{2}{ }^{2}}{2}+k 2 \cdot \frac{P_{2}}{\rho^{2}}$

$E_{3}=\frac{V_{3}^{2}}{2}+k 2 \cdot \frac{P_{3}}{\rho^{3}}$

$E_{2}=E_{3}$

Solve for $\mathrm{V}_{2}$

Turbine Drag

NEED $V_{1}, V_{2}, P_{1}, P_{2}$

$D_{\text {turo }}=\dot{m} \cdot\left[V_{1}-V_{2}\right]+A_{d l s k} \cdot\left[P_{1}-P_{2}\right]$

$T_{\text {lip }}$

$T_{11 p p}=D_{\text {turb }}-D_{\text {total }}$

Coefficients

$C_{D, \text { total }}=-1 \cdot \frac{D_{\text {total }}}{\rho_{0} \cdot V_{0}{ }^{2} \cdot \frac{A_{d l s k}}{2}}$

$\mathrm{C}_{T, \| \mathrm{p}}=\frac{\mathrm{T}_{\| \mathrm{p}}}{\rho_{0} \cdot \mathrm{V}_{0}{ }^{2} \cdot \frac{\mathrm{A}_{\mathrm{dlsk}}}{2}}$

$C_{D, \text { turb }}=-1 \cdot \frac{D_{\text {turb }}}{\rho_{0} \cdot V_{0}{ }^{2} \cdot \frac{A_{\text {dlsk }}}{2}}$ 
File:Compressible Ducted Turbine Final.EES

4/28/2017 1:45:45 PM Page 5

EES Ver. 10.096: \#0552: for use only by students and faculty, Mechanical Engineering, Dept. Cal Poly State University

\section{Power equations}

Power available within free-flowing wind in area equal to rotor area $A_{\text {disk }}$

Pavallable $=\rho_{0} \cdot V_{0}{ }^{3} \cdot \frac{A_{d l s k}}{2}$

Power production at turbine

$\frac{P_{\text {extracted }}}{\dot{m}}=\frac{V_{0}{ }^{2}-V_{3}{ }^{2}}{2}$

Power extraction efficiency

$\eta=\frac{P_{\text {extracted }}}{P_{\text {avallable }}}$

Mass Flowrate

$\dot{m}=V_{1} \cdot \rho 1 \cdot A_{d l s k}$

NORMALIZATIONS

Ratios

$V_{10}=\frac{V_{1}}{V_{0}}$

$V_{20}=\frac{V_{2}}{V_{0}}$

$V_{30}=\frac{V_{3}}{V_{0}}$

$M_{10}=\frac{M_{1}}{M_{0}}$

$M_{20}=\frac{M_{2}}{M_{0}}$

$M_{30}=\frac{M_{3}}{M_{0}}$

$P_{10}=\frac{P_{1}}{P_{0}}$

$P_{20}=\frac{P_{2}}{P_{0}}$ 
File:Compressible Ducted Turbine Final.EES

4/28/2017 1:45:45 PM Page 6

EES Ver. 10.096: \#0552: for use only by students and faculty, Mechanical Engineering, Dept. Cal Poly State University

$\rho 10=\frac{\rho 1}{\rho 0}$

$\rho_{20}=\frac{\rho^{2}}{\rho_{0}}$

$A_{01}=\frac{A_{0}}{A_{d 15 k}}$

$A_{31}=\frac{A_{3}}{A_{d l s k}}$

Pressure-rise Coefficient

$C_{6, P}=\frac{P_{2}-P_{1}}{0.5 \cdot \rho_{0} \cdot V_{0}{ }^{2}}$

Dimensionless mass flowrate

$\dot{\mathrm{m}}_{d}=\frac{\dot{\mathrm{m}}}{\rho_{0} \cdot \mathrm{V}_{0} \cdot A_{\mathrm{d} l \mathrm{sk}}}$ 